\title{
SOLAR ENERGY SYSTEM INSTALLED AT THE NORTH GEORGIA APDC OFFICE BUILDING
}

\section{Prepared by}

North Georgia Area Planning and Development Commission 503 West Waugh Street

Dalton, Georgia 30720

Under DOE Contract No. EG-77-A-01-4075

Monitored by

National Aeronautics and Space Administration

George C. Marshall Space Flight Center, Alabama 35812

For the U. S. Department of Energy

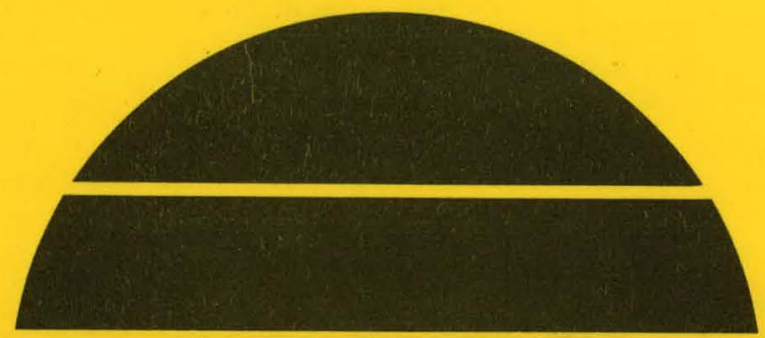

\section{U.S. Department of Energy}




\section{DISCLAIMER}

This report was prepared as an account of work sponsored by an agency of the United States Government. Neither the United States Government nor any agency Thereof, nor any of their employees, makes any warranty, express or implied, or assumes any legal liability or responsibility for the accuracy, completeness, or usefulness of any information, apparatus, product, or process disclosed, or represents that its use would not infringe privately owned rights. Reference herein to any specific commercial product, process, or service by trade name, trademark, manufacturer, or otherwise does not necessarily constitute or imply its endorsement, recommendation, or favoring by the United States Government or any agency thereof. The views and opinions of authors expressed herein do not necessarily state or reflect those of the United States Government or any agency thereof. 


\section{DISCLAIMER}

Portions of this document may be illegible in electronic image products. Images are produced from the best available original document. 
This report was prepared to document work sponsored by the United States Government. Neither the United States nor 1ts agents the United States Department of Energy, the United States National Aeronautics and Space Administration, nor any federal employees, nor any of their contractors, subcontractors or thelr employees, make any warranty, express or 1mplied, or assume any legal liability or responsibllity for the accuracy, completeness, or usefulness of any information, apparatus, product or process disclosed, or represent that its use would not infinge privately owned rights. 


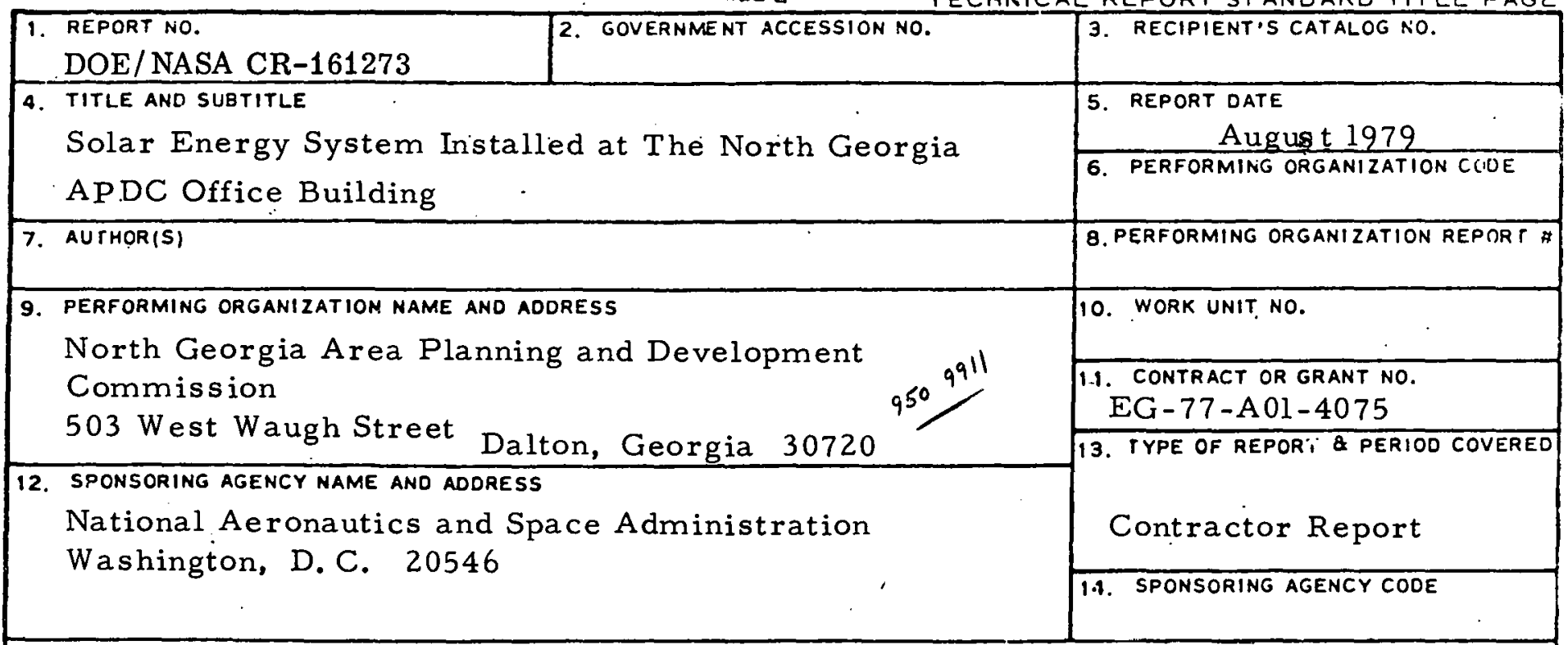

15. SUPPLEMENTARY NOtes The project manager for this work was $\mathrm{Mr}$. D. W. Kolberg of the North Georgia Area Planning and Development Commission (APDC). This work was done under the technical management of Mr. Douglas W. Westrope Jr. George C. Marshall Space Flight Center. Alabama.

16. ABSTRACT

This final report provides information on the solar energy system installed in the newly constructed office building of the North Georgia Area Planning and Development Commission near downtown Dalton, Georgia. This sytem was partially funded by the Department of Energy under contract EG-77-A-01-4075.

This solar heating, cooling and hot water system supplies 65 to $70 \%$ of the cooling demand and 90 to $95 \%$ of the heating demand. There are 2, 001 square feet of effective Revere collector area, and the absorption chiller is an Arkla model 300 and provides 16 tons of cooling. 
THIS PAGE

\section{WAS INTENTIONALLY LEFT BLANK}




\section{Table of Contentyd}

Part

Page Number

1. Description of the North Georgia Area Planning and Development Commission Solar Energy
System and Building

2. Acceptance Test Data

Test plan

Test Results

$2-2$

$2-6$

3. As-Built Drawings

1) Simplified Hydronic Schematic

2) Detailed Hydronic Schematic

3) Solar Control Wiring Diagram

4) Wiring Diagram

$3-3$

$3-4$

$3-5$

4. Operation and Maintenance

5. Photos of System and Building

6. Predicted System Performance

7. Problems

8. Recommendations

9. Verification Statements

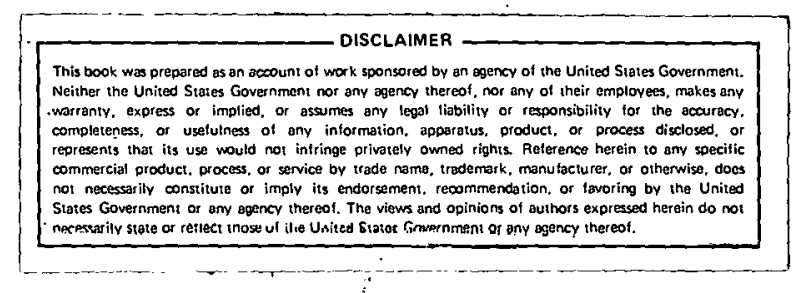

iii 


\section{PART I}

\section{DESCRIPTION OF THE SOLAR ENERGY SYSTEM}

AND BUILDING

\section{Introduction}

The North Georgia Area Planning and Development Commission (APDC) office building is equipped with a hydronic, automatic drain-down solar heating and cooling system. The system, providing solar heat exchange from a 2,001 square foot effective collector area, supplies 65-70\% of the building's cooling demand and $90-95 \%$ of the heating demand. Domestic hot water is also provided, however, the demand is very nominal. Heating is accomplished by the direct flow of solar heated water to an exchange deck in the central air handler. The building is cooled by the direct flow of chilled water to a separate exchange deck. In the cooling mode, solar heated water is used to drive a 25 ton absorption chiller. Back-up or supplementary supply of heated water to drive the chiller or for building heat is provided by a conventional oil-fired boiler. Control sequencing for heating and cooling modes is accomplished by a multizone central processor. Day and night demands for heating and cooling are controlled by a standard automatic time clock and an override, low setting thermostat.

\section{North Georgia APDC Office Building}

The newly constructed office building, located on 1.5 acre lot near downtown Dalton, Georgia, is a modern, two-story structure containing 7,000 square feet of office and work space. The building has masonry exterior walls, steel frame floor and roof system, and type 4 fire-resistive construction. 
The building has numerous energy conservation features incorporated into its construction. The western and northwestern, first floor elevations of the building are buried into the sloping contour of the lot reducing the summertime afternoon sun load and minimizing the cooling effect of the predominately northwestern winter wind. The square shape of the building produces the least amount of exterior wall area which reduces the heating and cooling load. Also, the two-story design of the building has minimized the roof area further reducing the loads on the building. Window area has been kcpt to a minimum. There are no windows in the west wall thus eliminating a potential source of heating load from the afternoon sun. Windows have been confined to the east wall and adjoining corners and are protected from the sun by generous roof overhang. All windows are operable so that under appropriate weather conditions natural ventilation is available. Window glass is the insulating type, double glazed, and shaded to prevent the impingement of direct sunlight.

The respective $U$ factors for the roof and walls are .09 and .04 .

\section{Collector Array}

The fiflh, or southern most row of collectors, is not reflector augmented but is pitched at an angle of 25 degrees. The reason for the different configuration of this row is due to the limitation of roof area to accommodate the fifth collector. The design indicated that a fifth row would significantly enhance the summertime delivery of energy (the period of highest energy demand). Since the roof area was too small for the placement of both collectors and reflectors in this row, only the collector bank was used and was placed at an angle to absorb the maximum summertime energy. 
Collectors are double glazed with special low iron glass and have an application of black chrome selective coating. Reflector panels were fabricated from stainless steel sheets (gage .02 inches) mounted with adhesive to $5 / 8$ inch marine plywood.

The collector array support structure is fabricated from welded steel into a truss design and mounted to the roof on specially constructed roof curbs. The truss design, in addition to providing considerable strength against possible wind damage, facilitated the installation of the collectors and reflector panels. As each collector and panel, including their support frame was placed in position on the array structure, each was rotated from the vertical into the structure at the appropriate angle and welded into place. The steel support structure was coated with a corrosion resistant paint.

\section{Absorption Chiller}

Solar cooling of the North Georgia APDC building is accomplished with a model 300 Arkla absorption chiller. Water heated by solar or the oil-fired boiler is pumped to the chiller at $180^{\circ} \mathrm{F}$ at $54 \mathrm{GPM}$. to provide 16 tons of cooling. At this temperature and flow rate, the chiller produces chilled water at $45-50^{\circ} \mathrm{F}$ for circulation through the multizone air handling unit.

\section{Supplementary Energy Source}

Supplementary heating for hot water to operate the absorption chiller or heated water to the thermal deck in the air handling unit is provided by a Weil-Mclain oil-fired boiler, Model 576-EH. Number 2 fuel oil, drawn from a buried, 1,500 gallon storage tank is consumed at 2.95 gallons per hour. 
Space Conditioning Equipment

The North Georgia APDC solar system is interfaced with a conventional seven zone multizone air handler unit (AHU) with ducted air distribution to each zone. Each zone has an independent thermostat for effecting desires set point conditions for the air which is ducted to each zone. This air is either heated, cooled, or recirculated through the multizone unit.

Two tube-in-fin heat exchanges are employed in the AHU. Hot and chilled water is circulated through their respective coils according to the demand of the seven-zone thermostats. The AHU's circulating fan operates continuousily during the day when the building is occupied to provide air circulation and humidity control. An override setback switch is used to operate the system during off-duty hours. After the first cooling and heating seasons, the system was modified to prevent the simultaneous flow of hot and chilled water to the AHU. This was accomplished by installing manual selection switches for hot or chilled "only". switches.

System Controls and Modes of Operation 1

The system employs a Multizone Central Processor (MCP) for establishing appropriate control sequencing depending on the heating and cooling demand placed on the system. Solar collection controls operate separately but in conjunction with the MCP.

Solar Collection - Pump.P-1 circulates water through the solar collectors and is controlled through switching relay $\mathrm{R}-1$ and motor starter relay $\mathrm{C}-1$. Switching relay $R-1$ is controlled by a temperature difference sensing device with one sensor (TS-1) on the absorber plate of a particular collector. The other sensor (TS-2) is located in the bottom of the hot water storage tank 
(HWST). When TS- 1 minus TS-2 reaches a level $15^{\circ} \mathrm{F}$, relay $\mathrm{R}-1$ closes, activating motor starter $\mathrm{C}-1$. $\mathrm{P}-1$ begins to fill the array and circulate water through the collectors. If TS-1 minus TS-2 drops to a level of $5^{\circ} \mathrm{F}, \mathrm{R}-1$ reopens and P-1 ceases operation allowing the water in the collectors to drain back into the HWST.

The only exception to this operation is when the chiller is being powered with solar heated hot water. In this case $R-1$ is overridden by $R-6$, hence $P-1$ is "locked on" until R-6 returns to its N.0. position and R- 1 is again the sole controller of P-1. R-6 overrides R-1 because the position of R-1 is related to the temperature of the hot water tank which is not in the "system" during the AC mode.

Heating - Solar and Boiler - Heat is delivered to the building by a finned tube heat exchanger. Hot water is passed through the tubes and air is blown through the fins and then distributed to the appropriate areas by the multizone air handling unit. Heat for the building is called for in two stages (min. and max.) depending on how much heat is required to bring temperature back up to its set point. Minimum and maximum refers to the difference between the thermostat set point and the sensed room temperature which sets a priority on energy sources. Due to the nature of the building, it is possible to require heat in one area of the building and cooling in another. To save energy at the cost of slight discomfort, an isolation relay $R_{18}$ has been installed so that when the chiller is operating, no heating equipment can be operated. Heat can be supplied through modulating valve $V-6$. (See page 1-9)

If min. heat is called for, $R_{20}$ closes, assuming the chiller is off, the top of the HWST is checked to see if the temperature there (TS-3) is over $120^{\circ} \mathrm{F}$ $\left(49^{\circ} \mathrm{C}\right)$. If it is, C-2 (motor starter) closes causing P-2 to circulate hot water through the heating coil. If TS-3 senses a temperature below $120^{\circ} \mathrm{F}\left(49^{\circ} \mathrm{C}\right)$, no 
heat is delivered. If and when max. heat is called for $R_{9 B}$ closes, if $P-2$ is operating nothing further occurs. When max. heat is called for and P-2 and the chiller are off, the boiler and boiler circulator are turned on by $\mathrm{R}_{19}$ closing.

Air Conditioning - Solar and Boiler - Cooling is delivered to the building by a finned tube heat exchanger, as chilled water is pumped through the tubes and air circulates through the fins. The cooled air is distributed to the appropriate areas by the multizone air handling unit. On the solar collector array, a solar intensity sensor is lncated in a position to measure the combined reflected, beam and diffuse radiation falling in the plane of the collector panels. When this reaches a preset amount, I on (approximately $160 \mathrm{BTU} / \mathrm{FT}^{2} / \mathrm{HR}$ ), $R_{3}$ closes and activates time delay relay $R_{4} . R_{4}$ holds $R_{3}$ closed for three (3) minutes to prevent any rapid cycling. $R_{3}$ will open when the combined solar radiation drops to a level $\mathrm{I}_{\text {off }}$ (approximately $140 \mathrm{BTU}^{\mathrm{FT}}{ }^{2} / \mathrm{HR}$ ), provided the three minute time delay has reopened. If the outside air temperature is above a preset limit $T_{O A}{ }^{l}\left(65^{\circ} \mathrm{F}\right), R_{5}$ closes. If $R_{5}$ is closed and $R_{3}$ is closed, the following occurs simultaneously: $R_{6}$ locks on $P-1, R_{7}$ closes preparing to operate the chiller, $R_{R}$ operates $V_{1}$ and $V_{2}$ which begin to preheat the water in the piping, rollectors; and buffer tank to operate the chiller. $R_{12}$ closes, preventing the boiler from operating. When the outlet of the buffer tank reaches $165^{\circ} \mathrm{F}\left(74^{\circ} \mathrm{C}\right), \mathrm{R}_{10}$ closes and the chiller begins operating. The chiller's own internal controls operate diverting valve $V-3$.

All of the above occurs without regard to the cooling load of the building. If cooling is called for, mixing valve $V-5$ begins to deliver chilled water to the cooling coil in the air handler in the solar A.C. mode.

Cooling is called for in two stages min. and max. depending on how far the senses room air temperature is above the set point. If the chiller is not 
operating from solar and min. cool is called for, $\mathrm{R}_{13}$ closes operating motor starter C-6 which operates P-6 the chilled water circulator. If the senses room temperature continues to rise sufficiently to call for max. cool, the boiler and boiler circulator and the chiller are all brought into full operation. As min. cool is always on when max. cool is, the chilled water circulator pump is still operating.

On weekends the building is not cooled and any solar chilled water is put into storage. When the bottom of the chilled water storage tank, (TS-4) reached $55^{\circ} \mathrm{F}\left(12.8^{\circ} \mathrm{C}\right)$, the boiler cannot operate for a maximum cooling demand as it would during the working week days.

Domestic Hot Water Heating - Domestic hot water is heated in the domestic hot water tank by either solar hot water or electrical resistance heat. Electrical heat is only used when the top of the tank is cold. P-7 circulates water from the top of the HWST through a heat exchanger in the domestic hot water tank. and returns it to the bottom of the HWST. This pump is controlled through $R_{22}$, a differential temperature sensing device. This device compares the temperature (TS-5) at the top of the HWST and the temperature (TS-6) at the bottom of the domestic hot water tank. When TS-5 minus TS- 6 reaches a value of $T_{\text {on }}\left(15^{\circ} \mathrm{F}\right)$, $R_{22}$ closes and $P-7$ begins operation. When TS -5 minus TS- 6 drops to a value of $T_{\text {off }}\left(5^{\circ} \mathrm{F}\right), R_{22}$ opens and $P-7$ ceases operation. 


\section{RELAY LEGEND}

$R_{1} \quad$ collector $\Delta T$

$R_{2 B} \quad \max$. c00l

$R_{3}$. . solar cell

$\mathrm{R}_{4}$ time delay

$R_{5} \quad 0 . A$. temp.

$R_{6}$ solar $A C$

$R_{7}$ solar $A C$

$R_{8}$. solar $A C$

$R_{\cap R}$ max. heat

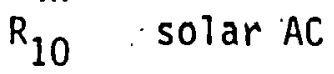

$\mathrm{R}_{11}$ solar $A C$

$R_{12}$ boiler lockout:

$R_{13}$. min. cool

$R_{14}$. C.W. storage tank

$R_{15}$ C.W. circulator

$\mathrm{R}_{16}$ boiler start

$\mathrm{R}_{17}$ boiler $\mathrm{AC}$

$\mathrm{R}_{18}$

$\mathrm{R}_{19}$

$\mathrm{R}_{20}$

$\mathrm{R}_{21 \mathrm{~A}}$

$\mathrm{R}_{21 \mathrm{~B}}$

$\mathrm{R}_{22}$

$c_{1} \quad \mathrm{P}=1$

$\mathrm{C}_{2} \quad \mathrm{P}-2$

$\mathrm{C}_{3} \quad \mathrm{P}-3$

$C_{4} \cdot P-1$

$C_{5} \quad P-5$

$C_{6} \quad P-6$

$C_{7} \quad F-1$
NO operates collector circulator pumps

NO : boiler A.C., "SE2TV\#1

NO close sufficient insolation. for solar $A C$

NO locks on $R_{3}$ at first turn on

NO for solar AC, RDK\#1

NO override $R_{1}$

NO preheat fluid for solar AC

NO operates $V_{1}$ and $V_{2}$

NO boiler heat

NO buffer outlet sufficient for solar AC RDK\#2

NO operates chiller for solar AC

NC will open circuit boiler circuit

NO operate C.W. circulating pump SE2TV\#1

NC no boiler AC if open RDK\#3

NO operate $C . W$. circulator when boiler $A C$

No operate boiler on max. $A C$ if no solar $A C$

NO, operates chiller for boiler $A C$

NC locks out heating equip. if chiller is on

NO boiler on for boiler AC or boiler heat

NO "operate solar H.W. circulator

NO allows boiler heat if no heat in storage

NO allow heating from solar H.W.

NO operates DHW circulator pump

No collector circulator pump

NO hot water circulator pump

NO boiler circulator pump

No condensing water circulating pump

NO evaporator circulator pump

NO chilled water circulator pump

- NO cooling tower fan 


\section{Modifications}

The North Georgia APDC solar system has undergone two modifications during the operational period May through December 1978. In both cases these modifications were changes from the original system design.

Under the original design specifications the system could operate simultaneously in both the heating and cooling modes. This was not a problem during the summer period when the system was operating under a maximum cooling load. However, during the fall, the building would require heating in the morning hours and cooling in the afternoon. Another noticeable situation during this period was the in balance between the first and second floors. Often the first floor would call for heating while the second floor would call for cooling. The overall situation created an ineffective, intermittent operation of the chiller. The controls were modified to include manual switches to keep the system in one specific mode.

The second modification resulted from the freezing of the collectors. To insure adequate, full drainage of the collectors, vacuum breakers have been installed on the supply headers on each row of collectors. (These can be seen in Photograph \#5.) 
PART 2

ACCEPTANCE TEST DATA

$2-1$ 


\section{ACCEPTANCE TEST PLAN \\ NGAPDC \\ DALTON, GEORGIA}

\section{Demonstrate Fail Safe Controls}

The system will be placed in each mode of operation and the main power disconnect will be interrupted. Response of the overall system will be observed to insure that no unsafe conditions arise, that no equipment is damaged or rendered ntherwise inoperable, and that the collector array drains fully. Power will be applied and the system observed to insure that normal operation resumes without external intervention.

2. Demonstrate Pressure Relief Valves

The mechanical equipment loops with pressure relief valves will be isolated from the thermal storage tanks and their respective loops pressurized to insure that the pressure relief valves operate at their designated pressure.

3. Demonstrate No Leaks

Prior to installation of insulation the overall system will be isolated from the thermal storage tank and pressurized to 15 psig and then isolated from the pressure source. Pressure will be observed for 6 hours to insure no leaks are present.

4. Demonstrate No Growth of Algae, Fungi, Mold or Mildew A similar system has been in operation at the Shenandoah, Georgia, Community Center for one year with no observef foreign growth. The wat.er in Dalton, which is used in the NGAPDC system, has a very low calcium carbonate $\left(\mathrm{CaCO}_{3}\right)$ content, approximately $15 \mathrm{ppm}$.

5. Demonstrate Back-Flow Prevention

The solar hydronic system is connected to the potable water supply in three 
places. One is at the inlet to the domestic hot water heater, back-flow is of no concern here. The second connection is through a gate valve to fill the solar system. This valve will be closed when the system is in operation, however a continuous pressure type back-flow preventer will be put in that line (Watts no.9D or equivalent). The third connection is at the cooling tower; at this connection the water is discharged to atmospheric pressure and the overflow level is below the make up water outlet, therefore back-flow will not occur here. No hard connection occurs between the solar system and the potable water supply. A hose is connected to fill the solar system and two must valves opened. The valves are closed and valves shut when filled. The solar system is not pressurized.

6, Demonstrate No Fluttering Only valves designed for flow control use (such as butterfly, globe, or ball valves) are operated partially open. The system will be observed during initial operation to insure no valve fluttering occurs.

7. Demonstrate Drain and Filling

A closed sight glass will be installed at an appropriate location below the collector array for visual indication of complete drain down.

8. Demonstrate Temperature at Various Points

Thermometer wells will be installed at the critical points indicated on the flow schematic. Temperature will be observed and recorded with mercury in glass thermometers.

9. Demonstrate Collector Flow GPM

A "Flow Setter" will be installed in the main collector loop to determine the flow through the collectors.

10. Demonstrate Collector. Flow Pressure Drop Gauge cocks will be installed in the array supply and return lines for measurement using a differential pressure gauge. 
11. Demonstrate System Operates in All Modes of Operation

Manual control overrides will be provided in the control system so that the system can be placed in each mode of operation and observed for proper operation.

12. Demonstrate Pump and Fan Ampere Loads

The power wiring will be installed so that each branch circuit is accessible to measurement by an inductive ammeter. Current will be observed to insure operation at rated amperage and that conductor capacity is not exceeded.

13. Verify Heat Collection Performance

The flow rate through the array is measured as previously discussed. The temperature rise of collector water across the array will be measured with a differential thermopile. Insolation on the collector aperture will be measured by a pyranometer (Eppley model 2 or equivalent) mounted at the inclination of the collectors. Output from the differential thermopile and the pyranometer will be indicated on a strip-chart recorder. Ambient temperature will be measured by a mercury in glass thermometer. Steady state conditions will be obtained and the collector efficiency computed from these measurements for comparison with manufacturers efficiency data.

14. Verify All Equipment, Piping, Controls, etc., are Installed in the Manner Specified

Visual inspection of the installation will be performed and documented (photographs will be made as necessary) before and after installation of insulation or backfilling as applicable.

15. Verify All Pumps, Controls, Dampers, Fan, and Air Distribution Systems Operate as Specified

Fluid distribution will be monitored using appropriate flow measurement 
devices (Be1l and Gossett Circuit-Setters or equivalent). Controls will be observed for operation under simulated conditions. Air distribution will be monitored by standard techniques using direct-reading pitot tube manometer. Air distribution was checked and set by the Mechanical Contractor, Calhoun Mechanical, Inc. 


\section{ATP RESULTS}

1. A11 systems operated normally after power interruption.

2. The boiler pressure relief valve released at 30 PSI.

3. The collector circulator pump had a leak at its seal. The séal was replaced and the leak stopped.

4. No algae, fungi, mold or mildew has been observed in either storage tank. The sump of the cooling tower does show signs of algae growth as expected. The sump is to be drained and cleaned monthity when in use.

5. By virtue of the fact that there is no permanent connection between the potable water supply and solar water, back-flow is no problem.

6. No fluttering has been observed.

7. Filling and draining are visible by a $3^{\prime \prime}$ change in water level of the sight glass.

8. Typical temperature at various points were as follows on the days of the ATP:

Chiller:

$\begin{array}{lcc}\text { generator inlet } & 176^{\circ} \mathrm{F} & \left(80^{\circ} \mathrm{C}\right) \\ \text { generator outlet } & 164^{\circ} \mathrm{F} & \left(73^{\circ} \mathrm{C}\right) \\ \text { condenser inlet } & 83^{\circ} \mathrm{F} & \left(28^{\circ} \mathrm{C}\right) \\ \text { condenser outlet } & 101^{\circ} \mathrm{F} & \left(38^{\circ} \mathrm{C}\right) \\ \text { evaporator inlet } & 59^{\circ} \mathrm{F} & \left(15^{\circ} \mathrm{C}\right) \\ \text { evaporator outlet } & 49^{\circ} \mathrm{F} & \left(9.5^{\circ} \mathrm{C}\right)\end{array}$

Collectors:

inlet

$164^{\circ} \mathrm{F} \quad\left(73^{\circ} \mathrm{C}\right)$

outlet

$176^{\circ} \mathrm{F} \quad\left(80^{\circ} \mathrm{C}\right)$ 
Tanks:

top of hot water storage tank

$162^{\circ} \mathrm{F} \quad\left(72^{\circ} \mathrm{C}\right)$

bottom of hot water storage tank

$151^{\circ} \mathrm{F} \quad\left(66^{\circ} \mathrm{C}\right)$

bottom of chilled water storage tank

$69^{\circ} \mathrm{F} \quad\left(20.5^{\circ} \mathrm{C}\right)$

Coils:

chilled water coil inlet

$50^{\circ} \mathrm{F} . \quad\left(10^{\circ} \mathrm{C}\right)$

chilled water coil outlet

$58^{\circ} \mathrm{F} \quad\left(14.4^{\circ} \mathrm{C}\right)$

hot water coil inlet

N/A

hot water coil outlet

$\mathrm{N} / \mathrm{A}$

9. The collector flow through the 115 collector panels was shown to be 54 GPM $(3.4 \mathrm{~W} / \mathrm{s})$.

10. Collector flow pressure drop at 54 GPM $(3.4 \mathrm{~L} / \mathrm{s})$ was demonstrated to be 4.3 PSI.

11. All systems were demonstrated on manual override and performed as specified.

12. Pump ampere loads demonstrated to be 4.5 and 5.5 amps on 5 and 6 amp rated pumps respectively. The cooling tower drew 4.0 amps. The aforementioned equipment is all 208 VAC 3 phase.

13. The test was performed and data taken for two (2) days of operation. At peak production heat from the collectors generated 204,000 BTU/HR (215 MJ/HR) of chilled water with an input of 284,000 BTU/HR ( $300 \mathrm{MJ} / \mathrm{HR}$ ) of hot water at a chiller inlet temperature of $189^{\circ} \mathrm{F}\left(87^{\circ} \mathrm{C}\right)$ and a roof ambient of $88^{\circ} \mathrm{F}$ $\left(31^{\circ} \mathrm{C}\right)$. The strip chart record of the collector temperature difference and solar radiation arc on record at Independent Living, Inc.

14. Mr. Douglas W. Westrope of the George C. Marshall Space Flight Center acting for DOE witnessed and verified installation as specified.

15. Mr. Douglas W. Westrope witnessed and verified operation as specified. 
PART 3

AS-BUILT DRAWINGS

3-1 


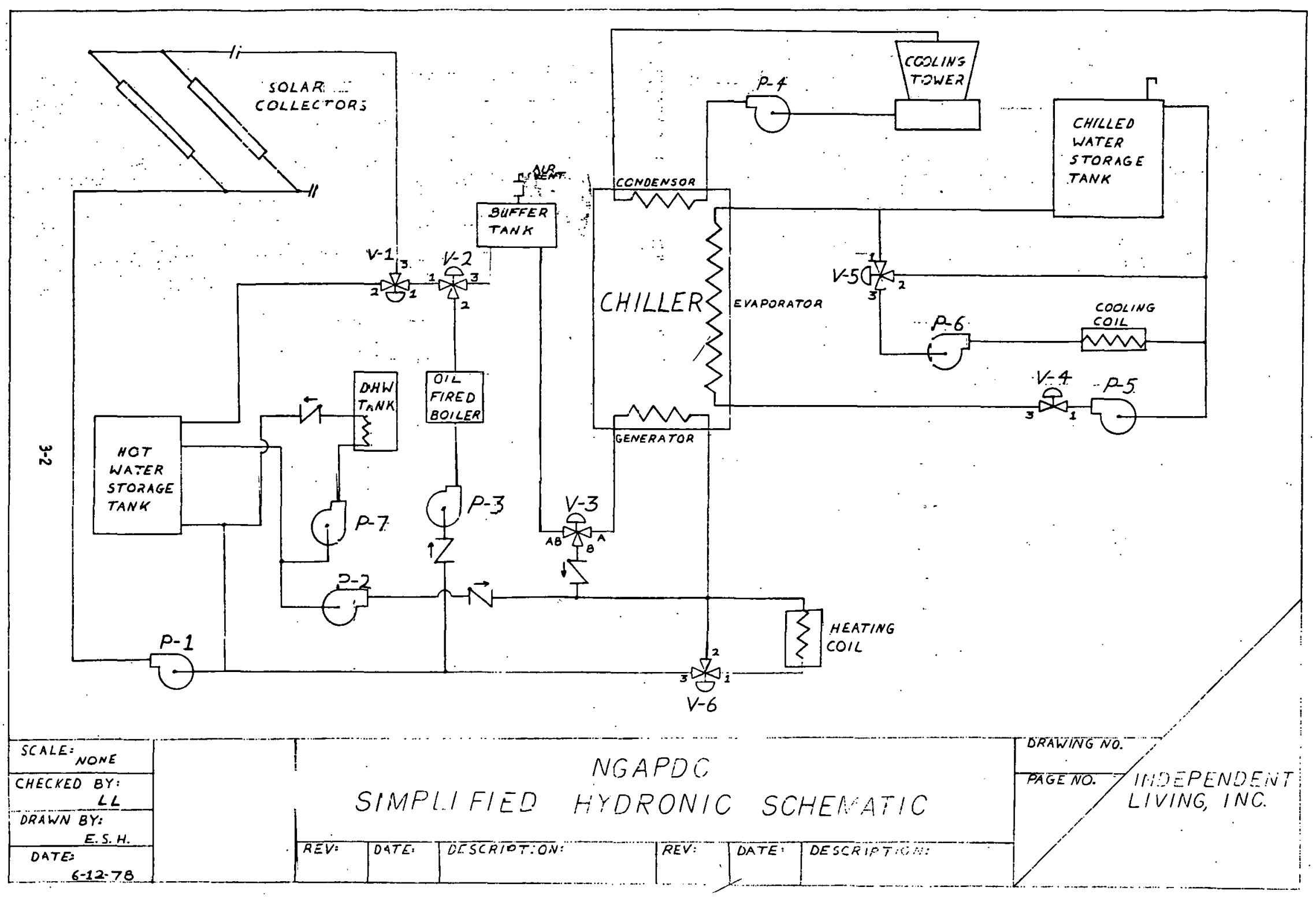




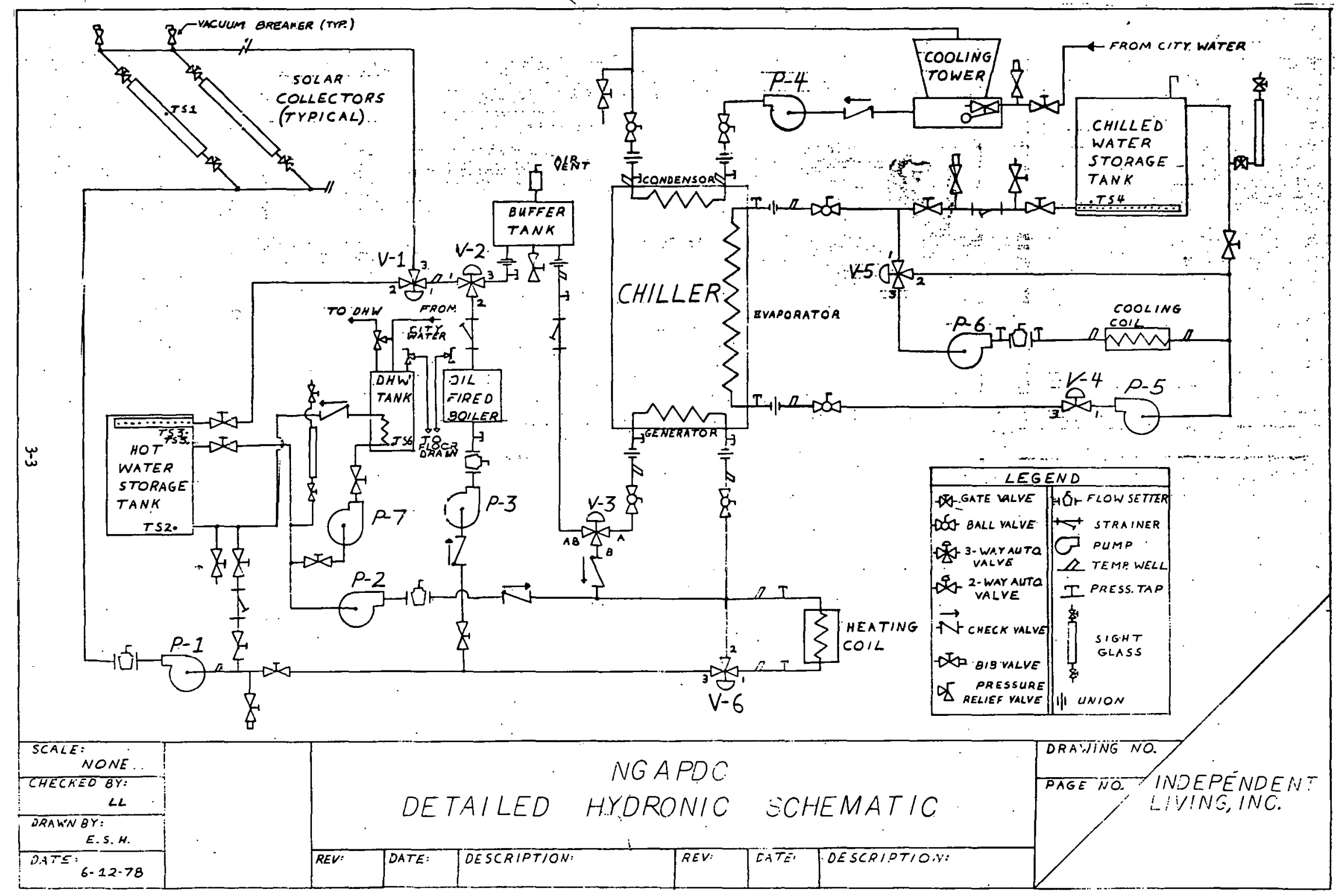




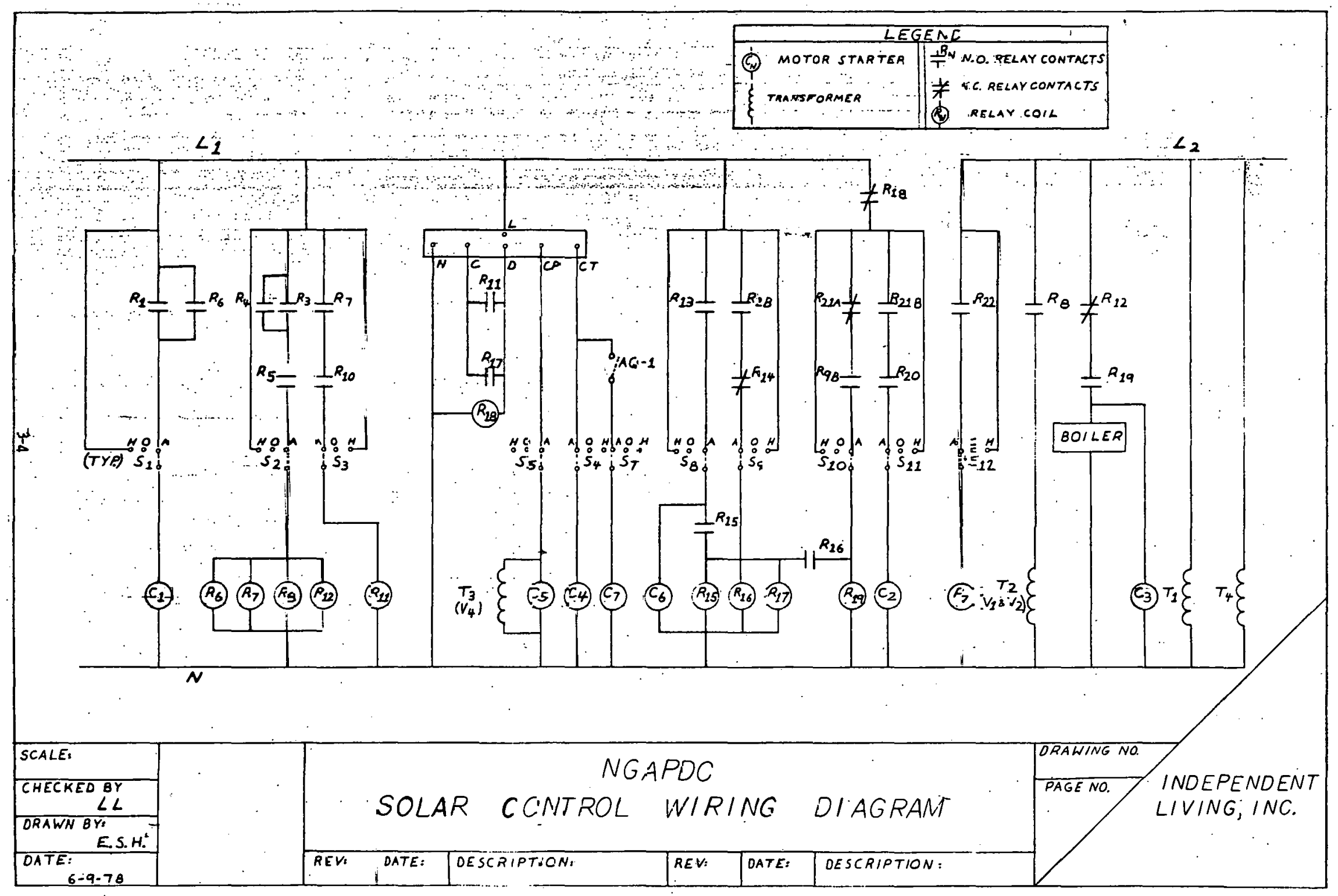




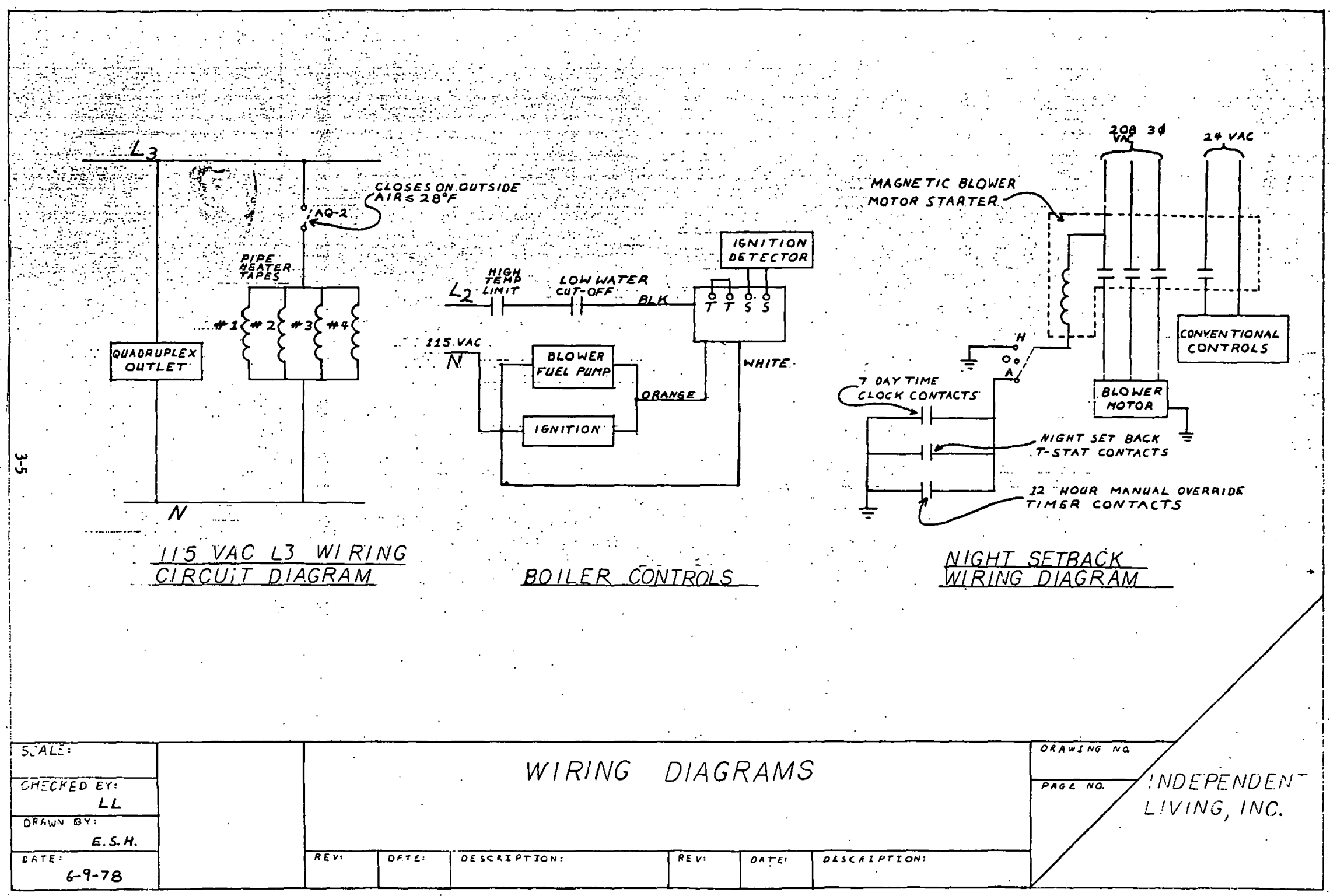


PART 4

SYSTEM OPERATION AND MAINTENANCE

The operation of the North Georgia APDC solar system has been in general, outstanding. The system was activated in late May of 1978 and was tested under the ERDA Acceptance Test Plan on May 24-25. The system has been in continuous operation since that date, and has provided excellent service during the hot, Georgia summer. There have been some problems, but. not of such a nature as to interrupt the overall operation of the system, for any length of time.

As stated previously, the system was designed to produce approximately $60 \%$ of the cooling load. Although the operational record is incomplete, due to reasons which will be explained later, it appears that the system is operating at or exceeding this percentage of the cooling load. One indication that leads to this conclusion is the amount of time required to operate the boiler for supplementary heating of water to operate the chiller. Only an estimate is available at this time, however, the boiler appears to operate for approximately four hours per day during periods of full, unobstructed radiation. The building requires cooling for approximately ten hours each day and the solar system produces a sufficient amount of heated water to drive the chiller for air conditioning for about six hours, iwhich is well within design limits. System performance is expected to improve as more operational experience becomes available.

Over the past nine months the system has provided effective cooling and heating on a continuous basis. The estimate cost of this service has been minimal in comparison to other non-solar cooling systems employed in the local area, and has alleviated the North Georgia APDC of a major operating expense during the summer months. 
Maintenance on the system through February 1979 is as follows:

1. May 24-25, 1978. Seal failure on main circulation pump. Parts under warranty. Labor cost $\$ 100$.

2. June 1, 1978. Solar sensor controls out. of calibration. Materials cost, none. Labor $\$ 160$.

3. June 5, 1978. Seal failure in pump P-1. Replacement high temperature seàl. Materials cost, none. 'Labor $\$ 160$.

4. June 20, 1978. Malfunction of boiler ignitor. Electrodes cleaned and reset. Materials cost, none: Labor cost $\$ 80$.

5. July 10-August 7, 1978. Pump motor (P-1) shorted out. Pump pulled for factory repair. Materials cost, none. Labor cost $\$ 160$.

6. August 15, 1978. Malfunction of control time clock. Clock replaced under warranty. Materials cost, none: Labor $\$ 300$.

7. November 10, 1978... First floor thermostats were initially cross-wired. Materials cost, none.' Labor $\$ 80$.

8. December 8, 1978. Automatic drain down failed causing collector freeze. Piping repair and installation of vacuum breakers. Materials cost, $\$ 370$. Labor $\$ 530$.

9. January 8-25, 1979. Failure of transformer in boiler ignitor system. Transformer, pump, and seals P-2; P-3. Materials cost, $\$ 150$. Labor $\$ 450$.

Note: The above listed maintenance items were covered under a one year warranty. NGAPDC has not incurred any costs for parts or labor. 


\section{MAINTENANCE}

The following contains inforimation pertinent to the solar heating and cooling system installed by Independent Living, Inc. of Atlanta, Georgia on the office building of the NGAPDC in Dalton, Georgia. The system is operational as of May 9, 1978.

The following has three (3) sections. The first contains maintenance instructions and schedules to be performed according to the listed time tables. The second section contains: manufacturers information on the equipment installed in this system. The third contains a description of modes of operation and control sequences. 


\section{WEEKLY CHECK LIST}

1. With system off fill both HWST and CWST with water until flow is observed coming out of vent on top of each tank. This should be done monthly, in the winter only the HWST needs to be filled.

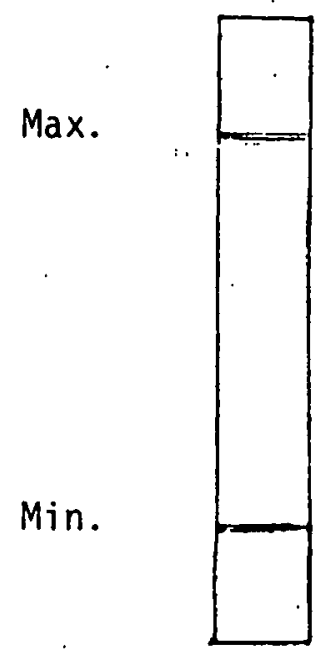

HOT WATER TANK

Water level must be between

the two (2) silver bands.

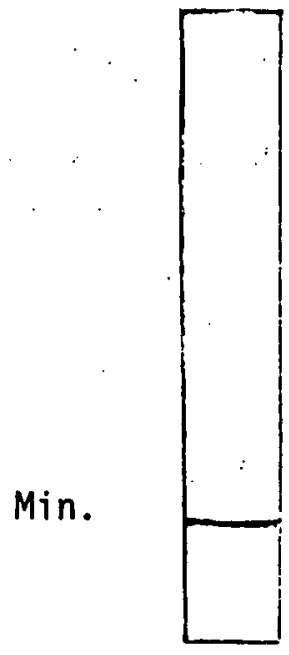

CHILLED WATER TANK

Water level must be above

the silver band.

2. Check to see that circuit breakers are all closed.

3. Observe floor for active leaks (not condensate drip spots!). 
1. Check for correct setting of time clock.

2. Check array for leaks while in collection mode.

3. Check to insure all ten (10) array valves are fully open.

4. Check tempering valve setting $\left(140^{\circ} \mathrm{F}\right)$.

5. Check room thermostat settings for tempering $\left(21^{\circ} \mathrm{F}\right)$.

6. Check blower fan belt.

7. Check blower filters - replace if dirty.

8. Check fuel oil filter for water or dirt, drain if required.

9. Check fuel oil tank level - use a thin nine (9) foot rod.

10. Check storage tanks for vandalism (white seal penetrations).

11. Check $C / T$ fan belt for excess looseness.

12. Check $C / T$ bleed rate while pump is running.

13. Check $C / T$ sump, drain and hose out if required.

14. Check visualiy boiler flue, push 'grey damper for movement.

15. Check control settings a) 0. A. temp $\quad 18^{\circ} \mathrm{C}$

b) Buffer outlet $74^{\circ} \mathrm{C}$

c) C.W. tank temp. $13^{\circ} \mathrm{C}$

d) H.W. tank temp. $50^{\circ} \mathrm{C}$ 


\section{SPRING \& FALL}

1. Lubricate $\mathrm{C} / \mathrm{T}$ fan with $20 \mathrm{~W}$ oil.

2. Lubricate $C / T$ fan motor.

3. Check $C / T$ float valve operation.

4. Drain and hose out $C / T$ sump pan.

5. Lubricate boiler feed pump - fill (2) oil cups.

6. Lubricate circulator pumps.

7. Check heater tape (12 amp o $115 \mathrm{VAC}$ ).

8. Check aquastat on $\mathrm{C} / \mathrm{T}$ - fan on at $85^{\circ} \mathrm{F}$ off at less than $85^{\circ} \mathrm{F}$. 


\section{EACH SPRING}

1. Check pumps and valves for operation.

2. Clean strainers (4).

3. Check dainper actuator operation.

4. Check modes of operation - conventional and solar, record temperature : at critical points.

5. Check boiler linits.

6. Check flow rates.

7. Change fuel oil filter.

8. Replace flow switch on Arkla. (Arkla part no. 14537-169) 


\section{EVERY THREE. (3) YEARS}

1. Replace $C / T$ fan belt.

2. Replace plastic case for fuel oil filter.

3. Repaint rubatex on roof.

4. Touch up any rust spots on array frame. 
PART 5

PHOTOS OF BUILDING AND SOLAR SYSTEM

1. North Georgia APDC Building

2. Collector Array, Storage Tanks

3. Collectors - Reflectors

4. Collector Array Support Structure and Mounts

5. Drain-Down Vacuum Breaker

6. Control Panel

7. Control Module, Pumps, Valves, Buffer Tank

8. Control Module

9. Air Handler showing damper actuators

10. Mechanical Room - Control Module, Chiller, Air Handler, and Boiler 

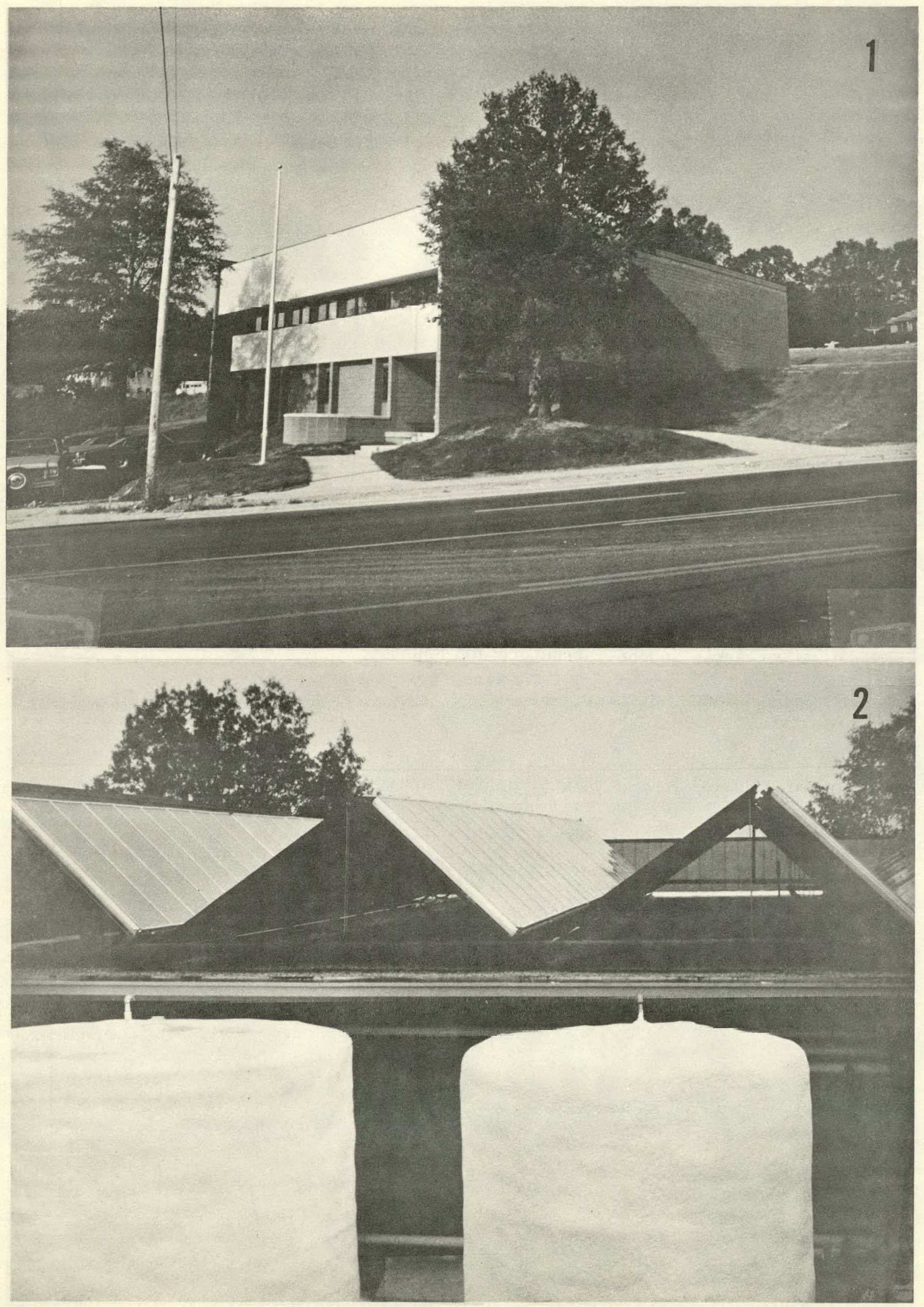

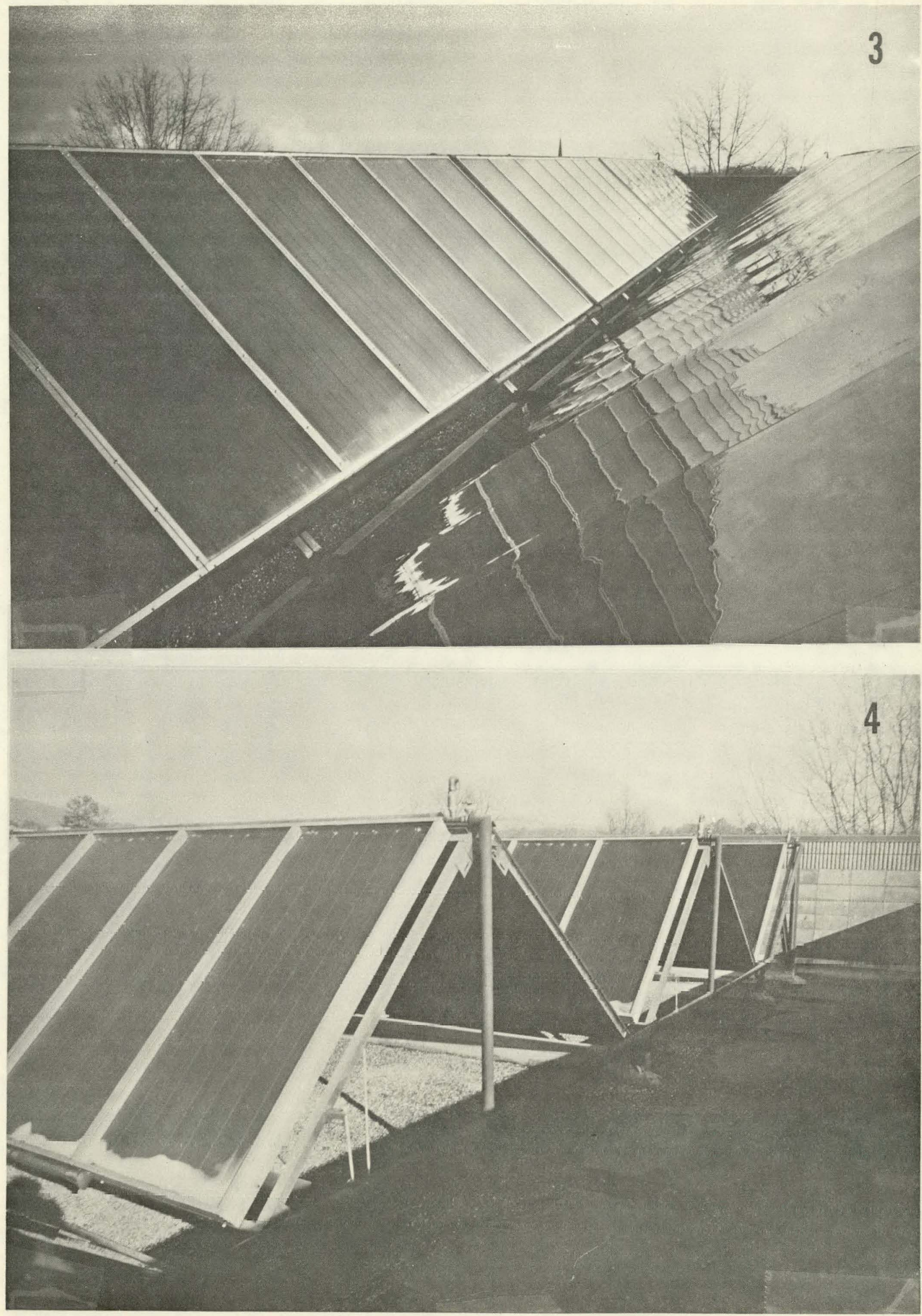

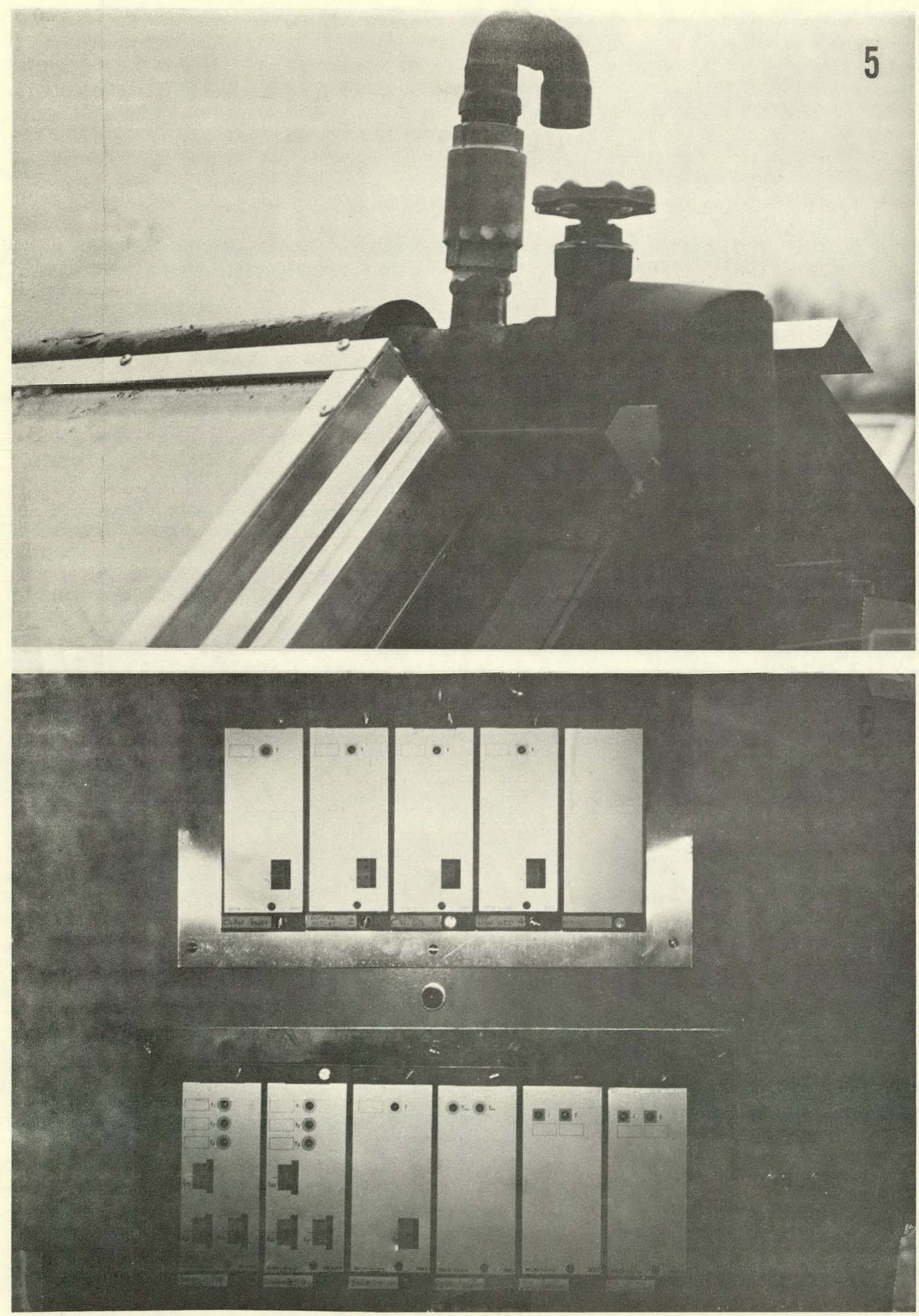


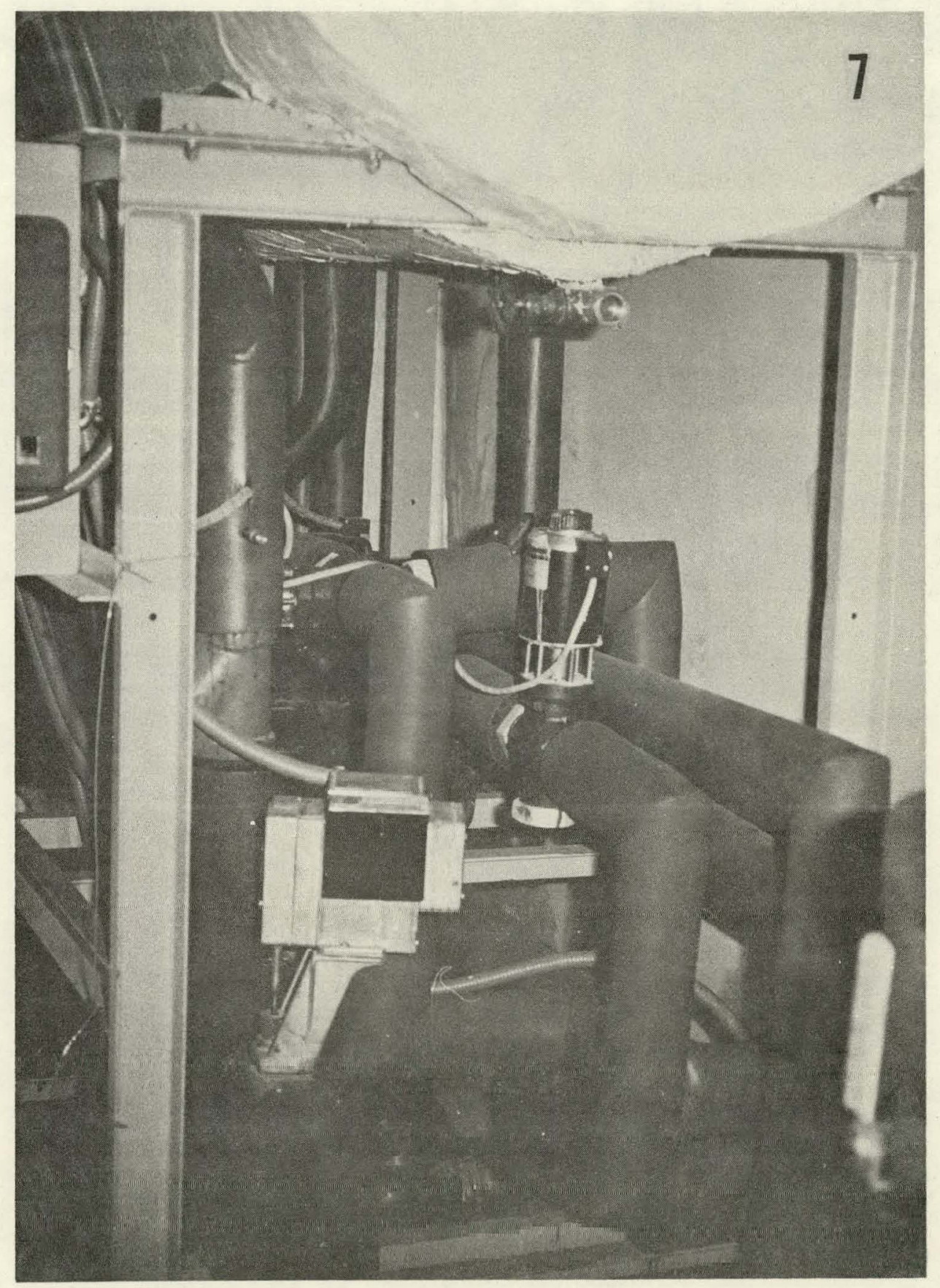




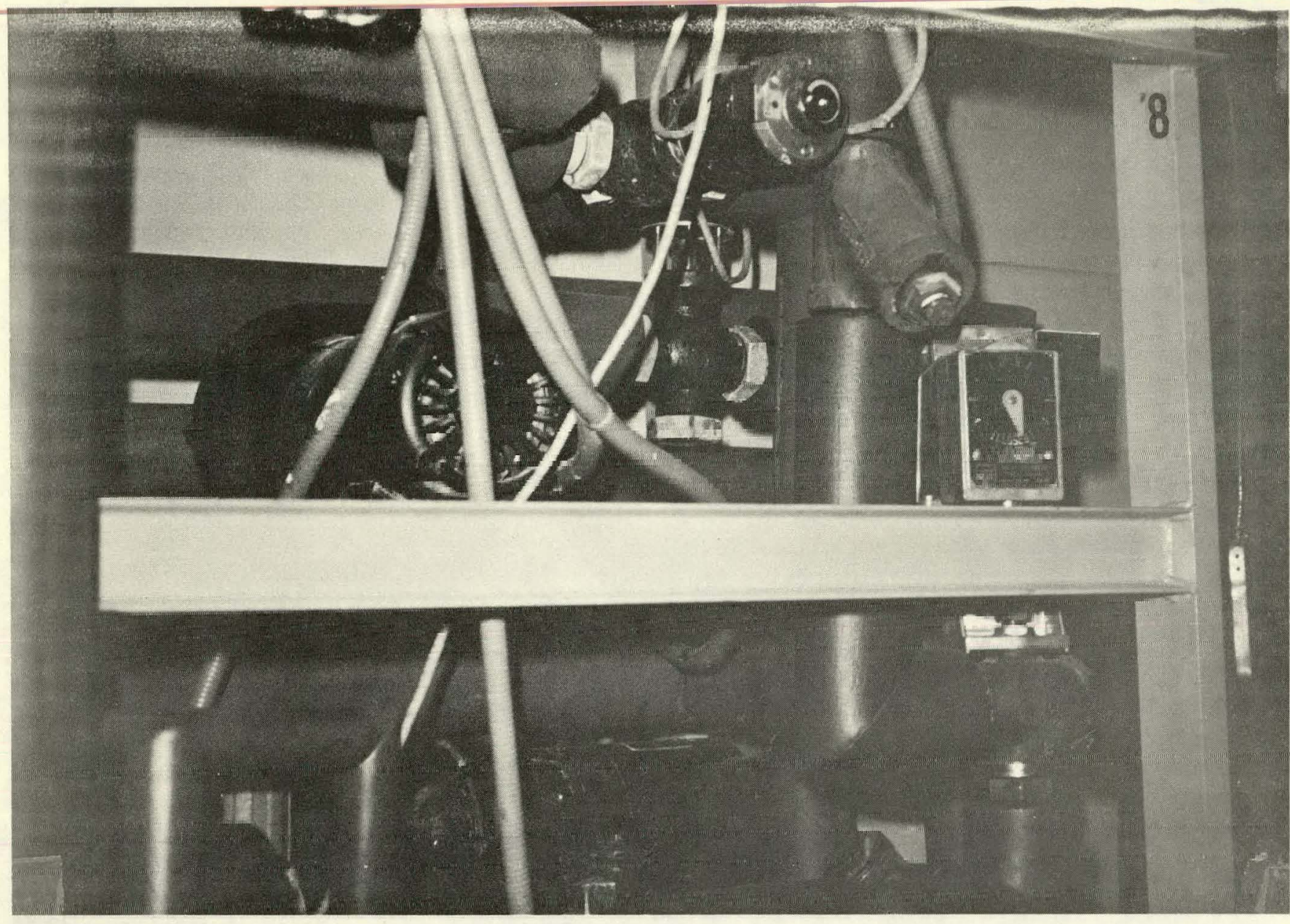

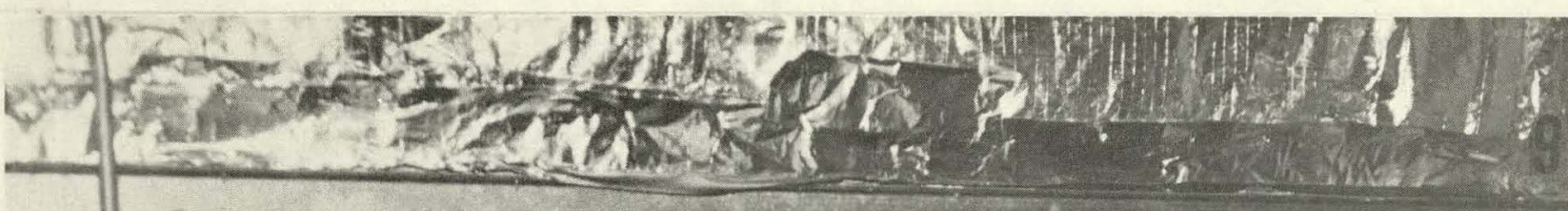
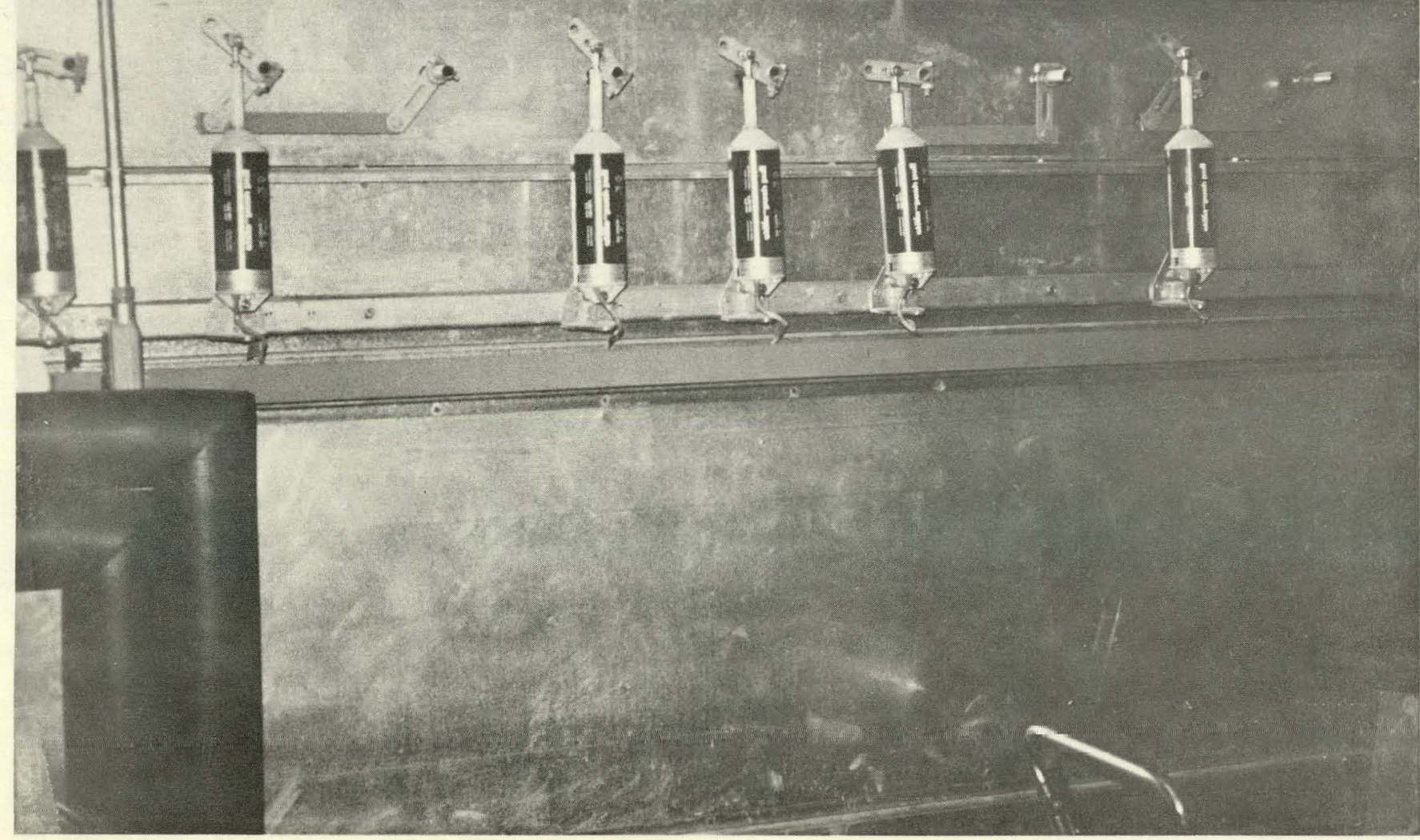


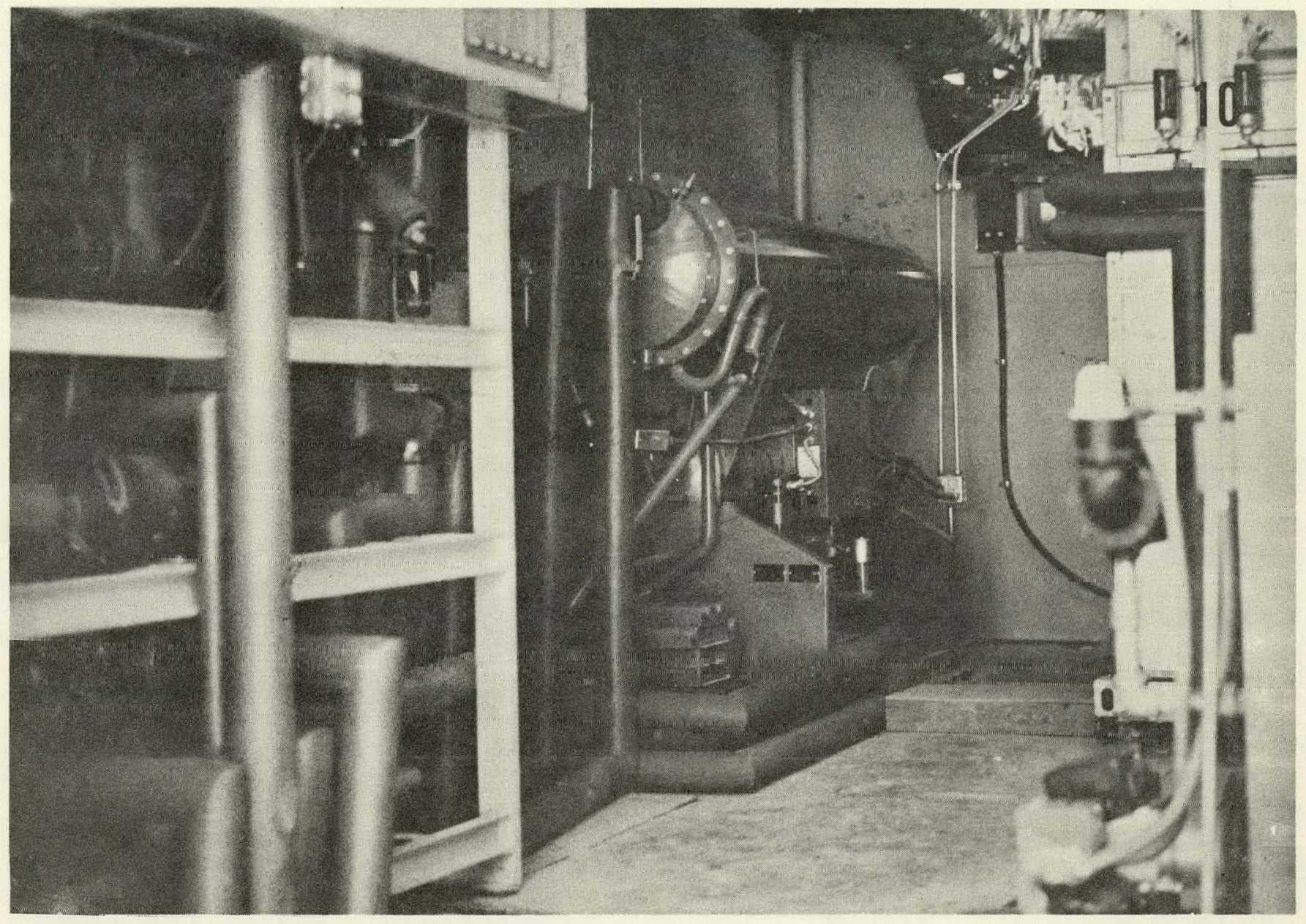


PART 6

PREDICTED SYSTEM PERFORMANCE

SYSTEM AND SUBSYSTEM PERFORMANCE/TECHNICAL DATA

CONTRACTOR SPECIFICATIONS-INDEPENDENT LIVING, INC. 


\section{System and Subsystem Perlormance/Technical Data*}

A. Climatalogical Data:

For the proposed project site provide the following infornation:

1. Latitude 34.5

2. IJeating degree days

\begin{tabular}{l} 
Yearly 3300 \\
January 715 \\
\hline
\end{tabular}

3. Annual Cooling Hours 1700

1. Peak daily insolation 2335 BTU/ft²

5. Yearly sunshine $\quad 58$

I3. Collertor: $\quad$.. Commèrcial/Brand Name

1. Type of Collector.

a. Flate Plate YES

b. Tubular ! N.A.
i) Acceptance Angle
N.A.
ii) Concentration
N.A.
iii) Interception Àrea N.A.
iv) Mirror Reflector Characteristics _ N.A.

c. Concentrator N.A.

i) Focusing N N A.

ii) Non-Focusing N.A.

iii) Tracking $\quad$ N : ; Mode _.N.A.

iv) Non-Tracking N.A. .

v) Concentration Ratio $\frac{\text { N.A. }}{\text { vi) Reflector Reflection }}$

- All data requested in this Appendix must be supplied or a statement given as to why it was omitted. Data requested are :ipecificd for single system or subsystem. If more than one, specify and supply data for each. 
System and Subsyatem Perhormance/Technical Data-Comtinued

2. Transparent Cover

a. Materials

1. Type 1/8" thick tompered glass

2. Composition $\frac{\text { Soda Lime }}{\text { XSG-Clear or }}$

b. Commercial Identification ____ Fourco Clearlite / A.S.G. Water White

c. Solar. Spectrum Transmissivity $\quad 89.58 \quad 91.5 \quad \%$

d. Solar Spectrum Reflectivity _-8 8

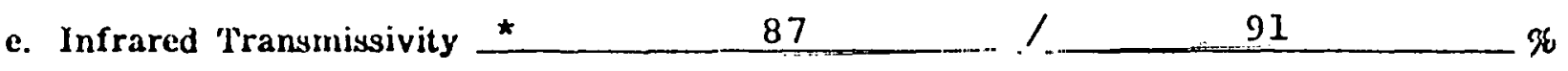

f. Infsisud Rufeutlvity _ـ 8

g. Number of Covers ___ 2

h. Combustibility Noncombustible

i. Edge Treatment _ Swiped - gasket enclosed

j. Physical Properties**

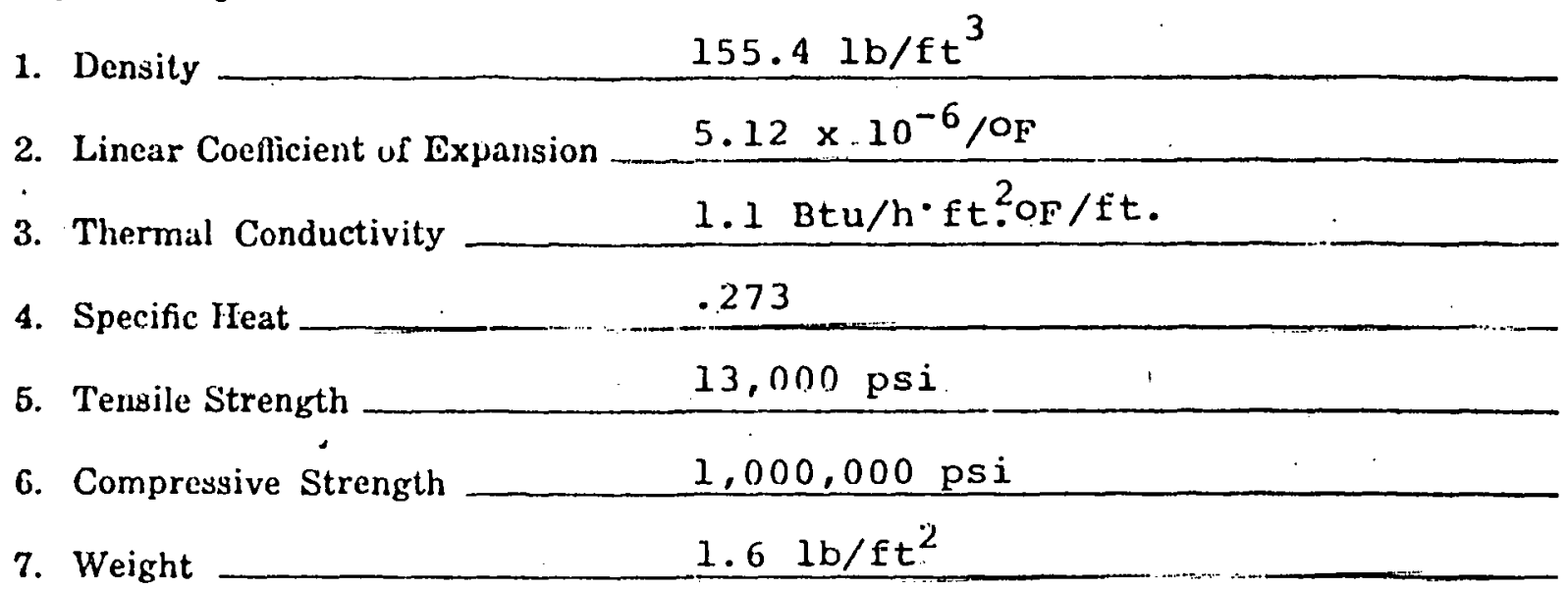

3. Absorber Plate

a. Absorptive Coating

1. Materials
a. Type

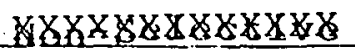

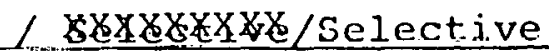

\footnotetext{
- Properties of conventional materials that are available in standard references such as Mark's Fingineering Handbook necd not be rcstated hese pruvided the naterial is adequately specified so that its properties can be determined from such references. Properties of materials not commonly available in standurd references should be submitted with system data to the extent known.
}

* at 1050 millimicrons 


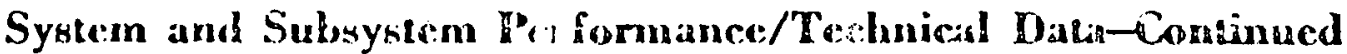

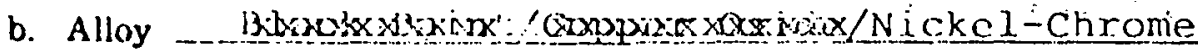

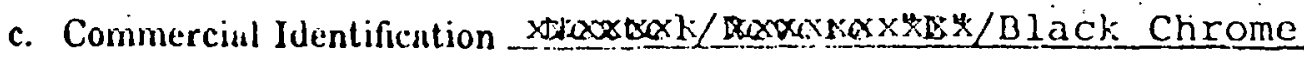

2. Solar Spectrum Absorptivity - $26 \% 20 \%$

3. Infrared Emisgivity $26 x^{2} x^{2} / k z a / 7$ $\%$

b. Base Plate

1.* Materials

a. Type Integral Tubes in Copper Plate

b. Alloy DHP Copper \#122

c. Commercial Identification Revere Tube-In-Strip

2. Thermal'Properties

a. Thermal Conductivity $\quad 196 \mathrm{Btu} / \mathrm{hr} \cdot \mathrm{ft}^{2} \mathrm{O}_{\mathrm{F}} / \mathrm{ft}$.

b. Specific Heat $0.0942 \mathrm{Btu} / \mathrm{Ib} \mathrm{O}_{\mathrm{F}}$

9. Physical Properties

a. Linear Coefficient of expansion $-9.3 \times 10^{-6} / O_{F}$

b. Density $5581 \mathrm{~b} / \mathrm{Ft}^{3}$

c. Tensile Strength $50,000 \mathrm{psi}$

d. Compressive Strength $38,000 \mathrm{psi}$

4. Bonding Materials
a. Type (Brazed, Soldered, etc.)
Brazed lleaders
b. Composition Sil-Fos or Phos Copper
c. Comnerical Identification Handy \& llarman or equal

4. Insulation

a. Materials

1. Type Blanket

2. Composition Piherglass

3. Commercial Identification Certain - Teed Ultralite 


\section{System and Subsystem Perforsance/Technical Data-Continued}

b. Outgassing Characteristics

1. Outgassing Temperature $450^{\circ} \mathrm{F}$

2. Gas given off Phenolic Resin

3. Any Condensation No

c. Physical Properties

1. Linear Coefficient of expansion. None

2. Density 1. $01 \mathrm{~b} / \mathrm{ft}^{3}$

3. Thermal Conductivity $0.27 \mathrm{Btu}-$ in $/ \mathrm{hr} \mathrm{ft}^{2} \mathrm{O}_{\mathrm{F}} \mathrm{a} .68^{\circ} \mathrm{F}$

4. Specific Heat Not Known

5. Coefficient of Cubical expansion None

6. Dimensious $76^{\prime \prime} \times 34^{\prime \prime} \times 2-1 / 2^{\prime \prime}$

5. Outer Base Enclosure

a. Materials

1. Type Extruded Aluminum

2. Composition $6063-75$

3. Commercial Identification Revere Aluminum

4. Combustibility $N / A$

b. Physical Properties (As Applicable)

1. Linear Coefficient of expansion $12.9 \times 10^{-6} / 0_{F}$

2. Density 1.68,6. Ab/fte ${ }^{3}$

3. Thermal Conductivity $1.19 \mathrm{Btu} / \mathrm{hr} \cdot \mathrm{ft} \mathrm{O}^{2} \mathrm{~F} / \mathrm{ft}$.

4. Sperific Heat 0.224

5. Coefficient of Cubical expansion $-N / \mathrm{N}$

6. Dimensions $77^{\prime \prime} \times 35^{\prime \prime} \times 4 "$

c. Thermal Conductivity $119 \mathrm{Btu} / \mathrm{hr} \cdot \mathrm{ft} \mathrm{2}^{20} \mathrm{E} / \mathrm{Et}$. 


\section{Sybtem and Subystem H"en formance/Terhaical Data-dostinued}

Passuve systems require that suflicion 1 calculations or test resulty to determine how effective the concepts will be in providing the neccessary functions. As a minimum, the following should be provided.

1. Test method used NBSIR 74-635 and DSET 75 SE 2

2. Energy Collection Rate ( $\mathrm{STU} / \mathrm{II}-\mathrm{ft}^{2}$ ) Versus time for selected winter conditions and (if applicable) for selected summer conditions over a collection day. The following: should be provided:

a. Collector Orientation
1. Azimuth
norinal to sun's rays
Degrees
2. Elevation nermal to sun's rays
Degrees

b. Ambient Conditions

1. Temperature see data ${ }^{\circ} \mathrm{F}$

2. Wind Velocity see data $\mathrm{MPH}$

3. Wind Direction _ase data Degree

c. Insolution

d. Collection Period (Time of Day) see data to

1. Provide Graph of Inlet Temperatures To obtain a distribution of data, the inlet temperatures were varied and are not a function of time.

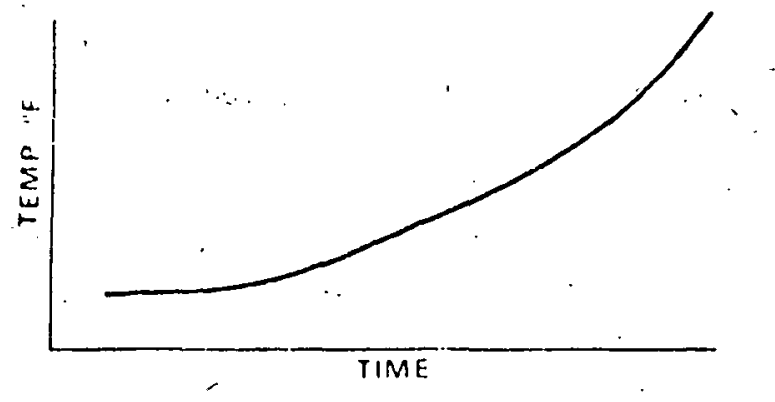

2. Provicie Graph of Outlet Temperaturen

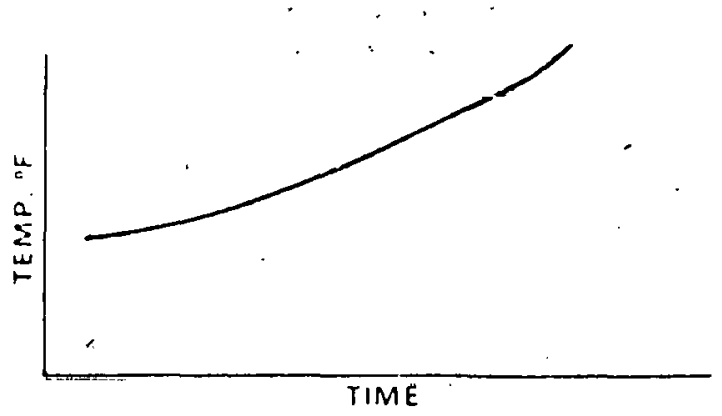

This is also the case for the outlet temperatures. The variation of inlet temperatures during the test does not permit a curve plot similar to that indicated above. However, the test data can be used to compute an inlet or outlet temperature graph for an assumed situation. 


\section{System and Subsystem Perfor:mance/Technical Data-Continued}

3. Provide a graph of Collector efficiency $(n)$ versus the parameter $\mathrm{Ti}-\mathrm{Ta}$.

$$
\text { where } n=\frac{M C_{n}\left(T_{0}-T_{1}\right)}{A_{c} I}
$$

See attached reports from Desert Sunshine Exposure Tests, Inc.

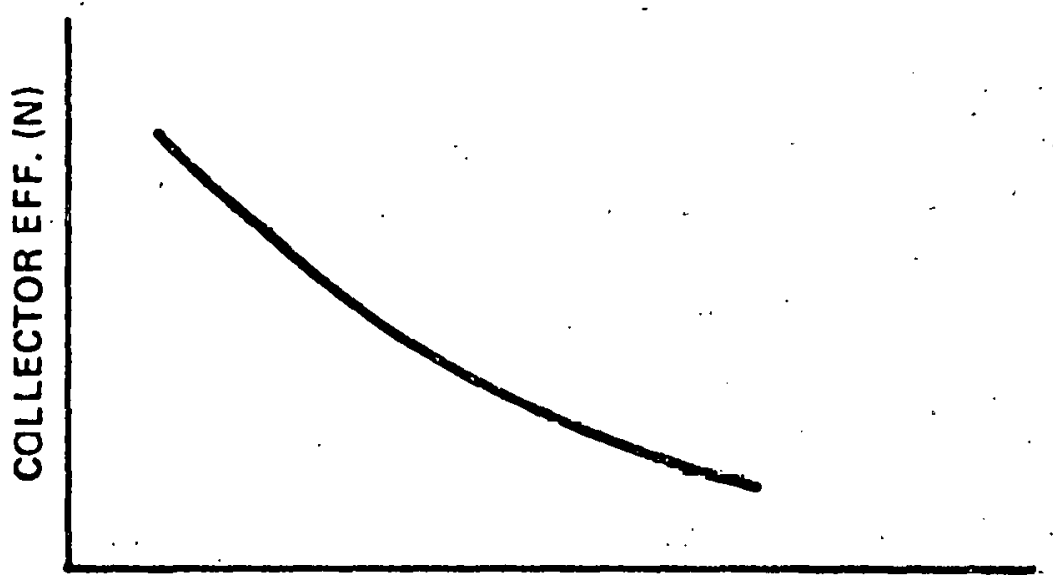

OPERATING PARAMETER

$$
\frac{T i \cdot T_{a}}{I}
$$

$\mathrm{T}_{\mathrm{n}}=$ Collector transport media outlet temperature $\left({ }^{\circ} \mathrm{F}\right)$

* $\mathrm{Ti}=$ Collector transport media inlet temperature $\left({ }^{\circ} \mathrm{F}\right)$

$\mathrm{Ta}=$ Ambient Temp. $\left({ }^{\circ} \mathrm{F}\right)$

* I = Solar Insolation on the Collector plane (BTU/HR - FT2)

$\mathrm{M}=$ Transport medla mass flowrate (lb/hr)

$\mathrm{C}_{\mathrm{n}}=$ Specific heat of transport media (BTU/LB ${ }^{\circ} \mathrm{F}$ )

$A_{c}=$ Area of Collector $\left(\mathrm{ft}^{2}\right)$

- Average Collector 'I'emp. may bo used $\frac{T_{1}+T_{0}}{2}$

* For concentrating collectors this value should be only the beam or direct component for the solar radiation.

4. Maximum expected temperature under no flow conditions $350^{\circ} \mathrm{F}$ *

5. Discuss provisions for protecting collector under no flow conditions.

6. Collector Array Characteristics

a. Total Area $18.6 \mathrm{ft}^{2}$

b. Solar Window Area $12.4 \mathrm{ft}^{2}$

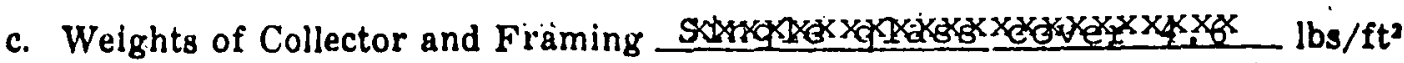
Double glass cover $6.1 \mathrm{ibs} / \mathrm{ft}^{2}$

* From Desert Sunshine data, no flow occurs at $\frac{\mathrm{T}_{f}-\mathrm{T}_{\mathrm{a}}}{\mathrm{q}}=.66$

If $q=350 \mathrm{Btu} / \mathrm{hr} \cdot \mathrm{ft}{ }^{2}$ and $\mathrm{T}_{\mathrm{a}}-120^{\circ} \mathrm{F}$, then $\mathrm{T}_{\mathrm{f}}=350^{\circ} \mathrm{F}$ 


\section{Symtem and Subsystem Performance/Trechnical Data-Continued:}

(.) Storiage

1. Type (Tank, Rock Bed, etc.) TANKS

2. Matcrials
«. Type
STEEL
b. Pinishes
INTERIOR COAL TAR EPOXY
c. Commercial Identification

3. Ihysical Dimensions: THREE TANKS, EACH:
a. IIeight
92" DIAMETER
b. Width
$-$
c. Length
$14 \div-10 "$

4. Thicrmal Properties*

a. Thermal Conductivity (WATER)

b. Coefficient of Thermal expansion

(WATER)

5. Operating Temperature Range $40-200$

6. Opcrating Pressure Range* $15-25$

7. Burst Pressure* 60 (Min.)

D. Cooling Sulsystem

1. 'Type CHILLER

2. Commercial Unit

a. Type ABSORPTION

b. Size 25 (WITII $195^{\circ}$ IIOT WATER ENTENING)

c. Identification ARKLA SOLAIRE 300

3. Materials

i. Types

b. Commercial Identification

- Propertics of conventional materials that are available in standard references such as Mark's Engineering Handbook need wot be restated here provided the nanterial is adequntely specified so that its properties can be determined from such reforme's. Froperties of materials not commonly available in standard references should be subnitted with system data to the: extent linown. 


\section{System and Subsystem Performance/Technical Data-Continued}

1. Fluids
a. Types
WATER
b. Composition

5. Coefficient of performance (COP) data versus pertinent operating conditions (ambient temperature etc.) along with a definition of the COP used.

C.O.P. = Output $(\mathrm{Btu} / \mathrm{Hr}) /$ Input $(\mathrm{Btu} / \mathrm{Hr})=0.6$

6. Total Cooling Capacity

Total cooling capacity of the solar system shall be no less than 196,300 BTU/HR (if it is a heating and cooling system). Sensible capacity shall be no less than 166,800 BTUं/HR at $8000 \quad \mathrm{CFM}$ of entering evaporator air at 80.0 OF dry bulb and 67.0 $\circ \mathbf{F}$ wet bulb. For other systems such as desiccant cycling cooling, the terms evaporator and condensor are not applicable. 'These.systems shall deliver the above cooling capacity at inlet air flow of N.A. CFM at ${ }^{\circ} \mathrm{F}$ dry bulb and N. N.___ ${ }^{\circ} \mathrm{F}$ wet bulb.

E. Heating Subsystem

1. Type SOLAR WITH BOILER FOR BACKUP

2. Commercial Unit
a. Type GAS FIRED WATER TUBE
b. Size 440 MBH OUTPUT
c. Commercial Identification
RITE

3. Coefficient of Performance (COP, if applicable, data versus pertinent operating conditions (ambient temperature etc.) BOILER EFFICİENCY 80 \%

4. Total Heating Capacity

'The total heating capacity of the solar system shall be no' less than 233,000 BTU/HR at BD०0 CFM of air flow entering at 65 ${ }^{\circ} \bar{F}$ dry bulb and $\%$ relative humidity. Exposed heated panel (baseboard or ceiling) temperatures shall not exceed $\mathrm{N}: \mathrm{A} .{ }^{\circ} \mathrm{F}$.

F. Hot Water Subsystem

1. Type

DOMESTIC WATER HEATER 


\section{System and Subsytem Performance/Technical Data-Cóntinued}

$\therefore$ Commereial Unit
a. Type
1. Sire 66 GAL.
¿. Commercial Identification RHEEM SOLARAIDE

:1. Hot Water (Back Up System) : 66 _ gallons of potable (of useable) hot water shall be delivered at no less than _.___ 8 gal/min at temperature no less tham 125 _ oF. Recovery time shall be no greater than 2

1. Civle and Safoty Standard Certified Under UNDERWRITERS' LABORATORIES

G. Trunsport Bictucen Subsystims

1. Provide Sketch/Block diagram of Proposed Solar System giving dimensions and subsystems! components location and jomtification. NOTE: This project is in preliminary design \& physical layout has not been established: See schematic

2. I'iping Details Flow Diagram for functional relationship of components.
a. Diameter
b. Length of Run
c. Materials COPPER

3. Piping Insulation
a. Type FIBERGLASS
b. Thickness $I^{\prime \prime}$
c. Performance $\mathrm{K}=0.26 \mathrm{AT} 200^{\circ} \mathrm{F}$

4. Transport Media for each element
a. Type WATER
b. Flow Rate GPM (Liquid) CFM (Air)
c. Specify Pressure drop between components.

5. Provide Flow diagram for Proposed Solar Energy System. See "SCHEMATIC FLOW DIAGRAM" .

IL. System.

\section{Operating. Requirements}

a. The maximum electrical energy required to drive the solar portion of the system at its rated 


\section{System and Subsystem Performance/Technical Data-Continued}

capacity shall be no greater than 5

K.W. Water requirements for cooling condensers nnt/or arrehmitifeation shall be no greater than $100 \quad \mathrm{gal} / \mathrm{hr}$.

b. Subsystems/Components requiring electrical energy :

1. Pumps $2 \ldots \mathrm{kw}$, Function_CHILLED WATER

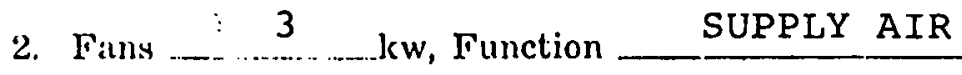

3. Controls $0.5 \quad \mathrm{kw}$, Function AIR COMPRESSOR

4. Other ______ $\mathrm{kw}$, Function $---$

2. Design Load Data:

ANNUAL SUMMARY TABLE

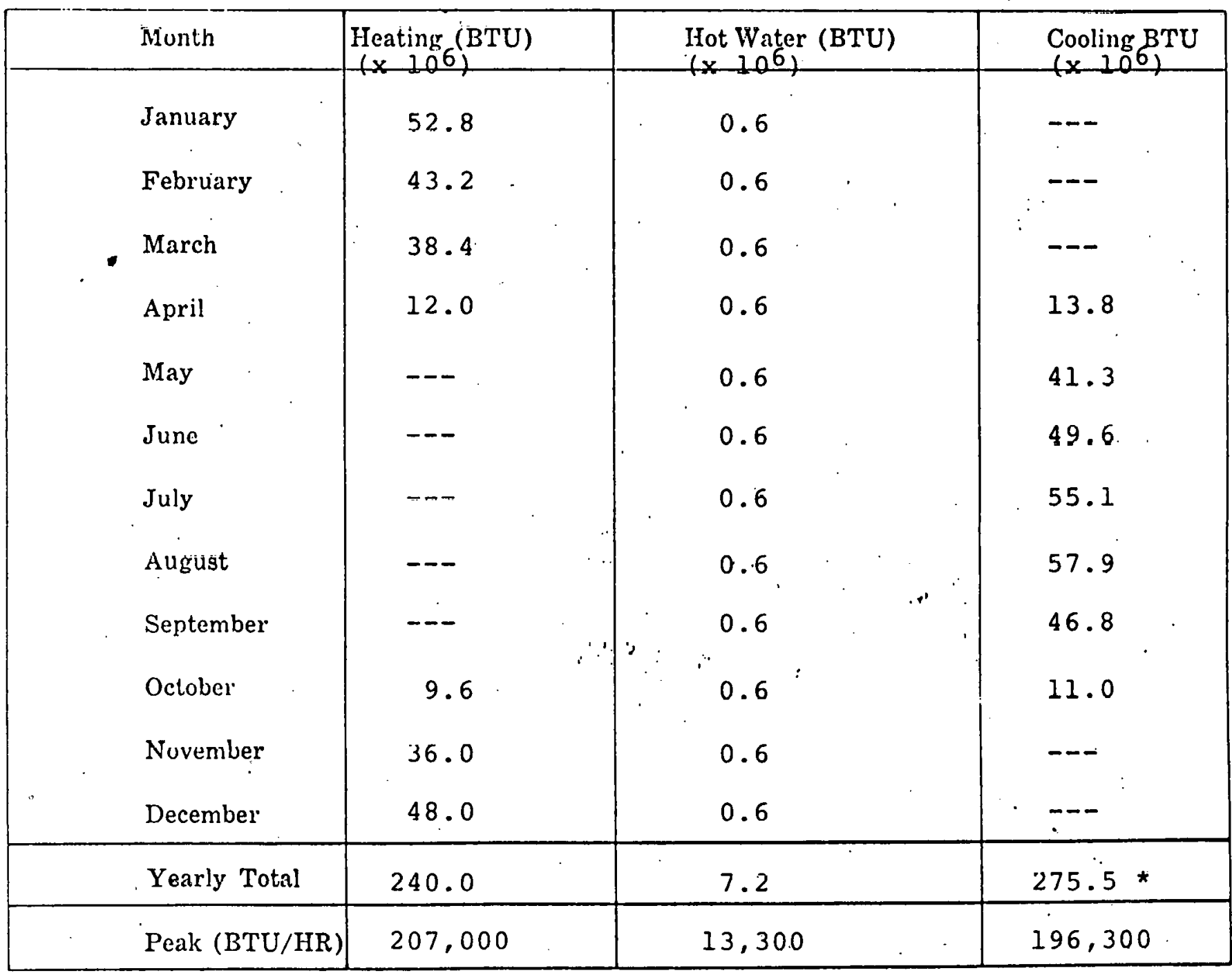

*Calculated Building Cooling Requirement; Divide by 0.6 for Chiller Input (System Heat Loss) 


\section{System and Suhsystem Performance/Technical Data-Continued}

3. P'rovide the following summary of system perfornance data:

\begin{tabular}{|c|c|c|c|c|c|}
\hline $\begin{array}{c}\text { Month } \\
-\quad-\quad\end{array}$ & $\begin{array}{l}\text { Solar Fonergy } \\
\text { (onllected } \\
\left(B^{\prime} l y\right) \\
\left(x \cdot 10^{6}\right) \cdots\end{array}$ & $\begin{array}{l}\text { Iislectrical } \\
\text { Fuserry Recp'd } \\
\text { Sor (omponent } \\
\end{array}$ & 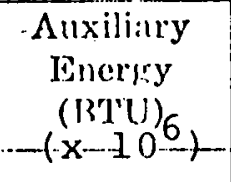 & $\begin{array}{l}\text { System IJeat } \\
\text { Joss (BTU) } \\
-(x-1.06)\end{array}$ & $\begin{array}{l}\text { Equivalent Enorey } \\
\text { lieg'd for Conven- } \\
\text { tionial Sritgm (B'lu) } \\
\text { (xibs) }\end{array}$ \\
\hline J:tnuatry & 42.2 & 2320 & 11.2 & 53.4 & 53.4 \\
\hline lialuwally & 43.2 & 2320 & .6 & 43.8 & 43.8 \\
\hline March & 39.0 & 2320 & -- & 39.0 & 39.0 \\
\hline$\Lambda_{\text {puil }}$ & 35.6 & 2320 & -- & 35.6 & 23.5 \\
\hline Mily & 39.3 & 2320 & 30.1 & 69.4 & 41.9 \\
\hline June & 43.1 & 2320 & 40.2 & 83.3 & 50.2 \\
\hline July & 46.9 & 2320 & 45.5 & 92.4 & 55.7 \\
\hline August & .44 .9 & 2320 & 52.2 & 97.1 & 58.5 \\
\hline September & 42.9 & 2320 & 35.7 . & 78.6 & 47.4 \\
\hline October & .40 .9 & 2320 & 4.6 & 45.5 & 21.2 \\
\hline November & $\ldots .36 .6$ & 2320 & -- & 36.6 & 36.6 \\
\hline Decomber & 43.0 & 2320 & .5 .6 & 48.6 & 48.6 \\
\hline
\end{tabular}

$\therefore$ Provide estimate of yearly energy savings in terms of BTU's and/or Dollars along with the rationi:le for the estimate.

$(519.8-225.7)\left(10^{6}\right) \div 3413=86170 \mathrm{KWH} \quad \mathrm{x} 0.04=\$ 3445 / \mathrm{Yr}$

5. Any subsystems or system energy conversion inefficiencies which have not been specified in the previous: subsystem section should be provided now. For example, if an oil fired heater is used for an auxiliary encrgy source state its:

1. Commercial identification GAS FIRED BOILER SPECTFIED PREVIOUSLY.

2. Size/Rating (BrCU)

3. Lificiency -0.80

4. Electrical Power Requirements

6. Provide summary of insolation clata used for section H Analysis. See "SUPPORTING INFORMATION" . 


\section{System and Subsystem Performance/'Technienl, Daln-Continued}

7. Design Life and Maintenince

a. Describe Periodic Mainlenance provisions and reçuirenents.

b. Specif y design life of all components (if avirilible).

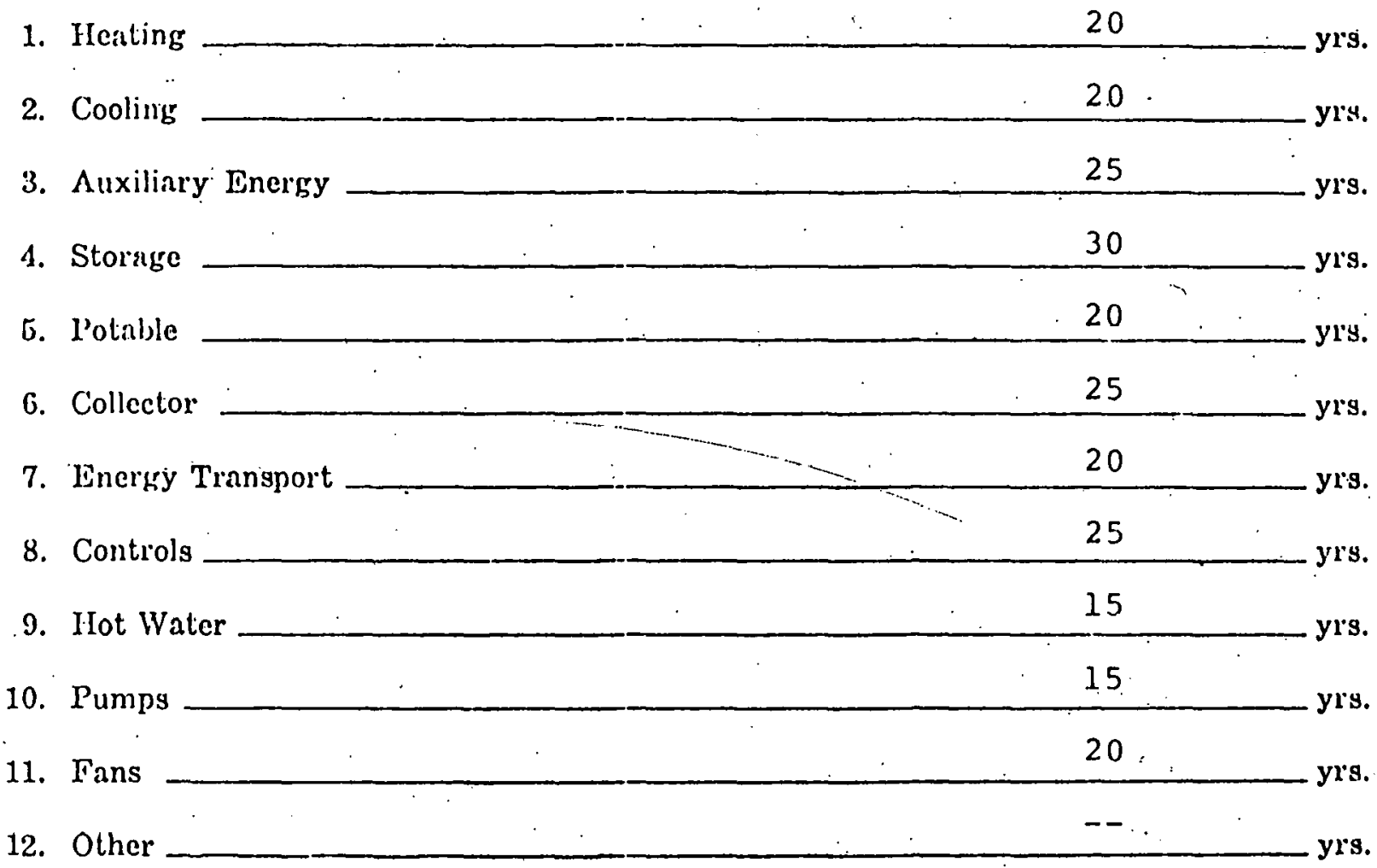

c. Provide Warranty period and extent of coverage of the proposed Solar Energy System and subsystems.

Contractor will provide a one year warranty on materials and workmanship, which is the normal under a general construction contract. In addition an extended warranty, for a total of five years, will be provided for major system components. 
The installation will. comply with the intent of the "Interim Performance Criteria for Commercial Solar Heating and Combined Heating/Cooling Systems and Facilities", NASA \#98M10001, February 28, 1975. 
Assumptions:

Collectors at 500. from Horizontal : :

Average Daily Insolation $2150 \mathrm{Btu} / \mathrm{Ft}^{2} \pm$

Approximate 8 sunshine $\underline{58}$

Average Hourly Insolation $=0.58 \times \frac{2150}{11 \times 0.9}=126 \mathrm{Btu} / \mathrm{Sq} \cdot \mathrm{Ft} . \mathrm{Hr}$.

Average Ambient Temperature $45^{\circ}$

Flow Rate $=1 \mathrm{GPH} / \mathrm{Sq} \cdot \mathrm{Ft}$.

Average Collector Efficiency $55 \%$

Average Output $69 \mathrm{Btu} / \mathrm{Hr} . \mathrm{Ft}^{2}$ for 8 Hours

(All available for storage when building is occupied)

Average Daily Unoccupied Heat Loss (January):

$1,153,000$ Btu

Sq. Ft. Collector Required $=\frac{1,153,000}{8 \times 69}=2090 \mathrm{Sq} \cdot \mathbf{F t}$. 
Based on collectors at $50^{\circ}$ to horizontal, reflector augmented, $190^{\circ}$ leaving water, estimated energy available is $880 . \mathrm{Btu} / \mathrm{Sq}$. Ft. per day (August):

Square Feet of collector required to handle peak cooling load:

$$
\frac{328,000 \mathrm{Btu} / \mathrm{Hr} \text { Input }}{100} \quad 3280 \mathrm{Sq} \cdot \mathrm{Ft} .
$$

Physically practiçal array:

5 rows $\times 20$ panels/row $\times 17.4$ Sq.Ft. Aperture $=1740$ sq.Ft.

Estimated peak collection rate (average for day):

$\frac{1720 \mathrm{Sg} \cdot \mathrm{Ft} . \times 880}{12 \times 0.9}=140,150 \mathrm{Btu} / \mathrm{Hr}$

438 of Peak Requirement

Collection for Peak Month:

$1720 \times 880 \times 31=46.9 \times 10^{6}$ 
Roof $66^{\prime} \times 62^{\prime} \times\left(70^{\circ}-5^{\circ}\right) \times 0.13=34,575$

Walls $(256 \times 24)(85 \%)(0.2)(650):=67,890$

Glass $(256 \times 24)(15 \%)(0.6)\left(65^{\circ}\right)=35,940$

Floor Perimeter $256 \times 50=\underline{12,800}$

Transmission Subtotal 151,205 Btu/Hr

Outside Air $800 \times 1,08 \times 65=\ldots .56,160$

Calculated Heat Loss $=\quad 207,365$

$\frac{207,365}{6000}=34.5 \frac{\mathrm{Btu}}{\mathrm{Hr} . \mathrm{Ft}^{2}}$ Conditioned Area

Specify Minimum Heating Capacity of $207,365 \times 120=$ $248,840 \mathrm{Btu} / \mathrm{Hr}$ 


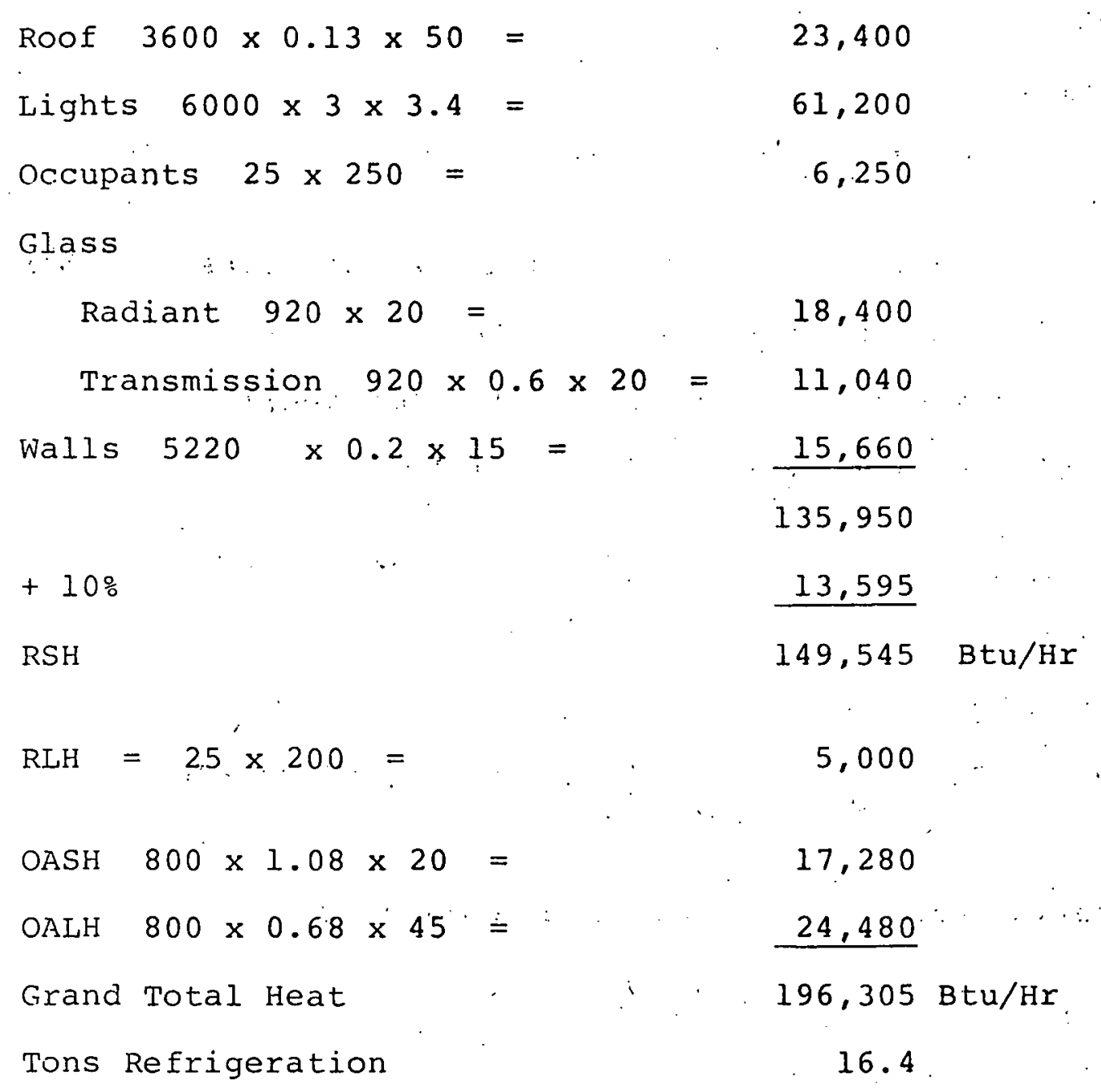


OCCUPIED HOURS

(October - April)

7 Mo. $\times 4.32 \frac{\mathrm{Wks}}{\mathrm{MO}} \times 50 \frac{\mathrm{Hrs}}{\mathrm{Wk}}=1510$ Hours

Average Outside Temperature During Occupied Heating Hours: $57.5^{\circ} \mathrm{F}$

Average Heat Loss From Building (Occupied Hours)

(Assume No Heat Loss from Building above $65^{\circ} \mathrm{F}$ outside):

$207,365 \frac{\mathrm{Btu}}{\mathrm{Hr}}$ Design Loss $\times \frac{(65-57.5)}{(70-10)}=25,920 \frac{\mathrm{Btu}}{\mathrm{Hr}}$

Annual Heating Requirement, Occupied Hours:

$$
25.92 \times 1.510 \times 10^{6}=39.1 \times 10^{6} \text { Btu }
$$

Credit for Lighting:

$66-2 / 3 \% \times 6000 \mathrm{Sq} \cdot \mathrm{Ft} \cdot \mathrm{x} \frac{3 \mathrm{~W}}{\mathrm{Sq} \cdot \mathrm{Ft} .} \times \frac{3.4 \mathrm{Btu}}{\mathrm{W}}=40,760 \mathrm{Btu} / \mathrm{Hr}$

Net Annual Heating Requirement, Occupied Hours $=0$

UNOCCUPIED HOURS

$(7 / 12 \times 365 \times 24-1510)=3600$

Average Outside Temperature During Unoccupied Hours: $42.5^{\circ} \mathrm{F}$

Average Heat Loss from Builaing (Unoccupied Hours, Lights off, Inside Temperature set Back to $60^{\circ} \mathrm{F}$ ):

$$
207,365 \times \frac{(60-42.5)}{65}=55,830 \frac{\mathrm{Btu}}{\mathrm{Hr}}
$$

Annual Heating Requirement, Unoccupied Hours:

$$
3600 \times 55,830=201 \times 10^{6} \mathrm{Btu}
$$


Peak Energy Input to Chiller =

$$
\frac{16.4 \text { Tons } \times 12}{0.6 \text { (Average } \frac{000}{.0 . P .)}}=328,000 \mathrm{Btu} / \mathrm{Hr}
$$

Heat Equivalent of HVAC Auxiliaries, Cooling Cycle: $10.5 \mathrm{KW} \times 3413=35,835 \mathrm{Btu} / \mathrm{Hr}$

Average Monthly Energy Used by Auxiliaries:

17 Days $\times 13$ Hrs/Day $\times 35,835=7.9 \times 10^{6}$ Btu 
SOURCES

-- Heating degree days, equivalent full load operating hours: 1976 ASHRAE SYSTEMS HANDBOOK, Chapter 43.

-- Outside design conditions, transmission and solar load factors: 1972 ASHRAE FUNDAMENTALS HANDBOOK.

-- Daily solar inradiation to $45^{\circ}$ collector surface: 1974 ASHRAE APPLICATIONS HANDBOOK, Chapter 59.

-- Average summer and winter insolation values: "Monthly Maps of Mean Daily Insolation for the United States", SOLAR ENERGY，9，(3)，pp 164-165 (1965).

-- Normal daily maximum and minimum temperatures, mean percentages of possible sunshine: ITT SOLAR HEATING SYSTEMS DESIGN MANUAL.

-- Collector performance: Manufacturer's published data.

-- "Design and Simulation Studies for the Shenandoah Community Center Large Scale Solar Cooling Demonstration" (ASME Publication). 


\section{PART 7}

PROBLEMS

The North Georgia APDC solar system has operated continuously for eight months with a total down time (system out of commission) of approximately a week. Partial down time, where a subsystem malfunctioned, has been about six weeks. During the eight month period, the system has either effectively delivered the required heating or cooling from solar or from the back-up (supplementary) system, or as in one instance, during which the boiler was inoperative, heating was provided exclusively by the solar system.

The most significant problems encountered during the eight month operational period are the freezing of the collectors, failure of the main thermal circulation pump motor, and malfunction of the ignitor transformer on the boiler. Most problems, with the possible exception of the freeze damage to the collector, appear to be material failure and not necessarily asșociated with any design inadequacy. All problems have been handled in an expeditious manner by the design-installation contractor.

A detailed discussion of these problems is presented below.

1. System Freeze: Over the weekend of December 9-10, the North Georgia area experienced a sharp drop to below freezing temperatures, i.e., from $70^{\circ} \mathrm{F}$ to $10^{\circ} \mathrm{F}$, as a rapidly moving cold front moved through the area. The cold frontal passage was preceded by record high temperature on December 7-8. During the freeze, period the solar collector array experienced severe freeze damage to the supply headers. The extent of freeze damage was limited to the supply headers (primarily the two headers supplying the center collectors) where approximately 44 leaks were discovered. In addition, 4 leaks were found in one top return header, and 2 leaks in the bottom of one collector. Although 
the freeze damage probably occurred on the weekend, the system appeared to operate satisfactorily on the 11 th (operating on supplementary boiler). The first malfunction was noted on the morning of the 11th, i.e., the failure of a dry seal in the main circulation pump in the solar loop. A complete damage evaluation was conducted on December 13, 14, 15th. The system operated on supplementary boiler for approximately one week while repairs were made to the collector array.

A verified explanation for the system freeze has not been completely developed. The most reasonable explanation is that water vapor from the hot water storage tank was admitted to the system through the solar collector return lines by a siphoning action. The NGAPDC system employs an automatic draindown feature and if the return line was above the water level in the thermal storage tank, water vapor could follow this route into the collector. When the rapid change in outside temperature occurred, it is theorized that condensation in the collectors produced sufficient vacuum to drain water into the system, then freeze and produce the damage. System modifications that have been incorporated in the system as a result of the freeze were the installation of air vents on the top of each return header on the collector array and adding more water to the storage tank so that the collector return line was submerged below the tank̄ water level.

2. Failure of the main collector circulation pump motor: The removal of the motor for repair under the warranty arrangement required over three weeks: During this period, heated water was supplied by the boiler (creating extra expense for the additional fuel oil) but the building remained comfortably cool. This situation could have ben avoided by having a spare motor on hand.

3. Boiler: During the period January 15-25, the supplementary heating system (oil fired boiler) developed problems in the ignition system. Once 
the boiler had shut down on automatic control, it would not reignite. During this period, the boiler was started manually for heating the building in the morning hours. For approximately three days the building was heated strictly by solar. The trouble with the boiler was traced to the igniter transformer, which was replaced.

4. Instrumentation: The design of the North Georgia APDC solar system did not specify monitoring instrumentation, and from all overall design standpoint, this has been a serious omission. The owner-operator of the system has found that a qualitative assessment of the operation of the system is impossible. The system provides the correct level of heating and cooling as required; however, any measure of operational efficiency is unavailable. In addition, the isolation of problems by the operator-owner has been made more difficult without this instrumentation. Hopefully, this situation will be corrected.

5. Miscellaneous: During the first few weeks of operation, operating controls, set points on thermostats, and balancing the various zones was a minor problem but obviously not an unexpected one. Minor adjustments have been required during the change over to fall weather, however, it is questionable whether this is a problem area. In all cases, the contractor responded to make appropriate adjustments. 
PART 8

RECOMMENDATIONS

The North Georgia: APDC solar system has proved to be an efficient, cost effective system primarily due to the special efforts of all members of the project team. It is indeed difficult to raise problem areas when the overall project is a success, however, it is our recommendation that future grantees pay very close attention to the development of the team, the lines of authority which are required in contractual arrangements and aspects of the solar field which are still experimental. It has been our experience that the owner-operator establish the appropriate line of contractual authority as soon as possible, so that all parties can perform effectively from an established position.

In the opinion of the owner-operator, the success of the North Georgia APDC solar system can be attributed to effective operational design. However, another important aspect of design was to specify quality materials and workmanship which has provided a relatively trouble-free system.

The design specified copper collectors, copper plumbing, welded steel collector array support, steel storage tanks, high quality pumps and valves, and quality control on all solder joints. In addition, all pipes and exposed surfaces are fully insulated, including exposed portions of the chiller. This attention to quality has resulted in an outstanding system.

Another noteworthy feature of the North Georgia APDC is the prefabrication process used by ILI. All major components such as the collector modules, reflector panels and the control module were preassembled off-site. These components were delivered to the site at the, appropriate time for installation. In this way, installation of the system was completed in approximately four weeks with a minimum of difficulty. This also has attributed lo the soundness of the system. 


\section{PART 9}

VERIFICATION STATEMENT

9-1 


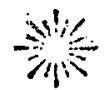 \\ $\pi \square$}

\section{Independent Living, Inc.}

5965 Peachtree Corners East

Norcross, Georgia 30071 / Tel. (404) 449-5900

March 14, 1979

North Georgia Area Planning \&

Development Commission

503 West Waugh Street

Dalton, Georgia 30720

Attention: $\mathrm{Mr}$. Don Kolberg

Dear Mr. Kolberg:

The solar system installed at your facility in Dalton was installed per the "as built drawings" provided through your organization.

The system performed in accordance with the test plan provisions at the time of the System Acceptance Test in Dalton.

The system meets the intent of the interim performance criteria for solar commercial heating and cooling and heating/cooling systems, NASA document \#98M10001, dated February 28, 1975.

Sincerely,

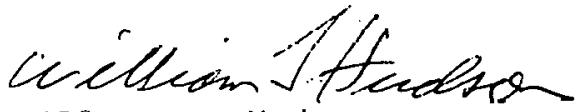

Wili iam T. Hudson

President

WTH : lup 
APPENDIX A

MANUFACTURERS LITERATURE

SYSTEM COMPONENTS

$10-1$ 


\section{General Description}

Arkla's Solalire: 300 wate chiller is designed primarily. for solar cooling applications but can be used tot a broad range of comfort air condition ing and industrlal process appllcations. With tiring water temperature between $160^{\circ} \mathrm{F}$. and $200{ }^{\circ} \mathrm{F}$, and with $85^{\circ} \mathrm{F}$ conderising water, llie ma chine can produce from 7.5 tons to 26.5 tons of cooling capacity

\section{Features}

9. The unit is nominally rated at 25 tons, but design flexibility allows easy adaotation for a wide range of capacities

- The special lithilim bromide Water solution is instafled at the factory and each machine is given a complete capaclty tost.

- The low concentration of the solution reduces the possibility of crystallization. ta an insignificant status.

- The hermetically sealed centrifugal pump is magnetically diven. This oump transfers solution from the Inw pressare side to the high pressure side of the absorntion cycle

- The combination of the solution oump and an abin: darce of heat ranster su? face permits this whit to produce piactical conling: capacity from relatively low firing water temperatures:

- The generator tubes and other vital areas are con: structed of type 304 stain less steel to insure years of trouble free service,

\section{Operating Controls}

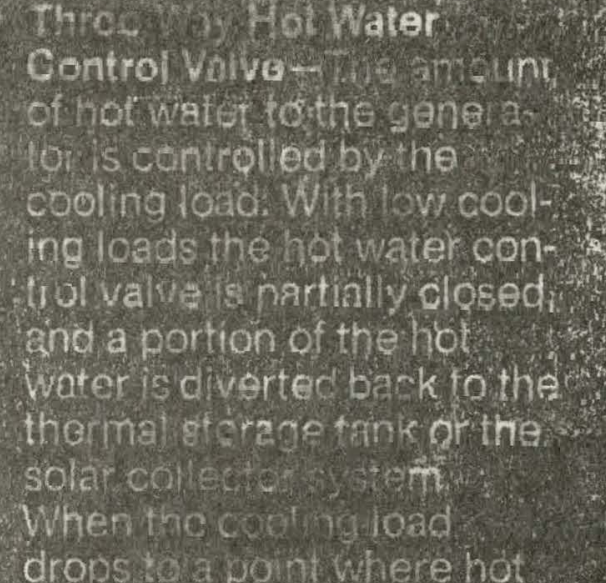

\section{wat}

valve closes and an end

switch will cause the unit

to shut down

- Two Input Coniroller - With two temperature sensing 6 bulbs one tor leaving chilled waterand one $10 \mathrm{c}$ returning chilled wate this solia state device contro/s the operation of the three. way hot water control valve.

- Solution By Pass Valve and Timer $=$ On start-up, this valve opens causing solf tion to by-pass the absorber for two minútes. Otherwise, excessively tow femperas tures could be oroduced intil the chiller is presented la load

o concentraton Chamber Dun Valo m it that and thure of tio condensing Water tails a ororox matay

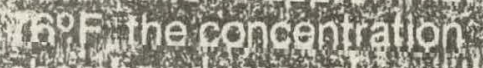

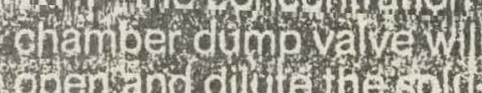
2.

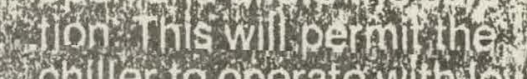
dhilrer to operate with 6 ow condensing water temok catures.

- Pump Control Relays Three relays are provice of to eneraize motor stanters. for chilled water hot water and condensing water circulating pumps:

\section{Safety Controls}

REvaporalor Low Tempera: - ture switch-it the refriger tent in the flash chamber Talls below mininum temperatures, this safely switch Will cause the hot water Mcontrol valve to close and divertall the hot water around the generator. It will

(4) Talso shet down both the solution pump and the condensing water pump

- Chilled Water LoW Temper- ature switch-This safely switch performs the same Xfunction as the evaporator Low temperature switch but wenses the temperature of Wheleaving chiled water - 6ondenser High temperaVure Limit swilch if the Temperature of the condense rises above accep7 ablalimits, this satety - switch will also cause the hot water control valve to close and divert the firing water around the generator , Chilled Water Flow Switch 4-it the trow of chilied wate Weturning to the chiller falls below established mini: nums this safety switch will shum cown the grachine in the same manner as the Xvapotor low femperature

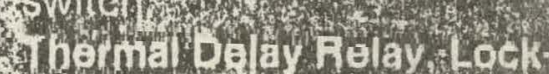
tut iela

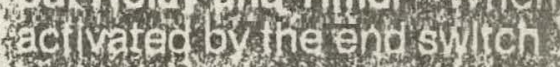
Th the Ho Nar cojtro Talve, tosse contros will

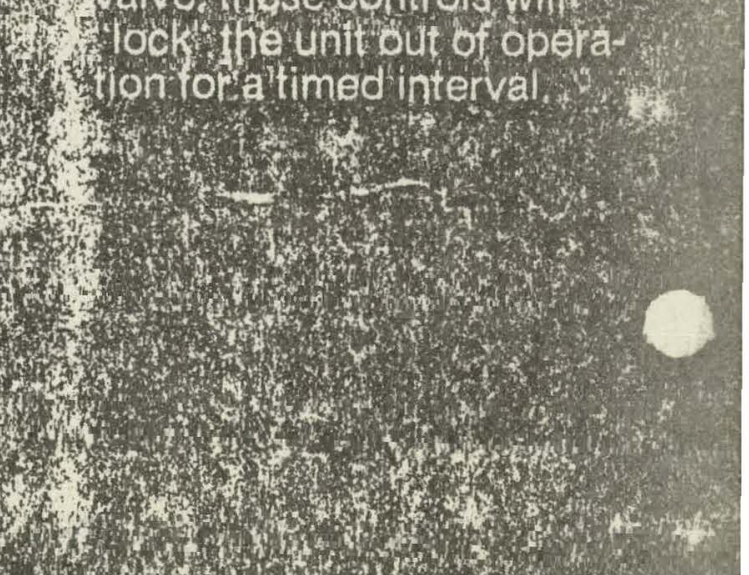




\section{SPECIFICATIONS}

MODEL. WFB 300

DESIGN DELIVERED CAPACITY, Btu/h. . . . 306,000

DESIGN DELIVERED CAPACITY, Tons I.M.E.... 25.5'

\section{ENERGY REQUIREMENTS}

Design Hot Water Input, Btu/h . . . . . . . . . . 447,000

Design Hot Water Inlet Temperature, ${ }^{\circ} \mathrm{F} \ldots \ldots \ldots .195$

Design Hot Water Outlet Temperature, ${ }^{\circ} \mathrm{F}$. . . . 184.8

Permissible Range of Inlet Temp......... 160 to 200

Design Hot Water Flow, gpm............... 90

Pressure Drop, Feet of Water, at $90 \mathrm{gpm}$. . . . . . 20.7

Permissible Range of Flow. gpm........ 50 to 100

Pressure Drop, Feet of Water, at $100 \mathrm{gpm}$. . . . . 25.6

Maximum Working Pressure, psig ............ 100

Electrical Voltage, $60 \mathrm{~Hz}, 1$ Phase . . . . . . . . . . . 1152

Maximum Wattage Draw................. 150

\section{CHILLED WATER DATA}

Design Inlet Temperature, ${ }^{\circ} \mathrm{F} \ldots \ldots \ldots \ldots \ldots \ldots \ldots 55$

Design Outlet Temperature, ${ }^{\circ} \mathrm{F} \ldots \ldots \ldots \ldots \ldots .45$

Design Flow, gpm...................6 60

Pressure Drop. Feet of Water, al $60 \mathrm{gpm}$. . . . . . 9.8

Permissible Range of Flow, gpm ......... 30 to 100

Pressure Drop, Feet of Water, at $100 \mathrm{gpm}$. . . . . 26.9

Maximum Working Pressure, psig ........... 100

Unit Water Volume, Gallons, Approx. . . . . . . . . . . 12

Fouling Factor . . . . . . . . . . . . . . . . . 0005

\section{CONDENSING WATER DATA}

Design Heat Rejection. Btu/h . . . . . . . . . . 753,000

Design Inlet Temperature, ${ }^{\circ} \mathrm{F} \ldots \ldots \ldots \ldots \ldots \ldots . . . \ldots 5$

Design Outlet Temperature, ${ }^{\circ} \mathrm{F} \ldots \ldots \ldots \ldots \ldots .101 .7$

Permissible Range of Inlet Temp. . . . . . . . 75 to 90

Design Flow, gpm . . . . . . . . . . . . . . . 90

Pressure Drop, Feet of Water, at $90 \mathrm{gpm}$. . . . . . 22.9

Permissible Range of Flow, gpm ........ 50 to 110

Pressure Drop, Feet of Water, at $110 \mathrm{gpm}$. . . . . 33.5

Maximum Working Pressure, psig ............ 100

Unit Water Volume, Gallons, Approx. . . . . . . . . . . . 20

Fouling Factor . . . . . . . . . . . . . . . . .

\section{FOR COOLING TOWER SELECTION}

Maximum Heat Rejection, Btu/h . . . . . . . . 853,000

Range ${ }^{\circ} \mathrm{F} \quad \ldots \ldots \ldots \ldots \ldots$ to 17

Minimum Permissible Sump Temperature, ${ }^{\circ} \mathrm{F} . \ldots 75^{3}$

\section{SERVICE CONNECTIONS}

Hot Water Inlel and Outlet . . . . . . . . . . . . . 2" FPT

Chilled Water Inlet and Outlet . . . . . . . . . . . 21/2" FPT

Condensing Water Inlet and Outlet....... 2 $\frac{1}{2}$." FPT

\section{PHYSICAL DATA, APPROXIMATES}

Operating Weight, Pounds...... . . . . . . . . . 3,4204

Shipping Weight, Pounds . . . . . . . . . . 3,145

Crated Size. Inches. . . . . . . . . . 114 W, 45D, 69H

NOTES: 1. Capacity at design conditions. For capacities at other conditions, see Page 4.

2. Units equipped for operation on $230 \mathrm{~V}-50 \mathrm{~Hz}-1 \mathrm{Ph}$ available on special order.

3. Thermostatic switch to control tower fan MUST be used. Set lo "cut out" at $75^{\circ} \mathrm{F}$.

4. Includes circulating water weights.

5. Units as shipped contain Lithium Bromide charce.

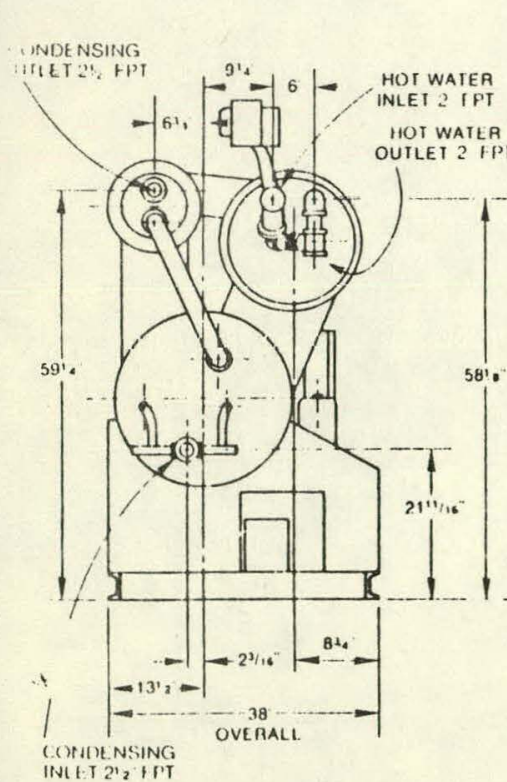

LEFT VIEW

\section{DIMENSIONAL VIEWS}

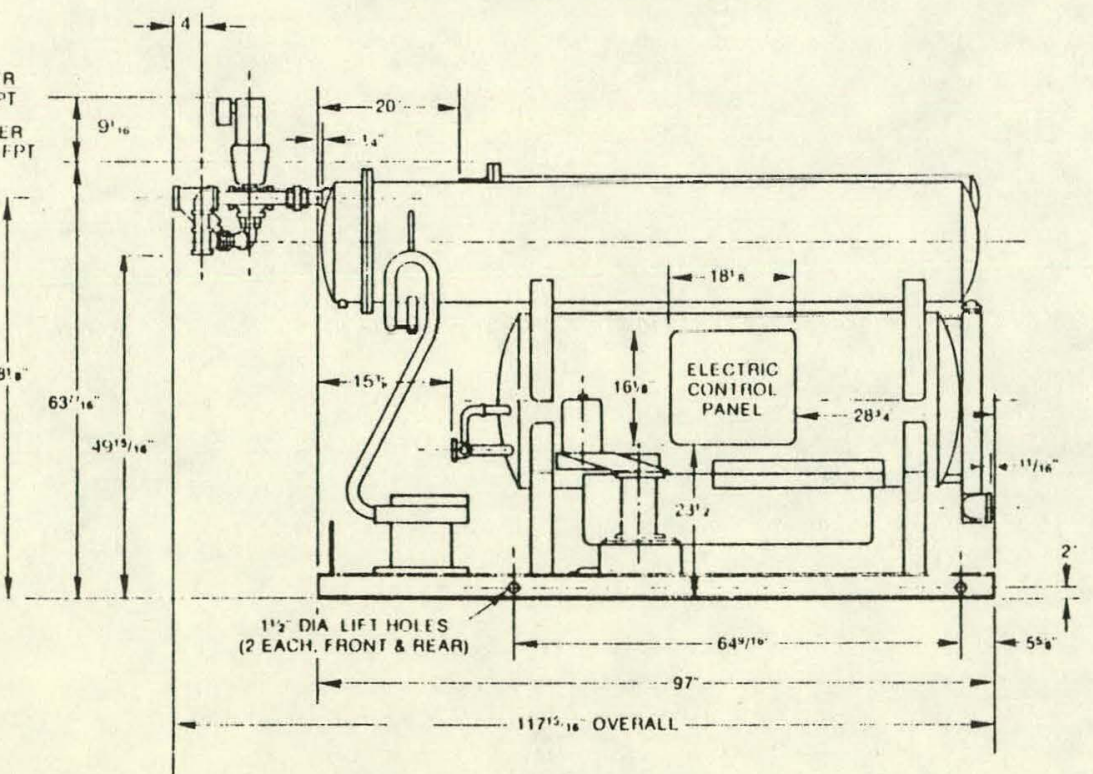

FRONT VIEW

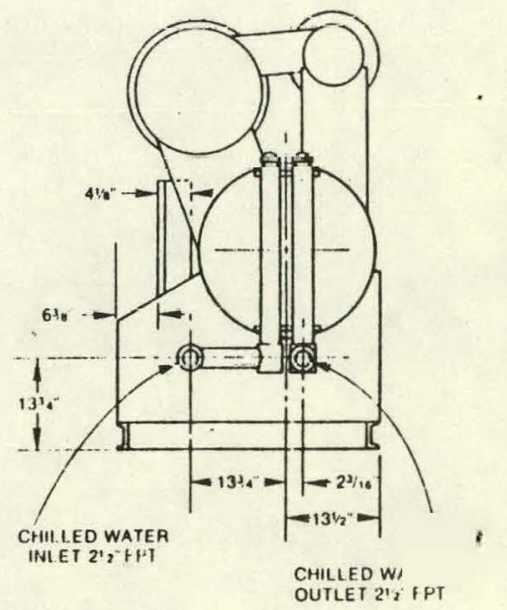

RIGHT VIEW 


\begin{tabular}{|c|c|c|c|c|c|c|c|}
\hline & \multicolumn{4}{|c|}{$\begin{array}{l}\text { Condensing Water Flow: } 90 \mathrm{gpm} \\
\text { Chilled Water Flow: } \quad 60 \mathrm{gpm} \\
\text { Hot Water Flow: }\end{array}$} & \multicolumn{3}{|c|}{$\begin{array}{l}\text { Temperalures in } \\
\text { Degrees Fahrenhe:it }\end{array}$} \\
\hline & $\begin{array}{l}\text { Hot } \\
\text { Water } \\
\text { Inlet } \\
\text { Temp } \\
\end{array}$ & $\begin{array}{l}\text { Hot } \\
\text { Water } \\
\text { Oullet } \\
\text { Temp. }\end{array}$ & $\begin{array}{l}\because \\
\text { Energy } \\
\text { Inpul } \\
\text { Blu'h } \\
\end{array}$ & $\begin{array}{l}\text { Leaving } \\
\text { Chilled } \\
\text { Water } \\
\text { Temp } \\
\end{array}$ & $\begin{array}{r}\text { DELIV } \\
\text { CAPA } \\
\text { Btu/h } \\
\end{array}$ & $\begin{array}{l}\text { RED } \\
\text { IIY }\end{array}$ & $\begin{array}{c}\text { Heat } \\
\text { lo be } \\
\text { Rejected } \\
\text { Btiinh } \\
\end{array}$ \\
\hline & 160 & $\begin{array}{l}157.0 \\
1566 \\
1560\end{array}$ & $\begin{array}{l}132,000 \\
149.200 \\
175.900\end{array}$ & $\begin{array}{l}40 \\
45 \\
50\end{array}$ & $\begin{array}{r}66.000 \\
88.800 \\
102,000\end{array}$ & $\begin{array}{l}5.5 \\
74 \\
85\end{array}$ & $\begin{array}{l}198.000 \\
238.000 \\
277.900\end{array}$ \\
\hline \multirow{8}{*}{ 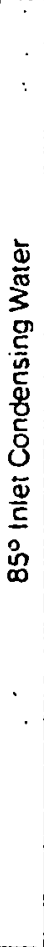 } & 165 & $\begin{array}{l}161.1 \\
1605 \\
159.9\end{array}$ & $\begin{array}{l}172.600 \\
197.900 \\
227.600\end{array}$ & $\begin{array}{r}\because 40 \\
\because \quad 45 \\
50\end{array}$ & $\begin{array}{r}98.400 \\
129.600 \\
145.000\end{array}$ & $\begin{array}{r}8.2 \\
10.8 \\
12.1\end{array}$ & $\begin{array}{l}271,000 \\
347.500 \\
372,600\end{array}$ \\
\hline & 170 & $\begin{array}{l}165.1 \\
164.5 \\
1640\end{array}$ & $\begin{array}{l}214.300 \\
240.700 \\
262.400\end{array}$ & $\begin{array}{l}40 \\
45 \\
50\end{array}$ & $\begin{array}{l}132.000 \\
166.800 \\
180.000\end{array}$ & $\begin{array}{l}11.0 \\
13.9 \\
15.0\end{array}$ & $\begin{array}{l}346.300 \\
407.500 \\
442.400\end{array}$ \\
\hline & 175 & $\begin{array}{l}169.1 \\
1687 \\
168.2 \\
\end{array}$ & $\begin{array}{l}257.000 \\
277.600 \\
297.500\end{array}$ & $\begin{array}{l}40 \\
45 \\
50\end{array}$ & $\begin{array}{l}163.200 \\
196.800 \\
213.600\end{array}$ & $\begin{array}{l}136 \\
164 \\
178 \\
\end{array}$ & $\begin{array}{l}420,200 \\
474.400 \\
511.100\end{array}$ \\
\hline & 180 & $\begin{array}{l}173.1 \\
172.9 \\
1722 \\
\end{array}$ & $\begin{array}{l}302.400 \\
313.800 \\
341.200 \\
\end{array}$ & $\begin{array}{r}40 \\
45 \\
50 \\
\end{array}$ & $\begin{array}{l}192.000 \\
224.400 \\
246.000\end{array}$ & $\begin{array}{l}160 \\
187 \\
2155 \\
\end{array}$ & $\begin{array}{l}494.400 \\
538.200 \\
587.200\end{array}$ \\
\hline & 185 & $\begin{array}{l}1769 \\
1768 \\
1763 \\
\end{array}$ & $\begin{array}{l}352,300 \\
354,900 \\
380,700\end{array}$ & $\begin{array}{l}40 \\
45 \\
50\end{array}$ & $\begin{array}{l}218.400 \\
252.000 \\
276.000 \\
\end{array}$ & $\begin{array}{l}18.2 \\
21.0 \\
23.0\end{array}$ & $\begin{array}{l}570.700 \\
606.900 \\
656.700\end{array}$ \\
\hline & 190 & $\begin{array}{l}1809 \\
180.8 \\
180.4 \\
\end{array}$ & $\begin{array}{l}396.000 \\
400,000 \\
421.500\end{array}$ & $\begin{array}{r}40 \\
\therefore \quad 45 \\
\quad 50 \\
\end{array}$ & $\begin{array}{l}237.600 \\
276,000 \\
306,000\end{array}$ & $\begin{array}{l}19.8 \\
23.4 \\
25.5\end{array}$ & $\begin{array}{l}633.600 \\
676.000 \\
727.500\end{array}$ \\
\hline & 195 & $\begin{array}{l}184.7 \\
184.8 \\
184.6 \\
\end{array}$ & $\begin{array}{l}448.700 \\
446.700 \\
451,600 \\
\end{array}$ & $\begin{array}{r}\therefore \quad 10 \\
4 ! \\
50 \\
\end{array}$ & $\begin{array}{l}2.58 .000 \\
306.000 \\
328.800 \\
\end{array}$ & $\begin{array}{l}21.5 \\
25.5 \\
274 \\
\end{array}$ & $\begin{array}{l}706.700 \\
752.700 \\
780.400 \\
\end{array}$ \\
\hline & 200 & $\begin{array}{l}188.7 \\
1890 \\
1886 \\
\end{array}$ & $\begin{array}{l}490,900 \\
481.800 \\
493.200\end{array}$ & $\begin{array}{l}40 \\
45 \\
50 \\
\end{array}$ & $\begin{array}{l}270.000 \\
318.000 \\
360.000 \\
\end{array}$ & $\begin{array}{l}22.5 \\
265 \\
300 \\
\end{array}$ & $\begin{array}{l}760.900 \\
799.800 \\
853.200\end{array}$ \\
\hline \multirow{8}{*}{ 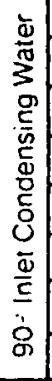 } & 165 & 1628 & 96.800 & 45 & 60.000 & 50 & 156.800 \\
\hline & 170 & 1669 & 139.100 & 45 & 96.000 & 80 & 235.100 \\
\hline & 175 & 1707 & 188.000 & 45 & 134.000 & 11.2 & 322.400 \\
\hline & 180 & 1747 & 234.000 & 45 & 168.000 & 140 & 402.000 \\
\hline & 185 & 1787 & 274.000 & 45 & 195.600 & 163 & 469.600 \\
\hline & 190 & 1825 & 327.900 & 45 & 225600 & 188 & 553.400 \\
\hline & 195 & 1866 & 365.500 & 45 & 237.600 & 198 & 603.100 \\
\hline & 200 & 1904 & 414.500 & 415 & 252.000 & 2.10 & 606.500 \\
\hline
\end{tabular}

\begin{tabular}{|c|c|c|c|c|c|c|c|}
\hline & \multicolumn{4}{|c|}{$\begin{array}{ll}\text { Concisensing Wilte: Flow } 90 \mathrm{gpm} \\
\text { Chillod Water Flow. } & 60 \mathrm{gpm} \\
\text { Hot Water Flow: } & 90 \mathrm{gpm}\end{array}$} & \multicolumn{3}{|c|}{$\begin{array}{l}\text { Temperaluies in } \\
\text { Desprees Faturenheil }\end{array}$} \\
\hline & $\begin{array}{l}\text { Hot } \\
\text { Water } \\
\text { lnlet } \\
\text { Temp. }\end{array}$ & $\begin{array}{l}\text { Hot } \\
\text { Water } \\
\text { Outlet } \\
\text { Temil) } \\
\end{array}$ & $\begin{array}{c}\text { Energy } \\
\text { Input } \\
\text { Btuin }\end{array}$ & $\begin{array}{l}\text { Leaviny } \\
\text { Chilled } \\
\text { Water } \\
\text { Telnp. }\end{array}$ & $\begin{array}{r}\text { DELIV } \\
\text { CAPA } \\
\text { BUW/1Y } \\
\end{array}$ & $\begin{array}{l}E D \\
Y\end{array}$ & $\begin{array}{c}\text { Heat } \\
\text { to be } \\
\text { Rejected } \\
\text { Blu'h }\end{array}$ \\
\hline \multirow{9}{*}{ 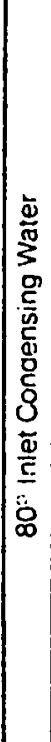 } & $\begin{array}{l}160 \\
160 \\
\end{array}$ & $\begin{array}{l}156.1 \\
1559 \\
\end{array}$ & $\begin{array}{l}174.200 \\
180.000 \\
\end{array}$ & $\begin{array}{l}40 \\
45\end{array}$ & $\begin{array}{l}108,000 \\
115.200 \\
\end{array}$ & $\begin{array}{l}90 \\
9.6\end{array}$ & $\begin{array}{l}282.200 \\
295.200 \\
\end{array}$ \\
\hline & $\begin{array}{l}165 \\
165 \\
\end{array}$ & $\begin{array}{l}160.3 \\
159.9 \\
\end{array}$ & $\begin{array}{l}210.000 \\
227.500 \\
\end{array}$ & $\begin{array}{l}40 \\
45\end{array}$ & $\begin{array}{l}1: 18.000 \\
152.400 \\
\end{array}$ & $\begin{array}{l}115 \\
127 \\
\end{array}$ & $\begin{array}{l}348.000 \\
379.900 \\
\end{array}$ \\
\hline & $\begin{array}{l}170 \\
170\end{array}$ & $\begin{array}{l}164.1 \\
163.8\end{array}$ & $\begin{array}{l}259.000 \\
270.900\end{array}$ & $\begin{array}{l}40 \\
45\end{array}$ & $\begin{array}{l}174,000 \\
189,600\end{array}$ & $\begin{array}{l}145 \\
158\end{array}$ & $\begin{array}{l}432.900 \\
.110 .500\end{array}$ \\
\hline & $\begin{array}{l}175 \\
175 \\
\end{array}$ & $\begin{array}{l}168.0 \\
167.7 \\
\end{array}$ & $\begin{array}{l}306,700 \\
321.100 \\
\end{array}$ & $\begin{array}{l}40 \\
45 \\
\end{array}$ & $\begin{array}{l}206.400 \\
228,000 \\
\end{array}$ & $\begin{array}{l}17.2 \\
190 \\
\end{array}$ & $\begin{array}{l}513.100 \\
549.100 \\
\end{array}$ \\
\hline & $\begin{array}{l}180 \\
180 \\
\end{array}$ & $\begin{array}{l}172.0 \\
171.8\end{array}$ & $\begin{array}{l}351,800 \\
357,500 \\
\end{array}$ & $\begin{array}{l}40 \\
45\end{array}$ & $\begin{array}{l}236.400 \\
259.200 \\
\end{array}$ & $\begin{array}{l}19.7 \\
21.6 \\
\end{array}$ & $\begin{array}{l}588.200 \\
616.700 \\
\end{array}$ \\
\hline & $\begin{array}{l}185 \\
185 \\
\end{array}$ & $\begin{array}{l}176.0 \\
175.7 \\
\end{array}$ & $\begin{array}{l}395.700 \\
405.000 \\
\end{array}$ & $\begin{array}{l}40 \\
45\end{array}$ & $\begin{array}{l}260.400 \\
291.600 \\
\end{array}$ & $\begin{array}{l}21.7 \\
243 \\
\end{array}$ & $\begin{array}{l}656.100 \\
696.600 \\
\end{array}$ \\
\hline & $\begin{array}{l}190 \\
190 \\
\end{array}$ & $\begin{array}{l}179.8 \\
179.7\end{array}$ & $\begin{array}{l}446.200 \\
449.800\end{array}$ & $\begin{array}{l}40 \\
15\end{array}$ & $\begin{array}{l}282.000 \\
321.600\end{array}$ & $\begin{array}{l}235 \\
26.8\end{array}$ & $\begin{array}{l}728.200 \\
771.400\end{array}$ \\
\hline & $\begin{array}{l}195 \\
195 \\
\end{array}$ & $\begin{array}{l}1836 \\
1837 \\
\end{array}$ & $\begin{array}{l}500.000 \\
492.000 \\
\end{array}$ & $\begin{array}{l}40 \\
45 \\
\end{array}$ & $\begin{array}{l}300.000 \\
344.400 \\
\end{array}$ & $\begin{array}{l}25.0 \\
28.7 \\
\end{array}$ & $\begin{array}{l}800.000 \\
836.400 \\
\end{array}$ \\
\hline & $\begin{array}{l}200 \\
200\end{array}$ & $\begin{array}{r}187.3 \\
1878\end{array}$ & $\begin{array}{l}547.400 \\
527.900\end{array}$ & $\begin{array}{l}40 \\
45\end{array}$ & $\begin{array}{l}312.000 \\
360.000\end{array}$ & $\begin{array}{l}26.0 \\
300\end{array}$ & $\begin{array}{l}859.400 \\
887.900\end{array}$ \\
\hline
\end{tabular}

PRE SSURE DRIOP for P(Im) Sizuly

In Feel of Watter at Flow Rate. Gallons pet Minule

\begin{tabular}{|c|c|c|c|c|c|c|c|c|c|}
\hline 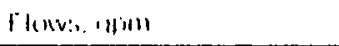 & $: 3()$ & .111 & 1,0 & (i) & $\therefore$ & $M u$ & $! 11)$ & $1(x)$ & 1111 \\
\hline Hen Whate Circoun & isn & MI & fiti & 9.1 & $1: r$ & $16 i$ & $? i$ & 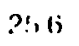 & NA \\
\hline 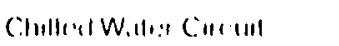 & $? !$ & .1 .1 & 68 & 98 & $1: 3.1$ & $1 i 1$ & $: 118$ & 269 & NA \\
\hline Condensmen Wollt Cucint & NA & NA & in & $10 !$ & 1.11 & $18: 3$ & $: 39$ & $2 i n$ & $33 !$ \\
\hline
\end{tabular}

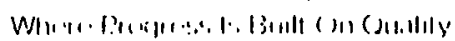

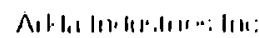

P() L in s s.s.

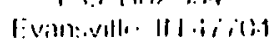

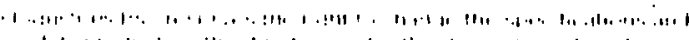

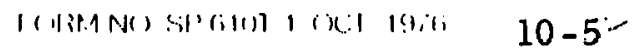

minงtน") 
Proper maintenance is a necessity to insure continous, efficient operation of the equipment. It prolongs the 1 ife of the equipment and reduces service requirements.

The maintenance requirements on Arkla's Solaire Units are relatively simple. The suggested monthly routine can be done by competent building maintenance personnel.

Spring and fall change-overs and any service should be handied by trained servicemen.

Maintenance on auxiliary equipment to the Arkla unit should be performed according to the manufacturer's recommendations.

In the suggested inspection routines, reference is made to page numbers in the Arkla Service Manual. This is to aid in finding the information needed on a particular subject.

As these inspections are made, all temperatures and adjustments should be recorded. Changes in temperature or conditions should be noted for discussion with the servicing agency.

\section{MONTHLY INSPECTIONS}

COOLING:

1. Take a complete, set of temperature readings $(V \mid-15)$. If a problem is indicated, call servicing agency.

2. Check cooling tower;

a. Cleanliness of sump.

b. Cleanliness of sump screen.

c. Condition of fan belt.

d. Check water distribution system.

3. Check condensing water bleed-off flow rate (IV-35-2)

4. Check operation of condensing water chemical treatment equipment (if using).

5. Open all valves, on dirt legs and strainers, long enough to flush out any dirt or trash.

6. Visually check piping for leaks.

7. Perform maintenance on auxiliary equipment as per manufacturer's instructions.

8. Check equipment and area for cleanliness.

SPRING START-UP

A. TOWER

1. Clean and flush distribution system and sump and sump strainer. 
5. Check operation of chilled water low temperature swilch. $(V-12-4)$

6. Check operation of flow switch. $(V-15-1)$

7. Check operation of evaporator low temp switch. $(V-11-3)$

8. Check hot water valve modulation. (IV-43-10)

9. Check all external controls in the system.

D. OPERATIONAL CHECK

1. Place thermometers in all thermometer wells $(V|-| 5-6(A),(B),(C))$.

2. Operate unit on cooling for at least 30 minutes or until all temperatures have stabilized.

3. Record temperatures.

\section{SHIUT DOWN}

A. TOWER

NOTE: If the condensing water system could be subjected to sub-freezing temperatures it is recommended that the system be flushed with a mixture of anti-freeze and water after performing the shut down procedure given below. This mixture should be capable of withstanding the lowest expected ambient temperature.

1. Close valve in tower make-up water line.

2. Open all drain valves and remove all plugs in condensing water system.

3. Clean and flush tower's distribution system.

4. Clean and flush tower sump.

5. Clean all strainers in condensing water systern.

6. Circulate anti-freeze through condensing water circuit.

7. Remove fuses so that condensing water pump or tower fan cannot accidentally be operated without water.

B. UNIT

1. Turn off unit.

2. Open all valves to drain the condensing water circuit.

3. Turn off manual hot water supply valve to the unit. Open drain valves.

4. Check anti-freeze concentration (IV-29-2) and close chilled water valves at unit.

5. Touch up all rusty areas on unit by painting. Arkla Part No. Z-3181 Pizzaz (Orange Paint), or Gliddens Poly Urethane Floor Enamel, Color No. 15157(Tinted). 


\section{MARLEY}

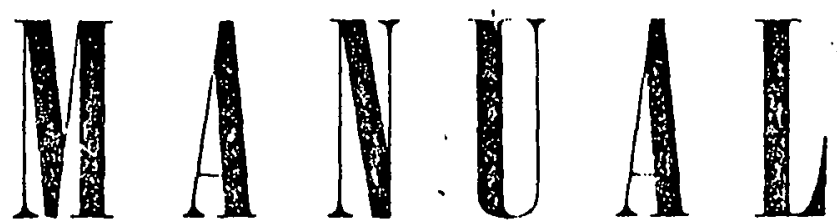

\section{Installation, Operation}

and

Maintenance Instructions

SERIES 4600

STEEL AQUATOWERS 


\title{
Installation, Operation and Maintenance Instructions
}

\author{
SER I ES. 4600
}

\section{STEEL AQUATOWERS}

\section{TOWER LOCATION}

Locate so prevailing wind will blow into the louvered face. Direct fan discharge away from building surfaces to eliminate the possibility of discoloration. Locate so there is free flow of air to and from the tower. Allow clearance on all sides for maintenance. Anchor in a level position to a stable foundation.

\section{INDOOR INSTALLATION}

A duct is required from the tower air discharge to the outside. In some cases it may also be desirable to install an inlet air duct. If ducts are used, the total draft loss should not exceed .10" water pressure. Uraft losses can be minimized by:

a. Using $20 \%$ oversize ducts.

b. Avoiding sharp turns or abrupt changes in size.

c. Keeping duct length to a minimum.

d. Increasing the area of screened or louvered opening so. the net free area is at least $20 \%$ greater than the tower discharge opening area.

Ducts should be attached to the tower using rubber or canvas connections. Access openings for servicing the mechanical equipment must be provided if air discharge ducts are installed. If the duct discharges into the prevailing wind, it may be necessary to install a windbreak or an elbow to serve as a deflector. Ducts installed on towers with year around usage should be water tight and insulated to prevent condensation.

\section{TOWER INSTALLATION}

The tower is shipped complete with the motor in a carton and miscellaneous parts package within the tower. Anchor tower by bolting a clip to the foundation and basin sides or through slots in legs using $3 / 8^{\prime \prime}$ or larger bolts.

PIPING TO TOWER (Summer Temperature Conditions)

1. Use pipes of sufficient size to provide minimum friction loss.

2. Connect Float Valve to make-up water supply.

3. Install bleed-off line. Bleed-oft is the continuous wasting of a sinall amount of water during operation which retards scale and corrosion. A bleed-oft line can bc installed at any point in the system, however, the best point is in the hot water line near the top of the tower so water will be removed when the puinp is operating. A cojper tube, pinched down or with a pet cuck can be used.

PIPING TO TOWER (Winter Freezing Conditions)

1. Where operating conditions require tower use during freezing weather, it is recommended that the towers be installed for "dry basin" operation. See Figure 1.

2. Provide an inside open-type storage tank with a capacity of four times the cooling tower GPM.

3. Connect tower suction to storage tank.

4. Install make-up water, bleed-off, overflow and drain lines on tank.

5. Insulate and heat watcr lines exposed to freezing.temperatures. 


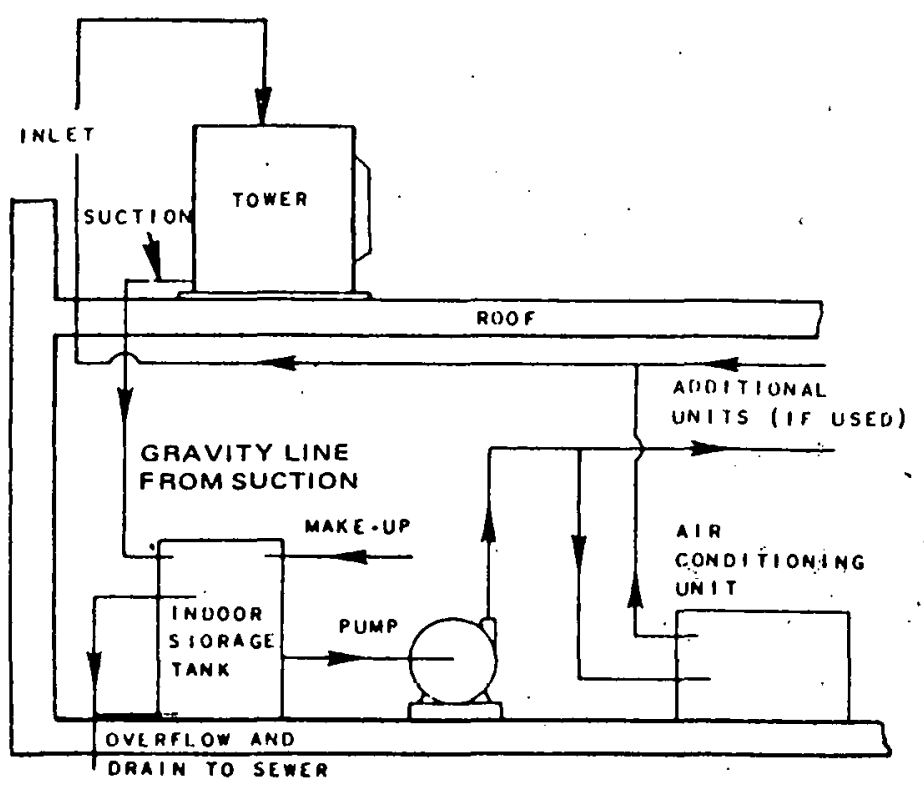

FIGURE 1. Flow Diagram for a Cooling Tower with Indoor Storage Tank. (Piping Is Arranged So That Tower Basın Will Drain When Pump /s Shut Off.)

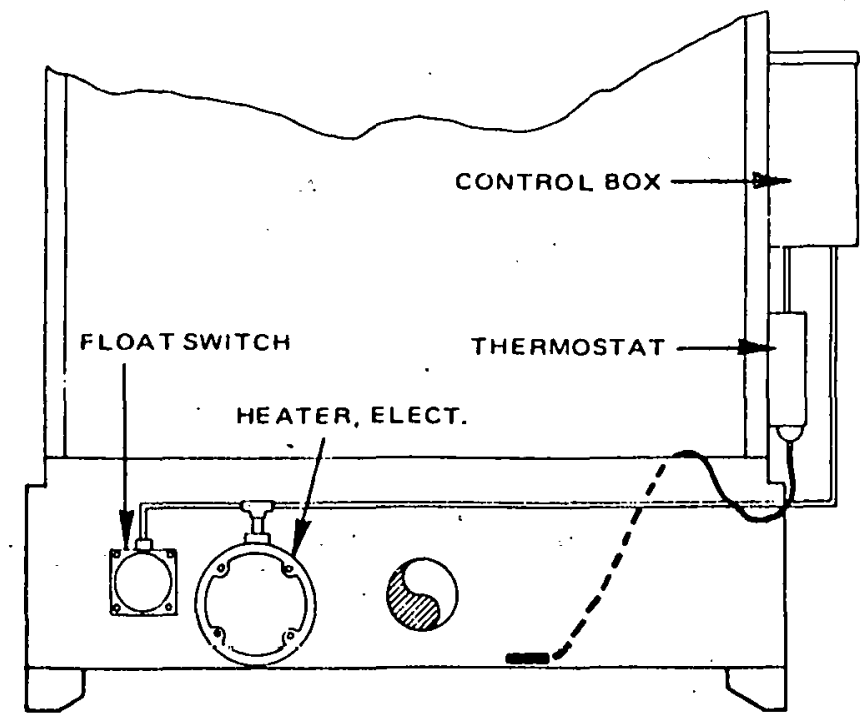

FIGURE 2. Electric heater systems are available as optional eg̈ulpment to prevent freeziny l/e cold water basin when it cannot be drained.

\section{MECHANICAL EQUIPMENT INSTALLATION}

\section{MOTOR, SHEAVE AND V-BELT INSTALLATION}

1. Check the motor name plate to be sure its voltage, phase and frequency ratings are the same as the power supply.

2. Check to insure that fan is tightly secured to bearing housing shaft and free to rotate and that bearing housing is secure to support.

3. Install all thread belt tension adjusting bolts in motor base cradle, see Detail A. Install lock waslicis and nuts, fạstening adjusting bolts to inotor base cratle. Run galvanized nuts about halfway down on bolts. Insert bolts through slots in motor base, install lock washers and run top nuts down, locking base in place. Bolt motor to motor base.

4. Install motor sheave and align it with fan sheave. A plumb line will be helpful in aligning sheaves. See Detail "B".

5. Install V-belt and adjust tension by means of belt tension adjusting bolts. A correctly tensioned bett does not slip when the fan is started; and, when running, the "tight" side is straight between sheaves. The "slack" side will have a slight how. Correct tension-can only be determined by trial runs at successively higher tensions until slipping has stopped.

A small further increase in tellsion should be made to account for norinal belt stretch. Avoid over tensioning. Too much tension reduces bearing and belt life.
New belts must be retensioned after 8 to 12 hours operation since nuw belts stretch at a higher rate and "seat" into sheave grooves.

6. Connect motor to power supply using wiring, switching, short circuit protection and overload protection in accordance with the National Electric Code and local requirements. Failure to wire the motor correctly will void its warranty. The overload protection for Motors must be part of the control system, see Diagram " $C$ ".

7. Sleeve bearing motors are usually shipped without oil and niust he oiled before operating. Ball bearing motors are lubricated for the initial operatiun liy the manufacturer.

8. Install belt guard using sheet mntal screws. See Figure i (paye 8).

\section{INSTALLATION OF OPTIONAL EQUIPMENT}

\section{AIR INLET SCREEN}

Install hardware used to attach air inlet screen to side cas. ing sheets. Set screen in position and install with wing nuts.

\section{HOT WATER BASIN COVER}

Install "S" strins at hot water basin sides. Remove from splash box cover those sheet metal screws indicated on installation drawing. Position basin cover sugnents and reinstall sheet metal screws. 


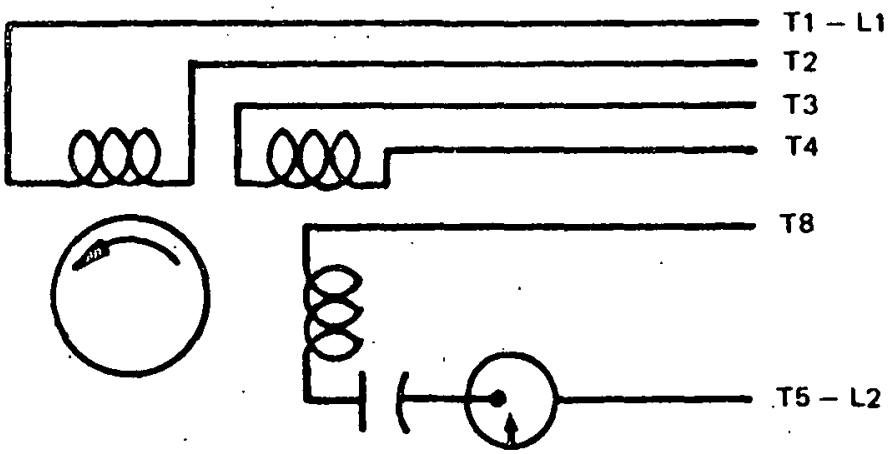

Without Thermal Overload (Integral HP)

HIGH VOLTAGE

1. Connect $T I$ and $L 1$ and insulate.

2. Connect T2, T3 and T8 and insulate

3. Connect T4 and T5 and insulate.

LOW VOLTAGE

1. Connect T1, T3, T8 and $L 1$ and insulate.

2. Connect T2, T4, T5 and L2 and insulate.

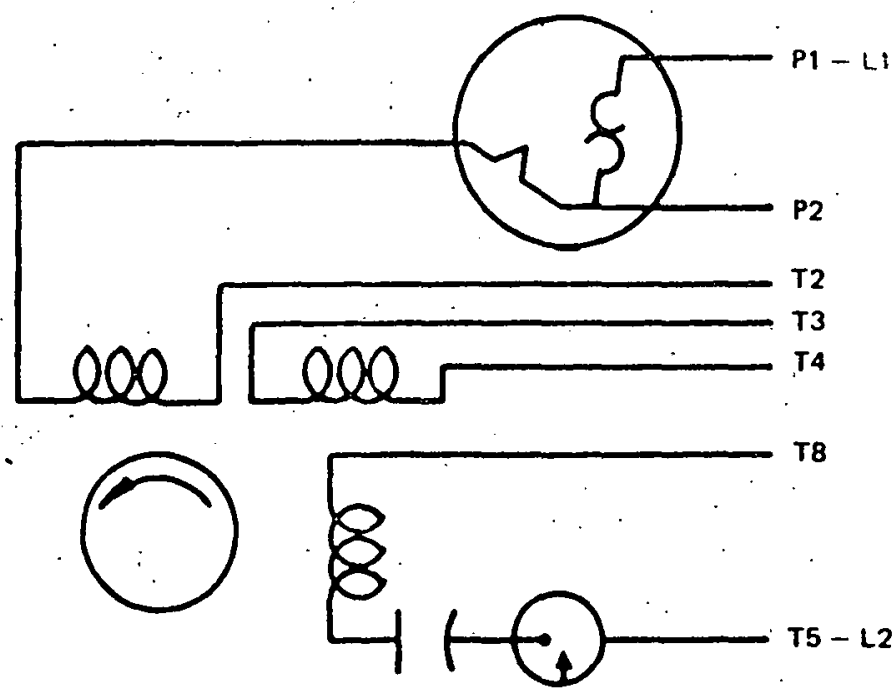

With Thermal Overload (Fractional HP)

HIGH VOLTAGE

1. Insulate $P 2$.

2. Connect T2, T3 and T8 and insulate.

3. Connect T4, T5 and L2 and insulate.

4. Connect $P I$ and $L 1$ and insulate.

NOTE: Colors may be substituted for numbers as follows:

\section{LOW VOLTAGE}

1. Connect $P 1$ and $L 1$ and insulate.

2. Connect $P 2$ and $T 3$ and insulate.

$\begin{array}{ll}\text { T1 - Blue } & \text { T5 - Black } \\ \text { T2 - White } & \text { T8 - Red } \\ \text { T3 - Green } & \text { P1 - No Color Assigned } \\ \text { T4 - Yellow } & \text { P2 - Brown }\end{array}$

3. Connect $\mathrm{T} 1$ and $\mathrm{T} 8$ and insulate.

4. Connect T2, T4 and T5 and insulate.

To reverse rotation, interchange leads $T 5$ and $T 8$.

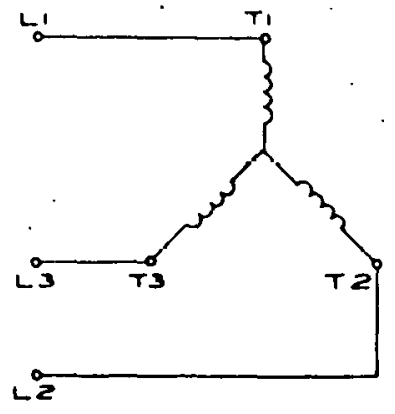

ReO VQLT

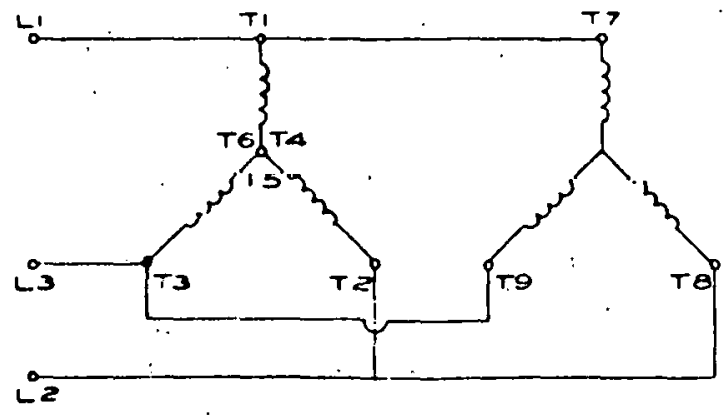

$230 \vee O L T$

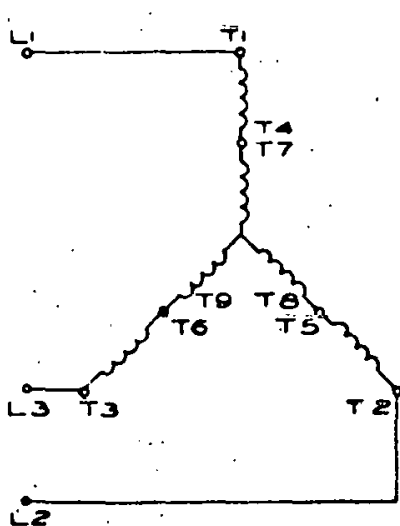

$460 \vee$ ㅇㄴ $T$

WIRING DIAGRAM 3 PHASE MOTORS 


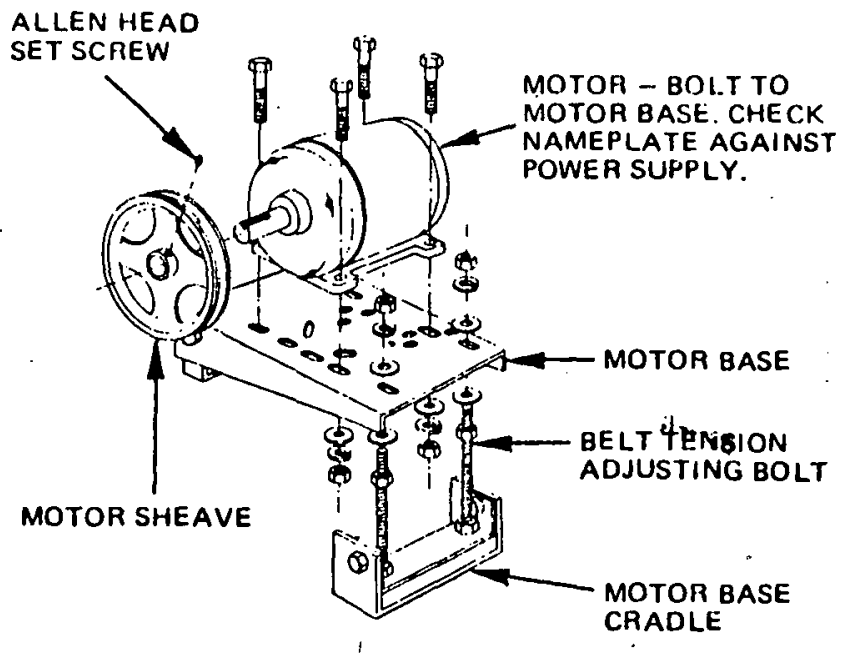

DETAIL "A"

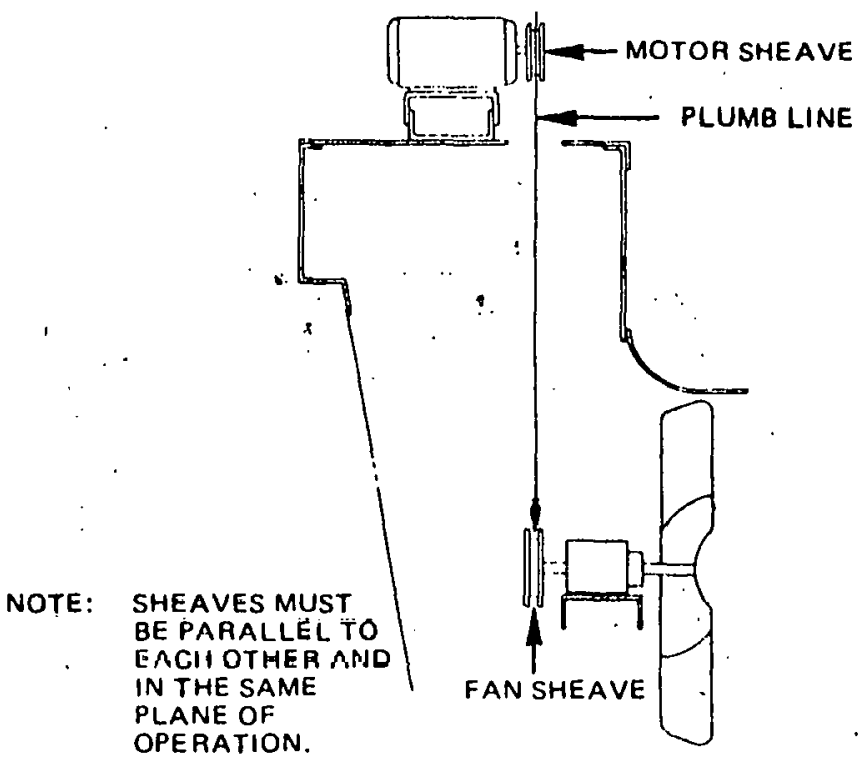

DETAIL "B"

\section{OPERATION INSTRUCTIONS}

1. Wash foreign matter from fill and basin.

2. Fill circulating system with water.

3. Start pump and adjust float valve to maintain 4 " 15 " on models 4619 thril 4625 and $8^{\prime \prime}$ on modets 4627 thru 4633) of water in cold water basin.

4. Check bleed-off line to make sure water is being discharged during operation.

5. Check fan for free rotation and oil level in Bearing Housing (see maintenance instructions). Start motor and check direction of rotation. Fail must rotate clockwise when viewed from the tan discharge side. If the rotation

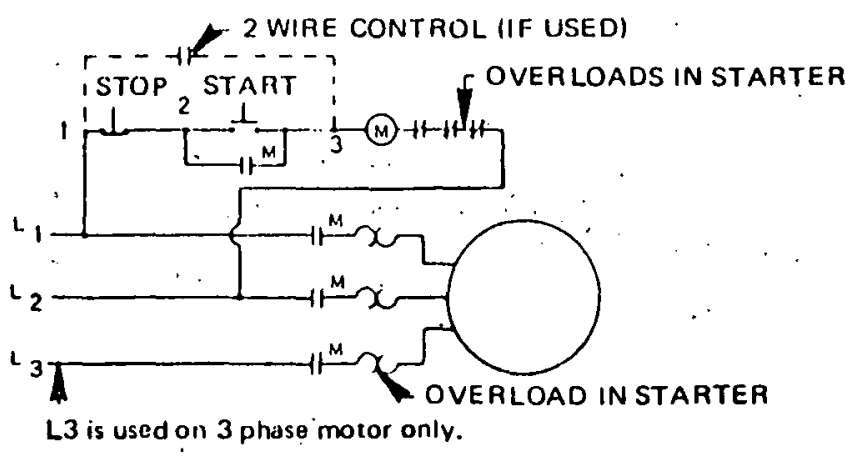

Showing Overload in Starter DINGRAM "C"

is incorrect, change any two of the thice motor leads for a three phase motor or interchange the connections of either the main or starter windings for single-phase capacitor start motor.

6. Depth of water in hot water basin struld be uniform. If the basin overflows, reduce the flow rate. Do not pump $\because$ more water than design capacity.

7. Do not cycle the motor so that the total of the starting times exceeds 30 seconds each hour.

?

\section{MAINTENANCE INSTRUCTIONS}

\section{MOTOR LUBRICATION}

Lubricate the motor accordina to the motor manufacturer's instructions shipped with the motor. Motor should be relubricated at the start and end of each operating season.

\section{FAN BEARING LUBRICATION}

Lubriçte bearing housings with SAE 20 mineral oil.

Oil cups should be kept full to insure proper oil level in bearing housings.

\section{BEL.T TENSION}

Chcck belt tonsion every two to thr we weeks during peak operating season. Refer to page 4 , item 5.

\section{BLEED -OFF}

Check the bleed-off for continuous water discharge.

\section{BASIN AND SUCTION SCREEN}

Drain and clean cold water basin and suction screen perlodically.

\section{FLOAT VALVE}

Cluck flodt valve periodically for proper operation and maintenance of water level. 


\section{GENERAL}

The following tables show the proper amount of bleed-off.

\section{COOLING RANGE:; DEGREES F}

\begin{tabular}{l}
6 \\
$7-1 / 2$ \\
10 \\
15 \\
20 \\
\hline
\end{tabular}

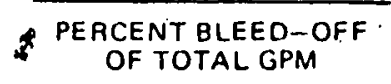

.15

.22

.33

.54

.75

\section{CHEMICAL TREATMENT}

The dissolved solids in the circulating water are concentrated by evaporation and must be limited by bleed-off of some of the water. Chemical treatment is not normally required if adequate bleed-off is maintained.

Algae and slime may occur and can be controlled by care. ful application of proper chemicals. Improper application of concentrated water treating chemicals may damage parts of the system. If scale or algae and slime accumulate, obtain the services of a competent witer treating consultant.

\section{WATER DISCOLORATION}

Discoloration of water may ockur when a cooling tower is placed in operation. This disculoration is not harmful and will normally disappear after several, weeks of operatinn. Application of a common bleach solut or ivitifiased bleed-off will alleviate this condition.

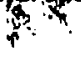

\section{FOAMING}

Foaming may occur when the concentration of dissolved solids in the circulating water is high. Increasing the bleedoff rate or application of commercial foam depressants will alleviate the problem. Foam dopressants slightly alter the physical properties of the circulating water and may affect the tower operating characteristics. Dosages should not exceed manufacturer's recommended amounts. Trial of several brands may be necessary to achieve optimum foam control and tower operation.

\section{SEASONAL SHUTDOWN INSTRUCTIONS}

\section{BASIN AND FRAME}

Drain the tower basins and all exposed piping. Leave the basin drain open. Water may be left in cold water basin if tower is located in a non-freezing area.

During shutdown, clean the tower and make any necessary repairs. Apply protective coating as required to all metal parts. Particular attention shuuld be given to bearing supports.

\section{MECHANICAL EQUIPMENT}

\section{V.BELTS AND SHEAVES}

1. At shutdown, remove and store belts in a cool, dark, $f$ dry room. Clean and coat, sheave grooves with rust pre. ventive, lacquer, or naint.

2. Before putting belts back on sheaves, remove rust preventive. Replace belts with excessive wear.

3. When putting tower back into service refer to "Mechanical Equipment Installation" page 4 for belt installation and tensionıny instructions.

BEARING HOUSING;'Oil Lubricated Type

1. At shutdown, operate until oil is warm; drain and refill. Use SAE 20 mineral oil.

2. Each month, drain water condensate at the drain plug. Check oil level and add oil if necessary.

9. At start-up, operate until oil is warm; drain and refill.

\section{ELECTRIC MOTORS}

Do not start motor without determining that fan is free to rotate.

Refer to motor manufacturer's recommendations for lubri. cation and maintenance instructions.

If shutdown period is longer than seasonal, contact The Marley sales office or representative in your area for additional information. Always refer to tower serial number when writing for information or ordering parts. The serial number is stamped on the tower name plate.

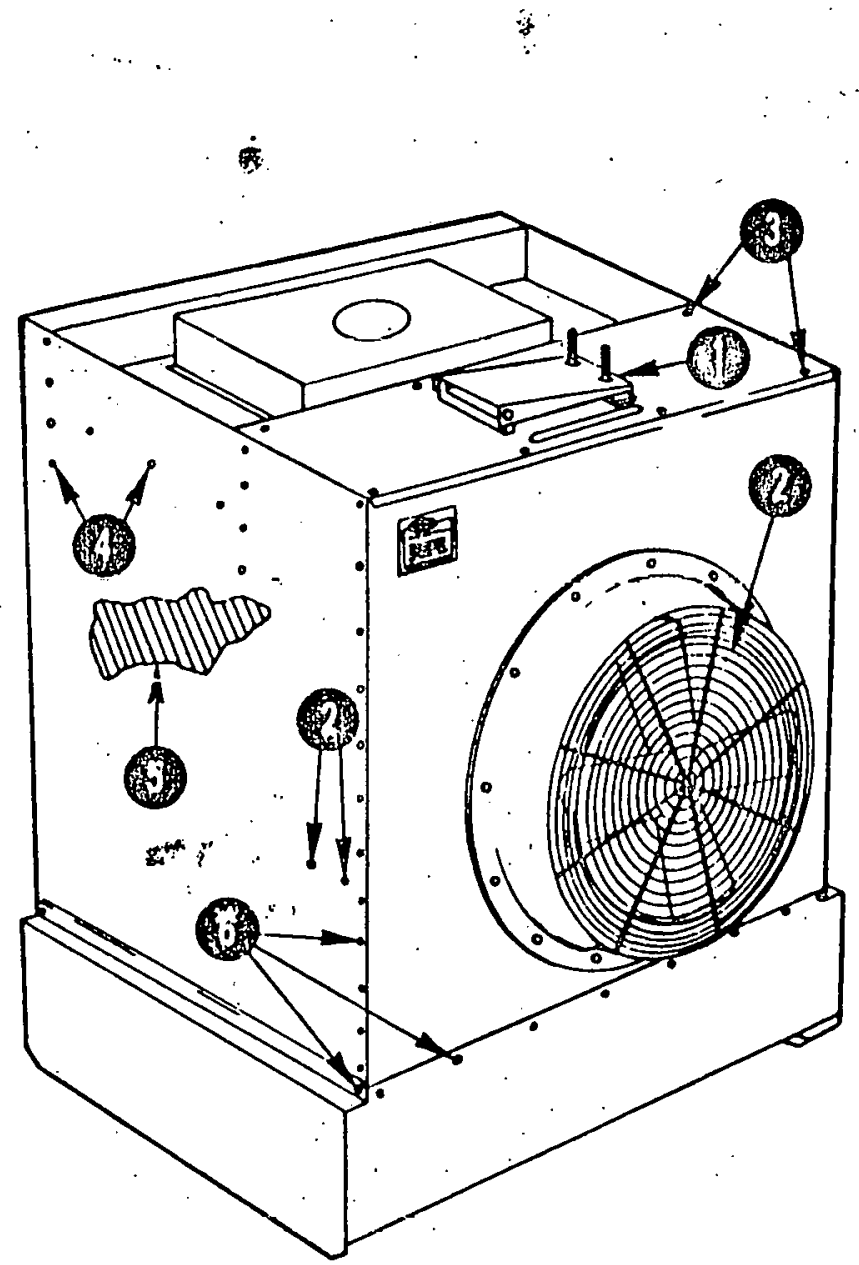




\section{DISASSEMBLY AND REASSEMBLY INSTRUCTIONS}

Never disassemble the Aquatower more than necessary; for example, if removal of the motor base is sufficient to get the tower to the installation site, remove only that part. When disassembling, remember how each part is screwed, bolted and set in place; fasten screws and bolts for each part with each section. Be careful not to mar galvanized coating.

DISASSEMBLY - 4619 thru 4633 (sequence is similar for 4613 thru 4617 )

1. Remove motor base.

2. Remove fan guard, fan and all hardware attaching bearing housing support channel to casing sheets.

3. Remove screws attaching top sheet, then remove sheet

4. Remove screws attaching distribution basin then remove basin.
5. Remove fill.

6. Remove screws from collection basin and side casing sheets at fan sheet. Remove sicle casing sheets and fan sheet with fan cylinder attached.

REASSEMBLY

Reassembly of the Acpuatower is the reverse of the steps noted above.

The following precautions are important:

1. Fill inust be installed level to assure full tower performance.

2. Bolts which use rubber sealing washers under head should be tightened securely to prevent leaks.

3. Be sure mechanical equipment is installed correctly and fan rotates freely.

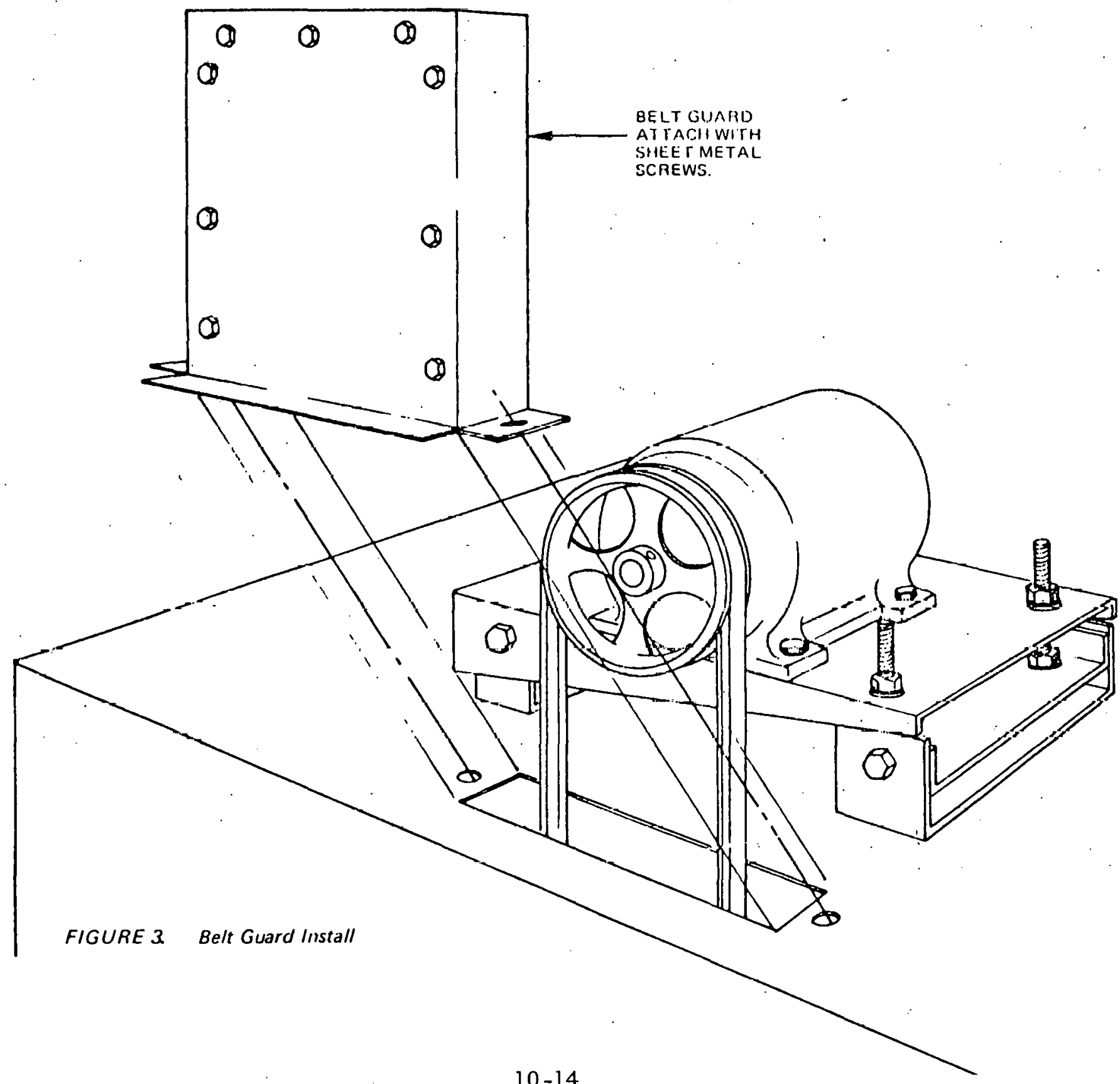




\section{LINE VOLTAGE THERMOSTATS}

\section{INSTALLATION AND \\ OPERATION INSTRUCTIONS}

SERIES T26

FORM 996-49.1

\section{Heating, Cooling, Combination Heating and Cooling}

\section{APPLICATION}

These line voltage thermostats control heating, cooling, or year 'round air conditioning units in commercial, industrial or residential installations.

Where critical or high value products are to be maintained at a specific temperature, a single thermostat should not be applied to perform as both an operating and a limit control. In these applications a separate limit control with alarm contacts should be wired to indicate when the limit control operates.

\section{LOCATION}

The thermostat should be mounted 4 to 5 feet above the floor in a location where it will be subjected to and affected by average room temperature. Do not mount thermostat where it may be affected by heat from lamps, sunlight, fireplaces, registers, radiators, pipes, etc. or by cold from windows, doors, registers, pipes, etc.

On unit heater applications, locate the thermostat below and behind the heater in the path of the air entering the unit, not in the path of the discharge air.

\section{WIRING AND MOUNTING}

All wiring should conform to the National Electrical Code and local regulations. Loads exceeding the rating of the thermostat can be handled with a relay or motor starter.

CAUTION: Disconnect power supply before wiring connections are made to prevent possible electrical shock or damage to equipment.

The thermostats are supplied with facloiy installed vertical faceplates as standard. If horizontal mounting is desired, a faceplate to convert the vertical thermostat is packed with the thermostat for on-the-job installation. If any other version is desired (concealed adjustment, less thermometer, etc.) separate faceplate kits are available for on-the-job installation.

The following procedure should be followed in the in-

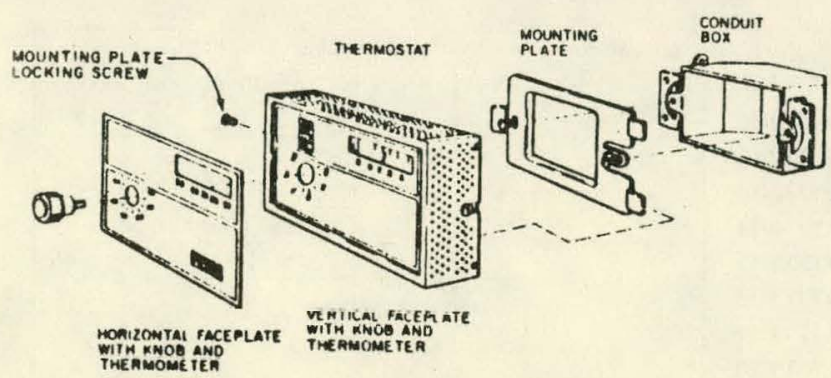

Fig. 2-line drawing illustrating method of mounting a vertical thermostat to a horizontal outlet box and installing a horizontal faceplate.

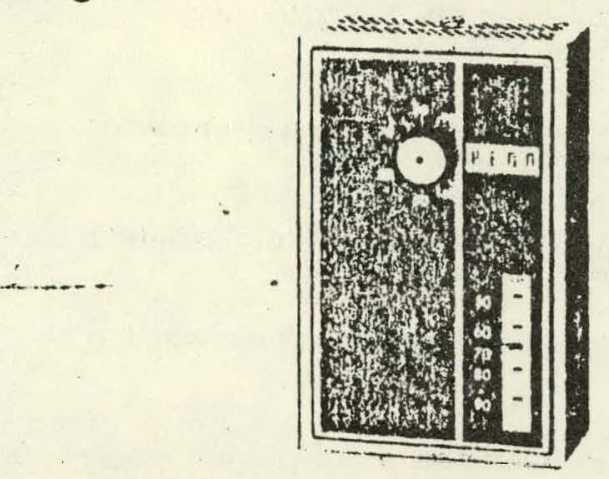

Fig. 1 - Thermostat with external adjusting knob and thermometer.

stallation. Do not remove the thermostat cover to install. All wiring and mounting can be completed without removing the cover. Thermostat terminal identification is under nounting plate.

1. Select the proper mounting location.

2. Install a $2 \times 3$ vertical or horizontal (as required) outlet box.

3. Run wire in conduit or BX to a standard $2 \times 3$ outlet box located in the wall. A standard shallow switch and receptacle box can be used where surface mounting and exposed conduit wiring are necessary, such as on a concrete or brick wall. Allow abuut 6 inches of wire for connections to thermostat terminals.

4. Remove mounting plate, see Figs. 2 and 3, and fasten plate to outlet box with screws provided in mounting plate.

5. Make the necessary wiring connections to the contact unit at the rear of the thermostat. (See Typical Application Diagrams.) Use terninal screws supplied in the contait unit. Sirews longer than $1 / 4$ " can damage the switch.

6. Hook two slots in back of thermostat over formed

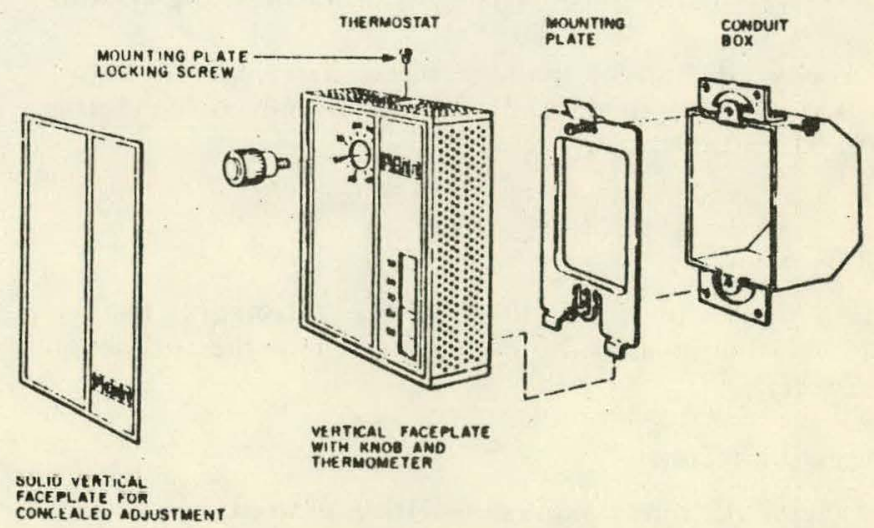

fig. 3.-Line druwing illustrating method of mounting a vertical thermostat to outlet box. Also shown is a solid vertical faceplate for concealed adjusiment when desired. 


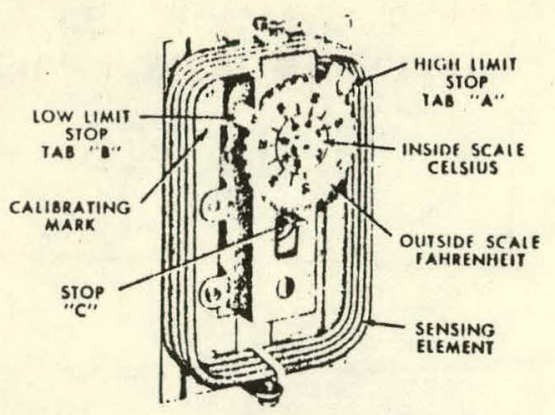

Fig. 4-Dial stops are shown above. High limit stop is sel by tab "A"; low limit stop is set by tab "B".

prongs on mounting plate and swing thermostat into position against mounting plate.

7. Tighten mounting plate locking screw, Figs. 2 and 3.

\section{Optional Faceplate Installation}

1. Mount the thermostat.

2. Ioosen screw in center of knob with a 1/16" Allen wrench and remove knob.

3. Peel off backing strip from selected faceplate.

4. Position plate over factory installed plate with one long edge and two corners aligned straight and even with installed plate.

5. Retain position and firmly press selected faceplate onto cover.

6. Replace knob, when required.

\section{ADJUSTMENTS}

Models with external knob permit thermostat adjustment by rolating knob. Indicator line on knob denotes thernostat dial setting.

For concealed dial models (with coser removed), dial setting desired should be lined up with reference mark on base of thermostat. This will put desired setting at a 9 o'clock clock-1.nc position when thermostat is held vertically. Dial settings on beries 12$) 6$ heating and SPDI thermostats indigate point at which contacts make to start heating system. Dial setting on Type T26J indicates point at which contacts make to statt cooling system.

For key adjustment, remove screw in conter of knob, adjust to set point desised and retain knob as "key" for future readjustment.

\section{LIMIT STOPS}

High limit and low limit stops are an integral feature of these thermostats. Stops may be set in the following manner:

\section{High Limit Stop}

1. Set dial to maximum stop setting desired.
2. I.cosen screw in center of knob with a $1 / 16$ " Allen wrench and renove hnob.

3. Remove thermostat cover by loosening cover screws. Remove cover.

4. While holding dial firmly in position depress tab "A," Figure 4 and rotate clockwise until tab is against stop "C.."

5. Release tab making sure tab fits into nearest notch. Notches in dial are approximately $2 \frac{1}{2^{\circ}} \mathrm{F}$ apart.

\section{Low Limit Stop}

1. Follow the same steps as outlined under "High Iimit Stop" above but rotate tab "B," Figure 4 counterclockwise.

2. Replace cover and tighten cover screws. Assemble knob. Rotate knob to desired normal operating setting.

\section{DIAL LOCK}

'The high limit stop and low limit stop can be set to prevent dial from rotating. Rotate dial to set point desired and move both tabs to a position against either side of Stop "C," as outlined in the above "High Limit" and "Low Limit" paragraphs.

\section{CALIBRATION}

The Series T26 thermostats are factory calibrated and no ficld calibration should be attempted.

\section{CHECKOUT PROCEDURE}

Before leaving installation, a complete operating cycle should be observed to see that all components are functioning properly.

\section{REPAIRS AND REPLACEMENT}

The knob. fareplate and cover are field replacealse. Oilier field repairs must not be made. Replacement thermostats may be obtained from the nearest Penn-Baso Wholesaler. When ordering a replacement thermostat, specify Produce Number and Serial Number as shown on the cover label of the thermostat.

\section{FACEPLATES}

Facrplates are available in separate hits for on-the job installation. All plates have peeloff hacking strips. liaceplates are available in all combinations shown in the following table.

\begin{tabular}{|c|c|c|c|c|c|c|}
\hline \multirow{2}{*}{$\begin{array}{c}\text { Kit } \\
\text { Number }\end{array}$} & \multicolumn{2}{|c|}{ Mnunting Position } & \multicolumn{2}{|c|}{ Type of Adiustment } & \multicolumn{2}{|c|}{ Thermomnter } \\
\hline & Vertical & Horizontal & Knob & Concealed & Yes & No \\
\hline PLT213-5 & - & $x$ & $=$ & $x$ & $=$ & $x$ \\
\hline PLT2136 & $x$ & $\therefore$ & - & $x$ & $=$ & $x$ \\
\hline PLT213-9* & $x$ & $\cdots$ & $x$ & - & $x$ & - \\
\hline PIT213 11* & - & $x$ & $x$ & - & $x$ & - \\
\hline PLT213-15 & $x$ & - & - & $x$ & $x$ & - \\
\hline PLT213-16 & - & $x$ & 一 & $x$ & $x$ & - \\
\hline PLI213 17 & $x$ & $\cdots$ & $x$ & - & - & $x$ \\
\hline PLT213.18 & $\ldots$ & $x$ & $x$ & - & - & $x$ \\
\hline
\end{tabular}

* Supplied with stanctard wholesaler models (vertical is factory installedi. 


\section{TYPICAL APPLICATION DIAGRAMS}

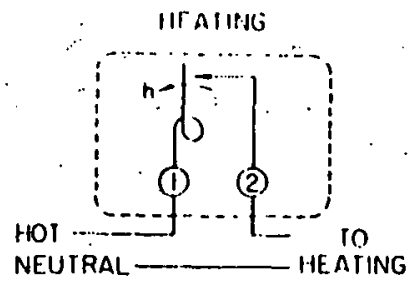

Fig. 5- Internal dirigram of Type $126 \mathrm{~A}$ and Type T26B (no seleclor).

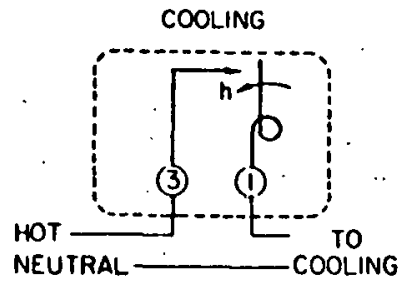

Fig. 6- Internal diogram of Type 126) (no selector).

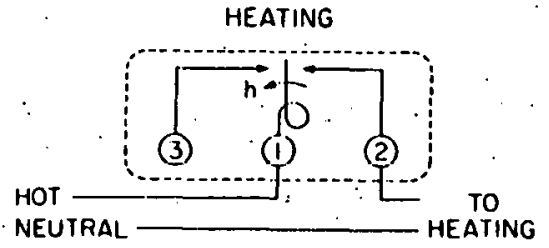

Fig. 7-Types T26S, T26T (no selector) wirod for heating. applicution.

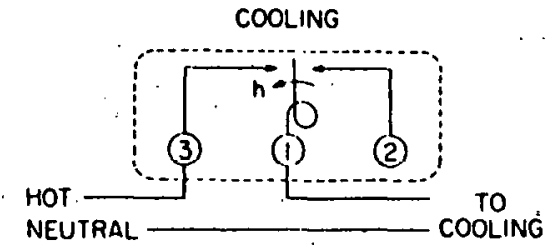

Fig. 8-Typos 1265, T26T (no selector) wired for cooling application.

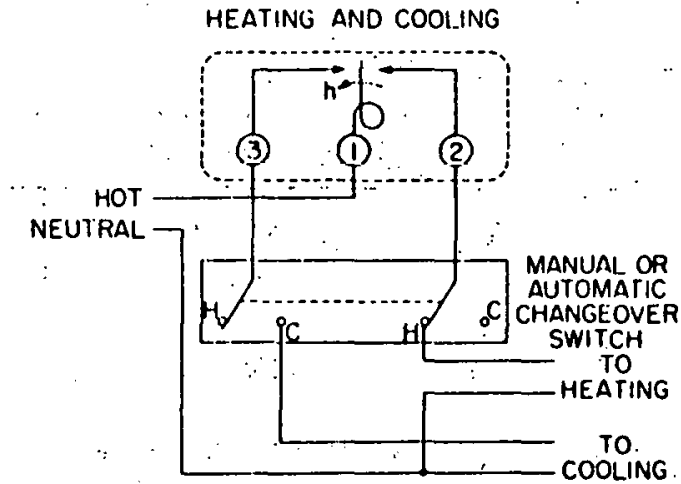

Fig. 9 - Types T26S, T26T (no soloctor) wired for healing and cooling with manual or automalic changeover switch.

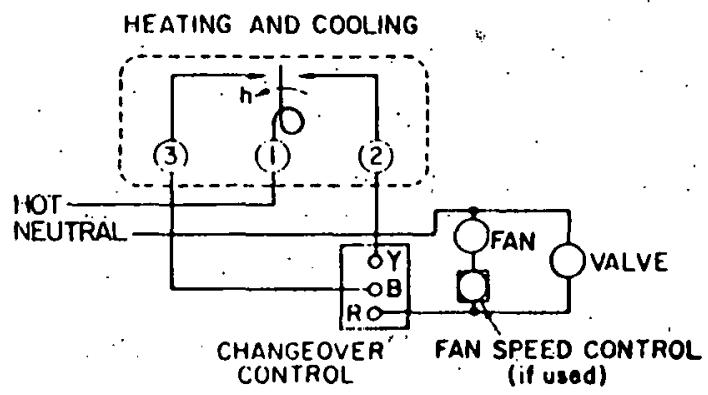

Fig. 11 - Typo T26S, T26T (no seleclor) on fon-coil unit with cycling fan and valve. Terminal markings shown for Type A19CAC ' changeover control. 


\section{REVERE \\ COPPER AND BIRASS INCORPORATED}

Buildina Paolucts Dapatmen.

P.O Hox 191

HOMH, Nuw Youk 134.11

319.338 .21111

Subjects PERFORMANCE CURVES FOR REVERE "SUN-AID" MODULAR SOLAR COLLECTORS

The attached plots demonstrate the performance characterist1ca of the several models of Revere "Sun-Aid" Modular Solar Energy Collcotorc available.

SURFACES: 'Revere offers three surface treatments for the copper Tube-In-Strip absorber plate. The table below 11 sts these surface options with their respective absorptance and emittance values.

\begin{tabular}{l|c|c}
\multicolumn{1}{c|}{ Surface Option } & $\begin{array}{l}\text { Solar Spectrum } \\
\text { Absorptance }\end{array}$ & $\begin{array}{l}\text { Infrared } \\
\text { Emitiance }\end{array}$ \\
\hline Nextel Black Velvet' Paint & 0.96 & 0.95 \\
$\begin{array}{l}\text { Revere "E" Cuprous Oxlde } \\
\text { Selective }\end{array}$ & $0.88-0.91$. & $0.12-0.30$ \\
Black Chrome Selective & 0.95 & 0.07
\end{tabular}

plot number one demonstrates the difference for the varlous surface options. The three curves shown are for a collector with a single special low iron glazing.

GLAZINGS: Revere offers five glass cover options. All glass is 1/8" thick, tempered. The double glazed units are the realed type with an enclosed desiccant. The table below lists the various glass options with their respective solar spectrum transmissivity.

\begin{tabular}{l|c}
\multicolumn{1}{c|}{ Cover Option } & $\begin{array}{c}\text { Percent solar spectrum } \\
\text { Transmissivity }\end{array}$ \\
\hline Single Special Low Iron & 89.58 \\
Single Water White Crystal & 91.58 \\
Double Low Iron & 73.18 \\
Double Special Low Iron & 80.18 \\
Double Water Whito Cryotal & 83.78
\end{tabular}




\section{Revere Copper and Brass Incorporated}

Plot \#2

\section{REVERE 'BUN-AID' MODUIAR SOLAR ENERGY COLIECTORS \\ BLACK CHROME BELECTIVE SUREACE}

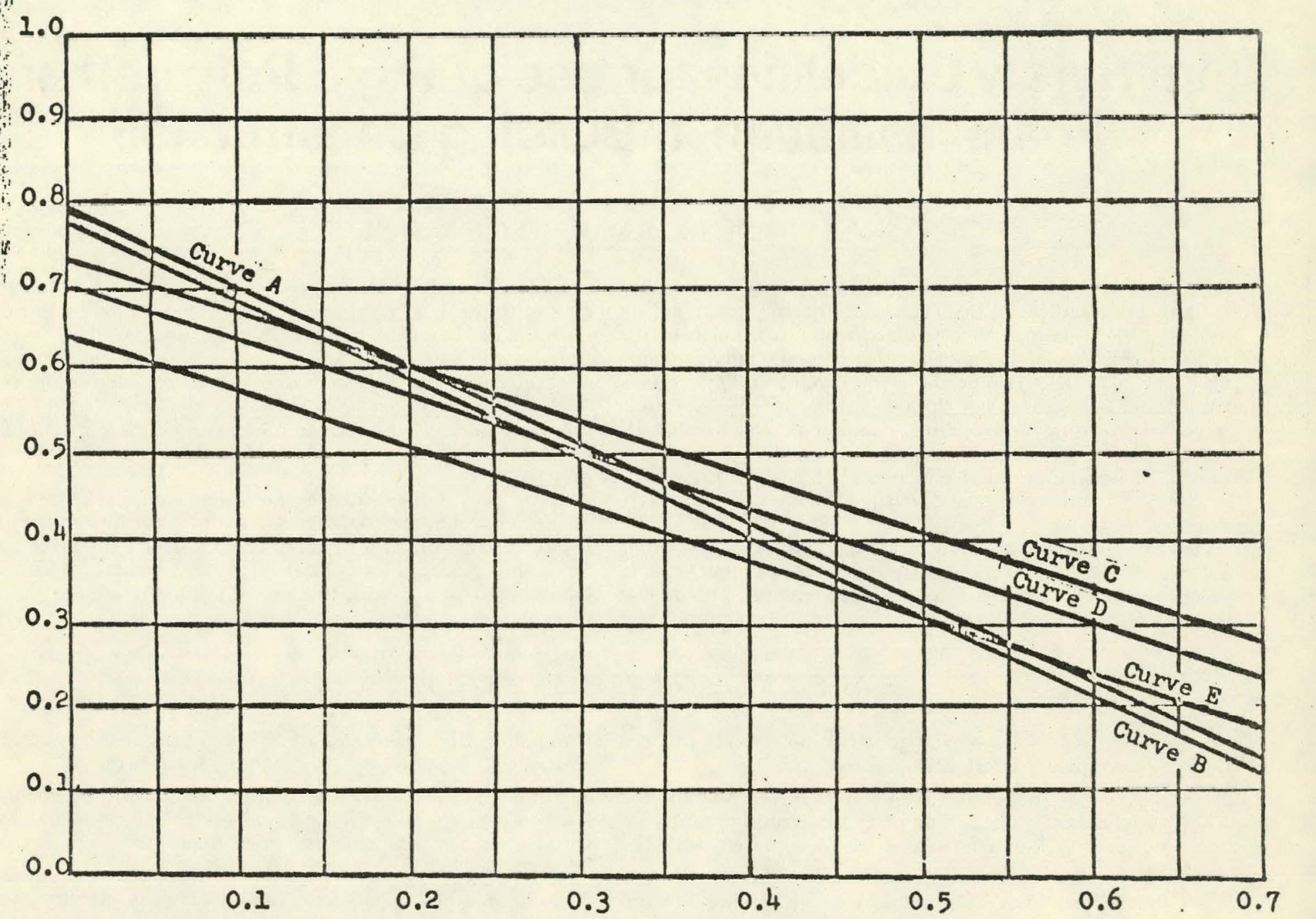

FLUID PARAMETER, $x_{1}=\left[\frac{\left(T_{1 n}+T_{\text {out }}\right)}{2}-T_{\text {amb }}\right] / I,{ }^{\circ} \mathrm{F} \cdot f t^{2} \cdot h r / B T U$

Curve A Tempered Single Water Wh1te Glass, NBS Tested, $\mathbb{N}_{1}=.792-.933 \mathrm{X}_{1}$

Curve B Tempered Single Speclal Low Iron Glass, $\mathbb{N}_{1}=.775-.933 X_{1}$

Curve C Tempered Double Water White Glass, $N_{1}=.735-.669 x_{1}$

Curve D Tempered Double Spectal Low Iron Glass, $N_{1}=.703-.669 X_{1}$.

Curve $\mathrm{E}$ Tempered Double Low Iron Glass, $\mathrm{N}_{1}=.642-.669 \mathrm{x}_{1}$ 


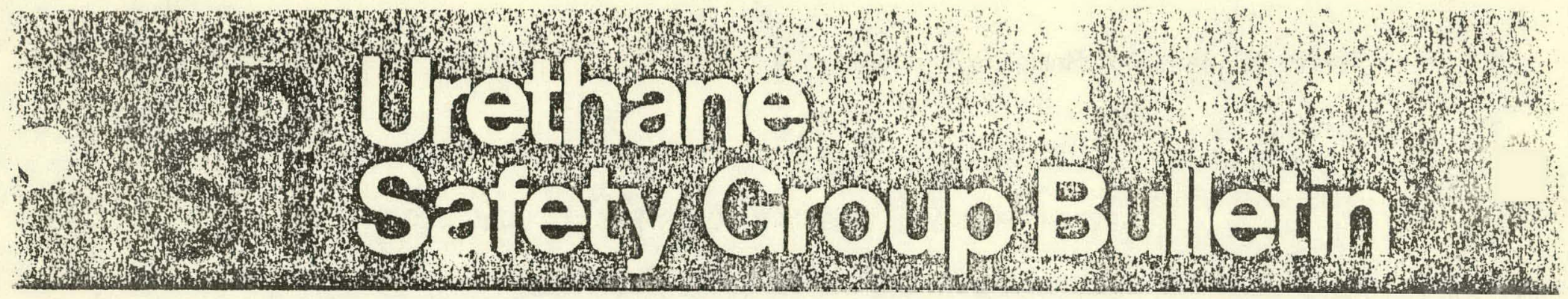

(U-100R)

\title{
Fire Safety Guidelines for Use of Rigid Polyurethane Foam Insulation in Building Construction
}

\author{
PULYURE I HANE FOAM AND COMBUSTIBILITY
}

Rigid foam, polyurethane or isocyanurate based, is an exceptionally effective insulation material for the construction industry. Inch for inch, it provides greater resistance to the iransfer of heat and cold than any other commercially available insulating material. This leads to maximum energy savings while permitting such design options as thinner walls and roofs or smaller heating and cooling equipment. Because of its closed-cell structure, it has low moisture permeability and thus retains its insulating value effectively. It is light in weight, versatile, self-supporting and easily inscalled by properly trained and equipped craftsmen.

Depending on formulation, combustibility characteristics of polyurethane foams vary widely, as do those of other organic materials. At the present stage of development, all organic foams, whether they contain fire retardants or not, should be considered combustible and handled accordingly. Experience demonstrates that certain precautions must be taken to minimize the fire hazard in handling, storage and use.

How polyurethane is used in a building system ultimately determines its fire sufety. Exposed polyurethane foam must be protected from accidental ignition by completely covering it with a flame barrier as soon as possible after installation, preferably the same day. Sprinkler protection may also be desirable.

Rigid polyurethane can be formulated on site from liquid chemicals which are foamed in place by pouring or spraying. It also is available as a rigid boardstock which can be cut and fitted into place, or as preformed panels, some of which are laminated with materials that shield against ignition.

\section{SAFETY DURING CONSTRUCTION}

Fire is a serious concern during construction. Good practices suggest the following safety precautions:

1. Foamed-on-site polyurethane should be mixed and applied only by applicators trained in its proper use and familiar with its limitations. It should be foamed in accordance with the supplier's recommendation.

2. Prohibit open flames, cutting and welding torches, electric heaters, high intensity lamps, and smoking materials such as cigarettes, pipes and cigars, from foam storage and installation areas. If hot work must be done near exposed polyurethane, shield the foam from heat and sparks by a thermal barrier such as asbestos cement board. A fire watch is desirable. Do not weld or cut metal which is in contact with polyurethane.

3. Provide fire extinguishing equipment at both storage and installation sites. Water in a fine spray usually is an effective method of extinguishing polyurethane foam fires.

4. Store foam boardstock in limited quantities, in a location free from any ignition hazard and preferably protected by a sprinkler system. Do not stack more than 8 feet high. Provide adequate aisle space for access between stacks.

5. Store and open liquid foam materials out of direct sunlight at temperatures not excededing $85^{\circ} \mathrm{F}$ in a well-ventilated location. Do not mix liquid waste components together for disposal because spontancous combustion could occur. 1)econtaminate empty drums by filling with water cut of doors and allowing to stand 48 hours uncapped.

6. Waste fnam shrulat he disposed of daily in a designated location with due regard for its combustible characteristics.

\section{SAFETY IN DESIGN}

Each formulation of polyurethane foam has its own maximum service temperature which should be observed. Consult your supplier for this information.

The most important consideration is to make sure that a suitable flame barrier covers all surfaces of polyurethane foam insulation. Additionally, certain applications may require sprinkler protection. L ocal building code and fire code officials, insurers, and manufacturers' specifications and installation instruction should be checked in each specific instance.

Further guidance maty be obtained from provisions of three national model building codes ${ }^{1}$ which specify requirements for foams used in specific applications. 


\section{SAFETY IN DESIGN (cont'd.)}

Following are some fire safety design guides for the architect and contractors based on these model codes and recommendations of the Urethane Safety Group.

\section{For Interior Use}

1. Polyurethane foam used in all interior wall and/or ceiling construction or concealed spaces should not be left exposed but should be covered with at least $1 / 2$ inch of cement plaster or fire-rated gypsum wallboard or an equivalent 15 -minute thermal barrier.

2. Polyurethane foam installed above a suspended ceiling, such as in a refrigerated building requires protection by a thermal barrier above the foam, i.e., between the top side of the foam and the underside of the floor above. The result should be a thermal barrier on both sides of the foam.

3. For panels comprising metal facings on foam cores, the model building codes specify minimum thicknesses of steel or aluminum facings, and maximum flame spread and smoke developed ratings for the cores, and require automatic sprinkler protection. The codes provide for exceptions to these requirements based on acceptable diversified testing.

4. The high insulating value of polyurethane foam on a ceiling or roof can result in rapid heat buildup under the high points of the structure if a fire should occur beneath this surface. Automatic heat vents at high points of ceiling are recommended to provide for release of heat and smoke before dangerous buildup occurs.

5. Fire stops should be provided for large warehouse ceiling areas, between floors in multi-story buildings, in concealed spaces, and at penetrations into pipe chases and ventilation shafts.

- 6. Polyurethane foam should not be used in contact with chim- neys, heater vents, steam pipes (unless specifically designed for this application) or other areas which could be subject to service temperatures exceeding ratings recommended by supplier.

7. Polyurcthane foam may be used to fill cavities within masonry walls or under grade level concrete floors.

NOTE: In all construction, new total system concepts to provide effective fire safety for buildings-incorporating automatic early detection, alarm and suppression devices-are recommended.

\section{For Exterior Use}

1. Polyurethane foam may be used as a roof covering over concrete, poured gypsum, tongue-and-groove wood or other properly prepared roof decks if the foam insulation is part of a class A, B or C roof assemtly as tested by ASTM E-108. 2. Foam insulation may be used as part of a roof assembly over bare metal decks provided, (a) a fire-rated underlay of perlite, gypsum board or other approved thermal barrier material is applied between the foam and the deck to provide protection from fire inside the building, or (b) the roof assembly meets the requirements for protection from fire inside the building of the code authorities who have jurisdiction over the specific application.

3. For other types of roofing applications, refer to local or model codes.

4. When used as an exterior insulating material on such structures as tanks or chemical processing equipment, polyurethane foam requires protection from the weather and ultra-violet rays of the sun and incidental ignition, depending on the circumstances. Consult your materials manufacturer, insurer and fire officials.

\section{COMBUSTIBILITY TESTS, RATINGS AND HAZARDS}

Numerous federal regulations and regional, state and local building codes make reference to combustibility tests and standards such as ASTM E-84 (or UL 723) the Steiner Tunnel test. While tests, and numerical flame spread ratings derived from these tests, are the most common means available today to compare the various combustibility characteristics of plastics and other materials, and to communicate these characteristics to knowledgable consumers, they are valid only as measurements of the performance of materials under specific, controlled test conditions and are not intended to reflect hazards under actual fire conditions. More than one test, and possibly a full-scale room or corner test, may be necessary to qualify a material for intended or proposed use.

It also should be understood that rigid polyurethane foam, as other organic materials used in construction, are combustible and, if ignited, will release smoke, toxic gases (the most significant of which usually is carbon monoxide), and flammable gases which could result in flashover, all of which may cause hazards to life as well as damage to property. While the recommendations contained herein are generally recognized by federal agencies, the model building codes and other regulatory bodies as providing requisite levels of safety to life and property, the National Bureau of Standards has stated 'hat no standard test methodology is currently available to svaluate the hazards presented by the toxic off-gases of combustion.

The Urethane Safety Group, in conjunction with The Society of the Plastics Industry, Inc., is engaged in research to evaluate present tests and to develop new tests that will more accurately predict performance of tested materials in actual fire conditions.

'Information on the model building codes may be obtained from:

Uniform Building Code

International Confesence of Building Officials

5360 South Workman Mill Road

Whittier, California 90601

Basic Building Code

Building Officials Conference of America

1313 East 60th Street

Chicago, Illinois 60637

Standard Building Code

Southern Building Code Congress

36178 th Avenue, South

Birmingham, Alabama 35222

Further information on the proper application of rigid urethane fodm may be obtained from your materials supplier and:

The Society of the Plastics Industry, Inc.

355 Lexington Avenue, New York, New York 10017

Nrtional Fire Protection Association

470 Atlantic Avenue, Boston, Massachusetts 02210.

Factory Mutual Research Corporation

P.O. Box 688, Norwood, Massachusetts 02062.

National Bureau of Standards

U.S. Department of Commerce, Washington, D.C. 20234. 


\section{URETHANE}

NOTE: For information on Urethane Foam, including specifications and manufacturers, contact the Urethane Foam Contractors Association (UFCA) at:

1406 Third National Building - Dayton, Ohio 45402 - phone (513) 223.0419

\section{DIATHON}

PHYSICAL PROPERTIES:

1. VOLUME SOLIDS: $60 \%$

This high solids by volume of $60 \%$ results in 9.5 mils per gallon per $100 \mathrm{sq}$. ft. of surface. Coverage figures are based on smooth surfaces. Textured surfaces such as urethane foam may require $15 \%$ to $30 \%$ more material. 20 to 28 dry mils, properly applied, is usually sufficient to protect a polyurethane foam roof.

2. FIONGATION: $120 \%$ at $0^{\circ} \mathrm{F}$ (ASTM D.2370) $200 \%$ at $40^{\circ} \mathrm{F}$. $280 \%$ at $75^{\circ} \mathrm{F}$. $360 \%$ at $10 n^{\circ} \mathrm{F}$

3. FLEXIBILITY:

Films aged under simulated 10 year weathering conditions retain their ability to be flexed $180^{\circ}$ without cracking. (ASTM D.822)

1. SHORE A HARDNESS: 45 (ASTM D.2240)

5. DRY PFEL ADHESION: 20 pli (ASTM D.413) Adhesive strength is greater than cohesive strength.

6. HIGH TEMPERATURE STABILITY: Resists roof temperature heat and does not age harden or slump at roof temperatures up to $200^{\circ} \mathrm{F}$. (ASTM D-794)

\section{PERFORMANCE}

WEATHER PROTECTIVE COATING

Provide type of elastomeric coating that when applied to exterior sprayed urethane foarn surfaces, will meet following requirements:
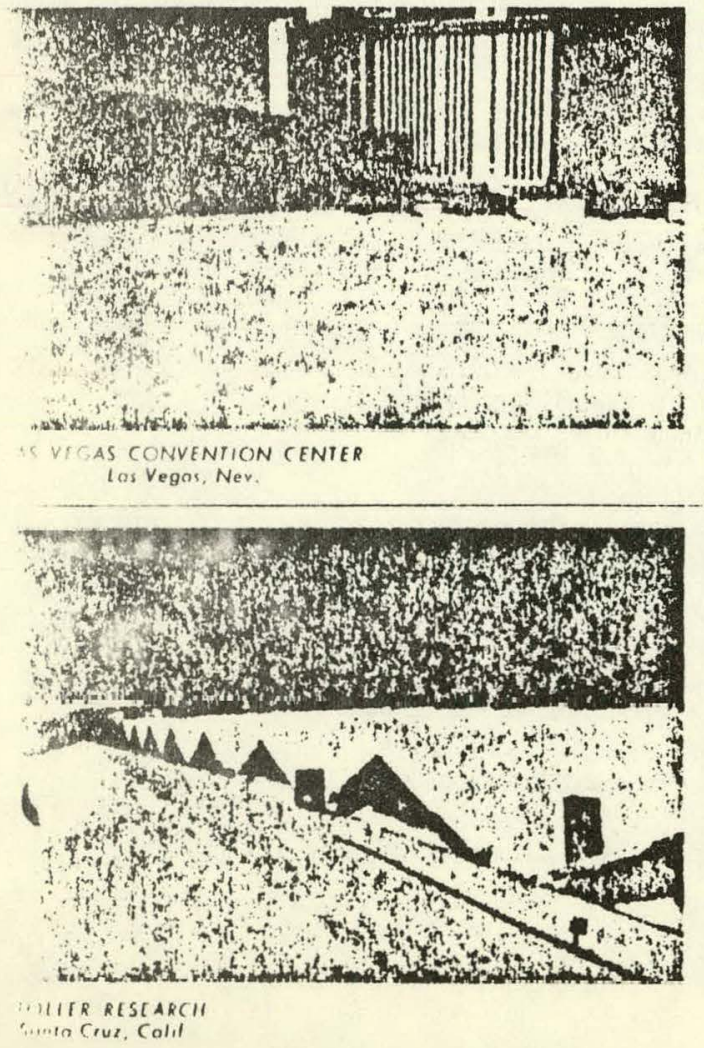

a. Will have excellent resistance to all forms of weather between $180^{\circ} \mathrm{F}$. to $-70^{\circ} \mathrm{F}$. and will retain elastomeric properties for a time period equivalent to 15 to 20 years as tested in accord with ASTM D.822.

b. Will prevent absorption of exterior moisture into pores of urethane foam as tested using wind driven rain machine in accord with Fed. Spec. Standard TTC.555. B.

c. Will have excellent resistance to "coastal" salt spray weather as tested in accord with ASTM D-1654.

d. Will allow moisture vapor from building interior to pass through coating and will provide a perm rating of 3.0 at 20 dry mils, as tested in accord with ASTM E-398 or ASTM D.1653.

e. Will not oxidize as tested in accord with ASTM D.822.

f. Will show no appreciable change in color after 1000 hours of testing in accord with ASTM D.822.

g. Will resist roof temperature heat up to $200^{\circ} \mathrm{F}$. in accord with ASTM D.794 and will not age harden or sluinp up to $200^{\circ} \mathrm{F}$.

h. Will retain ability to be flexed $180^{\circ} \mathrm{F}$. without cracking after simulated 10 year weathering test as tested in accord with ASTM D.822.

i. Will have a dry peel adhesion strength of minimum 20 pli as tested in accord with ASTM D.413.
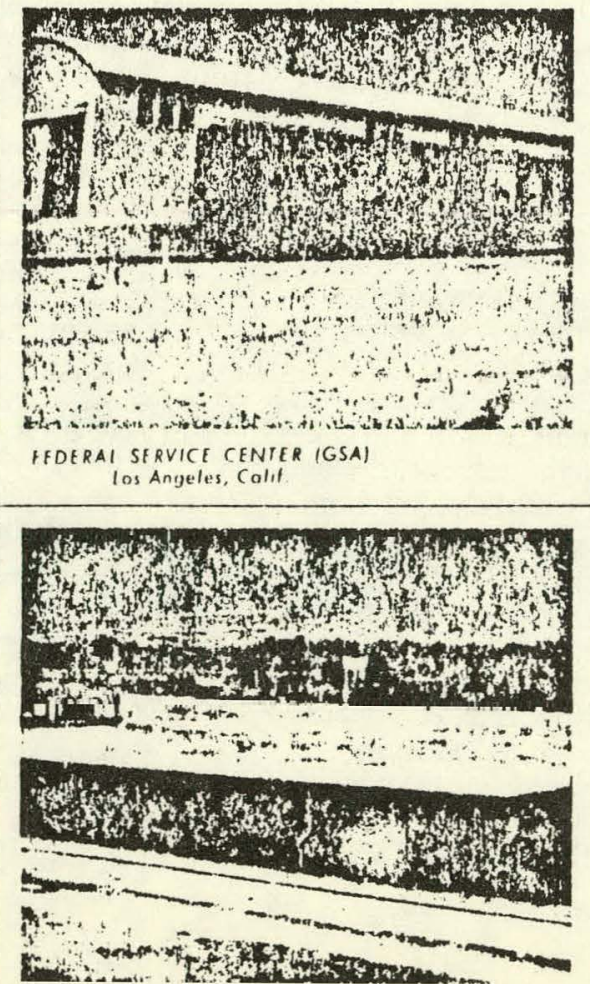

SHOPPINC CENTER Defiance, Ohio

$10-22$ j. Will have minimum elongation of $12.0 \%$ at $\mathrm{O}^{\circ} \mathrm{F}$., $20\left(\mathrm{)} \%\right.$ at $40^{\circ} \mathrm{F}$. $280 \%$ at $75^{\circ} \mathrm{F}$., $360 \%$ at $100^{\circ} \mathrm{F}$. as tested in accord with ASTM D.2370.

k. Will have minimum Shore $A$ hardness of 45 as tested in accord with ASTM D.2240.

I. Will conform to all local and federal air pollution requirements.

m. Will conform to requirements for a Class $\mathrm{A}$ rating on non-combustible substıates as tested in accord with U.L. 790

n. Will have a flame spread of $20 \mathrm{nr}$ less on non combustible substrates as tested in accord with ASTM E-84

o. Will prevent degradation of urethane foam substrates due to ultra violet penetration when foam is coated with a continuous film without pinholes or voids as tested in accord with ASTM D.822.

\section{CERTIFICATION}

A notarized certification, quart sample. and coated urethane foam sample shali be submitted from the manufacturer ten days prior to bid opening.

Certification shall state that properties specified above are met.

\section{PRODUCT COMPOSITION \\ SPECIFICATION}

\section{WEATHER PROTECTIVE COATING}

Shall be a high solids elastomer rubber produced by an emulsion polymerization technique combining elastomer acrylic, reinforcing! laminar pigmenls, dinl non.migratıng fire retardants. There shall be no solvents or migratory plasticizers used. Use of non-elastomeric acrylic resins will not be permitted.

Additional Minimum Physical Properties of Coating Shall Be:

a. Solids by volume

b. Ury nuls per gallon per $100 \mathrm{sq}$. $\mathrm{ft}$. of surface.

$60 \%$

Weight per gallon.

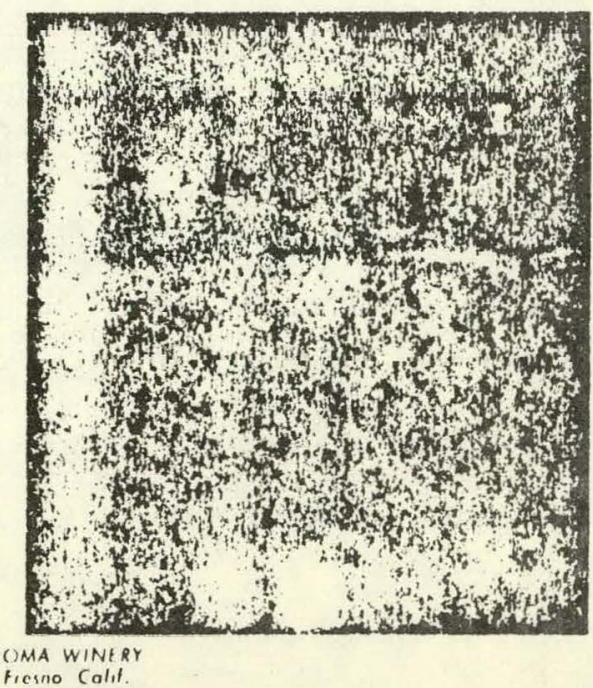




\section{Pqua Coll Installation Diagram}

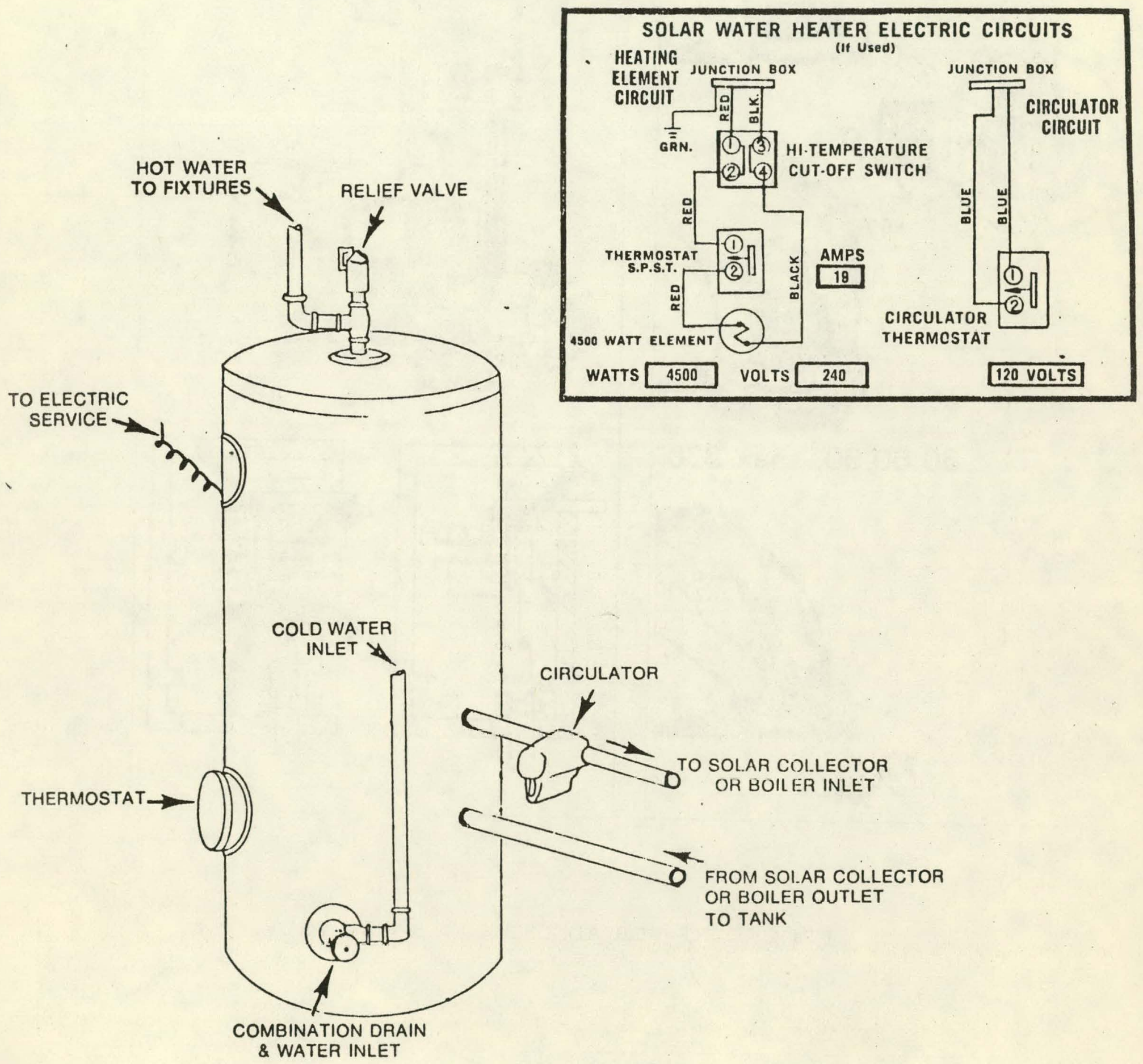



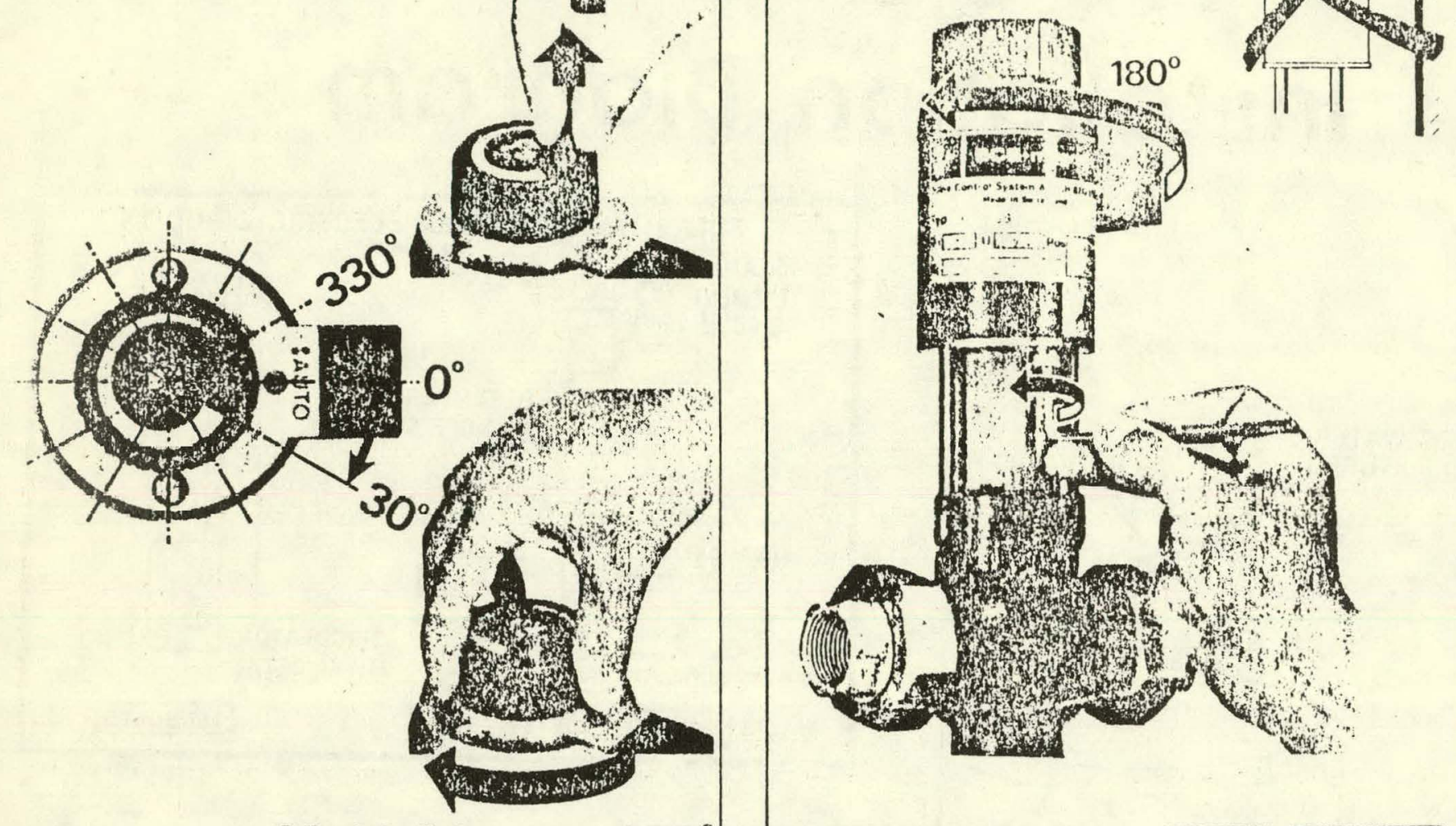

$30,60,90 \ldots \max .330^{\circ}$
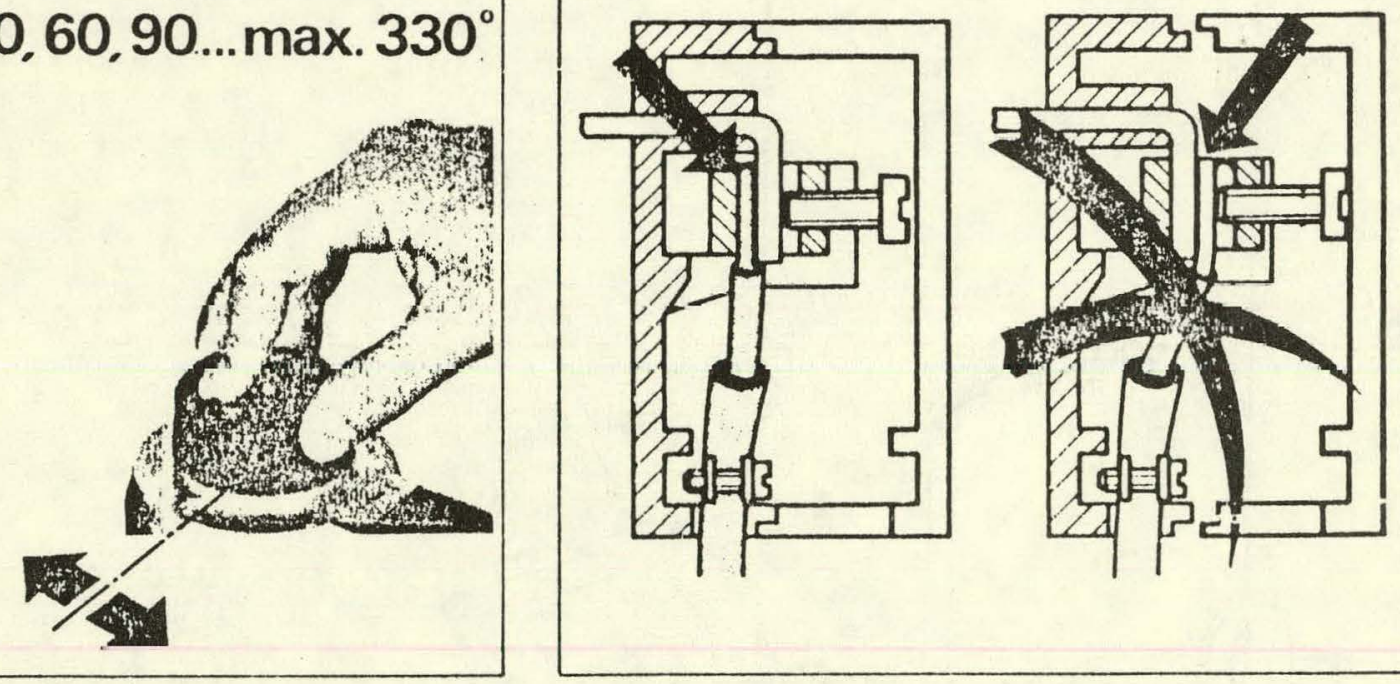

PROCEDURE FOR ADJUSTING A 3-WAY VALVE 
PROCEDURE FOR MOUNTING AND ADJUSTING PNEUMATIC ACTUATORS
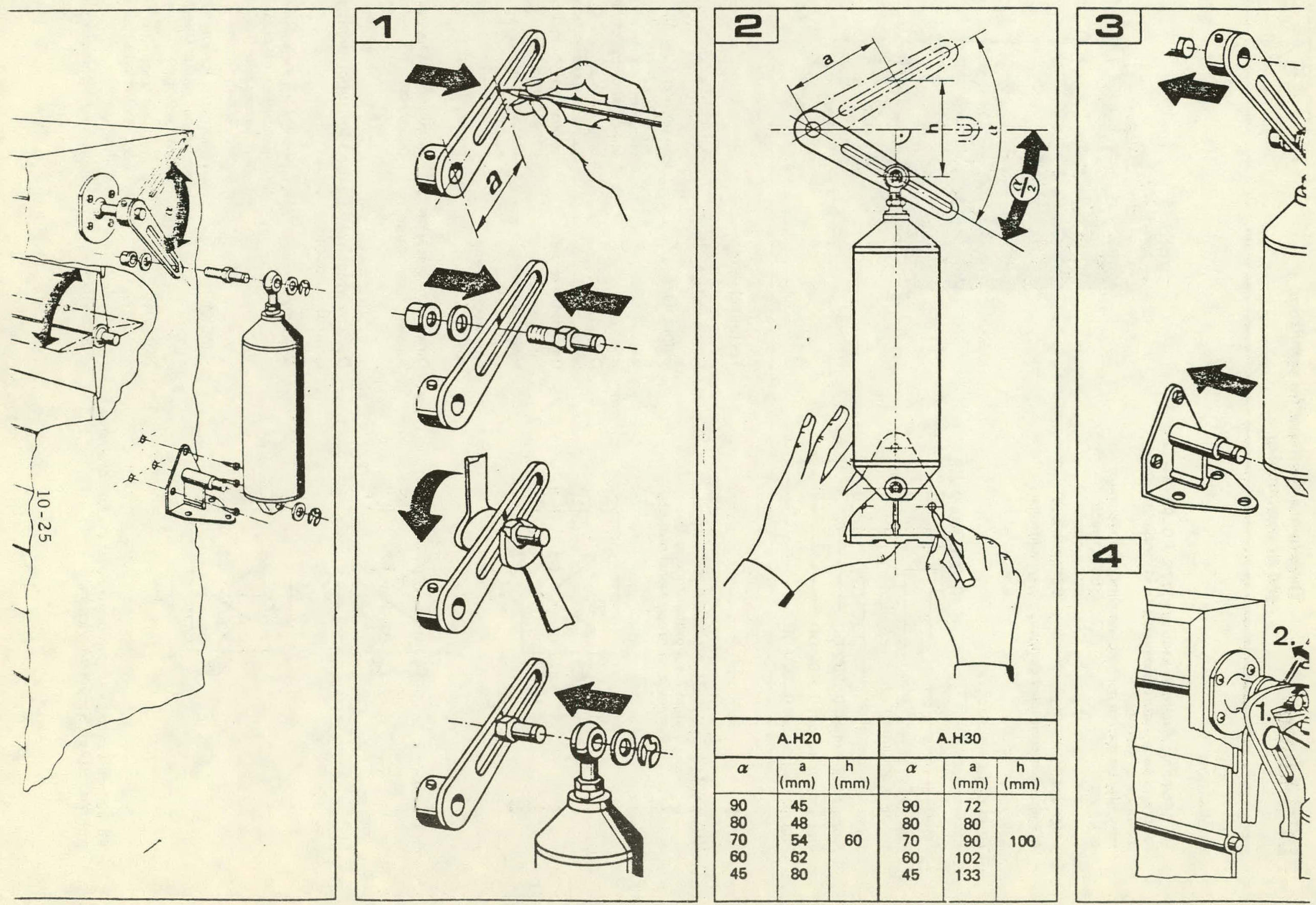

\section{4}

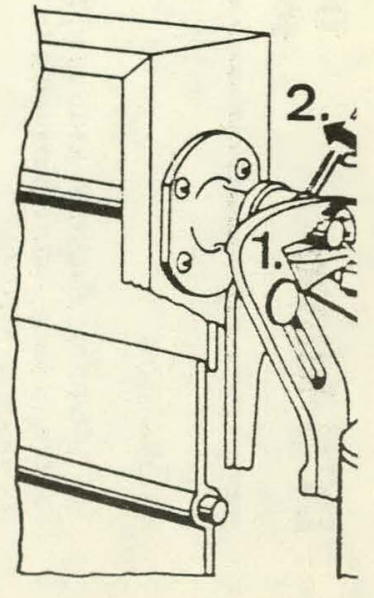




\section{Application}

The SCS-klimo electronic controller, type RDL $9 / 9 / 9$ is used to control temperature and humidity in ventilation and air conditioning systems.

They may be used in conjunction with the following:

- Valves

- SCS-magnetic

- SCS-motoric

- Motors

- SCS-push pull

- Electronic controlled devices . step controllers

\section{Type code}

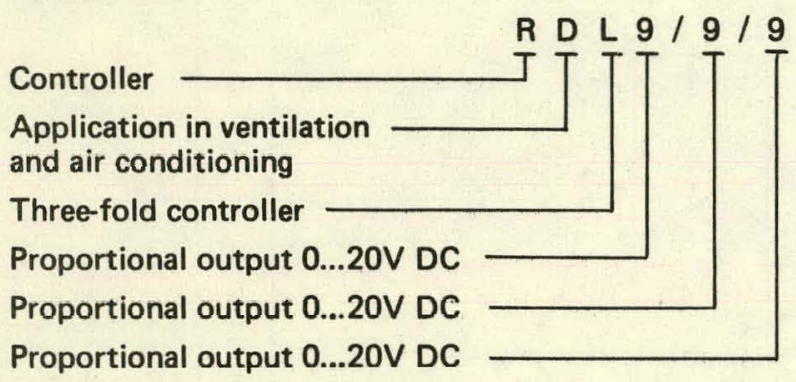

\section{Type}

RDL9/9/9 System 1: 1 proportional output System 2: 1 proportional output System 3: 1 proportional output
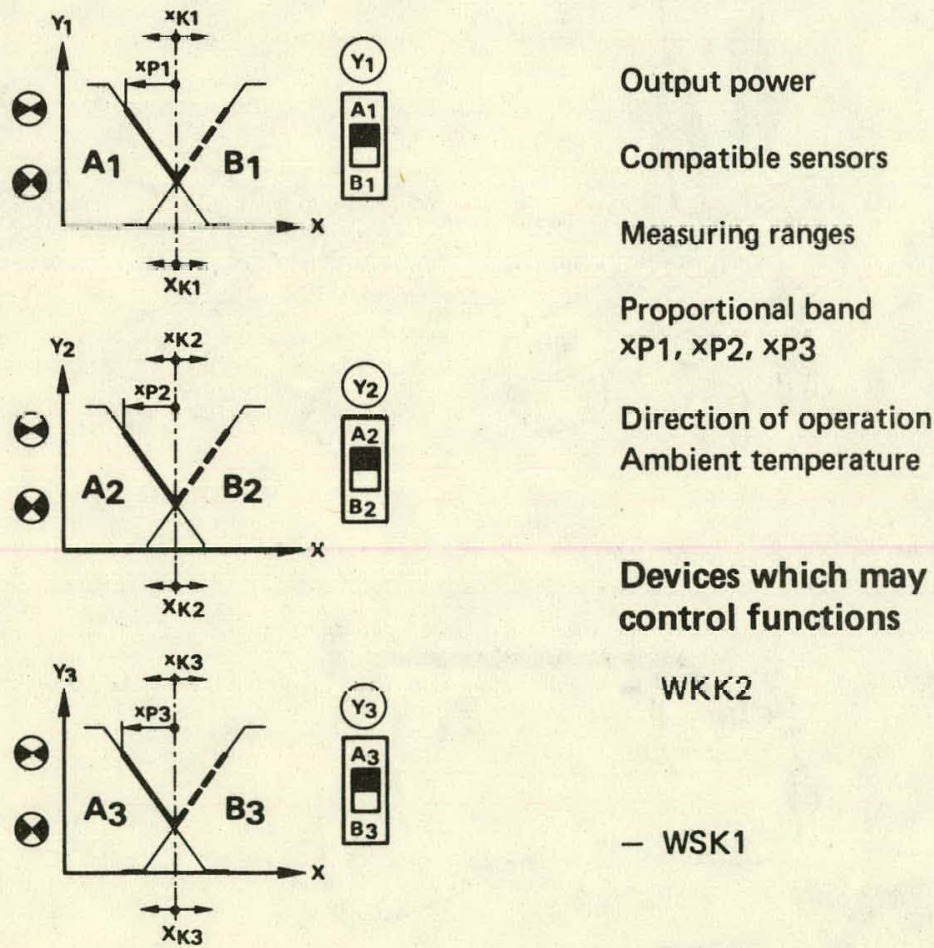

All controller outputs can be adjusted over the full scale range with the relevant $X_{K}$ potentiometers.

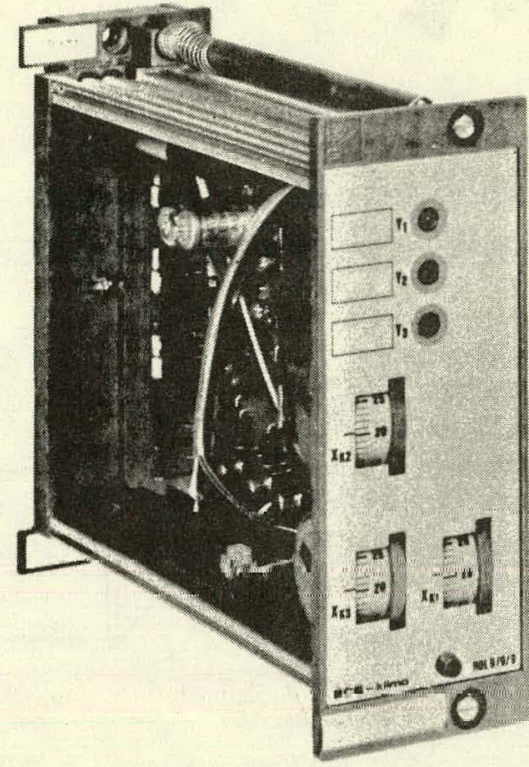

Technical data

Supply voltage

Output signal

Output power

Compatible sensors

Measuring ranges

Proportional band XP1, XP2, XP3

Ambient temperature

$24 \mathrm{~V}+10 \% 50 \ldots 60 \mathrm{~Hz}$ proportional (ref: 9)

$Y_{1}, Y_{2}, Y_{3}$ : each $0 . .20 \mathrm{VDC}$ phase cut indicated by indicator lamp $\max .40 \mathrm{~W}$ at $20 \mathrm{~V} \mathrm{DC}$ for all outputs added together temperature, humidity and pressure sensors

temperature: $\quad 0 . . .3 \mathrm{O} \cup \mathrm{C}$ humidity: $\quad 30 \ldots 80 \% \mathrm{rH}$

adjustable $\quad 0.5 \ldots 9 \mathrm{~K}$

all outputs are reversible 1... $18, \% \mathrm{rH}$ 0 ... 50 oC

\section{Devices which may be used for supplementary control functions}

$\begin{array}{cl}\text { WKK2 } & \begin{array}{l}\text { reset transmitter for summer or win- } \\ \text { ter compensation for gradual increase } \\ \text { in room temperature (for max. of }\end{array} \\ & 5 \text { control loops). } \\ \text { - WSK1 } & \begin{array}{l}\text { reset transmitter for summer and } \\ \text { winter compensation (for max. of } \\ \end{array} \\ & 100 \text { control loops) } \\ \text { - WSU1 } & \begin{array}{l}\text { universal reset transmitter for more } \\ \text { complex reset/interference pro- } \\ \text { grammes } \\ \text { indicators for monitoring }\end{array}\end{array}$




\section{Adjustments}

The setpoints are adjustable externally.

The controller is supplied with temperature scale strips. These have a scale for relative humidity on the back and should therefore be reversed for humidity measurements. For pressure measurements the scale strip should be replaced by the one supplied with pressure sensors.

The front plate can be removed by applying light pressure to the red knob ab its lower edge. All the pqtentiometers are now accessible (proportional band XP1, XP2, XP3, calibration $\left.x_{K} 1, x_{K}, x_{K}\right)$. Indicator lamps indicate the operating status, thus facilitating adjustment considerably.

There are operating switches on the printed circuit board, for changing the direction of operation of each individual controller output.

On the printed circuit board there are also 3 pinboard with plug-in resistors. Sensor-setpoint combinations are achieved by altering the position of a maximum of three resistors (in order, for example, to render the built-in potentiometer inoperative when a remote setpoint potentiometer is used). The position of the resistors should be altered to suit the type of sensor, setpoint potentiometer or setpoint potentiometer combination in question.

\section{Pinboard}

On the printed circuit board in the controller there are three pinboards with plug-in resistors. The position of the resistors is altered to suit the type of sensor used.
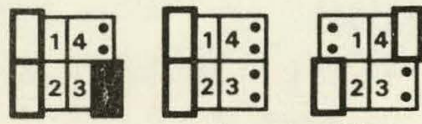

F... $\underbrace{\text { PU... DR... }}_{\text {pinboards }}$ position on delivery

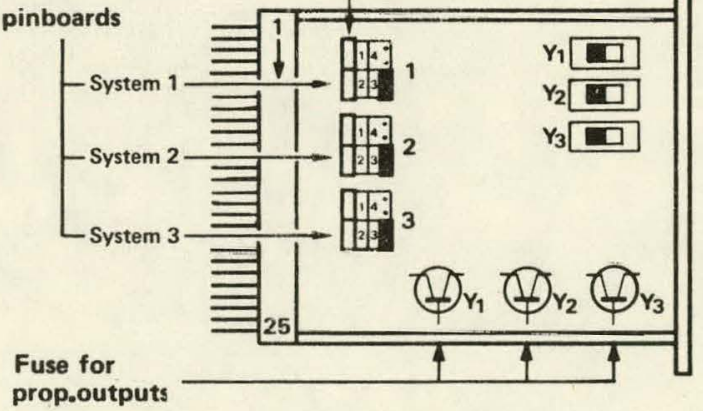

\section{Principle of operation}

The RDL9/9/9 controller is a self-contained module comprising - power supply

- 2 measuring loops

- 3 control amplifiers (assigned to the measuring loops)

Integrated circuits amplify the signal from a temperature, humidity or pressure sensor to give a proportional output signal $0 \ldots 20 \mathrm{~V} \mathrm{DC}$ (reference 9). This allows direct connection of all controlled devices (e.g. valves), damper motors, step controllers and power controllers. There is a separate indicator lamp for each individual controller output.

\section{Construction}

The RDL9/9/9 controller is compact in design and takes up the minimum of space in the front of the control panel while making optimum use of its depth.

It comprises a baseplate and a plug-in control unit. Keyed sockets and pins on the baseplate and the control unit prevent the wrong equipment from being plugged in by mistake.

Two retaining rods ensure a secure connection between the control unit and the baseplate.

\section{Mounting}

First the baseplate is mounted and connected up. The control unit is then plugged into the baseplate and secured with the retaining rods.

\section{Dimensions [mm]}

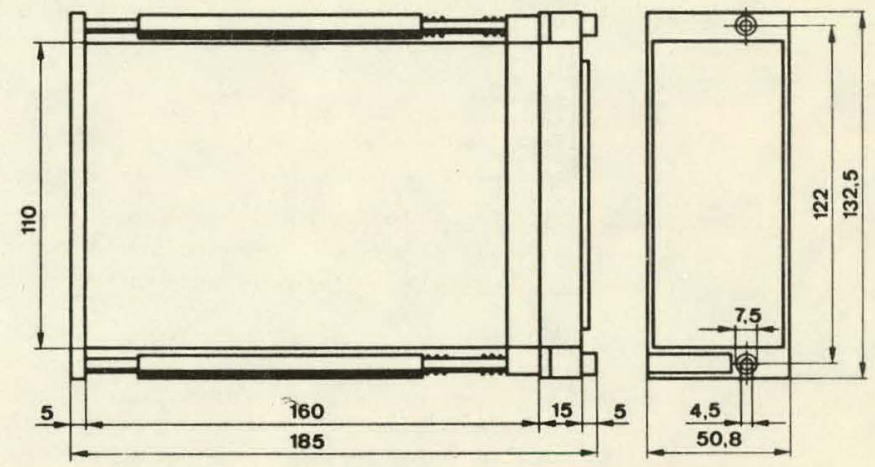

\section{Baseplate}

(drilling diagram)

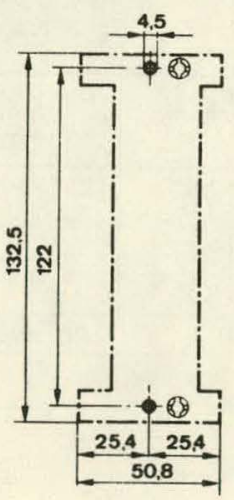

Front panel installation dimensions

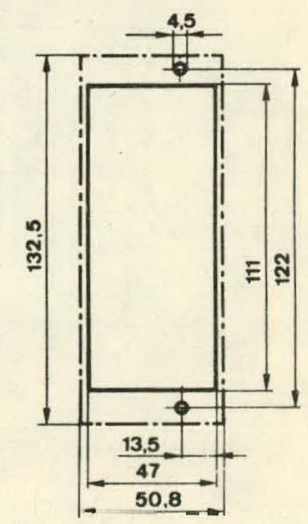




\section{Connection diagrams}

Terminal lay-out RDL9/9/9

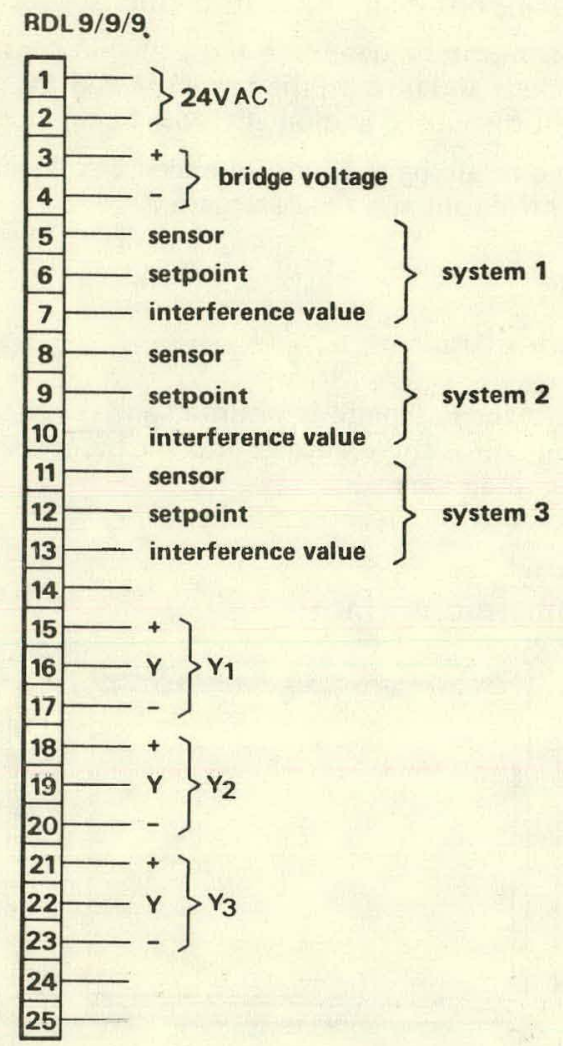

\section{Controller inputs}

Temperature sensors
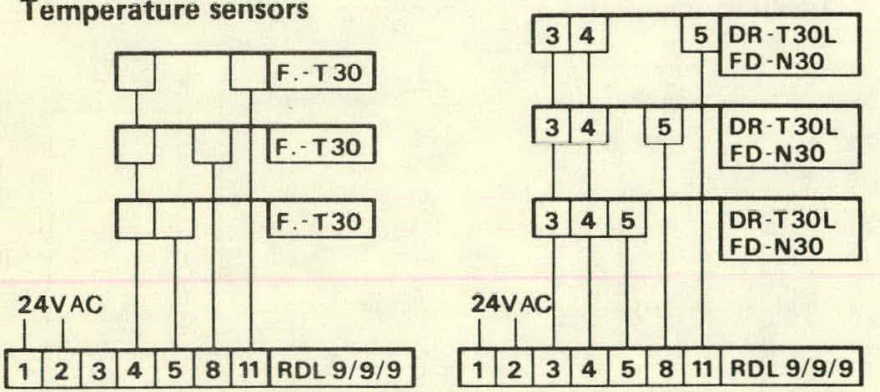

Setpoint potentiometer for temperature

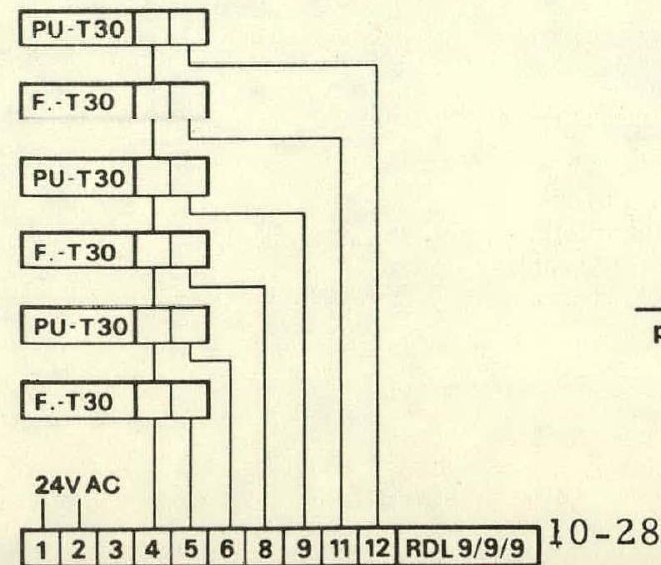

Humidity sensors

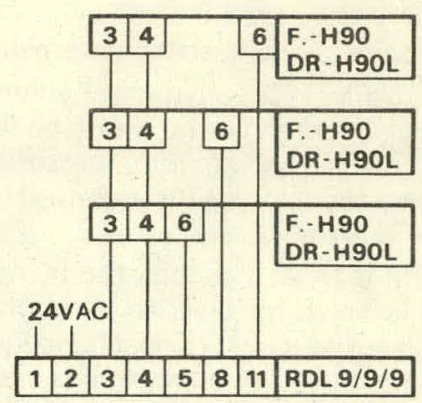

Temperature and humidity sensors

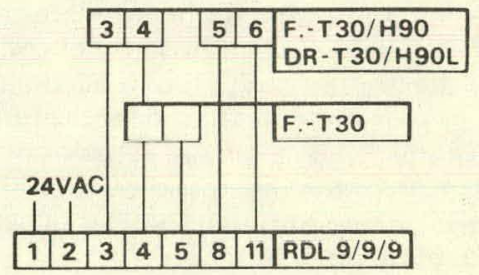

Setpoint potentiometer for humidity

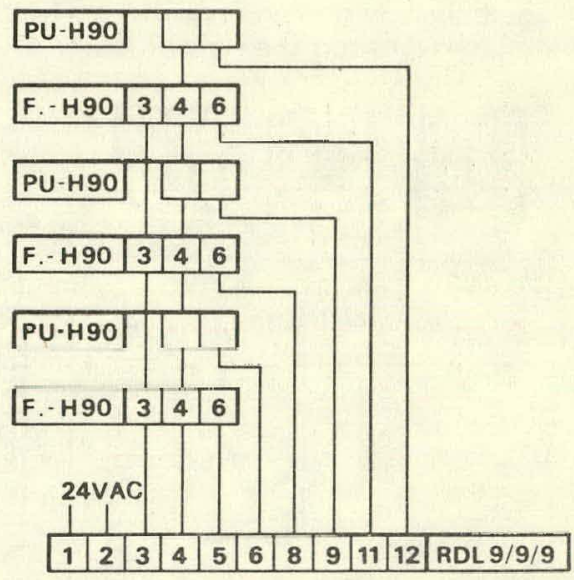

\section{Controller outputs}

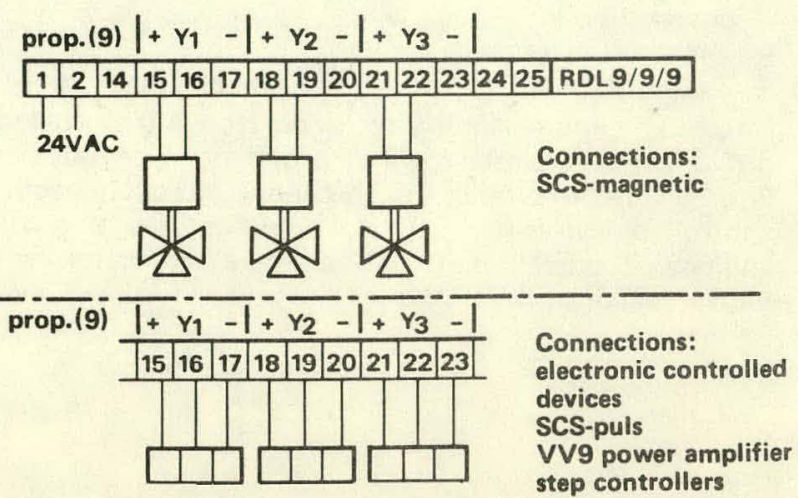




\section{Application}

The WSA3 averaging controller is used in multizone systems. It forms the average of a maximum of 10 control signals and passes the resultant control signal, which is equivalent to the average energy requirement of the plant, to either output $Y_{1}$ (heating) or output $\mathrm{Y}_{2}$ (cooling).

This signal is transmitted to the RDE2 controller which compares the energy available in the outside or return air with the actual demand and selects the optimum sequence of operation.

\section{Technical data}

Supply voltage

Control voltage

Power consumption

Output voltage

$$
\begin{aligned}
& Y_{1} \\
& Y_{2}
\end{aligned}
$$

Output power

$\mathrm{Y}_{1}$

Starting point

\section{$\mathrm{Y}_{2}$}

$\mathrm{X}_{\mathrm{K} 1}$

$\mathrm{X}_{\mathrm{K} 2}$

Steepness $\left[\frac{\Delta U_{E M}}{\Delta U_{A}}\right] S_{1}$

Direction of operation

Number of inputs $U_{E}$

Ambient temperature $t_{a m b}$

$$
\begin{aligned}
& 24 \mathrm{~V}+15 \%, 50 \ldots 60 \mathrm{~Hz} \\
& 0 \ldots 20 \mathrm{~V} \text { DC phase cut } \\
& 6 \mathrm{VA}
\end{aligned}
$$

0 ... 20V DC phase cut $0 \ldots 20 \mathrm{~V}$ DC phase cut $40 \mathrm{VA}$ 40 VA - max. 40 VA adjustable $0 \ldots 20 \mathrm{~V} D C$ adjustable 0 ... 20V DC

adjustable $0.15 \ldots 1$ adjustable $0.15 \ldots 1$ reversible $\max .10$ $0 \ldots 50^{\circ} \mathrm{C}$

\section{Indication}

Two indicator lamps $Y_{1}$ and $Y_{2}$ on the front of the controller indicate the output voltages $\mathrm{U}_{\mathrm{A} 1}$ and $\mathrm{U}_{\mathrm{A} 2}$ in proportion to their brightness.

Basic diagram

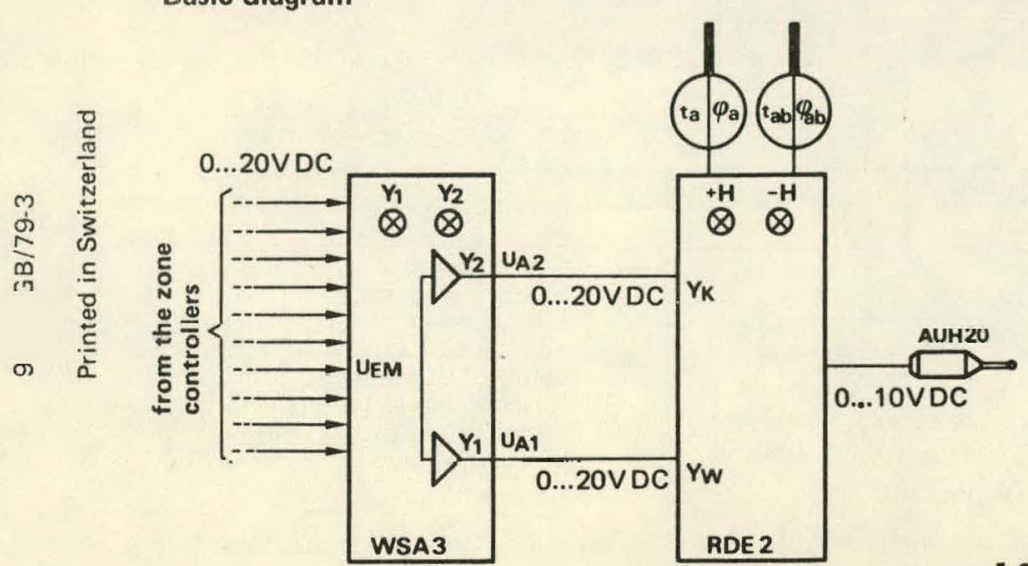

\section{Function diagram}
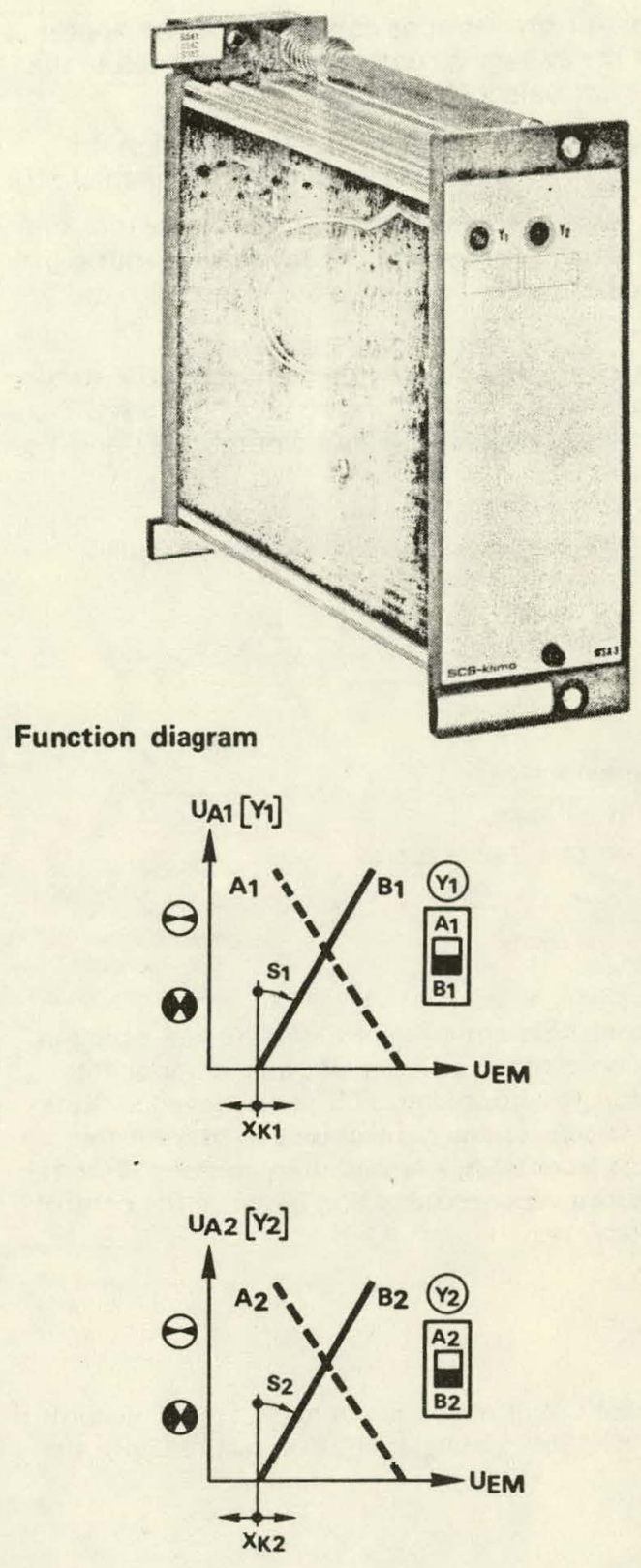

Adjustment example

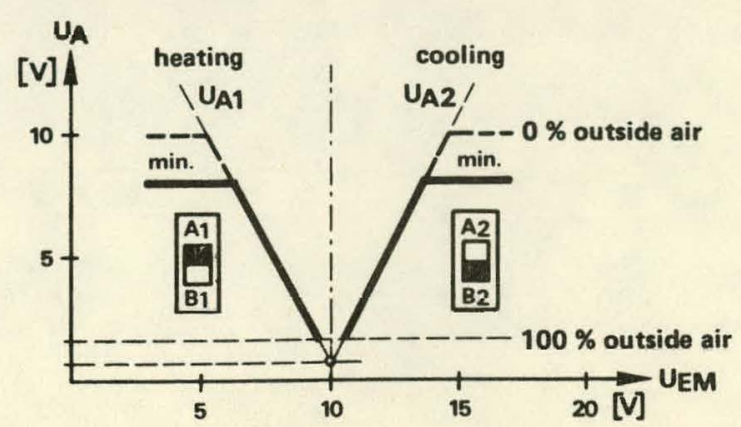




\section{Principle of operation}

The output signals from the zone controllers, which appear as input signals at the averaging controller are converted in the input filter into equivalent DC voltages.

The inputs used are switched on at the 10-position multiswitch. (Inputs that are not being used must be switched off.)

The main zones should be given priority. To ensure that suitable allowance is made for very large zones, the output signal from the associated zone controller is connected to more than one input.

In the preliminary amplifier stage the steepness $S$, the starting point $X_{K}$ and the direction of operation $A$ or $B$ are set.

The processed signals are available at the outputs $Y_{1}$ and $Y_{2}$ for energy recovery.

The average control signal is equivalent to the formular:

$$
U_{E M}=\frac{U_{E 1}+U_{E 2}+\ldots U_{E n}}{n}
$$

in which:

- UEM - average voltage

- $U_{E}=$ input voltage

$-n=$ number of inputs used

\section{Construction}

The averaging controller comprises a baseplate and a plug-in control unit which contains all the electronic components and the adjustable potentiometers. There are keyed sockets and pins on the baseplate and control unit to prevent the wrong equipment from being plugged in by mistake. Two reraining rods ensure a secure connection between the control unit and the baseplate.

\section{Mounting}

First the baseplate is mounted and connected up. The control unit is then plugged into the baseplate and secured with the retaining rods.

\section{Dimensions [mm]}

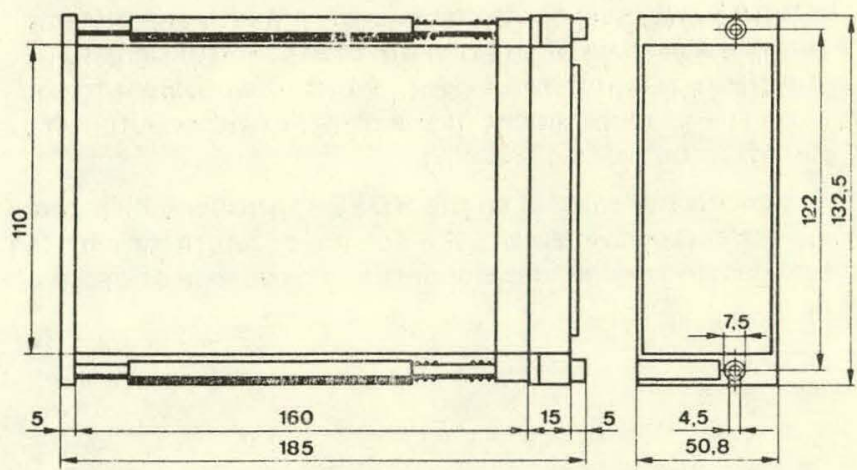

Baseplate

(drilling diagram)

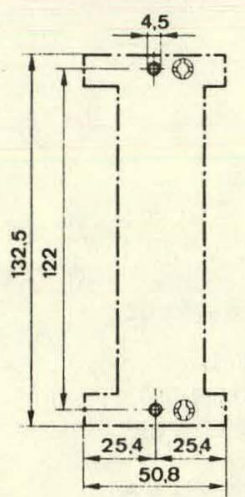

Front panel installation dimensions

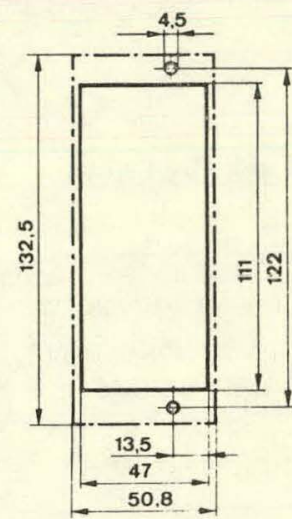

\section{Connection diagram}
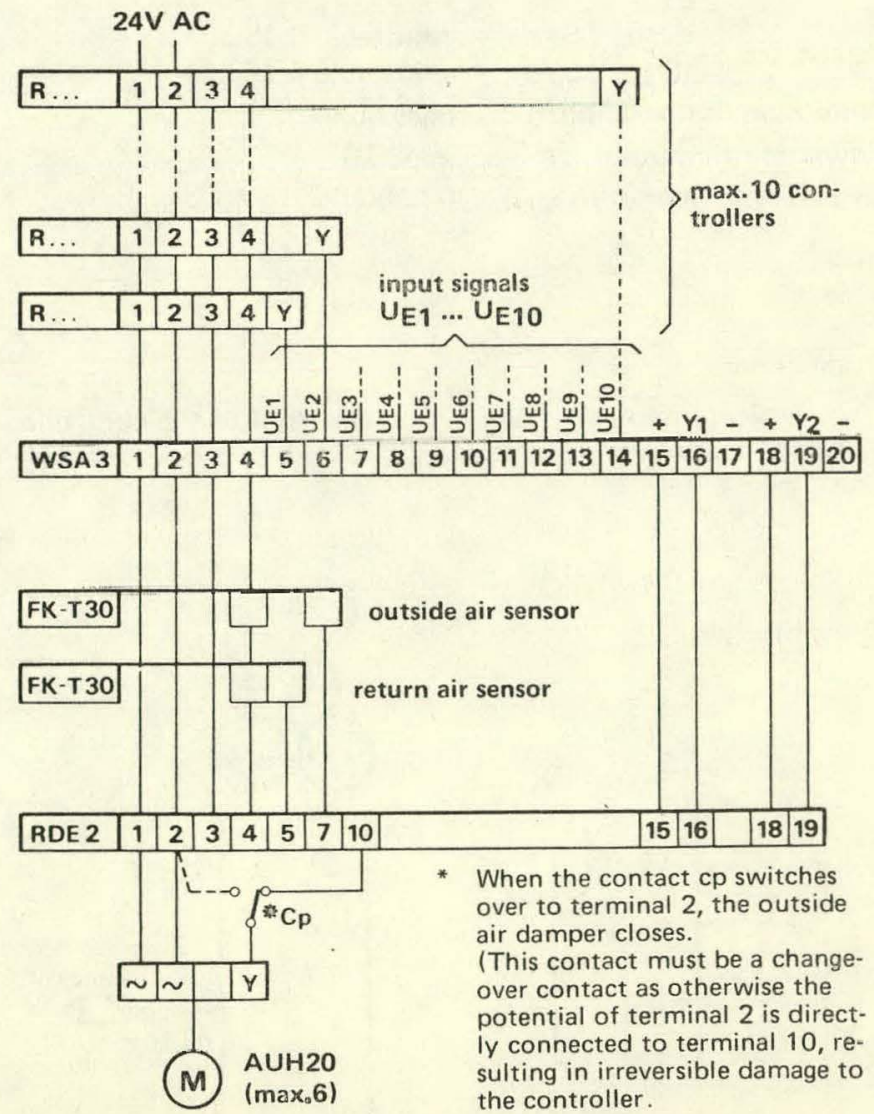

Important

The zone controllers and the averaging controller must be supplied from the same transformer. 


\section{M P O RT A NT ... SUMOSTRANO INSTALLATION INFORMATION}

\section{SUNDSTRAND ROTA-ROLL' FUEL UNITS}

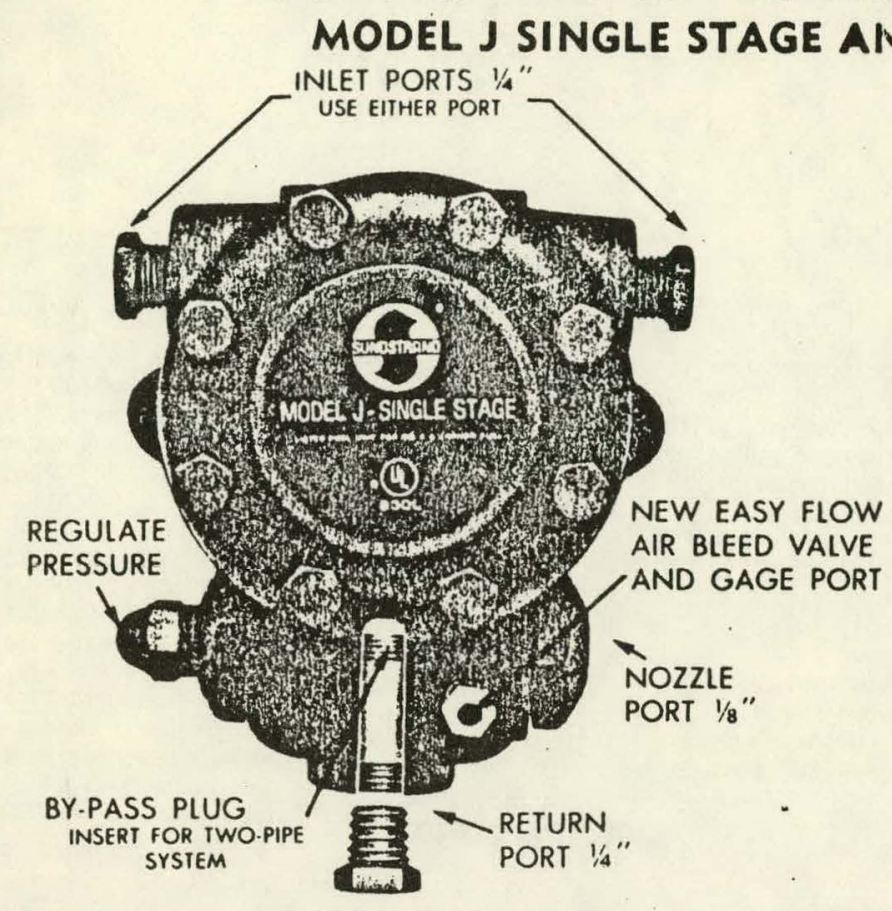

ONE-PIPE SYSTEM (Inlet line only)

Check to see that by-pass plug has not been installed for two-pipe system. Units are set for a one-pipe system. Line length under 50 feet use 3/8" O.D. copper tubing. Line length 50-100 feet use $1 / 2$ " O.D. copper tubing.

TWO-PIPE SYSTEM (Inlet and Return line) Remove internal by-pass plug from cloth bag. Insert as shown in illustration and tighten securely. Refer to line size information on reverse side.

\section{ALL SYSTEMS - General Information}

1. Oil lines should consist of not smaller than $3 / 8^{\prime \prime}$ O.D. copper tubing. See line size and installation data.

2. Oil lines must be absolutely air tight. Check all connections and joints.

3. Return line and inlet pressures must not exceed 10 P.S.I. Higher pressures may cause the seal to leak.

4. The model "J" pump should be used where inlet vacuum does not exceed 10" Hg. The model "H" pump should be used where inlet vacuum does not exceed 20 " of $\mathrm{Hg}$ at 1725 ı pm. The maximun inlet vacuum at $3450 \mathrm{rpm}$ is 15 "

\section{AIR BLEED PROCEDURE WITH NEW EASY FLOW AIR BLEED VALVE}

\section{One-Pipe System}

Start burner: Loosen Easy Flow Air Bleed Valve CCW just $1 / 4$ turn for fast purging.

For clean bleed in restricted spaces, an easily attached hose can be used to direct bleed oil into a container. A 3/16" I.D. hose can be slipped directly over end of valve.

Optional Procedure: On gravity feed systems, before starting burner, loosen unused intake port plug until there is a flow of oil from the port.

\section{Two-Pipe Systems}

Air bleeding is automatic. Opening Easy Flow Air Bleed Valve will allow oil to be pulled up faster. .

\section{MOUNTING POSITION}

Model "J" may be mounted in any position.

Note: Direction-of rotation and nozzle port location determined from shaft end with valve at bottom.

Current Model " $\mathrm{H}$ " with arrows on cover may be mounted with the valve horizontal at either top or bottom.

Valve may be mounted vertical providing the adjusting screw is at the top on $\mathrm{CW}$ rotation-left nozzle and $\mathrm{CCW}$ rotation-right nozzle models, or adjusting screw at bottom on $\mathrm{CW}$ rotation-right nozzle and $\mathrm{CCW}$ rotation-left nozzle models.

Earlier " $H$ " models having a designation ending in $-1-2$ and -3 were only intended for mounting with valve underneath.

\section{ADDITIONAL INSTALLATION INFORMATION \\ Model $\mathrm{E}$ and $\mathrm{F}$ for HEAVY OIL.......... Form No. 1011 \\ Piping of fuel lines for oil fired WATER HEATER \\ Form No. 1335 \\ Sundstrand Boost Pump - \\ SIMPLIFIED CIRCUIT \\ Sundstrand QUICK PURGE VALVE \\ Hum Eliminator for RETURN LINE. \\ Form No. 450012 \\ Form No. 450015 \\ Form No. 450021} of $\mathrm{Hg}$.
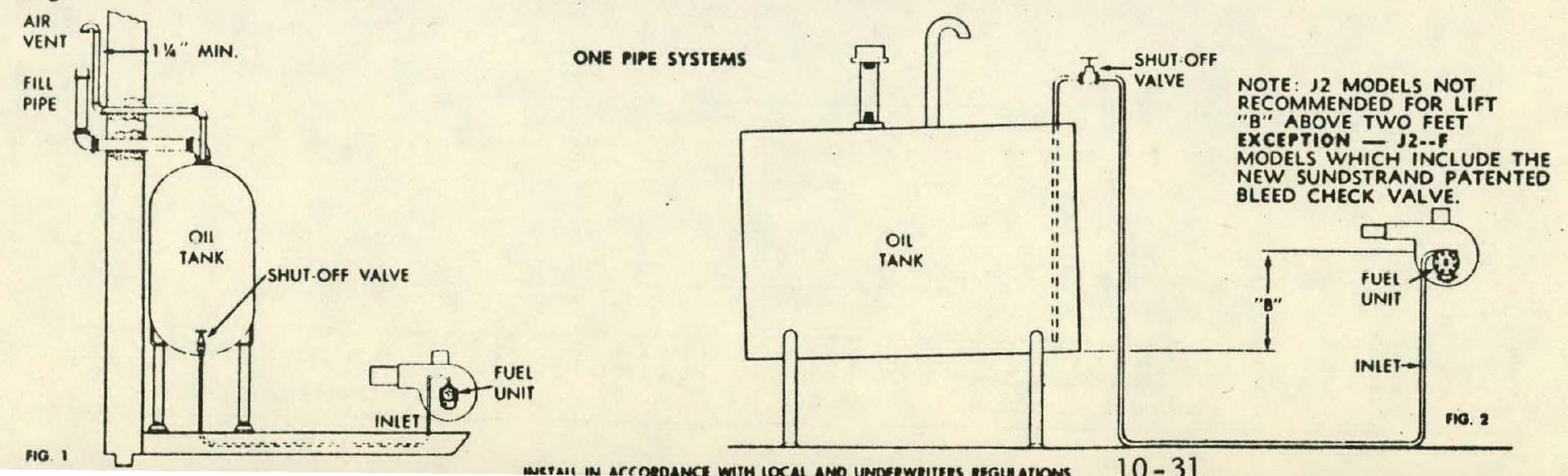
INSIDE OR OUTSIDE TANK FUEL UNIT ABOVE BOTTOM OF TANK

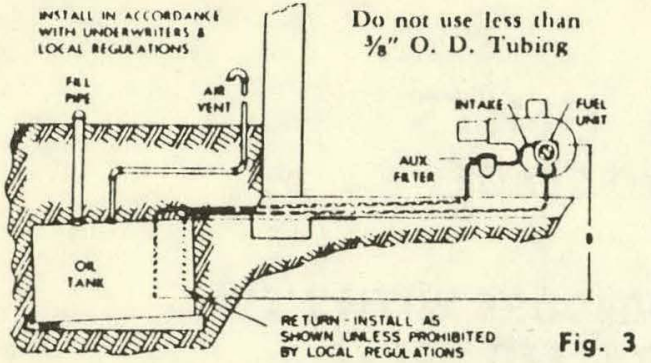

TWO PIPE INSTALLATION

MAXIMUM ALIOWABLE

LENGTH OF TOTAL

INTAKE OR RETURN

LINE IN FEET.

ICALCULATED FOR FUEL

OIL, VISCOSITY 57 SSUI

LIFT CONDITION (FIG. 3)

INSIOE OR OUTSIDE TANK FUEL UNIT BELOW

BOTYOM OF TANK

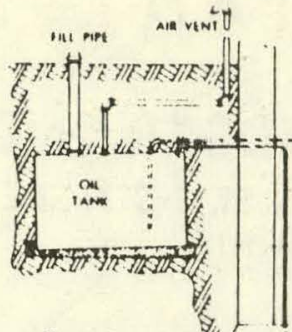

Fig. 4
1) not use less than $3 / 8$ " O. 1). Tubing

$1 / 2$ "O. I) Tubing (1725 RPM)

$1 / 2 " O$ 1). Tubing ( 3450 RPM)

\begin{tabular}{|c|c|c|c|c|c|c|c|c|c|c|c|c|c|c|c|c|c|c|c|c|c|c|c|c|c|c|c|c|c|c|c|c|c|c|c|c|c|c|}
\hline \multirow{2}{*}{$\begin{array}{l}\text { Lift " } B \text { " } \\
\text { Fig. } 3\end{array}$} & \multicolumn{10}{|c|}{ \%"O. D Tubing (1725 RPM) } & \multicolumn{12}{|c|}{$1 / 2 "$ O. I) Tubing (1725 RPM) } & \multicolumn{16}{|c|}{$1 / 2 "$ O 1). Tubing (3450 RPM) } \\
\hline & $\mathrm{J}_{2}$ & $\mathrm{~J}_{3}$ & $\mathrm{~J}_{4}$ & $\mathrm{~J}_{3}$ & $J_{6}$ & $\mathrm{H}_{2}$ & $\mathrm{H}_{3}$ & $\mathrm{H}_{4}$ & $\mathrm{H}_{3}$ & $\mathrm{H}_{6}$ & $\mathrm{~J}_{2}$ & $\mathbf{J}_{3}$ & $\mathrm{~J}_{4}$ & $\mathbf{J}_{3}$ & $\mathbf{J}_{6}$ & $\mathrm{H}_{2}$ & $\mathrm{H}_{3}$ & $\mathrm{H}_{4}$ & $\mathrm{H}_{6}$ & $\mathrm{H}_{6}$ & $\mathrm{H}_{7}$ & $H_{n}$ &. $\mathrm{JJ}_{2}$ & $\mathrm{JA}_{2}$ & $\mathrm{JB}_{2}$ & $J_{2}$ & $\mathrm{~J}_{3}$ & $\mathrm{~J}_{4}$ & $\mathrm{~J}_{3}$ & $\mathrm{~J}_{\mathrm{b}}$ & $\mathrm{HH}_{2}$ & $\mathrm{HA}_{2}$ & $\mathrm{HB}_{2}$ & $\mathrm{H}_{2}$ & $\mathrm{H}_{3}$ & $\mathrm{H}_{4}$ & $\mathrm{HI}_{3}$ & $H_{6}$ \\
\hline $0^{\prime}$ & $65^{\prime}$ & $65^{\prime}$ & $53^{\prime}$ & $42^{\prime}$ & 31 & $75^{\prime}$ & 77 & $75^{\prime}$ & 63 & $55^{\prime}$ & $100^{\prime}$ & 100 & $100^{\prime}$ & $100^{\prime}$ & 100 & $100^{\prime}$ & $100^{\circ}$ & $100^{\prime}$ & $100^{\prime}$ & $100^{\prime}$ & $100^{\prime}$ & $73^{\prime}$ & $100^{\prime}$ & $100^{\prime}$ & $100^{\prime}$ & $100^{\prime}$ & $100^{\prime}$ & $99^{\prime}$ & $77^{\prime}$ & $59^{\prime}$ & $100^{\circ}$ & $\left|100^{\prime}\right|$ & $100^{\prime}$ & $100^{\prime}$ & $100^{\prime}$ & $94^{\prime}$ & $81^{\prime}$ & $69^{\prime}$ \\
\hline$I^{\prime}$ & $60^{\prime}$ & $60^{\prime}$ & $49^{\prime}$ & $38^{\prime}$ & 29 & $72^{\prime}$ & $74^{\prime}$ & $72^{\prime}$ & 611 & $53^{\prime}$ & $100^{\prime}$ & $100^{\circ}$ & $100^{\prime}$ & $100^{\prime}$ & 100 & $100^{\prime}$ & $100^{\prime}$ & $100^{\circ}$ & $100^{\circ}$ & $1000^{\prime}$ & $100^{\prime}$ & $71^{\prime}$ & $100^{\prime}$ & $100^{\prime}$ & $100^{\prime}$ & $100^{\prime}$ & $100^{\prime}$ & $91^{\prime}$ & $72^{\prime}$ & $55^{\prime}$ & $93^{\prime}$ & $93^{\prime}$ & $93^{\prime}$ & $93^{\circ}$ & $93^{\prime}$ & $89^{\prime}$ & $77^{\prime}$ & $6 S^{\prime}$ \\
\hline $2 '$ & $55^{\prime}$ & $55^{\prime}$ & $45^{\prime}$ & $35^{\prime}$ & 27 & $69^{\prime}$ & $71^{\prime}$ & $70^{\prime}$ & 58 & $51^{\prime}$ & $100^{\prime}$ & 100 & $100^{\prime}$ & $100^{\prime}$ & 100 & $100^{\prime}$ & $100^{\prime}$ & $100^{\prime}$ & $100^{\prime}$ & $100^{\prime}$ & $100^{\prime}$ & $68^{\prime}$ & $100^{\prime}$ & $100^{\prime}$ & $100^{\prime}$ & $100^{\prime}$ & $100^{\prime}$ & $84^{\prime}$ & $66^{\prime}$ & $50^{\prime}$ & $88^{\prime}$ & $88^{\prime}$ & $88^{\prime}$ & $88^{\circ}$ & $88^{\circ}$ & $85^{\prime}$ & $73^{\prime}$ & $62^{\prime}$ \\
\hline $3^{\prime}$ & $50^{\prime}$ & $50^{\prime}$ & $41^{\prime}$ & $32^{\prime}$ & 24 & $67^{\prime}$ & $69^{\prime}$ & $67^{\prime}$ & 56 & $49^{\prime}$ & $100^{\prime}$ & 1007 & $100^{\prime}$ & $100^{\prime}$ & $96^{\prime}$ & $100^{\prime}$ & $100^{\prime}$ & $100^{\prime}$ & $100^{\prime}$ & $100^{\prime}$ & $100^{\prime}$ & $65^{\prime}$ & $93^{\prime}$ & $93^{\prime}$ & $93^{\prime}$ & $93^{\prime}$ & $93^{\prime}$ & $77^{\prime}$ & $60^{\prime}$ & $46^{\prime}$ & $83^{\prime}$ & $83^{\prime}$ & $83^{\prime}$ & $83^{\prime}$ & $83^{\prime}$ & $80^{\circ}$ & $6 y^{\prime}$ & $58^{\prime}$ \\
\hline $4^{\prime}$ & $45^{\circ}$ & $45^{\prime}$ & $37^{\prime}$ & $29^{\prime}$ & $22^{\prime}$ & $64^{\prime}$ & $66^{\prime}$ & $64^{\prime}$ & 53 & $47^{\prime}$ & $100^{\prime}$ & $100^{\circ}$ & $100^{\prime}$ & $100^{\prime}$ & 871 & $100^{\prime}$ & $100^{\prime}$ & $100^{\prime}$ & $100^{\prime}$ & $100^{\prime}$ & $100^{\prime}$ & $63^{\prime}$ & $84^{\prime}$ & $84^{\prime}$ & $84^{\prime}$ & $84^{\prime}$ & $84^{\prime}$ & $69^{\prime}$ & $54^{\prime}$ & $41^{\prime}$ & $78^{\prime}$ & $78^{\prime}$ & $78^{\circ}$ & $78^{\circ}$ & $78^{\prime}$ & $75^{\prime}$ & $64^{\circ}$ & $55^{\prime}$ \\
\hline $5^{\prime}$ & $40^{\prime}$ & $40^{\prime}$ & $33^{\prime}$ & $26^{\prime}$ & $20^{-}$ & $61^{\prime}$ & $63^{\prime}$ & $61^{\prime}$ & $51^{1}$ & $45^{\prime}$ & $100^{\prime}$ & $100^{\prime}$ & $100^{\prime}$ & $100^{\circ}$ & $78^{\prime}$ & $100^{\prime}$ & $10 n^{\prime}$ & $100^{\circ}$ & $100^{\circ}$ & $100^{\prime}$ & $96^{\prime}$ & $60^{\prime}$ & $75^{\prime}$ & $75^{\prime}$ & $75^{\prime}$ & $75^{\prime}$ & $75^{\circ}$ & $62^{\prime}$ & $48^{\prime}$ & $37^{\prime}$ & $73^{\prime}$ & $73^{\prime}$ & $73^{\prime}$ & $73^{\prime}$ & $73^{\circ}$ & $70^{\prime}$ & $60^{\prime}$ & $5 I^{\prime}$ \\
\hline $6^{\prime}$ & $36^{\prime}$ & $36^{\prime}$ & $29^{\prime}$ & $23^{\prime}$ & $17^{\prime}$ & $58^{\prime}$ & $60^{\circ}$ & $58^{\prime}$ & 49 & $43^{\prime}$ & $100^{\prime}$ & $100^{\prime}$ & $100^{\prime}$ & $90^{\prime}$ & $68^{\prime}$ & $1000^{\prime}$ & 100 & $100^{\prime}$ & $100^{\circ}$ & $100^{\circ}$ & $92^{\prime}$ & $57^{\prime}$ & $66^{\prime}$ & $66^{\prime}$ & $66^{\prime}$ & $66^{\prime}$ & $66^{\prime}$ & $54^{\prime}$ & $42^{\prime}$ & $32^{\prime}$ & $69^{\prime}$ & $69^{\prime}$ & $69^{\prime}$ & $69^{\prime}$ & $69^{\prime}$ & $66^{\prime}$ & $56^{\prime}$ & $48^{\prime}$ \\
\hline $7^{\prime}$ & $31^{\prime}$ & $31^{\prime}$ & $25^{\prime}$ & $20^{\prime}$ & 15 & $55^{\prime}$ & $57^{\prime}$ & $55^{\prime}$ & $46^{\prime}$ & $41^{\prime}$ & $100^{\circ}$ & $100^{\prime}$ & $99^{\prime}$ & $78^{\prime}$ & $59^{\prime}$ & $100^{\prime}$ & 100 & $100^{\prime}$ & $100^{\prime}$ & $100^{\prime}$ & $87^{\prime}$ & $54^{\prime}$ & $57^{\prime}$ & $57^{\prime}$ & $57^{\prime}$ & $57^{\prime}$ & $57^{\prime}$ & $47^{\prime}$ & $37^{\prime}$ & $28^{\circ}$ & $64^{\prime}$ & $64^{\prime}$ & $64^{\circ}$ & $64^{\prime}$ & $64^{\circ}$ & $61^{\prime}$ & $52^{\prime}$ & $44^{\prime}$ \\
\hline $8^{\prime}$ & $26^{\prime}$ & $26^{\prime}$ & $21^{\prime}$ & $17^{\prime}$ & 13 & $52^{\prime}$ & $54^{\prime}$ & $53^{\prime}$ & $44^{\prime}$ & $39^{\prime}$ & $100^{\circ}$ & $100^{\prime}$ & $83^{\prime}$ & $66^{\prime}$ & $50^{\prime}$ & $100^{\prime}$ & $100^{\prime}$ & $100^{\circ}$ & $100^{\prime}$ & $100^{\prime}$ & $8.3^{\prime}$ & $5 \mathrm{I}^{\prime}$ & $48^{\prime}$ & $48^{\prime}$ & $48^{\prime}$ & $48^{\prime}$ & $48^{\circ}$ & $40^{\circ}$ & $31^{\prime}$ & $24^{\prime}$ & $59^{\prime}$ & $59^{\prime}$ & $59^{\prime}$ & $59^{\prime}$ & $59^{\prime}$ & $56^{\prime}$ & $44^{\prime}$ & $41^{\prime}$ \\
\hline $9^{\prime}$ & $21^{\prime}$ & $21^{\prime}$ & $17^{\prime}$ & $14^{\prime}$ & - & $50^{\prime}$ & $51^{\prime}$ & $50^{\prime}$ & $42^{\prime}$ & $37^{\prime}$ & $83^{\prime}$ & $83^{\prime}$ & $68^{\prime}$ & $53^{\prime}$ & $40^{\prime}$ & $100^{\circ}$ & $100^{\prime}$ & $100^{\prime}$ & $100^{\prime}$ & $100^{\prime}$ & $79^{\prime}$ & $49^{\prime}$ & $39^{\prime}$ & $39^{\prime}$ & $39^{\prime}$ & $39^{\prime}$ & $39^{\circ}$ & $32^{\prime}$ & 25 & $19^{\prime}$ & $54^{\prime}$ & $54^{\prime}$ & $54^{\prime}$ & $54^{\circ}$ & $54^{\prime}$ & $52^{\prime}$ & $44^{\prime}$ & $36^{\circ}$ \\
\hline $10^{\prime}$ & $16^{\prime}$ & $16^{\prime}$ & $13^{\prime}$ & & $\cdots$ & $47^{\prime}$ & $48^{\circ}$ & $47^{\prime}$ & $39^{\prime}$ & $35^{\prime}$ & $64^{\prime}$ & $64^{\prime}$ & $52^{\prime}$ & $41^{\prime}$ & $31^{\prime}$ & $100^{\circ}$ & $100^{\prime}$ & $100^{\prime}$ & $100^{\prime}$ & $100^{\prime}$ & $74^{\prime}$ & $46^{\prime}$ & $30^{\prime}$ & $30^{\prime}$ & $30^{\prime}$ & $30^{\prime}$ & $30^{\prime}$ & $25^{\circ}$ & $19^{\prime}$ & $15^{\prime}$ & $49^{\prime}$ & $49^{\prime}$ & $49^{\prime}$ & $49^{\circ}$ & $4 y^{\prime}$ & $47^{\prime}$ & $40^{\prime}$ & $34^{\prime}$ \\
\hline $11^{\prime}$ & & & & & & $44^{\prime}$ & $45^{\prime}$ & $44^{\prime}$ & $37^{\prime}$ & $33^{\prime}$ & & & - & $\cdots$ & & $100^{\prime}$ & $100^{\prime}$ & $100^{\circ}$ & $100^{\prime}$ & $100^{\prime}$ & $70^{\prime}$ & $43^{\prime}$ & & & - & & & & - & - & $44^{\prime}$ & $44^{\prime}$ & $44^{\circ}$ & $44^{\prime}$ & $44^{\circ}$ & $42^{\prime}$ & $36^{\circ}$ & $31^{\prime}$ \\
\hline $12^{\prime}$ & & & & & & $41^{\prime}$ & $42^{\prime}$ & $41^{\prime}$ & $35^{\prime}$ & $31^{\prime}$ & & & & & & 100 & $100^{\prime}$ & $100^{\circ}$ & $100^{\circ}$ & $1000^{\prime}$ & $65^{\prime}$ & $40^{\prime}$ & & & & & & & -. & - & $39^{\prime}$ & $39^{\prime}$ & $39^{\circ}$ & $39^{\prime}$ & $39^{\prime}$ & $37^{\prime}$ & $32^{\prime}$ & $27^{\prime}$ \\
\hline $13^{\prime}$ & & & & & & $38^{\prime}$ & $39^{\prime}$ & $38^{\prime}$ & $32^{\prime}$ & $28^{\prime}$ & & & & & & $100^{\prime}$ & $100^{\prime}$ & $100^{\prime}$ & $100^{\prime}$ & $100^{\circ}$ & $61^{\prime}$ & $37^{\prime}$ & & & & & & 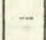 & - & $-\cdots$ & $34^{\prime}$ & $34^{\prime}$ & $34^{\prime}$ & $34^{\prime}$ & $34^{\prime}$ & $33^{\prime}$ & $28^{\prime}$ & $24^{\circ}$ \\
\hline $14^{\prime}$ & & & & & & $35^{\prime}$ & $37^{\prime}$ & $36^{\prime}$ & $30^{\prime}$ & $26^{\prime}$ & & & & & & 100 & $100^{\circ}$ & $100^{\circ}$ & $100^{\circ}$ & $99^{\prime}$ & $5 b^{\prime}$ & $34^{\prime}$ & & & & & & - & - & - & $29^{\prime}$ & $29^{\prime}$ & $29^{\prime}$ & $29^{\prime}$ & $2 y^{\prime}$ & $2 x^{\prime}$ & $24^{\prime}$ & $20^{\prime}$ \\
\hline $15^{\prime}$ & & & & & & $33^{\prime}$ & $34^{\prime}$ & $33^{\prime}$ & $28^{\prime}$ & $21^{\prime}$ & & & & & & $100^{\prime}$ & $100^{\prime}$ & $100^{\prime}$ & $100^{\prime}$ & $91^{\prime}$ & $52^{\prime}$ & $32^{\prime}$ & & & & & & & & $=$ & $24^{\prime}$ & 4. & $24^{\circ}$ & $24^{\prime}$ & $24^{\circ}$ & $233^{\prime}$ & 20 & $17^{\prime}$ \\
\hline
\end{tabular}

LIFT CONDITION (FIG. 3)

(1.18

\section{\$/" O D. Tubing (3450 RPM)}

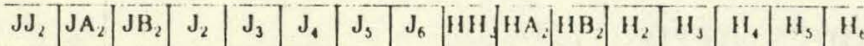
$100^{\prime} 100^{\prime} 100^{\prime} 100^{\prime} 100^{\prime} 100^{\prime} 100^{\prime} 100^{\prime} 100^{\prime} 100^{\prime} 100^{\prime} 100^{\prime} 100^{\prime} 100^{\prime} 100^{\prime} 100^{\prime}$

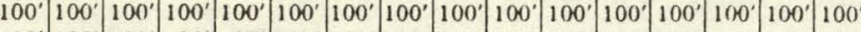

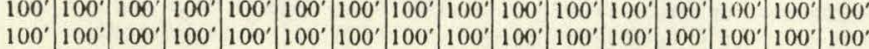
$100^{\prime} 100^{\prime} 100^{\prime} 100^{\prime} 100^{\prime} 100^{\prime} 100^{\prime} 100^{\prime} 100^{\prime} 100^{\prime} 100^{\prime} 100^{\prime} 100^{\prime} 100^{\prime} 100^{\prime} 100^{\prime}$

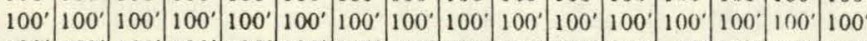

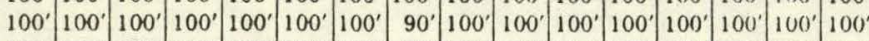
$100^{\prime} 100^{\prime} 100^{\prime} 100^{\prime} 100^{\prime} 100^{\prime} 100^{\prime} \quad 78^{\prime} 100^{\prime} 100^{\prime} 100^{\prime} 100^{\prime} 100^{\prime} 100^{\prime} 100^{\prime} 100^{\prime}$

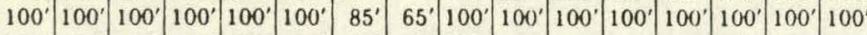

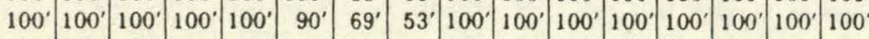

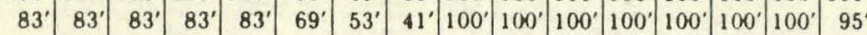

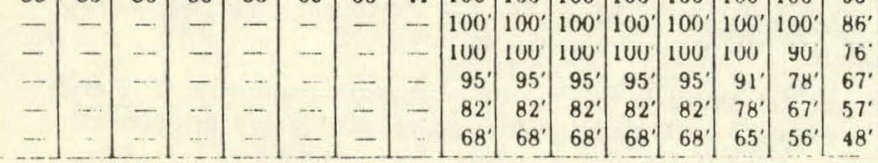

\section{J PUMP}

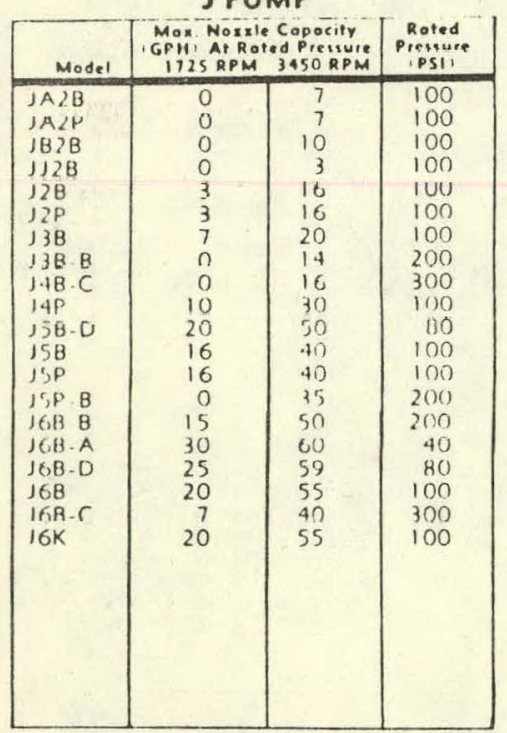

PUMP USAGE IDENTIFICATION

EXAMPLE

\begin{tabular}{|c|c|c|c|c|c|c|c|c|c|c|c|c|c|c|c|c|}
\hline \multirow{2}{*}{$\begin{array}{l}\text { Distance } \\
\text { " ( " Fig. } 4\end{array}$} & \multicolumn{16}{|c|}{$3 /$ "O. I) Tubing (1725 RPM) } \\
\hline & \multicolumn{2}{|c|}{$\mathrm{J}_{2}$} & $J_{3}$ & $J_{4}$ & & $J_{6}$ & $\mathrm{H}_{2}$ & $\mathrm{H}_{3}$ & \multicolumn{2}{|l|}{$\mathrm{H}_{4}$} & $\mathrm{H}_{3}$ & \multirow{2}{*}{$\mathrm{H}_{\mathrm{h}}$} & $\mathrm{H}_{7}$ & \multicolumn{2}{|c|}{$\mathrm{H}$} \\
\hline $0^{\prime}$ & 64 & $4^{\prime}$ & $64^{\prime}$ & $53^{\prime}$ & 41 & & $31^{\prime}$ & $75^{\prime}$ & $75^{\prime}$ & $70^{\prime}$ & & $60^{\circ}$ & & $30^{\prime}$ & & $20^{\prime}$ \\
\hline $1^{\prime}$ & 69 & $9^{\prime}$ & $69^{\prime}$ & & 45 & & $34^{\prime}$ & & $78^{\circ}$ & $72^{\prime}$ & & $62^{\prime}$ & & $31^{\prime}$ & & $21^{\prime}$ \\
\hline $2^{\prime}$ & 74 & $4^{\prime}$ & $74^{\prime}$ & $61^{\prime}$ & 48 & & $36^{\prime}$ & & $80^{\prime}$ & $75^{\prime}$ & & $64^{\prime}$ & & $32^{\prime}$ & & $22^{\prime}$ \\
\hline \multirow{2}{*}{$\begin{array}{l}\text { Distance } \\
\text { (c). Fig } 4\end{array}$} & \multicolumn{16}{|c|}{$3 / \% O$ D. Tubing ( 3450 RPM) } \\
\hline & $\mathrm{J}_{2}$ & $\left|\mathrm{JA}_{4}\right|$ & TJB: & $\bar{s}_{2}$ & $\mathrm{~J}_{3}$ & $\mathrm{~J}_{4}$ & $J_{3}$ & $\mathrm{~J}_{\mathrm{t}}$ & & $\mathrm{H}$ & HB. & $\left.\mathrm{H}_{2}\right]$ & $\mathrm{H}_{3}$ & {$\left[\mathrm{H}_{4}\right]$} & $\mathrm{H}_{3}$ & $\mathrm{H}_{6}$ \\
\hline $0^{\prime}$ & $31^{\prime}$ & $31^{\prime}$ & $31^{\prime}$ & $3 \mu^{\prime}$ & $31^{\prime}$ & $25^{\prime}$ & $19^{\prime}$ & 5 & $25^{\prime}$ & $25^{\circ}$ & $25^{\prime}$ & $25^{\prime}$ & $25^{\prime}$ & $23^{\prime}$ & $20^{\circ}$ & $\overline{7}$ \\
\hline 1 & & $33^{\prime}$ & $33^{\prime}$ & $33^{\prime}$ & $33^{\prime}$ & $27^{\prime}$ & $2 \mathrm{I}^{\prime}$ & & $26^{\prime}$ & $26^{\circ}$ & $26^{\prime}$ & $26^{\prime}$ & $6^{\prime}$ & $25^{\prime}$ & $22^{\prime}$ & . \\
\hline $2^{\prime}$ & & $35^{\prime}$ & $35^{\prime}$ & $35^{\prime}$ & $35^{\prime}$ & $29^{\prime}$ & $23^{\prime}$ & $17^{\prime} \mid$ & 27 & $28^{\prime}$ & $28^{\prime}$ & $28^{\circ}$ & 29 & $26^{\prime}$ & $23^{\prime}$ & $\begin{array}{l}19 \\
19\end{array}$ \\
\hline \multirow{2}{*}{$\begin{array}{l}\text { Distance } \\
\text { "( '“' Fig } 4\end{array}$} & \multicolumn{16}{|c|}{$1 / 2 " O$ D Tubing ( 3450 RPM) } \\
\hline & $\mathrm{JJ}_{2}$ & {$\left[\mathrm{JA}_{4}\right.$} & $\mathrm{JB}$ & $\mathrm{J}$ & $J_{3}$ & J. & $\mathrm{Js}$ & $\mathrm{J}_{\mathrm{h}}$ & JII & $\mathrm{HA}, \mathrm{H}$ & $\mathrm{HB}$ & $\mathrm{H}_{2}$ & $\mathrm{H}_{3}$ & {$\left[\mathrm{H}_{\mathrm{I}}\right]$} & $\mathrm{H}_{\mathrm{i}}$ & $\mathrm{H}_{\mathrm{h}}$ \\
\hline 0 & 1 & $100^{\circ}$ & $100^{\prime}$ & $00^{\prime}$ & $100^{\circ}$ & , & $77^{\prime}$ & $9^{\prime}$ & $100^{\circ}$ & $100^{\circ} 1$ & $100^{\circ}$ & $100^{\prime}$ & $100^{\prime}$ & $94^{\prime}$ & $81^{\prime}$ & 69 \\
\hline & & $100^{\circ}$ & $100^{\prime}$ & $100^{\circ}$ & $100^{\circ}$ & $100^{\circ}$ & $83^{\prime}$ & & $100^{\circ}$ & $100^{\circ} 1$ & $100^{\circ}$ & $100^{\prime}$ & $100^{\prime}$ & $99^{\prime}$ & $85^{\prime}$ & 72 \\
\hline $2^{\prime}$ & $100^{\prime}$ & $100^{\prime}$ & $100^{\prime}$ & $100^{\prime}$ & $100^{\circ}$ & $100^{\prime}$ & $89^{\prime}$ & $68^{\circ}$ & $100^{\prime}$ & $100^{\circ} 1$ & $100^{\prime}$ & $100^{\prime}$ & $100^{\prime}$ & $100^{\circ} \mid$ & $89^{\prime}$ & 76 \\
\hline
\end{tabular}

H PUMP

\begin{tabular}{|c|c|c|c|}
\hline Mndel & 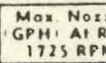 & $\begin{array}{l}\text { Copocily } \\
\text { op prot } \\
3450 \mathrm{RPMM}\end{array}$ & \begin{tabular}{|c} 
Roted \\
Precsure \\
ipsii,
\end{tabular} \\
\hline $\mathrm{HHIB}$ & 0 & 3 & 100 \\
\hline$H A 2 B$ & 0 & $?$ & 100 \\
\hline HAZP & 0 & 1 & 100 \\
\hline$H B 2 P$ & 0 & 10 & 100 \\
\hline$(1+1) B$ & ก & 3 & 100 \\
\hline$H+28$ & 3 & 16 & 100 \\
\hline $\mathrm{H} 2 \mathrm{P}$ & 3 & 16 & 100 \\
\hline $\mathrm{H} 3 \mathrm{BB} B$ & 0 & 14 & 200 \\
\hline H3B & 7 & 2n) & 100 \\
\hline H3B.C & 0 & 7 & 300 \\
\hline Hik & 7 & $7 n$ & 100 \\
\hline $113 \mathrm{P}$ & 7 & 211 & 1100 \\
\hline $113 P$ B & 0 & 1.1 & 200 \\
\hline HiBP IN & () & 1.1 & 300 \\
\hline H.PC C & 0 & ic & $30 n$ \\
\hline HAK N.C & 0 & 22 & 300 \\
\hline H. $+\mathrm{P}$ & 10 & 30 & 100 \\
\hline $\mathrm{H} \rightarrow P \mathrm{~N}-\mathrm{C}$ & 15 & 20 & 300 \\
\hline HSK & .16 & 40 & 100 \\
\hline Hisk N $-C$ & ; & 30 & 300 \\
\hline$H^{\prime}, \mathrm{P}$ & 16 & 40 & 100 \\
\hline $\mathrm{H}, \mathrm{B}, \mathrm{E}$ & 30 & 62 & 20 \\
\hline $\mathrm{HEB} \cdot \mathrm{C}$ & 7 & 40 & 300 \\
\hline$H G K$ & 20 & 55 & 100 \\
\hline HEK C & 7 & 40 & 300 \\
\hline H17K-B & 30 & 80 & 200 \\
\hline & +0 & No & 100 \\
\hline HBK & 80 & No & 100 \\
\hline
\end{tabular}

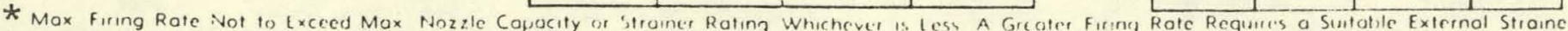




\section{INSTRUCTIONS FOR INSTALLING}

\section{FIELD TYPE M + MG2 BAROMETRIC DRAFT CONTROLS}

This is a dual purpose control.

Single Acting Control - The control is designated as Type $M$ when the stop and two cover plates are in place. This control is for use with oil or solid fuels. Double Acting Control -- The control is designated Type MG2 when the stop and two cover plates are removed. The control is then for use with gas.

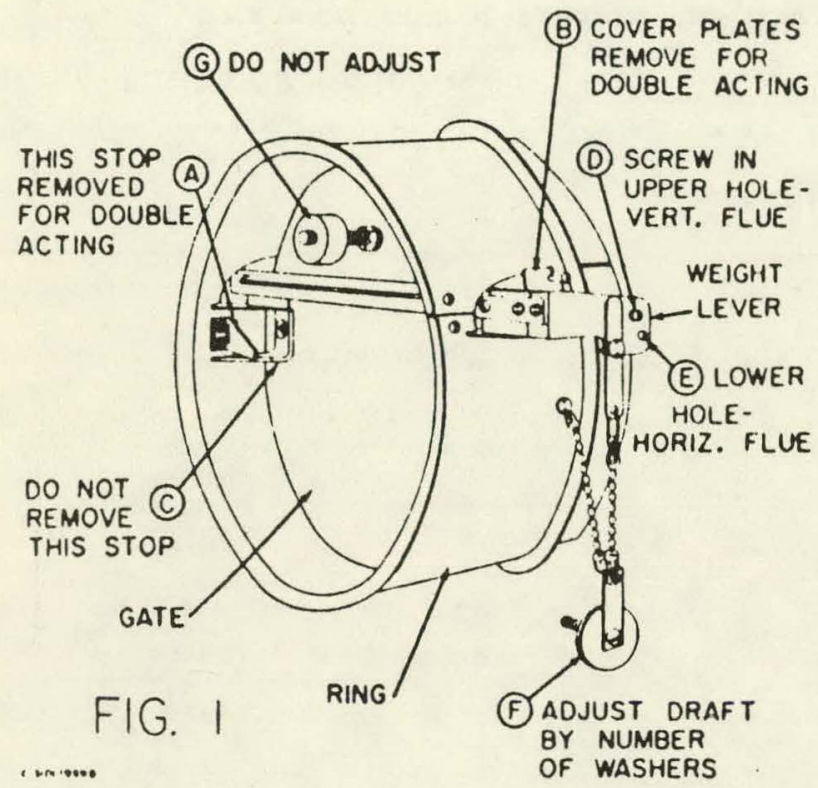

The stop and two cover plates are painted a different color for easy identification. To remove the stop simply remove the two screws that hold it to the ring (Fig. 1 A) To remove the cover plates, (Fig 1 B) bend or break off the tab that goes through the small hole in the ring. DO NOT REMOVE THE STOP ATTACHED TO THE GATE. (Fig. I C)

Installation and Adjustment -- See sections on control locations and collar installation

Insert the draft control inlo the cullus. The fiont lace of the control MUST BE PLUMB. The bearing surlaces MUST BE LEVEL whether the control is on a horizontal, vertical or sloping flue pipe. Use a spint level, plumb and level accurately Secure the control in the collar by tightening the clamping screws. If the collar is supplied locally, the control may be held in place by small bolts or sheet metal screws. If the control has any tendency to sag, support it from the celling by wire or by strapping

Vertical Flues - The control is shupped tor installation in a vertical flue. The screw should be left in the top hole of the weight lever (Fig. I D)

Horizontal Flues - For horizontal flues, remove the screw from the upper hole in the weight lever and insert it in the lower hole. (Fig | E)

Adjusting the Control - The control must be adjusted to the desired draft setting by adding or removing the washer-type weights supported by the two chains. (Fıg. I F) DO NOT MOVE THE LARGE WEIGHTS ATTACHED DIRECTLY TO THE GATE (Fig $\mid \mathrm{G}$ ) as they are used only for adjustment at the lactory.

\section{Control Locations}

For gas fired equipment, the preferred installation is in a bull-head tee (see fig. A, B, C). Fig. D thru J show acceptable locations if it is not leasible to locate the control as per Fig. A, B, C.

Fig. D thru J are the best locations for oil or solid fuel. Locate the control as close as possible to the appliance but at least 12" toward the chimney beyond a stack switch. It must also be located at least 18" from a combustible ceiling or wall.

Do not install in a room separated from the appliance. A void installations in a chimney below where the flue enters unless no other space is available.

When this control is used on a gas-fired unit, consult your local gas company and/or city inspectors to obtain necessary approval betore making the installation. Inspect the heating plant and building for conditions which might cause PROLONGED down drafts and have these conaitions corrected. A chimney with its top lower than other parts of the roof or too close to a higher building may cause prolonged downdrafts. An exhaust fan of sufficient size and location may pull air down the chimney.

\section{BEST LOCATIONS FOR GAS}
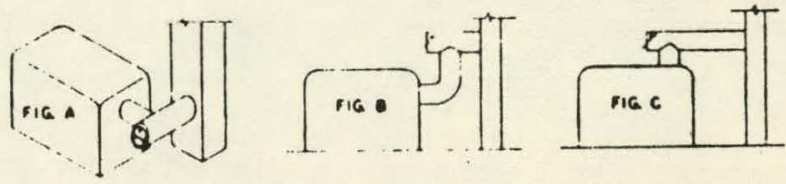

BEST LOCATIONS FOR OIL OR SOLID FUELS
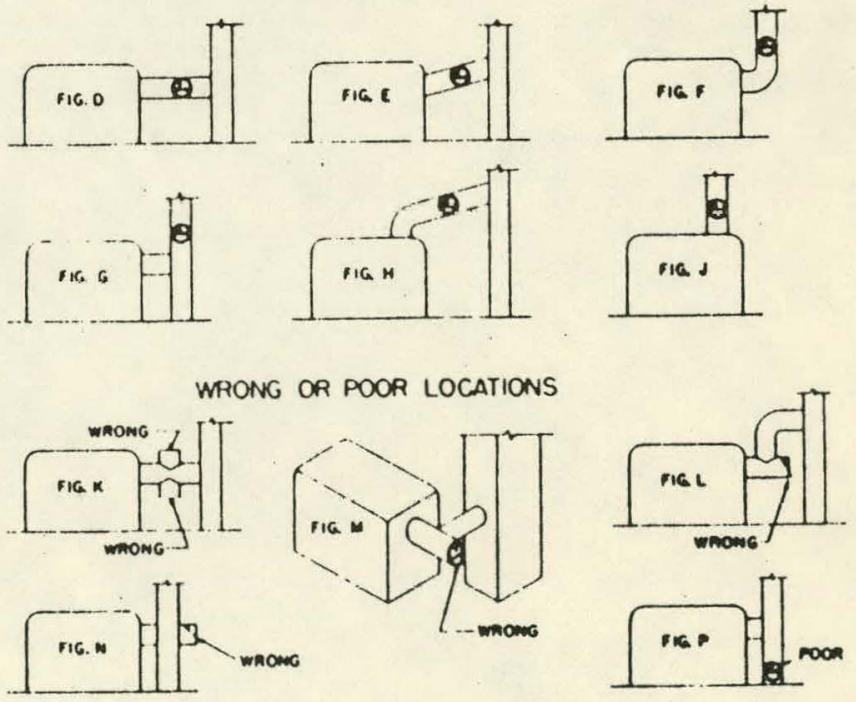

\section{What Draft Setting to Use}

The regulator should be set to maintain as low a draft as will give good combustion. If the desired over-fire draft, taken through a small hole in the combustion 
chamber, is not known, smoke readings, $\mathrm{CO}_{2}$ and fluegas temperature should be taken to determine it the dralt setting is correct on oil equipment. $\mathrm{CO}_{2}$ readings and a check to see that no $\mathrm{CO}$ is present are essential on gas fired equipment.

Gas -.. The usual practice with commercial and indusirial gas fired plants is to adjust for $\mathrm{CO}_{2}$ reudings between $9 \%$ and $10 \%$, or even higher, but this will de. pend upon the analysis of the gas and the type of burner equipment. Consult with the bumer manulacturer

Oil - Forced or induced draft burners can operate with low over-lire drafts. With a burner that depends entirely upon natural draft, the draft in the breeching may need to be guit, hich $\left(.20^{\prime \prime}\right.$ to $\left..50^{\prime \prime}\right)$ to obtain proper overfire curditions. It is essential that $\mathrm{CO}_{\text {. }}$ readings be tuken to determine the proper adjustment.

Coal - With a stoker, the fuel must be of normal thickness, and the stcker running, with its fan adjusted to approximately the correct setting. Drafts over the fire of .08" to .10" (or more) usually are sulficient. Have just enough draft so that no appreciable amount of gas pults back into the roorn through cracks around the fire door at the time that the stoker starts. II there is objectionable smoke, increase drafts slightly. (Consult the stoker manufacturer for proper luel bed and fan adjustment.)

MANOTACTUND 

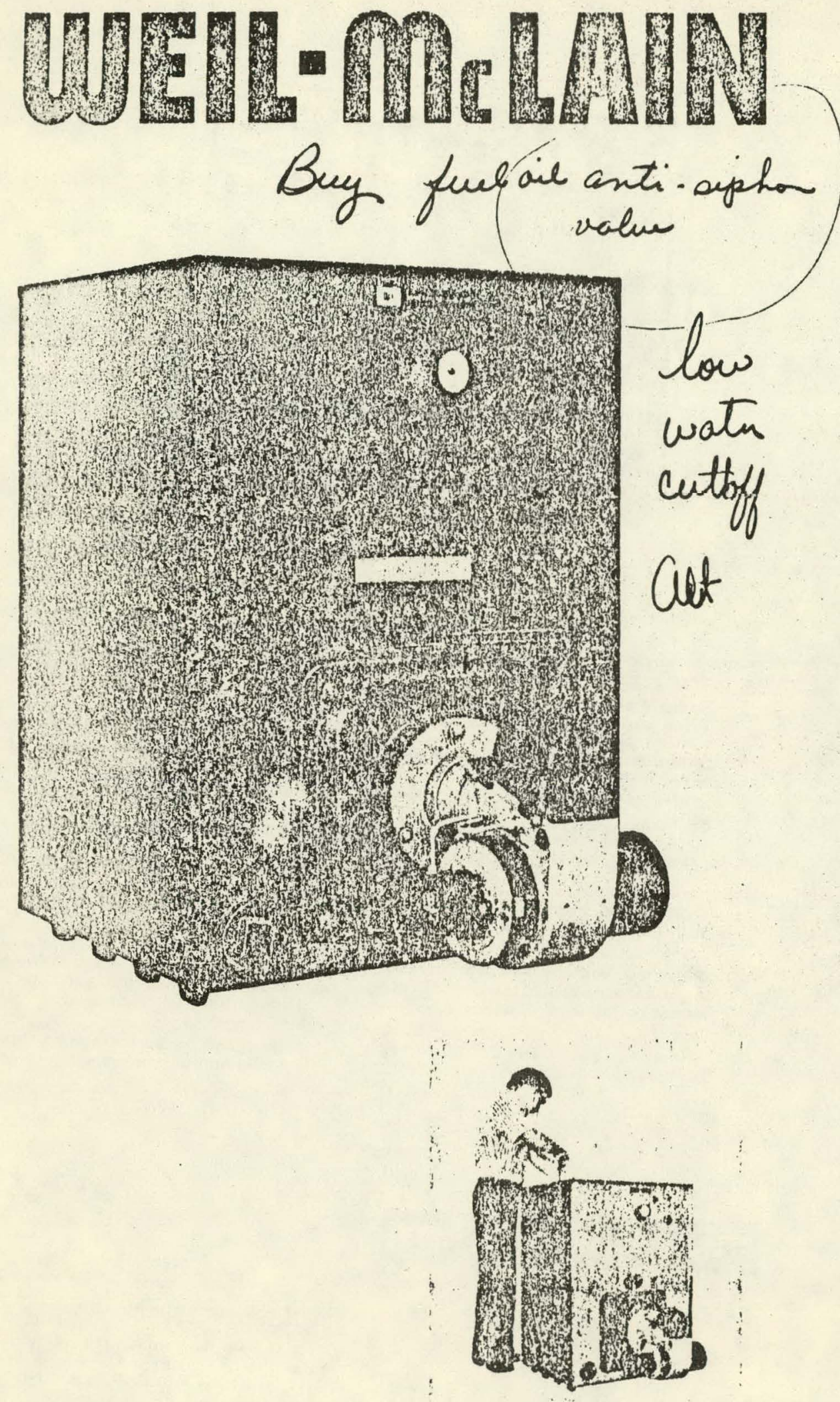

AMERICA'S MOST COMPLETE LINE OF CAST IRON BOILERS RESIDENTIAL ... COMMERCIAL . . INDUSTRIAL . . INSTITUTIONAL 


\section{NO. $0 \begin{gathered}\text { B(IIIEK ANI) } \\ \text { B(NIER.BIIRNER } \\ \text { IINII }\end{gathered}$}

The IV eil. Mlolain No, 76 is available ats

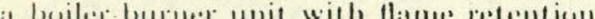

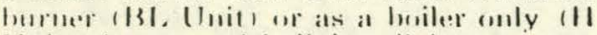
Unit, for nse with light oil hormers ant

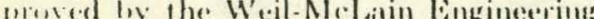
1).partment. Il 76 rallings are apposed by I-13-R when fired withone of the following flame refention burmers.

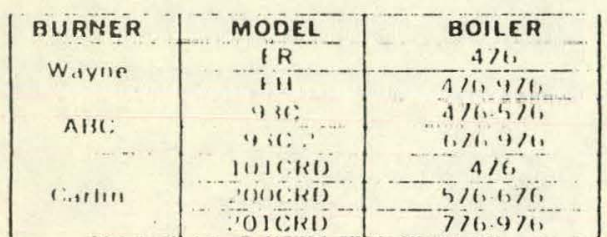

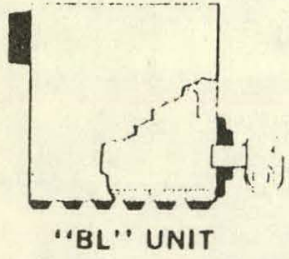

Boiler-Burner Init furnished with flatue refention burner.

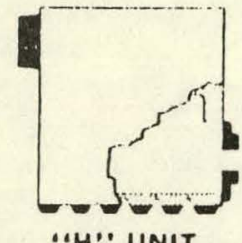

"H" UNIT

Boiler only with inounting plate for aperoved burners.

\section{FACTORY-ASSEMBLED NO 76}

In additun to individual sections, the $N$ (s. 76 Boiler is also available witle sections, hurner mentmeng plate and flue collas farlary-atsembled. Pudividual sectums ats

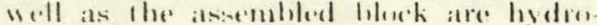

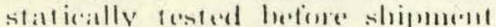

l.ifting lowks atre east on the sides of the frent alld hath sections so the assiombled

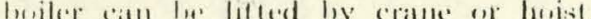

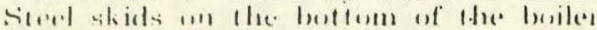
permit mosing the onit with pipe collers.

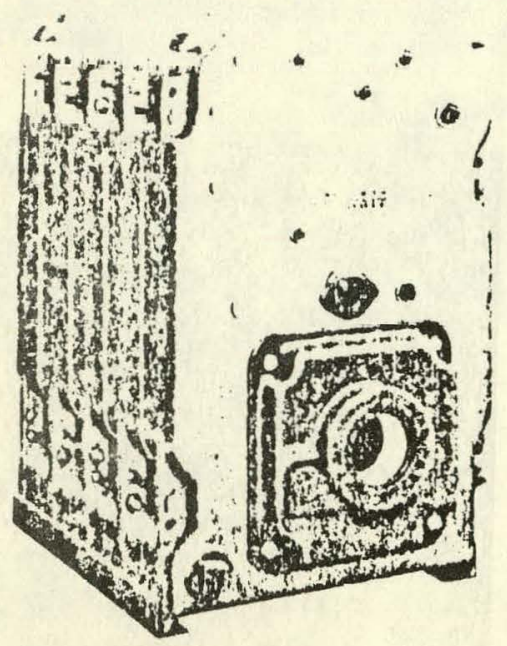

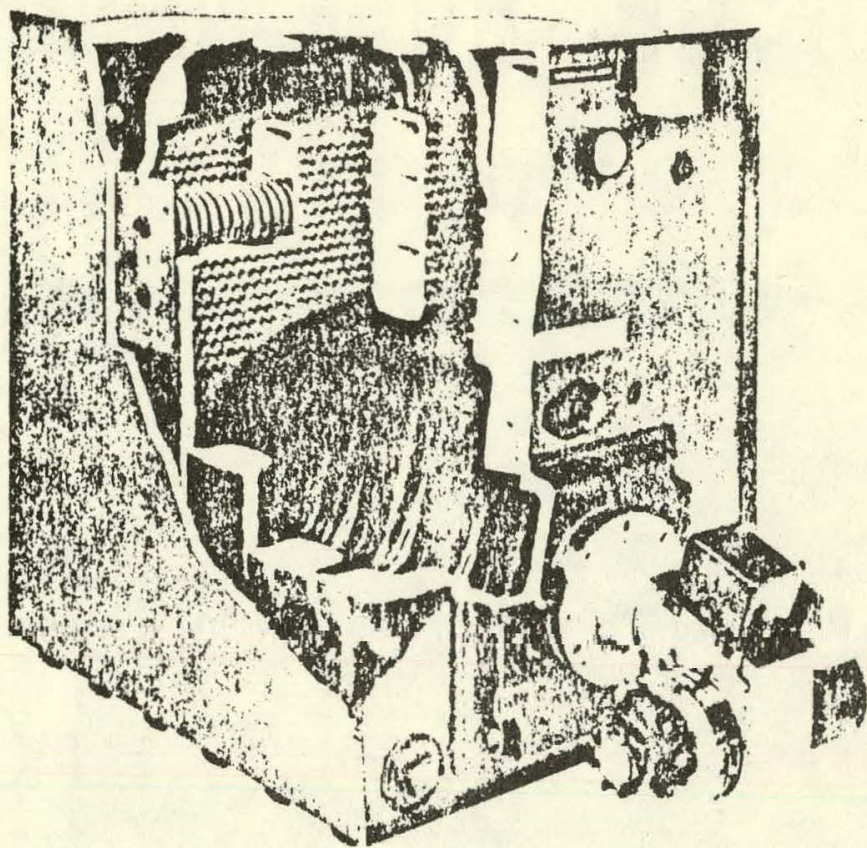

Hydro.Wall design with water circu. lating completely around the com. bustion area... no refractory combustion chamber, no separate base.

Cast iron sections for corrosion re. sistance and extra long life... sealed with asbestos rope.

Avallable in individual sections or with faclory-assembled sections.

Patented section sealing method assures a watertight seal ... re. duces installation time.

Short draw rods for faster assembly and a strain-free boiler.
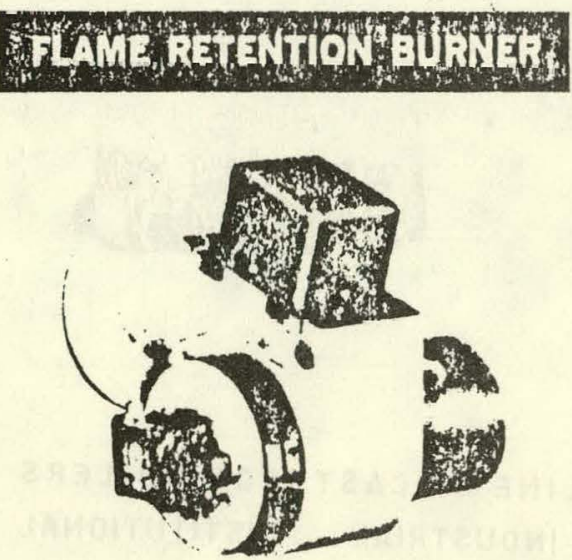

Built-in air eliminator in water boil. ers .... air is diverted to the expansion tank through a 1.Inch tapping located next to the supply outlet.

Multiple tankless heaters. Up to four heaters can be installed on the left side of the lar.gest No. 76 Boiler.

Built-in horizontal flueway elimi. nates the need for a separate sheet metal collector hood.

Steel jacket finished in attractive blue hanimerloid... completel insulated... designed for fasl installation.

Designed for easy cleaning through top cleanout openings.
The BI -76 Boiler IBurner Init is furnished with "Wayme thame retention burner which therenghly mixes the sil with the combustion atir (o) achiese complete comhustionand maximum efficiency. Standard equipment includes a factery-installed and wired potectorelay with cadmium sulfide flane detector.

The lumer mounting plate with refractory which tits around the burner blast tulie, is bolted to the foont section. The horner is positioned on the plate wi three studs. An observation port abo the plate permits clese study of the flanie. 


\section{GENERAL INSTRUCTIONS}

If the boiler was ordered as completely packaged, thoroughly check the boller for any cuncealed damage. If the boiler was ordered as factury assembled or field assembled, open all boxes and check the contents against the packing lists. In the event of shortage or damage, notify the transportation company immediately. As you face the front of the boiler, the side of the boiler to your right will be referred to in these instructions as the Right Hand Side (RH); the side of the boller to your left will be referred to as the Left Hand Side ( $\mathrm{LH}$ ). Boiler(s) must be Installed in accordance with these instructions 80 as not to void our warranty.

\section{AIR SUPPLY FOR BOILER ROOM}

Provisions must be made to supply sufficient air to the boller room at all times for combustion, for ventilation, for operation of the barometric draft regulator (where used), and to prevent less-than-utmospheric air pressure in the boiler room. An opening to the outside with a free cross sectional area of at least 20 square inches for each gallon per hour burner firing rate is recommended (CSA requires $1 \mathrm{sq}$. in. per $5000 \mathrm{~B}$ 'l'UH input). For each 1,000 fect above sea level, increase the fresh air opening by 4 per cent. The boiler room should be isolated from any area served by. exhaust lans. DO NO'T INSTALL AN EXHAUST FAN IN THE BOILER ROOM.

\section{CHIMNEY \\ (also refer to Breeching Erection)}

The No. 76 boiler is designed for natural draft firing. The chimney must be at least of the equivalent ditmeter Indicated on the last page of these instructions under RATING-DATA-DIMENSIONS. On multiple boiler instalations using one chimney, consult Weil-McLain Customer Services Department for additional venting information.

\section{BOILER FOUNDATION}

A boiler fuundation is recummended where the boller room noor is not level or where the boiler room floor can not support the weight of the boiler. Locate the boiler foundation to provide proper clearances around the boiler for installation of the piping, burner, and internal water heater $(\theta)$; ullow a minimum clearance of $22^{n}$ from the back of the foundation for breeching

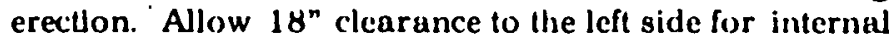
heaters if used. A level concrete pad or curb foundation is suggested of the size shown in the chart and Figure 1.

\begin{tabular}{|c|c||c|c|}
\hline \multicolumn{4}{|c|}{ BOILER FOUNDATION SIZES } \\
\hline BOILER NO. & $\iota$ & BOLER NO. & $t$ \\
\hline 476 & $24^{\prime \prime}$ & 776 & $42^{\prime \prime}$ \\
\hline 576 & $30^{\prime \prime}$ & 876 & $48^{\prime \prime}$ \\
\hline 676 & $36^{\prime \prime}$ & 976 & $54^{\prime \prime}$ \\
\hline
\end{tabular}

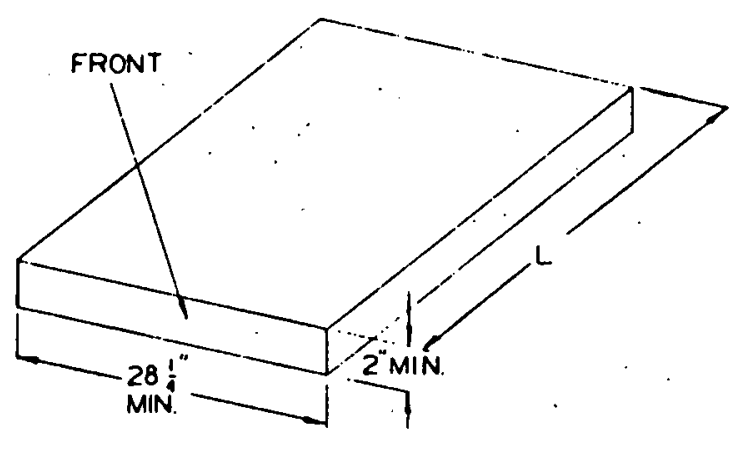

FIGURE 1

If the boiler was ordered as completely packaged or with the sections factory assemibled, locate the unit on the boiler foundation or on the boiler room floor according to the separate instructions furnished and liigure 1. After the boiler has been positioned in the selected location, proceed to "Hydrostatic Pressure Test of Boiler".

\section{ASBESTOS ROPE}

Listed in the Asbestos Rope Usage Table are the places usbestos rope must be used and the diameter and length of each piece. Asbestos rope must be used where indicated. For expediency, the asbestos rope can be precut prior to starting the section assembly.

\begin{tabular}{|c|c|c|}
\hline \multicolumn{3}{|c|}{ ASBESIOS ROPE USAGE TABLE } \\
\hline $\begin{array}{l}\text {-NOMINAL } \\
\text { DIAMEIER OF } \\
\text { ASBESIOS ROPE }\end{array}$ & $\begin{array}{l}\text { CUT } \\
\text { IENGTHS }\end{array}$ & ROPE USAGE AND LOCATION \\
\hline $\begin{array}{c}\text { 3/8" } \\
\text { Stranded }\end{array}$ & $\begin{array}{l}26^{\prime \prime} \\
98^{\prime \prime} \\
42^{\prime \prime} \\
58^{\prime \prime}\end{array}$ & $\begin{array}{l}\text { Cleanout Plates } \\
\text { Perineter of each Section } \\
\text { Drafthood Coltar } \\
\text { Burner Mounting Plate }\end{array}$ \\
\hline $1 / 4^{\prime \prime}$ & $12 "$ & $\begin{array}{l}\text { Observation Port Frarnes } \\
\text { (Front and Back) }\end{array}$ \\
\hline
\end{tabular}

"Approximate diuineter al uncompressed $1 / 4^{n}$ nomlnal diameler rope is $5 / 16 "$ $3 / 8^{\prime \prime}$ strunded rope is $/ 1 / 6^{\prime \prime}$

\section{SECTION ASSEMBLY}

1. I'repare the back section for erecting the boiler. a. Lay bark section on floor with ports facing up. Apply water-glass, as an adhesive, to the target wall area (i.e., back end of firebox) of the section. Press nexible refractory blanket into position.

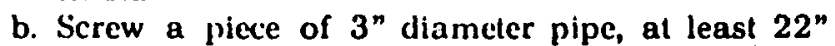
long, into the $3^{\prime \prime}$ return tapping in the back section.

c. Locate the back section on the boiler foundation shown in ligure 1 . Hlock under the $3^{n}$ plpe to hold the buck section up right without additional support. 'These sections ure top-heavy and will not stand individually without support. Make sure that the section remains in plunib. The $3^{\text {n }}$ pipe can be removed after several sections have been erected. 
2. Make a small continuous bead of "Asbestos Rope Adhesive" in the bottom of the curved sealing grooves located around the perimeter of the section. Sinooth out the adhesive using the brush provided.

3. Position the $3 / 8^{\prime \prime}$ by $98^{\prime \prime}$ long asbestos rope on the section by starting at the cleanout opening (Sec Figure 2). Be sure the asbestos rope is well seated at all points in the sealing grooves so that a gas-tight senl will be maintained between the sections. DO NOT APPLY adhesive to the opposing sealing grooves of the next boiler section.

4. Wipe the port openings with a clean rag to remove any grit from the machined surfaces. Dn not use any cleaner that contains a petroleum base distillate (oil) (1) clean ports. I']ace the nominal $7-1 / 2^{n}$ and nominal $3-1 / 2^{n}$ elastomer sealing rings in the appropriate port openings (see Figure 2).

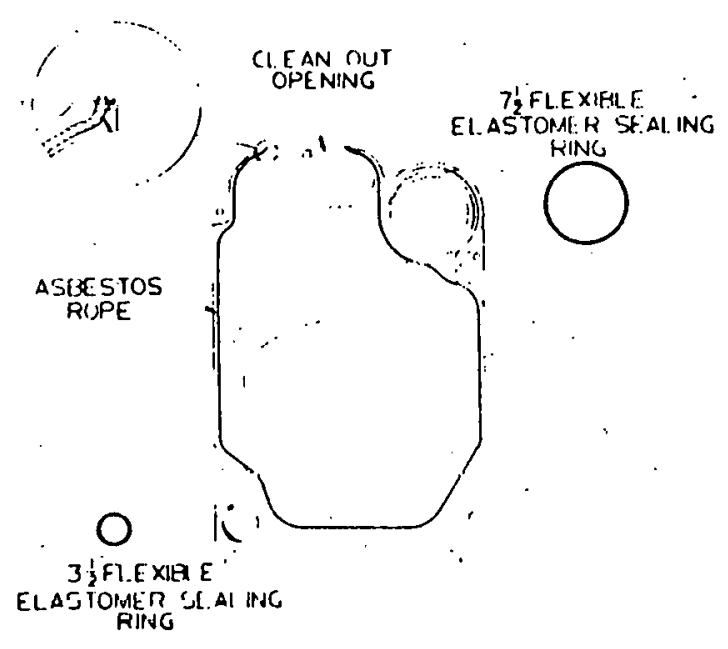

FIGURE 2

5. Prepare the port openings in an intermediate section. "The "lI" Intermediate sertions (if used) must be installed in the order given in the Sextion Arrangenent 'Table With Indirext Water Heaters. Note that 18" cleurance must be provided on the left side for heater inslullation.

6. Discard the $3 / 8$ " diameler rods which are required only during shipment; Hese rods must nest be used to draw the sections logether.

a. Position the intermediate section (see figure 3). ()il the threads of four (4) of the draw rods (5/8" $\left.\times 9^{n}\right)$ and slip) (hem through the lugs on the top and sides of each section. l'lace at washer conly one washer per draw rod) under each nut which is to be lightened, with a drop of oil between washer and nut.

b. Sturting with the draw rods nearest the port openings, draw the sections together muformly until the metal around the ports touch and the pucls at the opposite comers touch. W'hen properly pulled tegether the gaip around the port openings should be less than 0.032". Check with a feeler gauge.

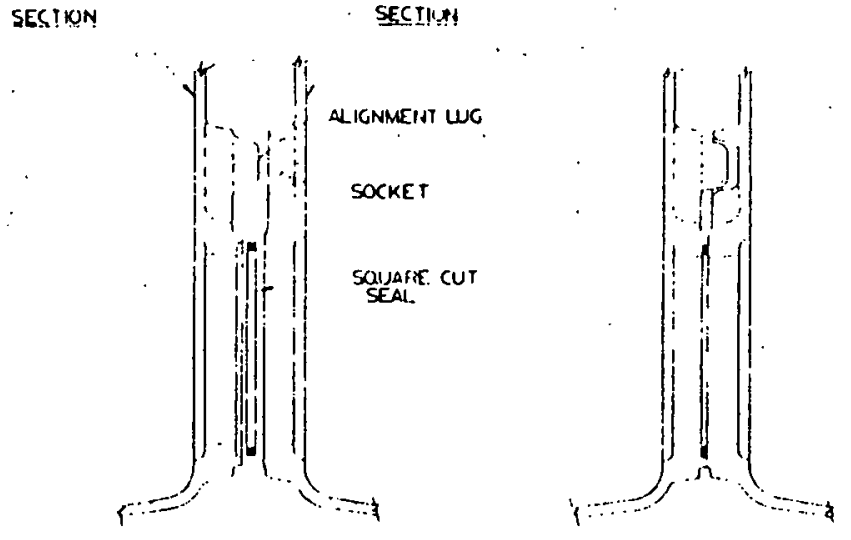

FIGURE 3

c. 1)o not "back off" the draw rod nuts.

7. Set the remaining boiler sertions into position with the "Il" sections placed (If used) in the proper order given in the Stxtion Arrangement 'lable. Check the asbestos rone seal of each section before proceeding to the next section; the boiler must be sealed gas-tight.

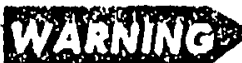

Ise of chemical cleuners or sealants in any Weil-McLnin boiler is not recommended. IN PAKTICUI,AK, PHODUC'TS CONTAINING PETROLEUM DISTILLATES MUST NEVER BE USED IN TYPE 76 BOILFIRSI

8. Four $1 / 2^{n} \times 4-1 / 4 "$ studs are provided to secure the Jumer Nounting I'late to the front section (ese liiguro (1).

a. "lhread two $1 / 2$ " nuts on the rounded end of a 1/2" $\times$ 4-1/1" stud locking them together, and thread the flatl end of the stud inte one of the four tapped loles located around the burner Mlumbting, llate opening in the front section.

b. Remove the nuts from the stud.

c. Repeat sitept "a" and "b" fur the romainims three studs.

d. Hanfs one Refractory Retainer (stainless steel plate) overe eath mounting slud.

9. Ising Astrestos Rope Arlhesive in the groove around the Bumer Nounting Mate opening in the fromt section, position the $: 3 / 8$ " diameter by $58^{n}$ long rope in the froove making sure the ends overlap, and install the Burler Mlomiling lyate using the 1/2" washers and nuts: provided. Nake sure Burner Plate is installed with the round secondary air opening to the left (sce ligure 4).

16. Ilsing Asbestos Rope Arlhesive, position the 1/4" diancter by 12 " long Asbestes Rope in the sealing growve making sure the ends overlap at least 1 ". Install the froml (Hsicration l'ort Assembly using the mumber $10-32 \times 1$ "Truss head screws as illustrated in ligure 4. 
SECTION ARRANGEMENT TABLE WITH INDIRECT WATER HEATERS

\begin{tabular}{|c|c|c|}
\hline BOILER NO. & $\begin{array}{c}\text { MaXIMUM NO. } \\
\text { OF HEAIERS }\end{array}$ & $\begin{array}{l}\text { SECTION ASSEMBIY } \\
\text { ALI HEATERS MUST BE ON IEFT SIOE OF BOILER }\end{array}$ \\
\hline $476 W \& S$ & 1 & F.TI.I.B \\
\hline $576 W \& S$ & 2 & F.TI-I.TI.B \\
\hline $676 W \& S$ & 2 & F.TI.I.TI.I.B \\
\hline $776 W \& S$ & 3 & F.TI.I.*.I.TI.B \\
\hline $876 W \& S$ & 3 & F.TI.I.TI-I.I.TI-B \\
\hline $976 W \& S$ & 4 & F.TI.I.TI.I.*.1.TI:H \\
\hline
\end{tabular}

F = Front Section; B - Back Section; 1 = Intermediate Section; TI = Intermediate Section with Tankless Heater Opening

* A Tl section can be located in this position instead of regular intermediate section. but installer must cut jacket side panel to accomniodate heater openning . no knockout is provided.

11. If the boiler was ordered with "TI" intermediate section(s), install the indirect water heater(s) or heater opening cover plate(s) using the gasket(s), 3/8"- 16 $x$ 3/4" Hex Head Screws and washers as shown in Figure 4. If the water heater piping is installed as shown in Figure $x x$, the jacket can be erected either before or after the plping.

12. Using Asbestos Rope Adhesive, position the 3/8" $x$ 26" long Asbestos Kope around the inside perimeter of the cleanout plates. Mount the cleanout plates over the openings as shown in Figure 4.

13. Using Asbestos Rope Adhesive, position the $3 / 8^{\text {n }}$ diameter by $42^{\text {" }}$ long asbestos rope in the groove on the Draft llood Collar making sure the ends of the rope overlap at least 1 ".

14. Secure the Draft llood Collar to the back section using the 3/8" $\times 1$ " Hex Head Cap Screws and Washers as illustrated in Figure 4.

\section{HYDROSTATIC PRESSURE TEST OF BOILER}

1. Secure a drain cock (not supplied) to the $3 / 4$ " drain tapping.

2. Install a water pressure gauge in one of the boiler tappings.

3. lustall a hleed valve in boiler tapping $K$ to vent air as the boiler is filled.

4. Ilug all remaing boiler tappings. Refer to the Control T'apping 'lable.

5. liill the hoiler with water and completely vent all air. 'Test the bojler with water pressure at $\mathbf{4 5}$ pounds per square inch.

6. 'lhoroughly inspect the entire boiler for water leaks.
7. 1)rain the boiler and remove plugs from those tappings which will be used. Refer to the Control Tapping 'Table.

\section{SUPPLY AND RETURN PIPING}

It is recommended that the system supply and return piping be installed and the piping connections attached (1) the boiler beforc erecting the Jacket or installing the controls to avoid any possible damage to the Jacket or Controls. Recommended piping arrangements for No. 76 water and steam boilers are shown in Figures 5,6 and 7 and the minimum recommended pipe sizes are listed for each piping arrangenient. The supply and return piping will not interfere with the erection of the builer jucked.

\section{WATER BOILER SUGGESTED PIPING CONNECTIONS}

Figure 5 and the accompanying chart show the recommended piping connections and minimum recommended pipe sizes for No. 76 water boilers. In most cases, it is advisable to pump water away from the boiler by comnecting the supply piping, as illustrated in Figure 5, to the suclion of inlet side of the circulator.

In sizing the supply and return piping, start with the minimum recommended pipe size and proceed at fill diameter for 10 times that diameter before making any reduction. An example of this would be a 3 " retuin would not be reduced any closer to the boiler return tapping thim 30". Horizonlal expansion tank piping must pitch upward at least 1 " for each 5 feet of piping from the boiler to the tank. Whiere system 


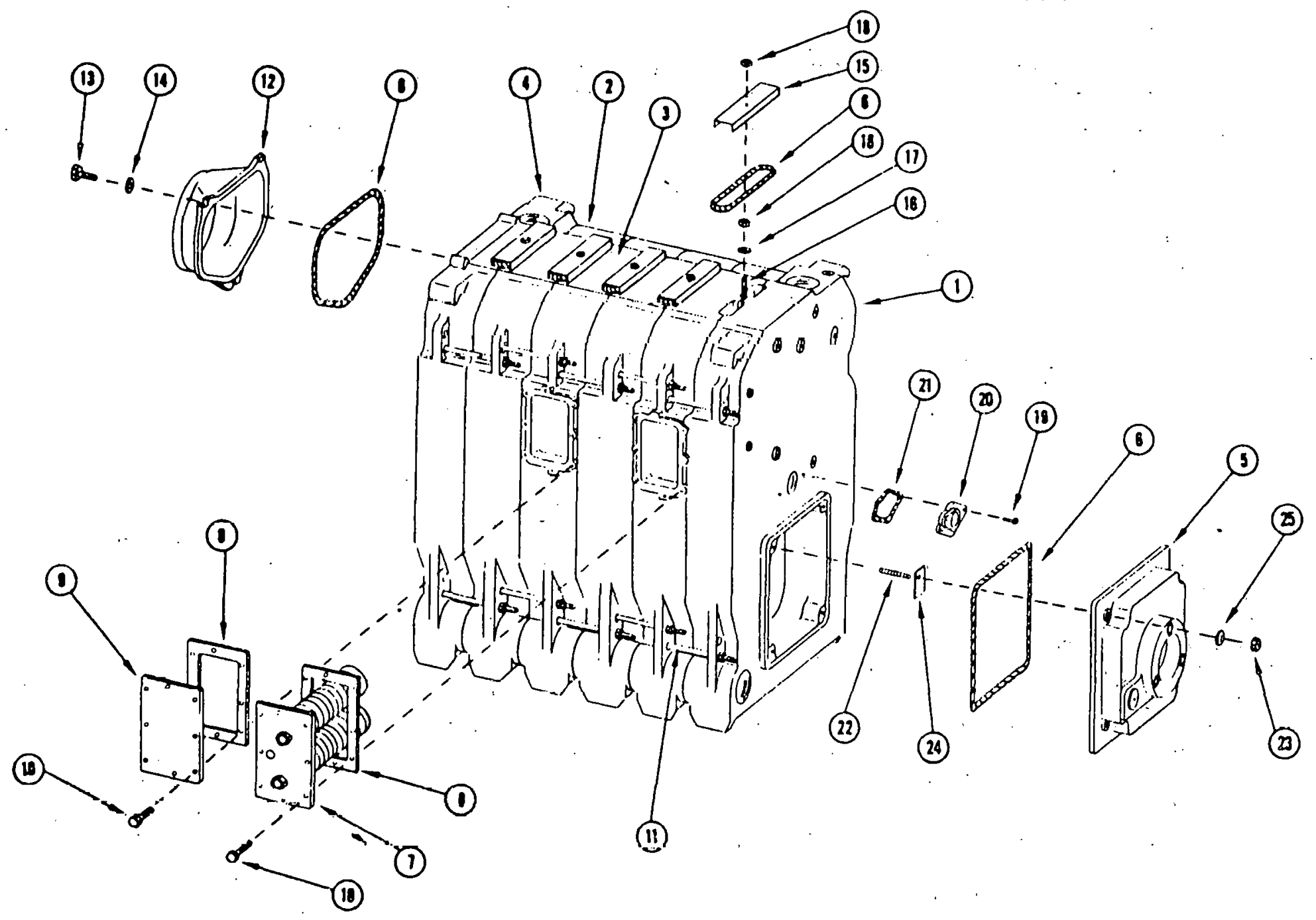

FIGURE 4

1 roint Section

2. Regular Intermediate Section

3 Tankless Intermediate Section

4. Back Section

5 Burrie Múvinting Flate

6. Asuestos Rope, 3/8" Stranded

7. Tankless Heater

8. Tankless Heater Gasket

9. Heater Cover Plate
10. Cus 3uew, 3/8" - 16:3/4"

11. Draw Rod, 5.8" $\times 9 "$

12. Drall Heod Collar

13. Cap Sirew. 3:4" $-16 \times 1 "$

I1. Wastile, $3 ; y^{\prime \prime}$

15. Cleanout Plate

16. Carriage Bolt, $1 / 4 "-20 \times 1 \cdot 1 / 2 "$

17. Washer, 1/4"
18. Nut, Her, $1: 1 " 20$

19. Sciew, S.IP Type F Pan Hd.

20. Observation Port Assembly

21. Asbestos Rope, 1/4" Stranded

22. 3tuu!, T, Triil, 1/?", \& $4 \cdot 1 / 4$ "

23. Nut, Hex, 1/2"

24. Retainer, Front Refractury

25. Washer, 1/2" lemperature modulation is achicved by means of threen'aly valves, care must be exercised in piping the system (1) protect the boiler from thermal shock which rould result from returning room temperature waler at high velocities to the hest beiler. Where three-way valves are empleyed, consult Wedil-Nelatin (instomer Services l)e partment for piping recommendations; primaryseromdary pumping is pretorsed. 


\section{RECOMMENDED PIPING CONNECTIONS FOR WATER BOILERS}

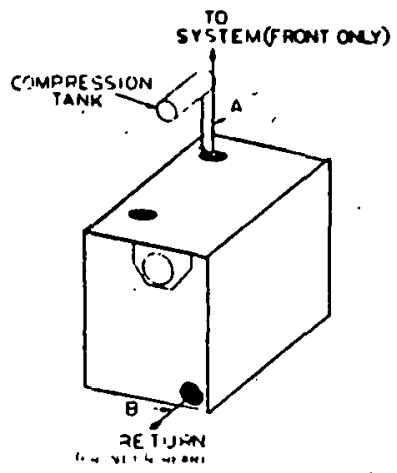

FIGURE 5

WATER BOILER PIPING RECOMMENDED MINIMUM PIPE SIZES

TABLE I

FOR KNOWN FLOW RATES

\begin{tabular}{|c|c|c|}
\hline $\begin{array}{c}\text { WAIER } \\
\text { FIOW RATE } \\
\text { GPM }\end{array}$ & $\begin{array}{c}\text { SUPPLY } \\
\text { PIPE SRE } \\
\text { A }\end{array}$ & $\begin{array}{c}\text { REIURN } \\
\text { PIPE SRE } \\
\text { B }\end{array}$ \\
\hline To 9 GPM & $1^{\prime \prime}$ & $1 "$ \\
\hline $10 \cdot 16 \mathrm{GPM}$ & $1^{1 / 4^{\prime \prime}}$ & $1{ }^{\prime \prime}$ \\
\hline $17 \cdot 21 \mathrm{GPM}$ & $11 / 2^{\prime \prime}$ & $112^{\prime \prime}$ \\
\hline $22 \cdot 35 \mathrm{GPM}$ & $2^{\prime \prime}$ & $2^{\prime \prime}$ \\
\hline $36 \cdot 50 \mathrm{GPM}$ & $2^{1 / 2 "}$ & $2^{11 / 2}$ \\
\hline $51 \cdot 76 \mathrm{GPM}$ & $3^{\prime \prime}$ & $3^{\prime \prime}$ \\
\hline
\end{tabular}

- High lemperoture rize through boller is permissible when boilor piping con. nections ore sized using above Iable I. INIERMITIENI flow of HIGH velocitios moy domage ony bailes.

TABLE \|

FOR UNKNOWN FLOW RATES**

\begin{tabular}{|c|c|c|}
\hline $\begin{array}{c}\text { BOILER } \\
\text { NUMBER }\end{array}$ & $\begin{array}{c}\text { SUPPYY } \\
\text { PIPE SRE } \\
\text { A }\end{array}$ & $\begin{array}{c}\text { REIURN } \\
\text { PPE SRE } \\
\text { B }\end{array}$ \\
\hline 476 and 576 & $2^{\prime \prime}$ & $2^{\prime \prime}$ \\
\hline 676 and 776 & $212^{\prime \prime}$ & $21,2^{\prime \prime}$ \\
\hline 876 and 976 & $3^{\prime \prime}$ & $3^{\prime \prime}$ \\
\hline
\end{tabular}

-All piping sizes are based upon 20 deg. F. lemperglule rise through the boiler. For use of other lemperature rises through the boiler (i.e. higher flow rutes) determine the flow role and use Tuble I for pipe sites.

\section{STEAM BOILER PIPING AND HEADERS}

Figures 6 and 7 and the accompanying chart show recomnended piping comnetions and minimum reccommended pipe sizes for No. 76 steam boilers. 'The supply and return steam piping is not supplied with No. 76 steam boilers but shomld be installed as illustrated. Controls (safety valve, low water cut-off, gallge glass, etc. ) are not shown on the steam piping diagrams in order to show more clcarly the steam piping and Hartford l.oop.
WALNTS

The satisfactory operation of any steam heating boiler: depends upon adequate return of condensate to the boiler to muintain a steady water level. In rambling buildings with extended systcm piping, nuisance shuidowns sometines result when the condensate returning from the system lags behind the evaporation capacity of the boiler. 'To maintain a steady water line, avoid the introduction of excessive amounts of raw make-up water, und to prevent nuisance shut-downs due to a tenmorary low water level, it is recomnended that a low water cutuff and pump control, condensate receiver, and condensate boiler feed punp be installed. Consult Wcil-Mclaain Customer Services lepartment for. application infurmation.

\section{RECOMMENDED PIPING CONNECTIONS FOR STEAM BOILERS}
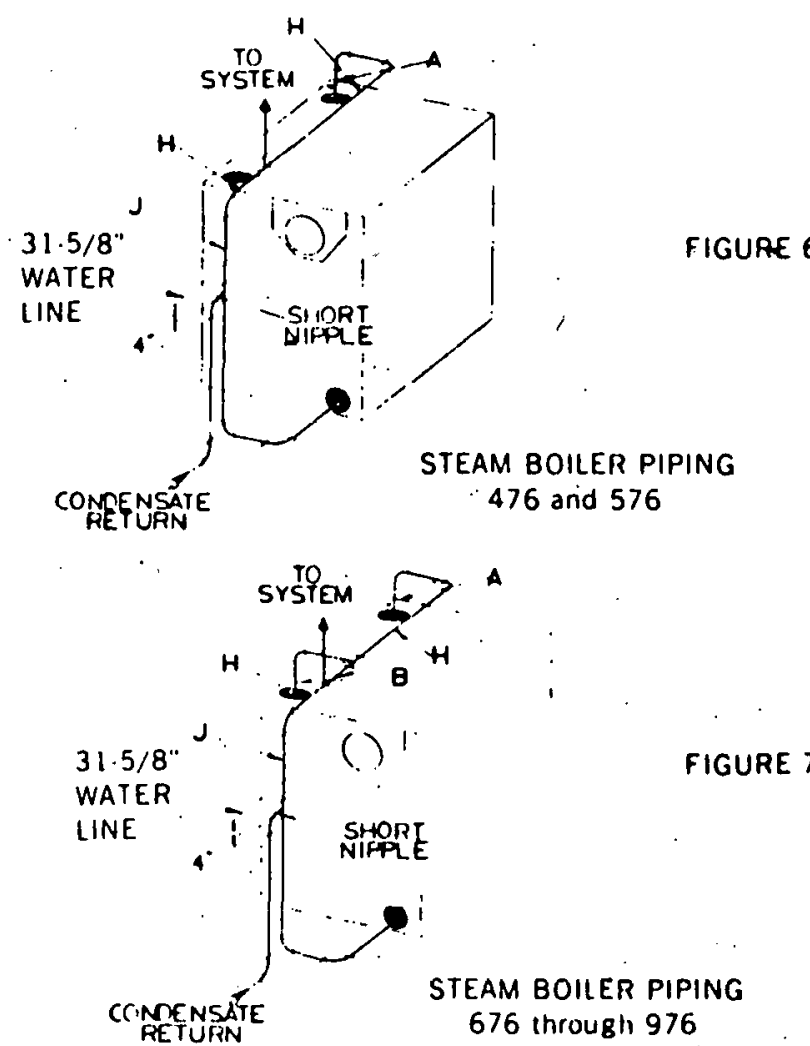

STEAM BOILER PIPING

MINIMUM RECOMMENDED PIPE SIZES

\begin{tabular}{|c|c|c|c|c|c|}
\hline \multirow{2}{*}{$\begin{array}{l}\text { Fig. } \\
\text { No. }\end{array}$} & \multirow[b]{2}{*}{ Boiler Sixe } & \multicolumn{2}{|c|}{$\begin{array}{l}\text { Piser } \\
\text { Pipe Size }\end{array}$} & \multirow{2}{*}{$\begin{array}{c}\text { Meoder } \\
\quad \mathrm{H} \\
\end{array}$} & \multirow{2}{*}{$\begin{array}{c}\text { Equaliaer } \\
1\end{array}$} \\
\hline & & A & - & & \\
\hline 6 & 476 & 3" & & 3" & $11 / 2^{\prime \prime}$ \\
\hline 6 & 576 & 3" & & 3" & $11 / 2 "$ \\
\hline 7 & 676 & $21 / 2 "$ & $21 / 2 "$ & $4^{\prime \prime}$ & $11 / 2 "$ \\
\hline 7 & 776 & $21 / 2^{\prime \prime}$ & $2^{1 / 2 "}$ & $4 "$ & $11 / 2^{\prime \prime}$ \\
\hline 7 & 876 & 3" & $3 "$ & $1 "$ & $2^{\prime \prime}$ \\
\hline 7 & 976 & 3. & $3^{\prime \prime}$ & $4^{\prime \prime}$ & 2" \\
\hline
\end{tabular}

-24" Minimuin from wolerline 10 hooder 


\section{ATTACHING THE JACKET}

The boiler should be pressure tested before the jacket is erected. Follow the Jacket krecting Instructions parked in the jacket carton which contains the front and Back Jacket l'anels for jacket installation procedures.

\section{TANKLESS HEATER HOOK-UP}

Where the boiler was ordered with (a) tankless heater(s) it is recommended that the piping to and froin the heater be sized no smaller than the heater inlet and outlet piping connections. 'The tankless heater piping should be installed as illustrated in ligure 8 . It is recommended that a flow liegulating valve be Instulled in the cold water supply piping to each heater. Fach flow Regulating Valve should be sized according (1) the intermittent draw rating of each tankless heater. Do not pipe multiple indirect waler heaters in scries. An Automatic Mixing Valve may be installed in the domestic hot water supply piping from the heater(s) to permit regulation of the domestic. hot water temperature. Install the operating control in the control lapping in the heater plate. In hard water areas, it is advisuble to boften the cold water to the healer(s).

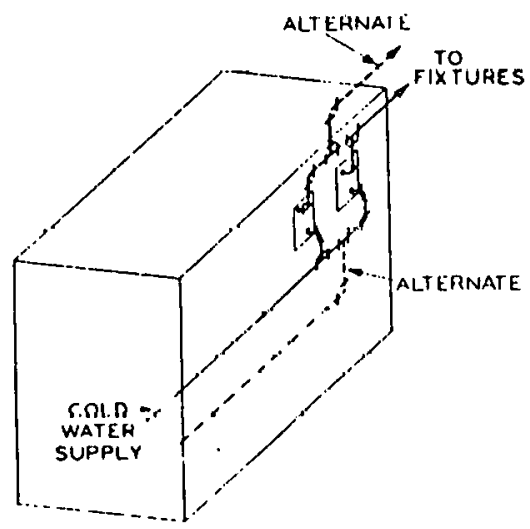

FICIURE B

\section{WTANTHO INSTALL BOILER CONTROLS}

\section{Steam Boiler:}

1. Install the stian Safety Valve in the proper tapping as indicated in the control Titpping 'Table. The sarety' valve outlet slowald be piped to a hour drain or near to the floor. Do not pipe the salfty valve discharge to any area where freceing temperatures could occur.

2. A low water cutolf must be installed on this boiler to meet AsMlic code requirements.

3. Where an optionitl 1/2" low water cut-off is employed, install the control in the gange glass tappings (using the quick connections furnished with the low watter cut-off in accordance with the comlrol lapping liable.

4. Where an optional 1" low witler cut-off, combination low water cut-off and fecder, or combination low water cut-off and pump control is employed, install

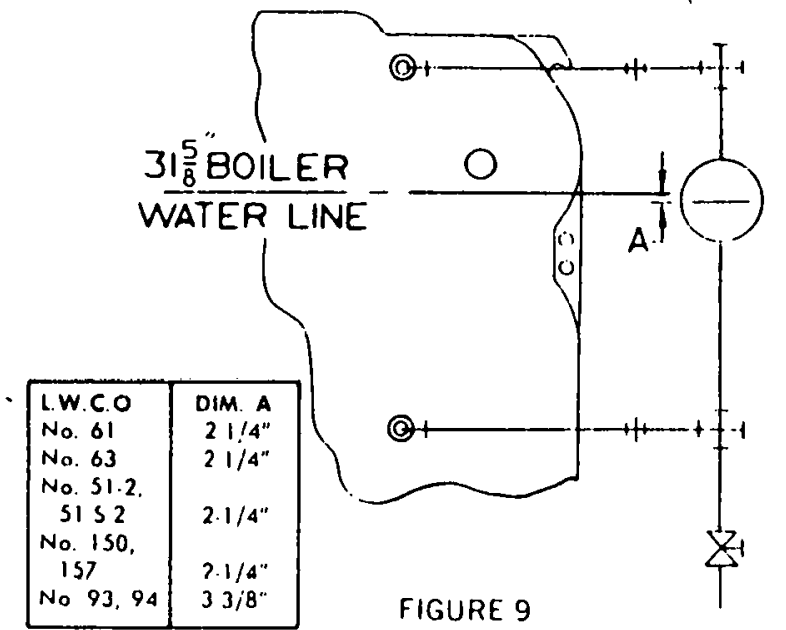

Noto: The schematic heok up stiown provides for the proper locution of the low waslet cut oll level. feeder operaling level, ar punp coutrol operating level cully where " single curirol is applied to the boller. Where mulisple conirols ore to be applied lsuch us " primary low woter cul-ufl and a soparote low wister cul all urid leciler combincilion, or a sepurate low water cutalt and pump coilloll. The primury low wuler culdoll operuting level must bo sel lower than the leeder or pumy curliol operaling levels. Refer to the sepurate manutoc. lurer's insleuclions or contact Weil.Mclain Cusluner Servires Deparlment lor localing the leeder or pump cuntral operaling levels in relation to the primary low water cut ofl operating level

the control in accordance with the Control Tapping lable using fitlings (not lurnished). If a low vater cul-off is to be used that is not dimensionally diagrammed in Figure 9, locate the cast-on body mark of the control 2" below the nurmal water line.

5. Install the (iange Chass ('ocks, Water Gauge Glass (iuards in accordance with the control thapping 'Table.

(i. Linstall the sllam Pressure (pherating and Pressure timit controls and the l'ressure Gauge in the proper tapping as indicated in the Control tapping lable using the l'igtuil syphon and fillings furnished as il. lustrated in rigure 10.

7. Install any additional or optional stean boiler contruls atcosding to the component manufacturer's instructions furnished with the control.

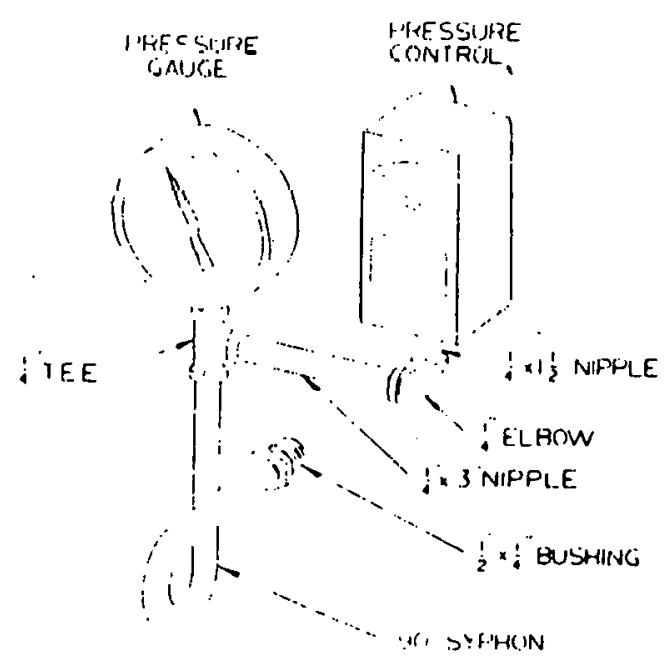

FIGIJRE 10 


\section{Water Boiler:}

1. Install the Pressure Relief Vulve In the proper tapping as indicated in the Control Trupping 'luble. The relief valve outlet should be piped to a four drain or near to the noor. Do not pipe the relief valve discharge to any area where freezing temperalures could occur.

2. Install the Pressure-Temperature Gauge as indicated In the Control Tapping Table.

3. Where an optional 1" low water cut-off is employed, install the control in accordance with the control Tapping Table using the fittings (not furnished) as illustrated in ligure 9. If a low water cutouf is to be used that is not dimensionally diagrammed in Figure 9 refer to the separate manufacturer's instructions for locating the control.

4. Install the Combination limit Cuntrol as indicated in the Control 'Tapping 'Table.

5. Install any additional I.imit controls in the proper tappings as indicuted in the Control lapping 'Table.

6. Install any additional or optional water boiler controls according to the component manufacturer's instructions furnished with the control.

\section{HiNIS \\ BREECHING ERECTION (also refer to Chimney)}

Long horizontal breechings, excessive numbers of elbows or tees or other obstructions which are restrictive to the flow of combustion gases should be avoided.

Fit a plece of full sized heavy gange steel breech. ing (same diameter. as Draft Hood Collar) over the Draft Hood Collar and connect to chimney.

\section{BURNER INSTALLATION}

Carefully unpack the burner from its shipping container and check the contents. In case of shorlage or damage, notify the transportation company immediately. The envelope of papers enclosed with the burner is to be used, preserved, and turned over to the owner and/or the owner's representative.

secure the Burner Mlounting Flange to the Bumer Mounting l'late using the bolts provided until a rigid installation is accomplished. A gas-tight seal must be maintained between the burner mounting flange and the burner mounting plate or damage to the burner air tube will result.

\section{WARNINO WIRING THE BOILER}

All wiring should be installed in compliance with the rules of the National lilectrical Code, for installation in the II.S.A. or Canalian lilectrical Code C22.2 l'art 1 surance requirements or codes having jurisdiclion. Op. erating and safety circuit wiring may be No. 14 gallge wire. l'owe' supply wiring to the burner shall be No. 14 gauge or heavier, as required, and shall have a

properly sized fused discommect switch. Where the burmer motor electrical current requirements are for some other voltage than the control electrical current requirements, care must be taken to be sure the proper voltage is supplied to the controls, the burner motor, and any auxiliary equipment.

\section{FUEL LINE PIPING}

Refer to the separate Burner Installation and Service Nanual and any local or national code requirements which may apply to sizing and installing the fuel line piping and fuel tank.

\section{WARNINO BOILER MUST BE GAS-TIGHT}

For proper combustion efficiency and for safety, be sure the boiler is sealed gas-light. Correct any areas lhat are not gas-tight by wiping the outer surface of the asbestos rope with asbestus shorts dissolved in water-glass.

\section{BURNER ADJUSTMENT -}

Start the burner and adjust the air band so that a cleun yellow oil burner name with slightly smoky tips or a clean gas flame with slightly yellow tlps is established. Use combustion test instruments for final adjustment of the burner flame. A sinoke reading of a trace to $\mathrm{No} .1$ on the Shell Bacharach scale is recommended with 11-1/2 percent to $12-1 / 2$ percent $\mathrm{CO}_{2}$ for No. 2 fuel oil. $A \mathrm{CO}_{2}$ reading of 9 to 10 percent is recommended for natural gas. A CO test should be taken to assire that $\mathrm{CO}$ does not exceed .04 percent in the flue gases.

\section{BAROMETRIC DAMPER ADJUSTMENT}

When the burner is adjusted to the above combustion conditions, adjust the Barometric Draft Control to provide $-(1.02$ inches of draft overfire (below atmospheric pressures.

\section{WARNINO CLEAN THE NEW STEAM BOILER}

Now steam boilers must be cleaned properly previous to wi during the hirst few ditys of operation. follow the clouming recommendations listed on the (1perating lnstructions. Do not use chemical cleaners in this boller!

\section{ADDITIONAL INSTRUCTIONS}

Before leaving the job, make sure the unit checks elextrically and make sure the proper main burner name: is sceured. Be sure the room thermostat or operating control is atljusted to provide the des/red room temperature.

\section{BOILER SERVICE AND MAINTENANCE}

'he boiler Operiling Instructions contuin informatlon for the owner; review this information with the owner and/or the owner's representative und be sure he re ceives ull instructions. 
$A B C-573 \mathrm{~W}$ noth hi.to

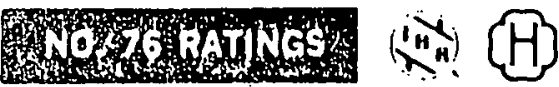

$$
\text { "gi" PllV Pirguage h lont }
$$

\begin{tabular}{|c|c|c|c|c|c|c|c|c|c|c|c|c|}
\hline \multirow{2}{*}{$\begin{array}{l}\text { Boiler } \\
\text { Unit } \\
\text { Nurnber }\end{array}$} & \multirow{2}{*}{$\begin{array}{l}\text { I.BR } \\
\text { Buiner } \\
\text { Copocily } \\
\text { liRlit oil } \\
\text { GPH }\end{array}$} & \multirow{2}{*}{$\begin{array}{l}\text { Gross } \\
\text { IBR } \\
\text { Oulput } \\
\text { Brught. }\end{array}$} & \multicolumn{3}{|c|}{ Not I.B R Ratingst } & \multirow{2}{*}{ 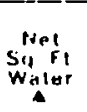 } & \multirow{2}{*}{$\begin{array}{l}\text { Broiler } \\
\text { H.P. }\end{array}$} & \multirow{2}{*}{$\begin{array}{l}\text { Net } \\
\text { fireliox } \\
\text { visluma } \\
\text { Cil. Fi }\end{array}$} & \multirow{2}{*}{$\begin{array}{c}\text { Slarch } \\
\text { Gias } \\
\text { volume } \\
\text { Cfis } \\
\text { (j) }\end{array}$} & \multirow{2}{*}{ 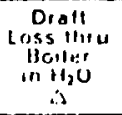 } & \multicolumn{2}{|c|}{ 1. B.R Chimnoy. b } \\
\hline & & & $\begin{array}{l}\text { Steam } \\
\text { Sq. ti. }\end{array}$ & $\begin{array}{l}\text { Sleam } \\
\text { BriJ/Hr. }\end{array}$ & $\begin{array}{l}\text { Woler } \\
\text { biujtir. }\end{array}$ & & & & & & $\begin{array}{c}\text { Size } \\
\text { Inchies }\end{array}$ & $\underset{\substack{H e a k h t t \\
\text { feet }}}{ }$ \\
\hline $\begin{array}{l}\text { BL-476-: } \\
\text { BL-576.: } \\
\text { BL-676: } \\
\text { BL-7 } \\
\text { BL. } \\
\text { BL. } 976 .: \\
\end{array}$ & $\begin{array}{l}2.35 \\
2.95 \\
3.60 \\
4.25 \\
4.90 \\
5.55\end{array}$ & $\begin{array}{l}264.000 \\
336.000 \\
408.000 \\
480.000 \\
552.000 \\
624.000\end{array}$ & $\begin{array}{l}825 \\
1.050 \\
1.275 \\
1.500 \\
1.725 \\
1.950\end{array}$ & $\begin{array}{l}198.000 \\
252.100 \\
306.100 \\
360.100 \\
414.100 \\
468.100\end{array}$ & $\begin{array}{l}239.508 \\
9354808 \\
417.400 \\
490.000 \\
542.600\end{array}$ & $\begin{array}{l}1.510 \\
1.950 \\
2.365 \\
2.785 \\
3.200 \\
3.615\end{array}$ & $\begin{array}{l}79 \\
10 \\
12 \\
14.3 \\
16.3 \\
16.5 \\
18.6\end{array}$ & $\begin{array}{r}425 \\
5.51 \\
681 \\
8.04 \\
9.37 \\
10.65\end{array}$ & $\begin{array}{l}147 \\
145 \\
226 \\
266 \\
307 \\
348\end{array}$ & $\begin{array}{l}.01 \\
.02 \\
.03 \\
.04 \\
.05 \\
.06\end{array}$ & $\begin{array}{r}8 \times 12 \\
8 \times \\
12 \times 12 \\
12 \times \\
12 \times 12 \\
12 \times 12 \\
12 \times 16\end{array}$ & $\begin{array}{l}15 \\
15 \\
17 \\
19 \\
21 \\
24\end{array}$ \\
\hline
\end{tabular}

NOTE: Add prelix "A" to designator for No. 76 Boiler with factory. (example: AB(.A76).

- Substitute ".S" lor steam. "W" for water For Tintermediale sections nedieto soctions with cover plates only, ado sutlin. (number cequiled)

- Burner inpul basod on iniaximum of 2,0001 . altitude-for other altitudes. Consult werlichain Customer Services Department.

DNo. 2 fuel oil-Coinmercial Standard Spec. CS12.48. Heat value of oil -

- Al combustion condition of $12 \frac{1 / 4}{1 \% \% \mathrm{CO}_{2}}$

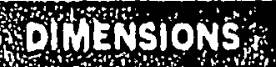

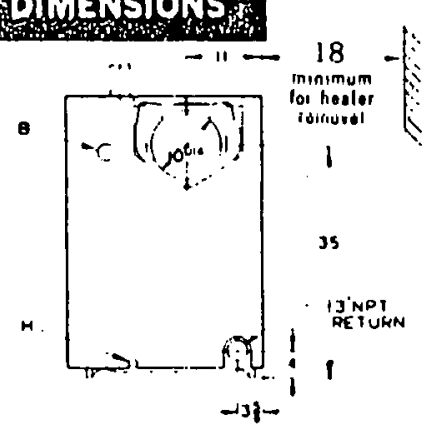

BACK

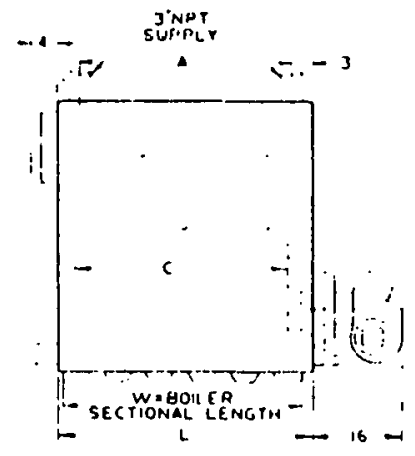

SIDE

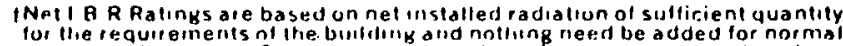

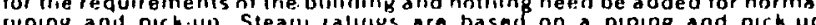
allowance of 1313 : water ratiligs on an allowance ol ipi 15 . An auditiona allowance should be made tar gravity hot water systenis or for unusual piping and pich.up lodis. Consult Custurner Services Departinent.

Adased un average water lemnerature of $170^{\circ} \mathrm{F}$. in radiators.

Stack gas volurie at outlet teinuerature.

ADraft over fire must the added to obtain dralt required at smoke collar. 1) When chiminey is lined with the larbest standard clay chimney lile. the equivalent area is considered the sanie as tha untrined chinmey area. NOIE: Water bollers available upon spectal request at 80 P.S.I. work ing pressure.

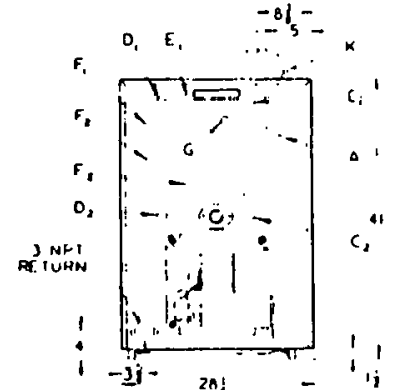

FRONT

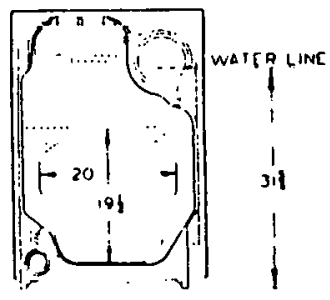

INTERMEDIATE

\begin{tabular}{|c|c|c|c|c|c|c|}
\hline \multirow{2}{*}{$\begin{array}{l}\text { Boiler } \\
\text { Nurnber }\end{array}$} & \multirow{2}{*}{$\begin{array}{l}\text { Supply Toppings } \\
\text { Number } \& \text { Sizo }\end{array}$} & \multirow{2}{*}{$\begin{array}{c}\text { Roturn Tappings } \\
\text { Number }\end{array}$} & \multicolumn{4}{|c|}{ Dimonslons (Inches) } \\
\hline & & & $\bar{A}$ & c & $\mathrm{L}$ & $w$ \\
\hline $\begin{array}{l}476 \\
576 \\
676 \\
776 \\
876 \\
976\end{array}$ & $\begin{array}{l}2=3 \\
2=3 \\
2=3 \\
2=3 \\
2=3 \\
2=3\end{array}$ & $\begin{array}{l}2=3 \\
2=3 \\
2=3 \\
2=3 \\
2=3 \\
2=3\end{array}$ & $\begin{array}{l}171 / 4 \\
231 / 4 \\
291 / 4 \\
351 / 4 \\
411 / 4 \\
471 / 4\end{array}$ & $\begin{array}{l}171 / 2 \\
231 / 2 \\
291 / 2 \\
351 / 2 \\
41 \% / 2 \\
471 / 2\end{array}$ & $\begin{array}{l}241 / 4 \\
301 / 4 \\
361 \% \\
421 / 4 \\
481 \% \\
541 / 4\end{array}$ & $\begin{array}{l}22 \\
28 \\
34 \\
40 \\
46 \\
52\end{array}$ \\
\hline
\end{tabular}

\begin{tabular}{|c|c|c|c|}
\hline \multicolumn{4}{|c|}{$\begin{array}{l}\text { DOMESTIC WATER HEATER CAPACITIES } \\
\text { TANKLLSS HEATCRS" }\end{array}$} \\
\hline $\begin{array}{l}\text { Healer } \\
\text { Mumber }\end{array}$ & $\begin{array}{c}\text { Intermiltent Draw GiPM } \\
100^{\circ} \text { Averoge } \\
\text { Temperaluie rise }\end{array}$ & $\begin{array}{c}\text { andinunus Diaw GPM } \\
100^{\circ} \\
\text { Tenneralure rise }\end{array}$ & $\begin{array}{c}\text { Inlet and } \\
\text { Outlet levpings }\end{array}$ \\
\hline 35529 & $1.5 \mathrm{GPi}$ & $4.0 \mathrm{cPm}$ & $\frac{\pi}{14}=$ \\
\hline
\end{tabular}

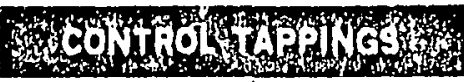

\begin{tabular}{|c|c|c|c|}
\hline Localion & Size & Stearn & Walel \\
\hline$A$ & $11 / 2 n$ & Salely Vatve and.or Shom Topping & High Limil Control \\
\hline B & $11 / \%$ & Salety valvo & Salely Relief Valvo \\
\hline$c_{1}$ & $I^{*}$ & - & $\begin{array}{l}\text { Combinalion High Limil and low } \\
\text { Limil Conlrol }\end{array}$ \\
\hline$C_{1} \& C_{2}$ & $1^{*}$ & Walet Level Conilols & Low-Woler Culon \\
\hline $0, \& 0_{2}^{\circ}$ & $1-$ & Water Level Controls & Low Watel Cuton \\
\hline$E_{1} \& E_{1}$ & h" & Goupe Glass & - \\
\hline $1,81_{1}^{\circ}$. & $1 / 1$ & Iiy Cosh Tepoings & - \\
\hline 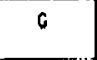 & 36 & $\begin{array}{l}\text { Pressure Limit Conlint Pressuie } O p \text {. } \\
\text { enating Conliol and Pressure Geuge }\end{array}$ & $\begin{array}{l}\text { Combinalion Pressure. } \\
\text { leenperalure Gauge }\end{array}$ \\
\hline H & $r^{*}$ & Diann & proin \\
\hline$x$ & $y^{2}$ & - & $\begin{array}{l}\text { Piping lo Curmpiession Tant or Auto. } \\
\text { malic All Vent }\end{array}$ \\
\hline
\end{tabular}

\section{Pror}

Insillated Flush lachet

lame Rotention Oil Burner

willi Casnuwin Cell and Primary

Gurrier Mountiris Plate with

Hefractory port on front
Observation

Section

tlue Collar

B.jlanreid Oraft Damper
low Vultage Tharmostat
flue Hrush

\section{WATER JOILER}

30 H.S.l ASME SAlety

Roliot Valve (uoriter

testell or his p.s

Huking bressure)

Cumbinalion

Pressurellem.

Bullitin Ai. Eluninator

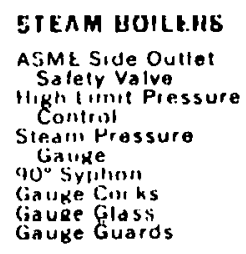

STENM BUILR.HB

ASME Side Oullet

High lint

Stealil Prossure

$90^{\circ}$ Syulum

Gave Glas
UNIJUNAL EQUUINAEN

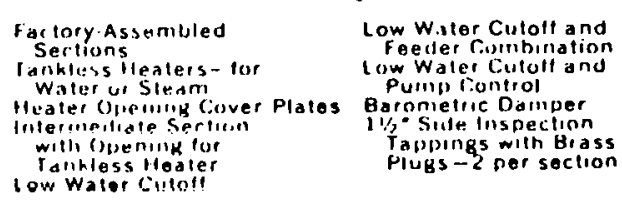




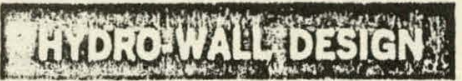

The No. 76 Builer has a water-backed combustion area with water circulating completely around the firehox. 'The crown aheet, sidewalls, and studs on the flue assages enlarge prime heating surface for maximum heat transfer.

In adolition to larger heating surface, Hydro-Wall section design also permits lower heipht, reduces heat less through the bottom of the boiler, eliminates the need for a refractury combust inn chamber, and permits installation on any flowr.

The cast iron sections are not faceground; the tough outer skin is retained to protect agaunst corrosion. 'J he 76 Builer is senled with asbestos rope.

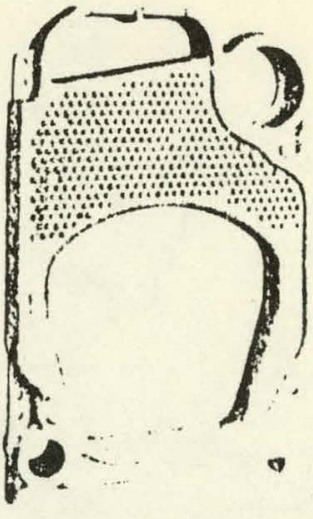

Potent No. $3,626,908$

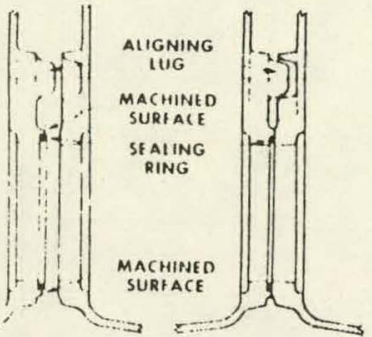

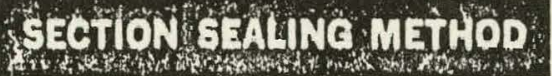

A flexible elastomer sealing ring is used in each port opening of the $\mathrm{No}$. 76 Boiler to assure a permanent, watertight seal hetween scctions. 'This sealing method, combined with the use of short draw rods to tie sections together, also permits faster section assembly.

As nhown in the illustrations, the machined surface of the port opening controls the compression ratio of the sealing ring for a watertight seal. The aligning lugs assure prover section alignment during assembly and positive locking of the sections.
The No. 76 can be cleaned from the tou of the briler hy removing the top jacket panels. The steel cleanout plates are sealed with ashestos rope and are easily removed to expose the section heating surfaces for cleaning and inspection.

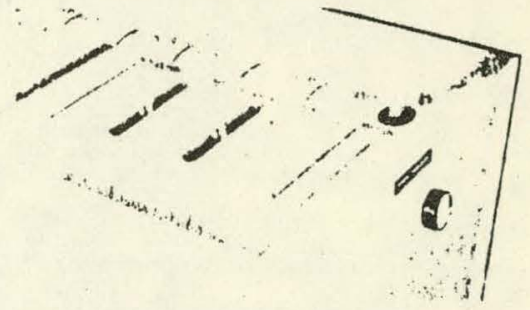

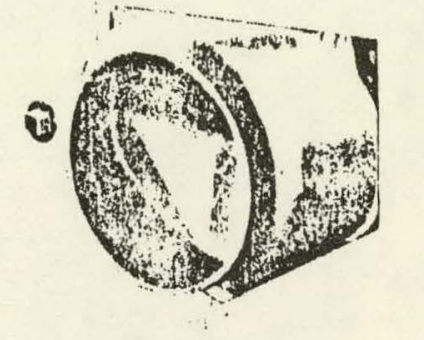

A horizontal flueway. cast inte, No, 76 sec tims, serves as a flue gas callectrir. This fenture folminates the need for a separate sheet metal col. lector liosid.

The standard equipment cast iron flue collar permits a straight-through connection to the chimney.

\section{MULI LLE TANKLESS WATER HEATEZS}

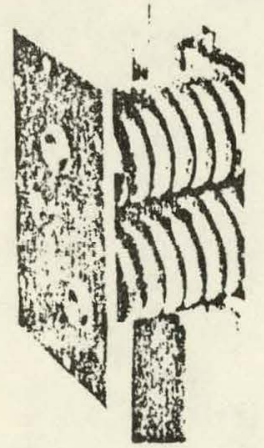

Tankless heaters for the No. 76 Boiler are installed in the left side of the boiler in intermediate sections with heater openings.

Multiple heaters offer these advan. tages: (1) illoteaned domestic hot water. (2) hel water at different temupelatures, and lis one heater for sonow melting application.

\section{SECTION ASSEMBLY FOR TANKLESS HEATERS}

\begin{tabular}{|c|c|c|}
\hline $\begin{array}{l}\text { Builer } \\
\text { No. }\end{array}$ & $\begin{array}{c}\text { Maximum No. } \\
\text { of Heaters }\end{array}$ & $\begin{array}{l}\text { Section Assernbly } \\
\text { All heaters must be on left side of hoiler }\end{array}$ \\
\hline 476 & 1 & $F \| 11 B$ \\
\hline 576 & 2 & 1.111 .118 \\
\hline $6 ? 6$ & 2 & \& $11.111 .1 \mathrm{~B}$ \\
\hline 776 & 3 & $1111 \cdot 1.11 B$ \\
\hline 876 & 3 & 1.1111111118 \\
\hline 976 & 4 & F. $\left\|1.11 .1^{-}-1\right\| B$ \\
\hline
\end{tabular}

F Front Section, B Back Section, I Intermediate Section 11 Intermediate Section with Tankless Heater Opening

TANKLESS HEATER LOCATIONS - II TI sechIOns are udered. it is important to pusition them in the tovier seclion assembly exactly as shown by the 11 symbols in the tat)le atove to match lacket knockouts and assure proper operation. An asterisk ( $)$ in the latule for the 776 and 976 indicates a TI section can ve installed in this position bui the wistaller must rut the lacket opening since a knochout is not provided

\section{RECOMMENDE PRPIC CONNECTIONS}
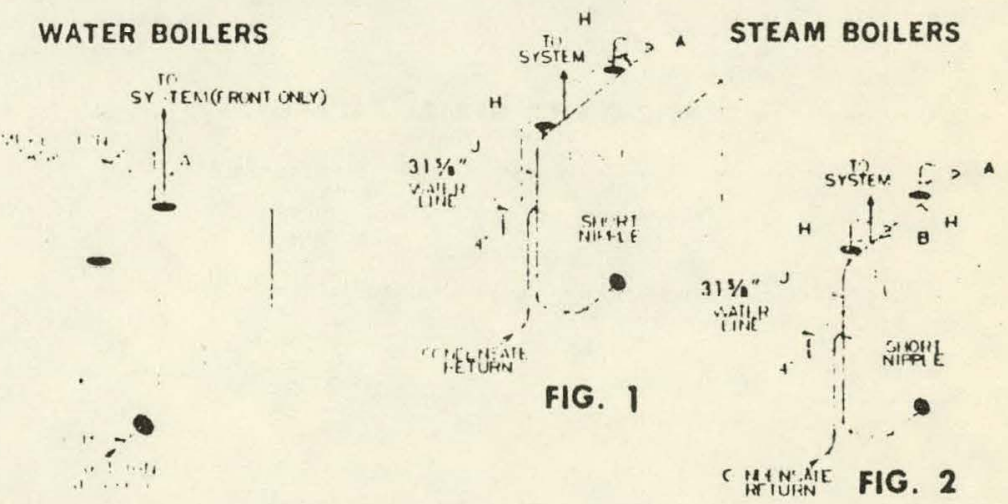

NOTE: Supply and return sizes for water boilers refer to minimum size of pipe conntected to boiler for 20 or higher temperature drop between suip)ly alld iefurn.

\begin{tabular}{|c|c|c|c|c|c|c|c|c|}
\hline \multirow{2}{*}{$\begin{array}{c}\text { Water } \\
\text { Boiler } \\
\text { Size }\end{array}$} & \multicolumn{2}{|c|}{ Pipe Size" } & \multirow{2}{*}{$\begin{array}{l}\text { Fig. } \\
\text { No. }\end{array}$} & \multirow{2}{*}{$\begin{array}{c}\text { Steam } \\
\text { Boiler } \\
\text { Size }\end{array}$} & \multicolumn{2}{|c|}{$\begin{array}{c}\text { Riser } \\
\text { Pipe Size }\end{array}$} & \multirow{2}{*}{$\begin{array}{c}\text { Header } \\
\mathrm{H}\end{array}$} & \multirow{2}{*}{ Equalizer } \\
\hline & $\underset{\text { (Sup. }}{A}$ & $\begin{array}{c}\text { B } \\
\text { (Re. }\end{array}$ & & & & $\begin{array}{c}\text { Size } \\
\text { B }\end{array}$ & & \\
\hline 475 and & $p(y)$ & turii) & 1 & $\begin{array}{c}476 \text { and } \\
576\end{array}$ & $3^{\prime \prime}$ & - & $3^{\prime \prime}$ & $1 \frac{1 / 2 "}{1}$ \\
\hline $\begin{array}{c}576 \\
676 \text { and }\end{array}$ & $2^{\prime \prime}$ & $2^{\prime \prime}$ & 2 & $\begin{array}{c}676 \text { and } \\
776\end{array}$ & $21 / 2^{n}$ & $21 / 2 "$ & $4^{\prime \prime}$ & $11 / 2^{\prime \prime}$ \\
\hline $\begin{array}{c}776 \\
876 \text { and }\end{array}$ & $21 / 2 n$ & $21 / 2$ & 2 & $\begin{array}{c}876 \text { and } \\
976\end{array}$ & $3^{\prime \prime}$ & $3 "$ & $4^{\prime \prime}$ & $2^{\prime \prime}$ \\
\hline 976 & $3^{\prime \prime}$ & $3^{\prime \prime}$ & & minimur & from & vatet & ine to heac & der. \\
\hline
\end{tabular}

- For minimum of 10 pipe diallietets itoni ivoller. 


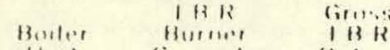

limi (ind)

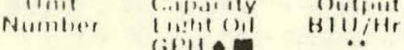

Si. $1,+1,11$

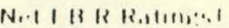

\begin{tabular}{|c|c|}
\hline $\begin{array}{l}35 \\
4 \\
41,\end{array}$ & $26,4,0(1)$ \\
\hline '4', & $336,(1)(1)$ \\
\hline 3.t, & $\begin{array}{l}408,(1) \\
48000\end{array}$ \\
\hline 点 & $\begin{array}{l}480 .(1) \\
55 ?(1)\end{array}$ \\
\hline 56 & 6.400 \\
\hline
\end{tabular}

sili,ili Hili,

$+-4760^{\circ} \quad \because 35, \quad 26,0(0)(1)$

-576.0
+-676.0

$+.7760^{\circ}$

$\begin{array}{ll}+-8760^{\circ} & 490 \\ +-9760^{\circ} & 5,56\end{array}$

f.' 000

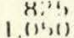

$1, ., 14$

i,ili

1. 950

198 (1)(3)

24. I(11)

3(1), 11)

(1, (). $1(1)$

414.10

468.100

$\because,{ }^{2},(1)$

("', '(II)

(1) $(1,0)(1)$

4 (i) () ()

5.9., (1)

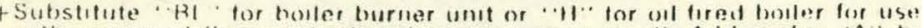

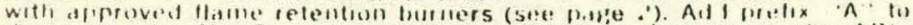
designator for No. 76 with tactory assembled sertions (exalin)te. ABL. 476 or All 476 .

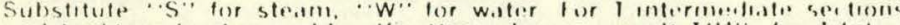

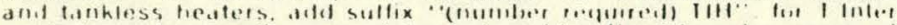

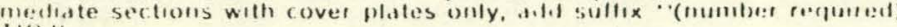
III.

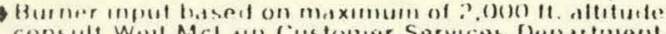

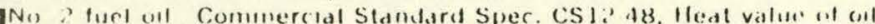
(4) ()() 8 \&II/G.

\section{DimeNSIONS}

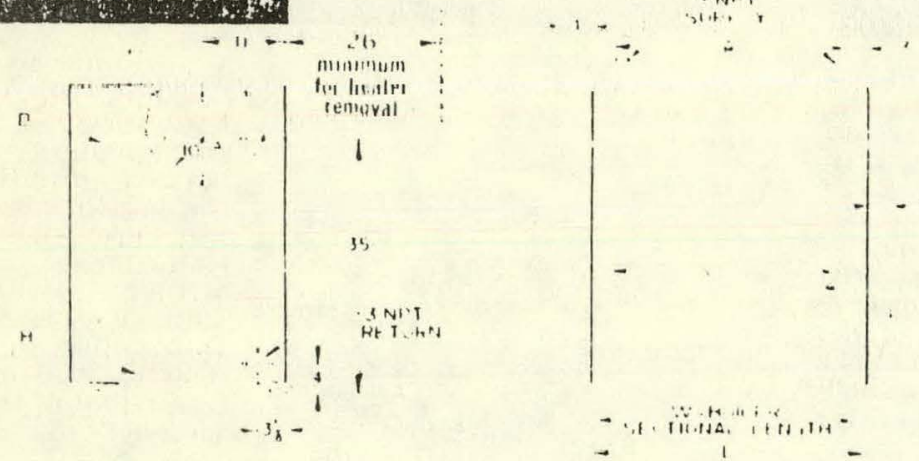

BACK

SIDE

winter Number \& Size
$476 \quad \because \cdots 3$

$476 \quad \because 36$

6,6

77

$7>6$
976

?"

$\therefore$ ?"
Return lappentise Number \&s size

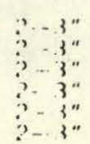

Dimensions (Inches)

\begin{tabular}{|c|c|c|c|}
\hline A & C & 1 & v \\
\hline 11 & 11 & $\therefore A^{\prime}-1$ & \\
\hline 3 & $\because 31$ & 3()$: 4$ & \\
\hline $\begin{array}{l}34 \\
35\end{array}$ & 351\% & $\begin{array}{l}3619 \\
4111\end{array}$ & \\
\hline 4114 & 411 & $\Delta Y^{\prime}:$ & \\
\hline & & & \\
\hline
\end{tabular}

DOMESTIC WATER HEATER CAPACITIES IANKLESS HEAIEKS

\begin{tabular}{|c|c|c|c|}
\hline $\begin{array}{l}\text { Hextes } \\
\text { Humber }\end{array}$ & 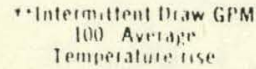 & 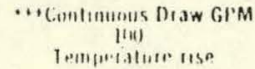 & $\begin{array}{c}\text { lutel and } \\
\text { Outipt lagents }\end{array}$ \\
\hline 23 & $4.5 \mathrm{GPM}$ & $4.0 \mathrm{GPM}$ & $\because "$ \\
\hline
\end{tabular}

Weil Mi. I in Ratiens

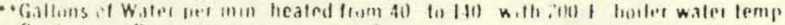

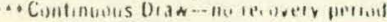

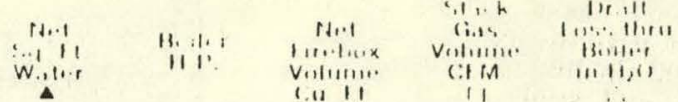

I B R I Tum

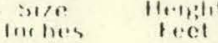

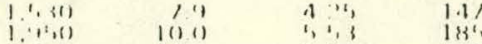

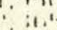

$\therefore 185$

$3, i, 1: 3$

Ii,

1, $1: 1$

is (1)

43

1,11
$13 i^{\prime}$,

?hi,

3(1)

318 (itim in 11,1,

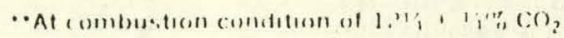

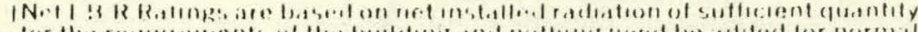

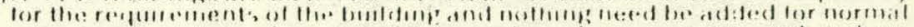

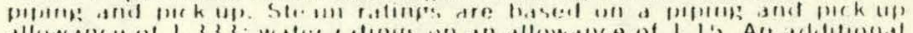

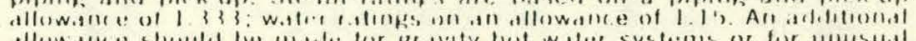

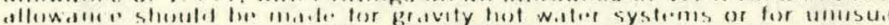

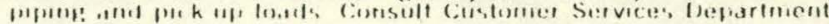

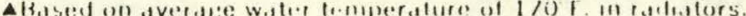

Stack fas volutite al outlet leamperatute.

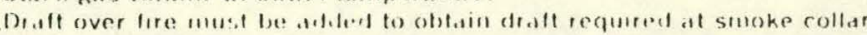

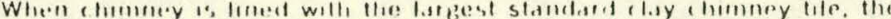

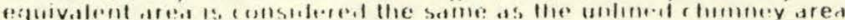

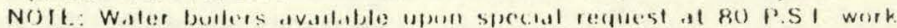
ung pressure.

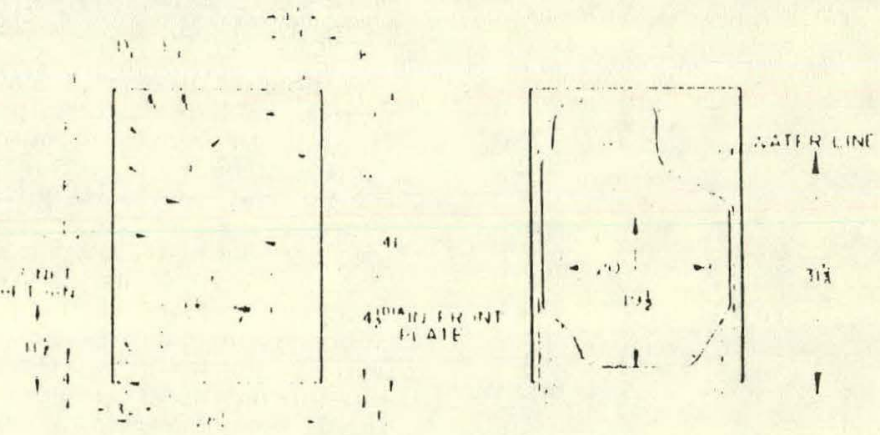

FRONT

INTERMEDIATE

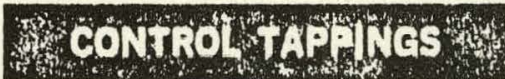

\begin{tabular}{|c|c|c|c|}
\hline Lutation & Size & Steam & Water \\
\hline$\wedge$ & $11:$ & Sately Volve and ot Skim Iappunp? & Hogh L Laul Cortral \\
\hline n & $I^{\prime}: \cdot$ & $\therefore$ sitit, Vallu & S.tely Reluet Value \\
\hline$c_{1}$ & $1^{\prime \prime}$ & $m$ & 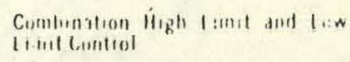 \\
\hline$c_{1} x c_{2}$ & $1 *$ & Wat. l l...l Cutitols & I IN Wat.r Cutuff \\
\hline$v_{1} \& v_{2}$ & $1^{\prime \prime}$ & Vittel livel Cimltois & Inw Water Cufull \\
\hline$t, \& 1$ & . & batuen tilatis & - \\
\hline 1,81, & $\because$ & 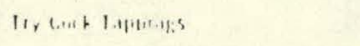 & - \\
\hline$c_{i}$ & , & 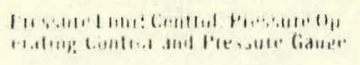 & 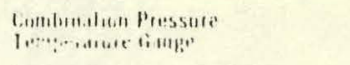 \\
\hline$H$ & $\because$ & U1, & Dha! \\
\hline r. & $\therefore$ & - & 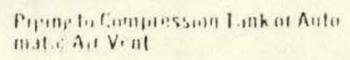 \\
\hline
\end{tabular}

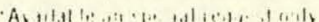

\section{STLDARDEOUTHEM}

\section{ALL BOILERS}

Insuldetent flush l.t her Busfer Montulas: Plole with Roltatioty

Oteservestiun fort on t rom Secturl

flue collar

Batlancest Dratt Domber

Flue Brinst

For BL-76 Only

hime: Relention (1) Bumbe

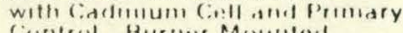

uw Voltape Thermostit
ALL WATER BOILERS

(1) 1 S I A SMI sufety kither valves (lmale? testert tor sit) PS. worthos? pres.

P'ressurellent

Hetsture Ci.tust.

Built in Alt linimaster

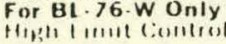

\section{ALL STEAM BOILERS}

ASMI S. H. Hulle.

Solte Iy Valve

St?alli)

(i) Sylilut

(i.311) lituk k

(i.111):0 (1,1,12.

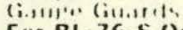

For BL-76.5 Only

Heph I In..1
OPTIONAL EQUIPMENT

1." lory Awermbled

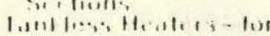

Wister on Ste.ent

Mredter Omentin: Lover Flates

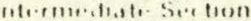

with (1)

low whter coutstl
Low Woter Contofl and

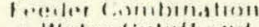
How Wale! Coutor

Baremetru: Detmper

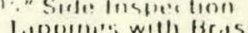

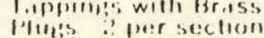


ATTENTION HEATING CONTRACTOR: This warranty is for the building owner and should t? given to him or placed in sight near the boiler.

a. ?

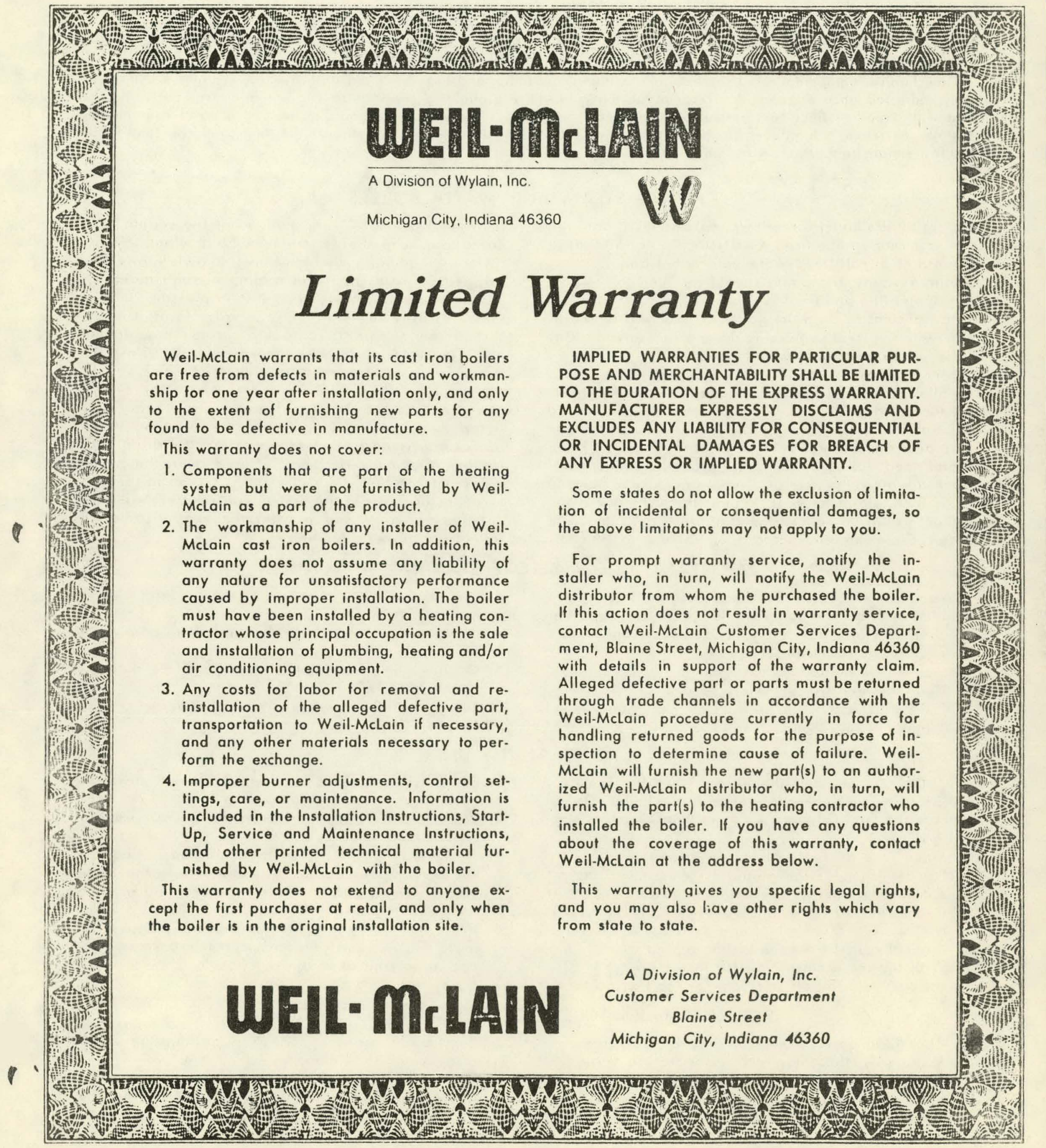




\section{PLEASE HANG THIS CARD NEAR THE BOILER OPERATING INSTRUCTIONS for WEIL-MCLAIN OIL, GAS OR GAS/OIL BOILER}

Mr. Boiler Owner:

Below, the procedure is outlined for starting your Weil-McLain Boiler including instructions for the care of your heating system.

All mechanical equipment needs occasional attention. Your boiler and burner should be inspected, cleaned and if necessary, adjusted once a year. We recommend that you utilize a qualified serviceman who has been trained for the job and will have the necessary instruments to check your boiler. This will assure you that the operation of your heating system will remain highly efficient. Your Weil-McLain boiler will give you a lifetime of heating comfort, if you follow the few simple suggestions listed on this card.

\section{FILLING STEAM AND WATER BOILERS}

Do not fill the boiler (except for leakage tests) until the builer is ready to be tired. CAUTION: Do not add large quantities of cold feed water tn any hot boiler!

Steam Systems: The builer should be tilled to the normal water line and fired for about 15 minutes at a low rate sufficient to keep the boiler at steaming temperalure with the steam vented to drive off dissolved guses (also see Skimming Steam Boilers).

Water Systems: The boiler and the entire system should be filled to about 12 pounds per square inch and heat. ed to approximately $210 \mathrm{~F}$ for about 15 minutes to drive off dissolved gases. Before filling the system, make sure all the system air vents are closed. Open the hand water feed valve and beginning on the lower floor, open the air vents (one at a time) until water starts to flow; then, close the vent. Repeat this throughout the building until all heat distributing units are filled with water. Close the hand water feed valve when the cor- rect boiler pressure is reuched. After the system is in operation, keep the system filled with water by nccasionally opening the air vents allowiny uny entrapped air to escape and adding enough make up water to mainfain the correct system pressure. If your system is provided with a purge valve located in the system return piping, connect a garden hose to the drain valve located above the purge valve. Close the purge valve and open the hand water feed valve and allow the system to purge all air. Where the system has more than one circuit, purge each circuit separately by opening each balancing valve one at a time. When the system is purged of all air, close the drain cock located above the purge valve and open the purge valve. Fill the boiler and the entire system to the correct pressure. Air in the system can interfere with circulation of water and prevent the heat distributing units from properly heating.

\section{IMPORTANT: CHECK BEFORE STARTING THE UNIT}

Make sure the boiler heating surfaces have been cleaned and that the boiler is filled to the correct water level or pressure. It is recommended that your burner serviceman be called to service your burner and check the following points:

1. Clean the fuel strainer, where used.

2. If there is a filter in the fuel oil line, clean it and change the cartridge.
3. Clean all lint and dust out of the fan and blower housing.

4. Check the ignition electrodes and clean or replace the nozzle where used.

5. Oil the burner motor with detergent free automobile engine oil.

6. Rotate the blower wheel by hand to make certain if turns free.

\section{TO START THE UNIT}

CAUTION: Do not start the burner when the firebox is hol or when fuel vapor is present in the boiler. Do not operate the Fuel Oil Pump for more than 2 minutes without fuel nil

1. Make sure all fuel valves are open.

2. For one-pipe fuel oil piping systems, bleed the air out of the piping by opening the unused intake port on the Fuel Oil Pump and waiting for the oil to flow. For iwo-pipe fuel oil piping systems, air is auto. matically bled from the system piping.

3. With the main electric switch in the burner electri. cal circuit in the off position, set the thermostat or operating curiliul at a point which will start the burner.

4. Push the safety reset lever on the burner primuiy control and release.

5. Turn the muin electric switch to the on position. If the burner does not start instantly, furn the main electric switch back to the off position and refer to the following section titled If Burner Fails To Start.

6. If the burner starts to operate normally, leave the main electric switch on and reset the thermostat or operating control to desired position.

\section{IF BURNER FAILS TO START, CHECK THE FOLLOWING}

1. Check for loose connections and blown fuses.

2. Make sure the thermostat temperature setting is above the room temperature.

3. Be sure all fuel valves are open.

4. Be sure there is sufficient fuel oil in the tank to supply the burner.
5. Reset burner primary control by pushing the safefy reset lever and releasing.

6. Push reset button on the burner motor.

7. If the burner does not start after observing the above checks, call your serviceman. 


\section{SKIMMING STEAM BOILERS}

All new boilers and steam and water piping contain oil, grease, chips, and other foreign matter. It is essen. tial to clean new heating systems to remove these materials in order to avoid overheating of boiler metal, foaming and priming, and high maintenance costs on strainers, traps, and vents. The boiler installer should use the following procedure to clean oil, grease, and other impurities from the new boiler:

1. Close the valves in the building steam supply $\operatorname{main}(\mathrm{s})$.

2. Provide a $1 \frac{1 / 4}{\prime \prime}$ to $2 \frac{1}{2}$ " skim line, with valve, from the boiler skim tapping and run this line to a convenient floor drain.

3. Fire the boiler at a low rate sufficient to keep the hoiler at steaming temperature allowing the steam, along with entrained water and impurities, to discharge through the skim piping to the drain.

4. Feed the water to the boiler as required to maintain proper water level in the gauge glass. It may be necessary to cycle the burner to prevent a rise in steam pressure above several pounds.

5. Continue the boiling and skimming process for at least two hours or until the water leaving the skim line is clear of all grease, oil and impurities. On unusual jobs, the skimming procedure may require repeating one or more times.

CAUTION - THE USE OF CHEMICAL CLEANERS IS NOT RECOMMENDED!

6. Drain boiler and, while boiler is warm but NOT HOT, remove safety valve and insert a hose nozzle into the opening. Flush all interior surfaces of the boiler with water under full pressure until all traces of dirt and impurities are removed and the drain water runs clear.

7. Replace safety valve; close drain cock; fill with fresh water to the water-line. Start burner and steam for 15 minutes to remove all dissolved gases; stop burner.

8. Drain boiler sufficiently to remove skim piping; plug skim tapping; refill boiler to waterline.

9. To prevent the return of impurities to the boiler from new or old piping systems, waste all condensate for several days or until no impurities are contained in the condensate. NOTE - IT IS IMPERATIVE THAT FEEDWATER BE SUPPLIED TO MAINTAIN THE CORRECT WATER LEVEL AND THAT A LOW WATER CUT. OFF IS OPERATIVE!

\section{BOILER SERVICE AND MAINTENANCE}

Leaks in the boiler and piping system must be repaired at once. The use of makeup water in large quantities is undesirable and may damage the boiler after an extended period of time. If serious leaks occur, stop the burner and gradually reduce boiler pressure or temperature. Do not attempt to make repairs while a steam boiler has pressure or hot water boiler tempera. fures are above $130^{\circ} \mathrm{F}$. Pefroleum based products should not be used for cleaning or sealing this boiler. Foaming or priming may occur in a steam boiler and cause large quantities of water to pass out into the steam main(s). It can be observed by violent fluctuations of water level, in the gauge glass. This trouble may be caused by dirt, oil, or precipitates in the boiler water, too high a boiler water level, a high overload on the boiler (ie, the sudden release of boiler steam pressure into the mains by action of fast operating vulves), or the addition of ton murh boiler water treatment. With serious foaming or priming, stop the burner and decrease boiler load. Then alternately blowdown and slowly feed fresh water several times. If trouble persists, it may be necessary to skim the boiler one or more additional times.

Any problem in regard to large amounts of makeup water, extreme foaming or priming, scale in the boiler, or internal corrosion or pitting, should be referred to a company specializing in boiler water chemistry. DO NOT try "homemade cures" or boiler "patent medicines" on the market under various trade names, as serious damage to the boiler, personnel, and property may result.

Frequently check the boiler water level in the gauge glass of steam boilers, and check the boiler operating pressure of steam or water boilers. Test the low water cutoff by opening its blowdown valve to remove dirt, rust, and sediment, and observe that burner stops as the water levei approaches the bottom of the water gauge glass (gauge glass on steam boilers only).

Refer to Water Boiler Controls or Steam Boiler Controls for specific service requirements.

Refer to Cleaning Boiler Heating Surfaces for periodic cleaning of your boiler.

On steam boilers, open boiler blowdown valve and flush till clear while under sleam pressure. On water boilers, open boiler drain cock to remove impurities that have settled to the bottom of the boiler. Refill as required to the correct water line for steam boilers or the correct pressure for water boilers.

\section{SHUT DOWN OF BOILER}

DO NOT DRAIN BOILER during periods of shutdown unless heating system is exposed to freezing temperafures.

1. Turn off the main electric switch.

2. Close all fuel valves in the fuel lines.

3. Cover the burner to profect it from dust and damp. ness.

4. Open the boiler drain cock to remove impurities that may have settled to the bottom of the boiler; it may be necessary to drain one or two gallons of water until all traces of sediment are gone. Refill the boiler to the proper water level or pressure (see Filling Heating System). Boiler woter does not have to be crystal clear for proper operation, but should

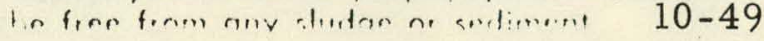

5. During severe winter weather have heating system operation checked periodically or thoroughly drain your heating system.

Where it becomes necessary to drain the heating system for an extended period, follow items 1, 2 and 3 above. Then, clean all carbon, rust, and other deposits from the fire-side of the boiler heating surfaces in order to protecl the boiler from the corrosive action of combustion deposits (see Cleaning Boiler Heating Surfaces). Apply a thin coating of nil or grease if the boiler is to remain ouf of service for extended periods of time. If the water side of the boiler must be cleaned or inspected, open the blowdown valve and drain the boiler.

Remove plugs from the boiler and open the drain cock. Hose the inside of the boiler with high pressure water to remour slintra.. and sediment flush nquin. Dry insides of 
boiler thoroughly, or refill with fresh water and heat to re. lease dissolved gases (see Filling Steam and Water Boil. ers). Repeated draining and filling of the boiler and/or the heating system can lead to the same consequences as add- ing too much makeup water - this is mainly true where the makeup water is "hard" and the same precautions must be used as indicated in the third paragraph of this section.

\section{CLEANING BOILER HEATING (fire-side) SURFACES}

At the end of every heating season, it is advisable to clean the flueways in the boiler. Soot is an effective insulator and prevents the hot gases from heating the boiler water as efficiently as possible. The frequency of cleaning will depend upon the fuel used, the burner adjustments, boiler temperature, draft conditions, and other job factors.

Normally the boiler flueways will be cleaned as described below:

1. Remove the flueway opening cover(s).

2. Cover the burner and controls.

3. Insert the wire flue brush supplied with the boiler into the flueways and thoroughly brush the flueways at ull ungles.

4. Remove any soot or scale from the horizontal flueways, the boiler firebox and the base of the chimney using a vacuum cleaner or brush. Do not brush or vacuum the firebox or combustion chamber area of those boilers using liberous combustion chamber materiall
5. Replace the flueway opening cover(s) making sure the original gas-tight seal is maintained.

Chemical additives which can be procurred from most heating supply houses or from your fuel oil supplier can be effectively used to clean the boiler flueways; certain types of chemical additives can be added to the fuel oil while other types can be placed on the floor of the firebox. The use of stick type thermal bombs is not recommended. Consult your heating contractor or fuel oil supplier before using any chemicals in the boiler.

The boiler flueways can also be cleaned by heating the boiler to $180 \mathrm{~F}$.; then, removing the flueway opening cover(s). Use a garden sprayer or connect a spray nozzle to a gaiden huse and spray the sooted flueways with a fine mist of water; the soot will become loosened and fall to the floor of the fircbox and uruund the hori. zontal flueways. Remove the loosened soot from the horizontal fueways and from the floor of the firebox using a vucuum cleaner or brush except those boilers using fiberous combustion chamber material.

\section{BURNER ADJUSTMENT}

Refer to the burner manual for proper burner adjust ment; your serviceman should properly use combustion test instruments for efficient operation. The flame must not strike (impinge) against any heating surfaces within the boiler firebox.

\section{WATER BOILER CONTROLS}

\section{CIRCULATOR CARE:}

Never operate the circulator without water.

A. Follow lubricating instructions on circulators that are provided with oil cups or oil holes.

B. Follow venting instructions on circulators with water lubricated bearings which require no oil.

BOILER PRESSURE: The initial fill pressure of a hot water system is generally to 12 pounds per square inch. When the system is heated to the limit control setting, the pressure may range up to 30 pounds per square inch. Normal system pressure will fluctuate between the fill pressure, when the system is cold; and up to 20 to 28 pounds per square inch when the system is hot.

BOILER WATER TEMPERATURE: Modern hot water heating systems with "closed" type expansion tanks may operate at water temperatures up to $250 \mathrm{~F}$. Set the high limit control at $220 \mathrm{~F}$; during severe weather you may find this temperature setting needs to be raised or lowered, depending upon the characteristics of your heating sys. tem. Those heating systems furnishing hot faucet water must have a low limit control set at a temperature which will furnish an adequate supply of domestic hot water; set low limit onntrol $20 \mathrm{~F}$ below high limil setting and use a 15 ' $\mathrm{F}$ differential setting.

PRESSURE-TEMPERATURE-ALTITUDE GAUGE: This gauge indicates the boiler pressure in pounds.per-square-inch and in feet of water column (altitude) above the boiler by the moveable hand The fixed hand may be changed to indicate the proper position for the moveable hand on manually filled hot water heating systenis. For those systems with automatic fill valves, the fixed hand is usually left at the zero setting. The third hand indicates the boiler water temperature in degrees fahrenheit.

FLOAT TYPE AIR VENT: If your system is provided with a Float Type Air Vent(s) which automatically expells air from the heating system, when the system is filled with water, loosen cap (A) slowly sn that particles of dirl ur scale are not deposited on the valve seat by the escaping air. Should dirt or scale lodge on the valve seat causing it to leak, remove cup and push the valve core (B) in by hand to permit water to flush the valve seat clean. Release the valve core quick. ly and replace cap. For normal operation and venting, unscrew the cap at least two turns.

WATER RELIEF VALVE: Check the relief valve at least once a year by pulling the handle and allowing a small quantity of water to flow. Be sure the relief valve reseats properly and is entirely free from seepage. If the relief valve sticks or

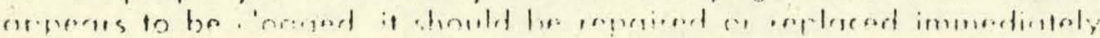

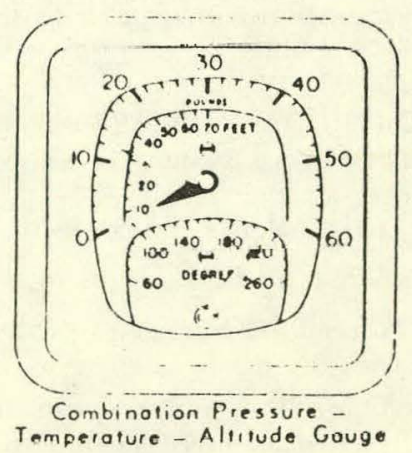

$10-50$ 
COMPRESSION TANK: Compression tank(s) are employed with hot water heat. ing systems to accept the increased water volume which results from heating the system water. The compression tank on a closed hot water heating system should provide adequate pressurization under all system operating conditions. Frequent opening of the pressure relief valve can be the result of an undersized compression tank because provision for the necessary expanded water volume has not been provided. Compression tanks may be of the open, closed or closed diaphragm type.

Open Type Expansion Tank: Open type expansion tanks are located above the highest heat distributing unit in the system usually in a closet or attic space and equipped with a gauge glass and an overflow pipe to a drain. The open type expansion tank and drain piping should not be located in any area where freezing temperatures could occur.

Closed Type Expansion Tank: Closed type expansion tanks are welded gastight and are usually located just above the boiler but may be located at any point in the heating system. In order to utilize the built-in air elimination system on certain boilers, the closed type compression tank must be piped to the air elimination tapping on the boiler. When the system is initially filled with water, a cushion of air is trapped within the tank and this air cushion is compressed to provide the initial fill pressure. When the system is heated, the expansion of the system water further compresses the air cushion and provides the additional space required for the additional water volume. A rapid increase in boiler pressure with frequent opening of the pressure relief valve during warm-up of the boiler and heating system usually indicates a "waterloqged" compression tank. Your serviceman should be called to correct this condition by partically draining the compression tank to again establish an air cushion.

Closed Diaphragm Type Compression Tank: Closed diaphragm type compression tanks are welded gas-tight and a rubber diaphragm is employed to separate the air cushion from the system water. The closed diaphragm type expansion tank may be located at any point within the heating system but is usually located as close to the boiler as possible. Where a closed diaphragm type expansion tank is employed, an automatic air-eliminating device should be installed in the air elimination tapping(s) on certain boilers to provide system air control. Before the initial fill of the heating system, the closed diaphragm type compression tank should be charged with air (by means of a tire pump) to a pressure equal to the initial fill pressure; the lank pressure may be checked by means of an air pressure gauge. As the system is filled, water will not enter the tank until the system pressure exceeds the lank charge. When the system is heated, the expansion of the system water causes the diaphragm to flex and further compress the air cushion and additional space is provided for the additional water volume. Since the system water is separated from the air cushion by means of the diaphragm, absorption of the air cushion by the system water is eliminated.

\section{STEAM BOILER CONTROLS}

BOILER PRESSURE. Steam boiler pressures may range up to 15 psig maximum, but in normal service usually will not exceed 5 psig and may even operate under vacuum conditions at cerrain limes.

The compound gauge used for steam boilers indicates steam pressure in pounds per square inch (psig) and boiler vacuum in inches of mercury (hg). CLEANING LOW WATER CUT-OFF. Accumulated sediment in the low water cutoff should be flushed out through a blow-off valve provided for this purpose at least once each month of heating system operation.

CLEANING THE GAUGE GLASS. This may be done by closing the lower gauge glass cock and carefully opening the petcock below the glass to blow water and sediment out of the gauge glass by sleam pressure. Then slowly open the lower gauge glass cock, allowing a small amount of water to flush out through the open petcock. Close petcock and fully open the lower gauge cock. The water level should immediately rise to its proper level. If gauge glass breaks, close off both gauge cocks and loosen glass retaining nuts to remove gauge glass. Replace broken gauge glass with new gauge glass made of heavy walled pyrex. DO NOT USE THIN GLASS TUBING!

CHECKING THE SAFETY VALVE. The safefy valve should open at 15 psig to prevent excessive boiler pressure. Manually open the safety valve once each year by pulling the valve lever or handle and allowing a small amount of steam to escape. This will help to assure proper operation of the safely valve if boiler pressures reach 15 psig. Be sure that the valve reseats properly and does not leak steam. If the safety valve sticks or appears to be clogged it should be repaired or replaced immediately by your serviceman.
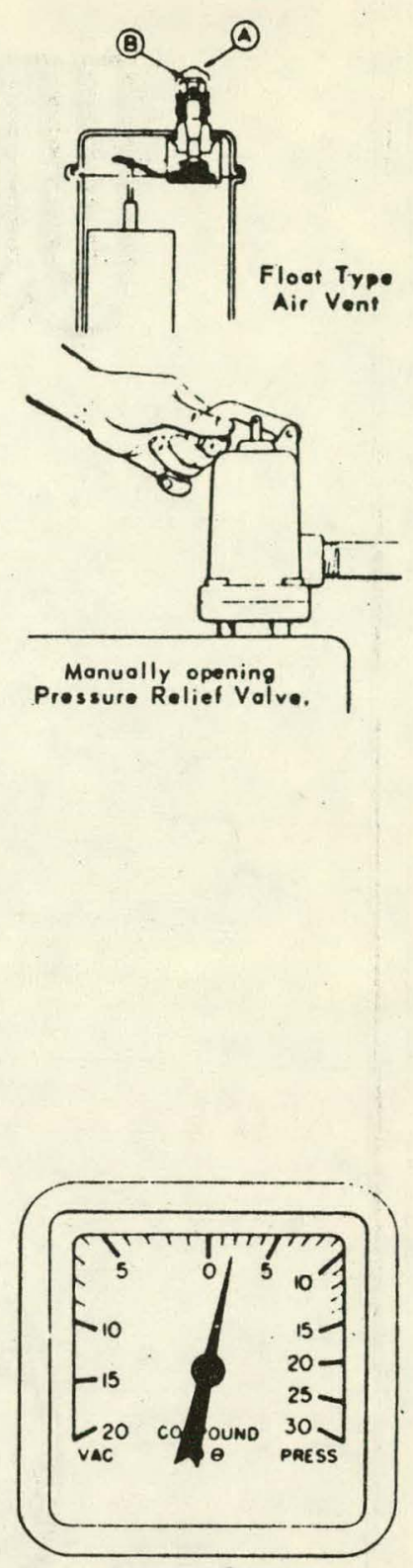

Pressure Gauge.

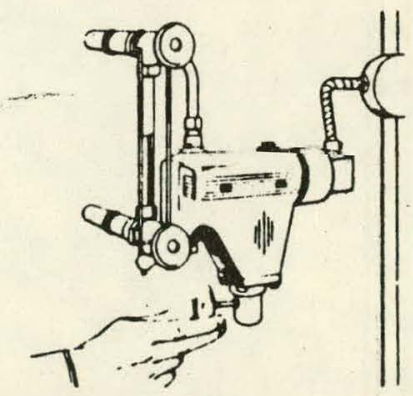

Flushing Low Woter Cut-off.

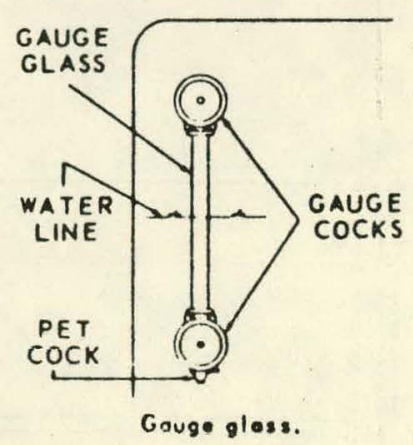




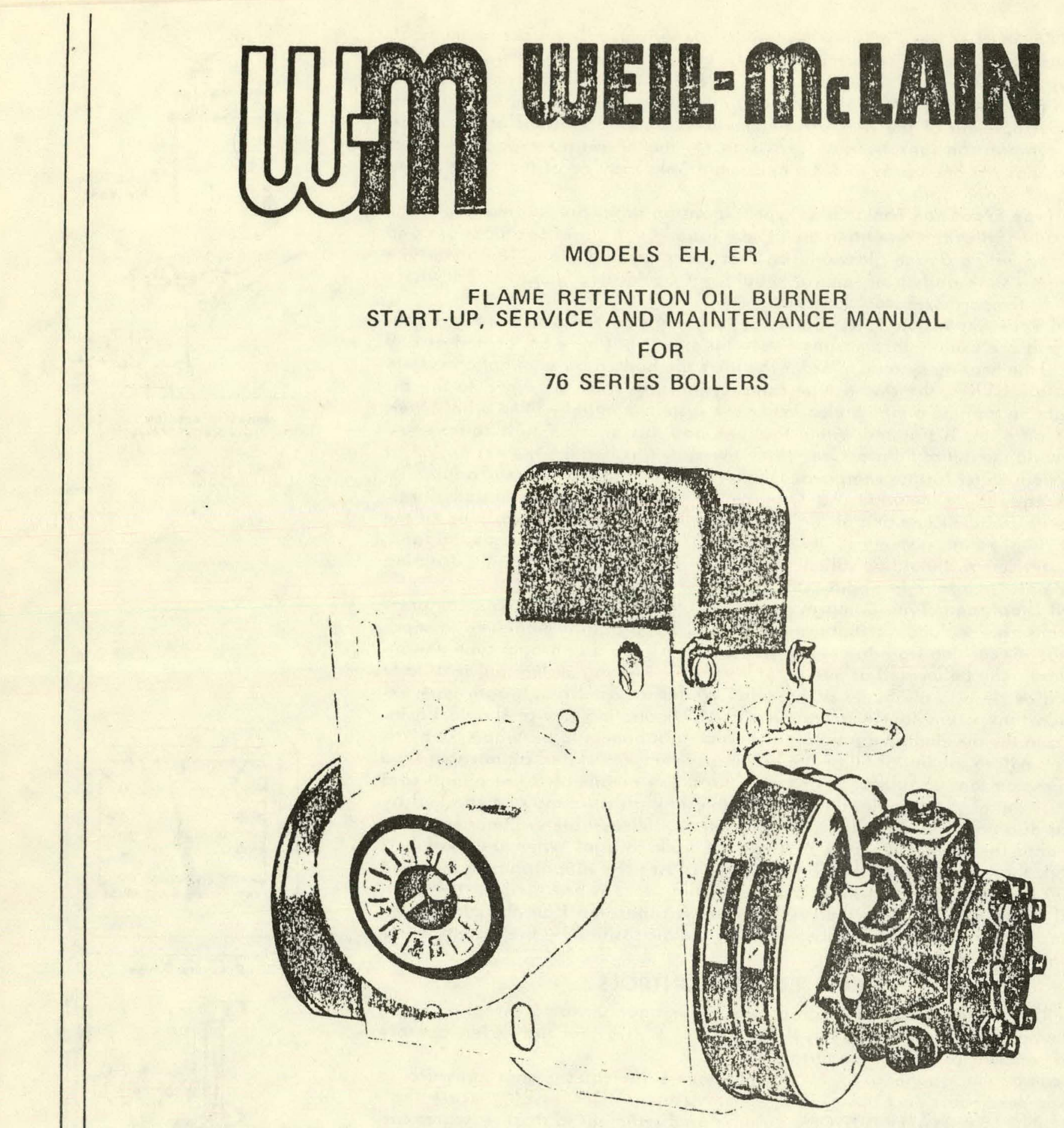

BURNER MANUFACTURED BY WAYNE HOME EQUIPMENT CO., INC.

FORT WAYNE, INDIANA

FOR

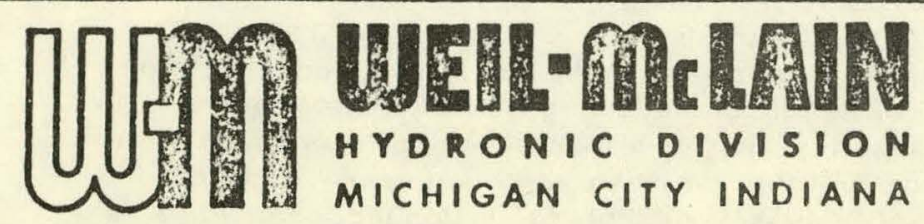




\title{
OIL BURNER CERTIFICATE
}

\author{
AS REQUUILE BY COMMERCIAL STANDARD CS75-56
}

The (Make)

Oil Burner Model No.

Serial No. installed at

(Address of Installution) bears a label evidencing compliance with commercial Standard CS75-56, and has been installed in accordance with the instructions in the manufacturer's instullation manual and in conformity. with local regulations, codes, and ordinances.

The boiler (), furnace 1 ), is a

heating loud consists of :

Btu; or

1.

3. .............. Btu, or .....

4. …......... Btu, o
2. $\ldots . . . . . . . .$. Btu, or

2. ............. Btu, or

3. ............. Btu, or take off; or

4. ............. Btu, o

4. ............. Btu, or
No. and the square feet steam ( ), hot water ( ) radiation; and

square fect of equivalent stcam ( ), hot water () radiation in domestic hot water square inches of cross-sectional area of warm air supply pipes measured at the furnace square feet of equivalent steam ( ), hot water ( ) radiation in the following special

All necessary permits have been secured, and the installation has been tested in accordance with the test procedure of Commercial Standard CS75-66 and the following readings taken:

Co. $\left\{\begin{array}{l}\text { Over Fire........ } \\ \text { At Breaching. }\end{array}\right.$

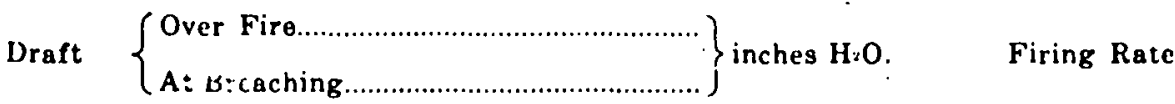
gals $\sqrt{h r}$.

All controls and limiting devices have becn checked for proper operation

Fuel used, Grade No. of Cominercial Standard CS12-48.

Field service equipment smoke scale reading

The above test results are certified to be true:

For service call:

(Name of Company making installation)

(Name)

(Address)

(Telephone)
Per

(Signature)

(Address)

(Telephone)

Date 
76 BOILER RATINGS AND DATA

\begin{tabular}{|c|c|c|c|c|c|c|c|c|c|}
\hline $\begin{array}{l}\text { BOILER } \\
\text { NUMBER }\end{array}$ & $\begin{array}{l}\text { I:B:R } \\
\text { OIL FIRING } \\
\text { RATE GPH }\end{array}$ & $\begin{array}{l}\text { GROSS } \\
\text { l:B=R } \\
\text { OUTPUT } \\
\text { BTU/Hr+ }\end{array}$ & $\begin{array}{l}\text { WATER } \\
\text { NET FB:R } \\
\text { OUTPUT } \\
\text { BTU/il: }\end{array}$ & $\begin{array}{l}\text { STEAM } \\
\text { NET I:B:R } \\
\text { OUTPUT } \\
\text { BTU/HI. }\end{array}$ & $\begin{array}{c}\text { NET } \\
\text { SOUARE } \\
\text { FEET : } \\
\text { WATER }\end{array}$ & $\begin{array}{l}\text { NET } \\
\text { SOUARE } \\
\text { FEET } \\
\text { STEAM }\end{array}$ & $\begin{array}{l}\text { DRAFT LOSS } \\
\text { THROUGH } \\
\text { BOILER.INCHES } \\
\text { WATER COLUMN }\end{array}$ & $\begin{array}{l}\text { I:B=R CH } \\
\text { SIZE } \\
\text { INCHES }\end{array}$ & $\begin{array}{l}\text { MNEY } \\
\text { EIGHT } \\
\text { FEET }\end{array}$ \\
\hline $\begin{array}{l}476 \\
576 \\
676 \\
776 \\
876 \\
976\end{array}$ & $\begin{array}{l}2.35 \\
2.95 \\
3.60 \\
4.25 \\
4.90 \\
5.55\end{array}$ & $\begin{array}{l}264,000 \\
336,000 \\
408,000 \\
480,000 \\
552,000 \\
624,000\end{array}$ & $\begin{array}{l}229,600 \\
292,200 \\
354,800 \\
417,400 \\
480,000 \\
542,600\end{array}$ & $\begin{array}{l}198,000 \\
252,100 \\
306,100 \\
360,100 \\
414,100 \\
468,100\end{array}$ & $\begin{array}{l}1530 \\
1950 \\
2365 \\
2785 \\
3200 \\
3615\end{array}$ & $\begin{array}{r}825 \\
1050 \\
1275 \\
1500 \\
1725 \\
1950\end{array}$ & $\begin{array}{l}.01 \\
.02 \\
.03 \\
.04 \\
.05 \\
.06\end{array}$ & $\begin{array}{r}8 \times 12 \\
8 \times 12 \\
12 \times 12 \\
12 \times 12 \\
12 \times 12 \\
12 \times 16\end{array}$ & $\begin{array}{l}15 \\
15 \\
17 \\
19 \\
21 \\
24\end{array}$ \\
\hline
\end{tabular}

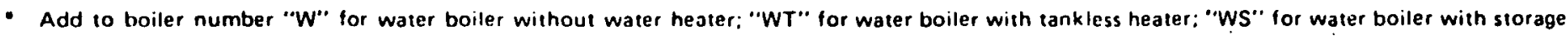
heater. "S" for steam boiler and "ST" for steam boiler with tankless heater.

- Ratings are based on $10 \% \mathrm{CO}_{2}( \pm 0.2 \%)$ in the combustion gases.

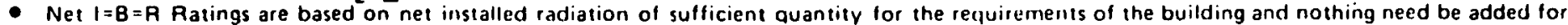

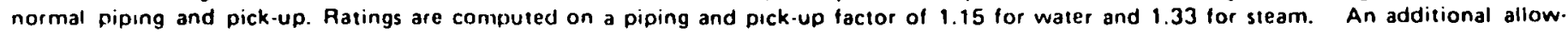
ance should be made for unusual piping and pick-up loads.

* Based on an average water temperature of $170^{\circ} \mathrm{F}$ in the Heat Distribution Units.

76 RIJRNER DATA

\begin{tabular}{|c|c|c|c|c|c|c|c|c|}
\hline $\begin{array}{c}\text { BOILER } \\
\text { MODEL } \\
\text { NO. }\end{array}$ & $\begin{array}{c}\text { BUANER } \\
\text { MODEL } \\
\text { NO. }\end{array}$ & $\begin{array}{c}\text { BURNER } \\
\text { SPEC. } \\
\text { NO. }\end{array}$ & SIZE & $\begin{array}{l}\text { NOZZLE } \\
\text { ANGLE/TYPE }\end{array}$ & $\begin{array}{l}\text { AIR } \\
\text { CONE } \\
\text { I.D. \& TYPE }\end{array}$ & $\begin{array}{c}\text { BAFFLE } \\
\text { PLATE } \\
\text { O.D. }\end{array}$ & $\begin{array}{l}\text { ELECTRODE } \\
\text { SUPPORT }\end{array}$ & $\begin{array}{l}\text { FLAMELOCK } \\
\text { SETTING, } \\
\text { Irom FACE } \\
\text { of AIR CONE }\end{array}$ \\
\hline
\end{tabular}

All of the above capacitios are based on operation at sea level, with 115/60 current, 3450 RPM motor lexcepting Spec. No. 163.39A ER Burner - 1725 RPM).

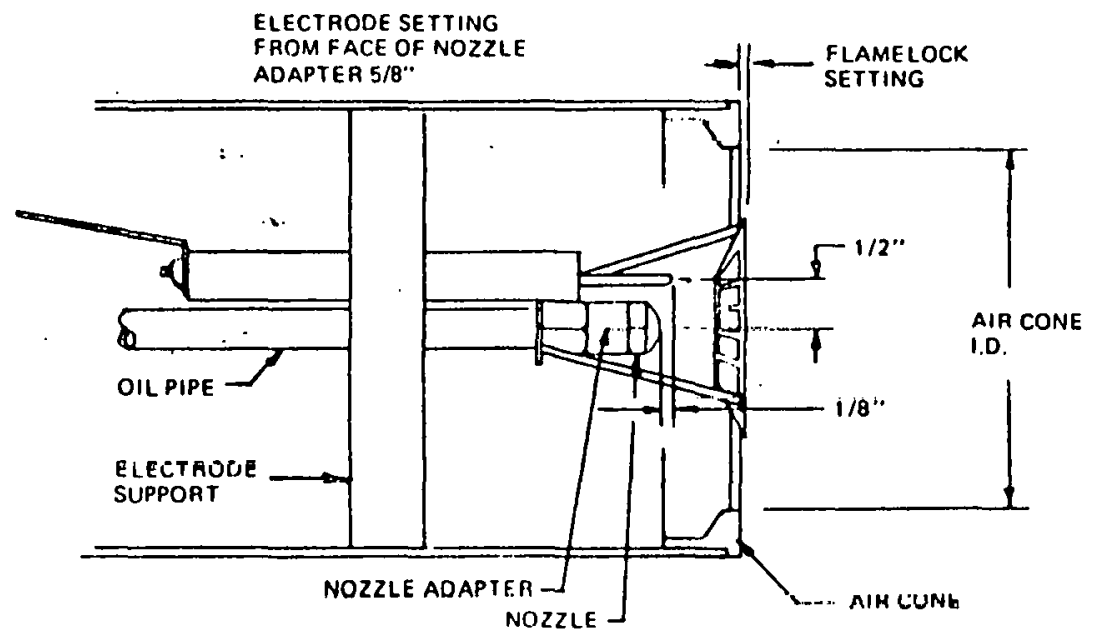

Adjustments:

The adlustments of the Flame Lock with the Air Cone could vary slightly depending upon the job conditions.

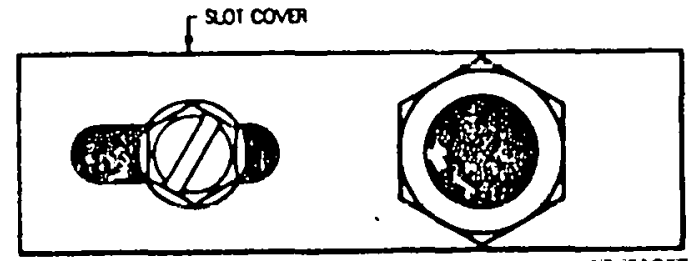

NOTE: Gun Assembly is pre set at factory. End of slot cover is set with arrow on decal as shown.

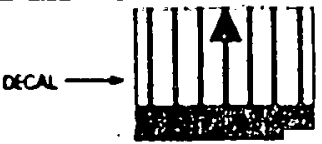

On reinstalling gun assembly. slot cover to be set as shown. 


\section{GENERAL REQUIREMENTS}

The fuel oil tank, fuel line piping, and power input wiring must be installed in accordance with the requirements of the National Board of Fire Underwriters'. Underwriters' Laboratories, and any additional national or local codes or requirements having jurisdiction. This burner is listed for use with either No. 1 or No. 2 fuel oil by the Underwriters' Laboratories, Inc., the New York Board of Standards and Appeals, the State Fire Marshall of the Commonwealth of Massachusettes, the Department of State Police in Connecticut, and others. The burner is manufactured in accordance with the National Bureau of Standards, Commercial Standard No. CS75.56.

\section{CHIMNEY REQUIREMENTS}

The natural draft chimney or vent must be at least of thc size indicated on Page 3 under the 76 Boiler Ratings and Data. Where the cross sectional area or height of the natural draft chimney or vent is smaller than the published dimensions, or where excessive resistance to the flow of combustion gases can be expected, it may be necessary to purchase and install an inexpensive induced draft fan for best operation. For elevations above 1.000 feet, the published chimney cross sectional area and height should be increased by at least four (4) per cent for each 1,000 feet above sea level.

The chimney should be examined before the connection of the boiler to be certain that it is properly constructed. clear, and will freely conduct the products of combustion to the atmosphere. The chimney or vent should extend high enough above the building or any other obstructions so that wind from any direction will not strike the chimney or vent from an angle above horizontal and thus produce down drafts. Unless the obstruction is of great magnitude, it is the usual experience that a chimney or vent extended at least two feet above flat roofs or two feet above the highest part of wall parapets and peaked roofs which are within thirty feet will be reasonably free from down drafts.

\section{BREECHING REQUIREMENTS}

In entering the chimney, the breeching connection must be sufficiently above the extreme bottom of the chimney to avoid any danger of stoppage. The breeching connection must not project beyond the inner wall of the chimney. $A$ thimble or slip joint may be used to facilitate removal of the breeching for cleaning. Do not place a damper or any other obstruction in the breeching. The breeching should slope upward toward the chimney at least $1 / 4$ inch per lineal foot of breeching and must be at least of the same equivalent diameter as the published dimensions of the rectangular or square chinney. Long horizontal breechings, excessive numbers of elbows or tees, or other obstructions which are restrictive to the flow of combustion gases should be avoided.

Be sure the barometric draft control is installed in the breeching between the boiler and chimney according to the manufacturer's instructions packaged with the control. A small hole should be drilled in the breeching just beyond the boiler smoke outlet to facilitate measuring the stack temperature, $\mathrm{CO}_{2}$, breeching draft, and for taking a smoke reading.

\section{AIR SUPPLY FOR COMBUSTION}

Provisions must be made to supply sufficient clean air to the boiler room at all times for combustion, for ventil. ation, for operation of the baromatric draft control, and to prevent less than atmospheric air pressures in the boiler room. If there is a lack of combustion air in the boiler room, the burner flame will be yellow and formation of soot will occur on the boiler flue passages. In buildings of conventional frame, brick or stone construction without enclosed utility rooms, basement storm windows, or tight stair doors, infiltration is normally adequate to provide air for combustion and for operation of the barometric draft control.

For installations in an enclosed utility room or boiler room without an outside wall, a fresh air opening to the outside with a free cross sectional area of at least twice the area of the flue outlet is recommended. For each 1,000 feet above sea level, increase the fresh air opening by at least four (4) per cent. The boiler room should be isolated from any area served by exhaust fans. Do not install an exhaust fan in the boiler room.

\section{BURNER AND SAFETY CIRCUIT WIRING}

The burner motor, ignition Iransformer, circulator and combination burner primary control, limit control, and circulator control are prewired at the factory. If any additional electrical salety contiols are employed, No. 14 gauge wire in conduit may be used. The power input supply wiring to the boiler should be No. 14 gauge or heavier wire in conduit, as required, and should have a properly sized fused disconnect switch. All wiring should be installed in accordance with the requirements of the National Electrical Code and any additional state or local code requirements having jurisdiction. Refer to the separate wiring diagram packaged in the envelope with these instructions for wiring the boiler. 
NOTICE TO INSTALLER

NEW BURNER SPEC FOR 676 BOILER

\begin{tabular}{|c|c|c|c|c|c|c|c|c|}
\hline BOILER & BURNER & BURNER & & & AIR & BAFFLE & & $\begin{array}{l}\text { FLAMELOCK } \\
\text { SETTING }\end{array}$ \\
\hline MODEL & MODEL & SPEC & & NOZZLE & CONE & PLATE & ELECTRODE & FROY EACE \\
\hline NUMEER & NUMBER & NUNBER & SIZE & ANGLE/TYFE & I.D. : TYPE & O.D. & SUPPORT & OF AIR CO:IE \\
\hline 676 & EH & $163-57$ & 3.50 & $0^{\circ}$ Solid & $3 \frac{1}{2} " 8$ Vane & None & Stabilizer (Reversed) & 3/16" Ahead \\
\hline
\end{tabular}


- TS LIST - WEIL-MCLAIN CO.

10 SERIES BOILERS

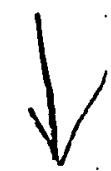

\begin{tabular}{lccccc} 
BURNER MOULL NO. & ER & EH & EH & EH & EH \\
BURNER SPEC NO. & $163-51$ & $163-53$ & $163-55$ & $163-57$ & $163-59$ \\
\hline
\end{tabular}

\section{ITEM}

1 Motor 1/8 UP $1 / 1.15 / 60 / 1725$

- Motor. 1/4 HP 1/115/60/3450

2 Screw, Motor Mounting

3 Cover, Motor Cord

4 Screw, Trans. Hold Down Clip

5 Cli.p, Trans. Hold Down

6 Screw, Fan Set (Included W/lian

7 Fan

8 Housing, Fan

9 Band, Air - Inner

10 Screw, Slot Cover

11 . Plate, Slot Cuver

12 Locknut, Oil Line

13 Line, $\mathrm{Oil}$

14 Screw, Air Band

15 Band, Air - Outer

17 Coupling E1bow

19 Pump, Fuel - Sundstrand "J"

20 . Screw, Fuel Pump Mounting

21 Fitting, 0il Pipe

22 Pipe, 0il

23 Buss Bar

24 Screw, Set (Included W/Support)

25 Screw, Rd. Head \#10-24 x 3/8"

26 Support, Flectrode - Stabtlizer

$-$

27

28

29

30

31

32

33 Screw, Air Cone \#8-32 x 5/16"

34 Bushing, Insulator

35 Pal Nut

36 Assembly, Air Tube \& Flange Assy

37 Cone, Air

45 Transformer

46 Screw, Trans. Hinge $5 / 16^{\prime \prime}-1.8 \times 1 / 2^{\prime \prime}$

- Assembly, Gun - Replacement Assembly Gun - Symbol

Assembly, Electrode - Roplacement

- Gasket, Air lube (not shown)

- Gasket, Flange (not shown)
PAR'I NO.

\begin{tabular}{|c|c|c|c|c|}
\hline 20382 & - & - & - & - \\
\hline- & 20383 & 20383 & 20383 & 20383 \\
\hline 12701 & 12701 & 1270.1 & 12701 & 12701 \\
\hline 13029 & 13029 & 13029 & 13029 & 13029 \\
\hline 13044 & 13044 & .13044 & 13044 & 13044 \\
\hline 13038 & 13038 & 13038 & 13038 & 13038 \\
\hline - & - & - & - & - \\
\hline $20289 \mathrm{~T}$ & 20288 & 20288 & $20289 \mathrm{~T}$ & $20289 \mathrm{~T}$ \\
\hline 4725 & 4725 & 4725 & 4725 & 4725 \\
\hline 2669 & 2669 & 2669 & 2669 & 2669 \\
\hline 12697 & 12697 & 1.2697 & 12697 & 12697 \\
\hline 13392 & 13392 & 13392 & 13392 & 13392 \\
\hline 14296 & $1 / 4296$ & 14296 & 14296 & 14296 \\
\hline 14452 & 14452 & 14452 & 14452 & 14452 \\
\hline 12701 & 12701 & 12701 & 12701 & 12701 \\
\hline 2668 & 2668 & 2668 & 2668 & 2668 \\
\hline 20280 & 20280 & .20280 & 20280 & 20280 \\
\hline 1.3494 & 13494 & 13494 & 13494 & 13494 \\
\hline 12336 & 13164 & 13164 & 13248 & 13248 \\
\hline 12701 & 12701 & 12701 & 12701 & 12701 \\
\hline 14295 & 14295 & 14295 & 14295 & 14295 \\
\hline 13654 & 13654 & 13654 & 13654 & 13654 \\
\hline 12458 & 12458 & 12458 & 12458 & 12458 \\
\hline- & - & - & - & - \\
\hline 1.2694 & 12694 & 12694 & 12694 & 12694 \\
\hline 14310 & - & - & 14310 & - \\
\hline- & 14308 & 14308 & 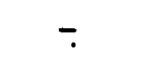 & 14308 \\
\hline None & 13408 & 13409 & None & 13407 \\
\hline 12695 & 12695 & 12695 & 12695 & 12695 \\
\hline 12354 & 12354 & 12354 & 12354 & 12354 \\
\hline 13163 & 13163 & 1.3163 & 13163 & 13163 \\
\hline 12988 & 12988 & 12988 & 12988 & 12988 \\
\hline 12903 & 12903 & 1.2903 & 12903 & 12903 \\
\hline 12699 & 12699 & 12699 & 12699 & 12699 \\
\hline 12408 & 1.2408 & 12408 & 12.408 & 12408 \\
\hline 13.110 & 13110 & 13110 & 13110 & 13110 \\
\hline 21118 & 21118 & 21118 & 21118 & 21118 \\
\hline 13702 & 12329 & 12990 & 13702 & 13008 \\
\hline 20358 & 20358 & 20358 & 20358 & 20358 \\
\hline 13045 & 13045 & 13045 & 13045 & 13045 \\
\hline 20734 & 20733 & 20733 & 20734 & 20733 \\
\hline $637 \mathrm{ER}$ & $6.37 .1 R$ & $637 \mathrm{KR}$ & $638 \mathrm{ER}$ & $637 \mathrm{LR}$ \\
\hline 13286 & 13286 & 13286 & 13286 & 13286 \\
\hline 1374.5 & 1.3745 & 1.3745 & 13745 & 13745 \\
\hline 12484 & 12484 & 12484 & 12484 & 12484 \\
\hline
\end{tabular}




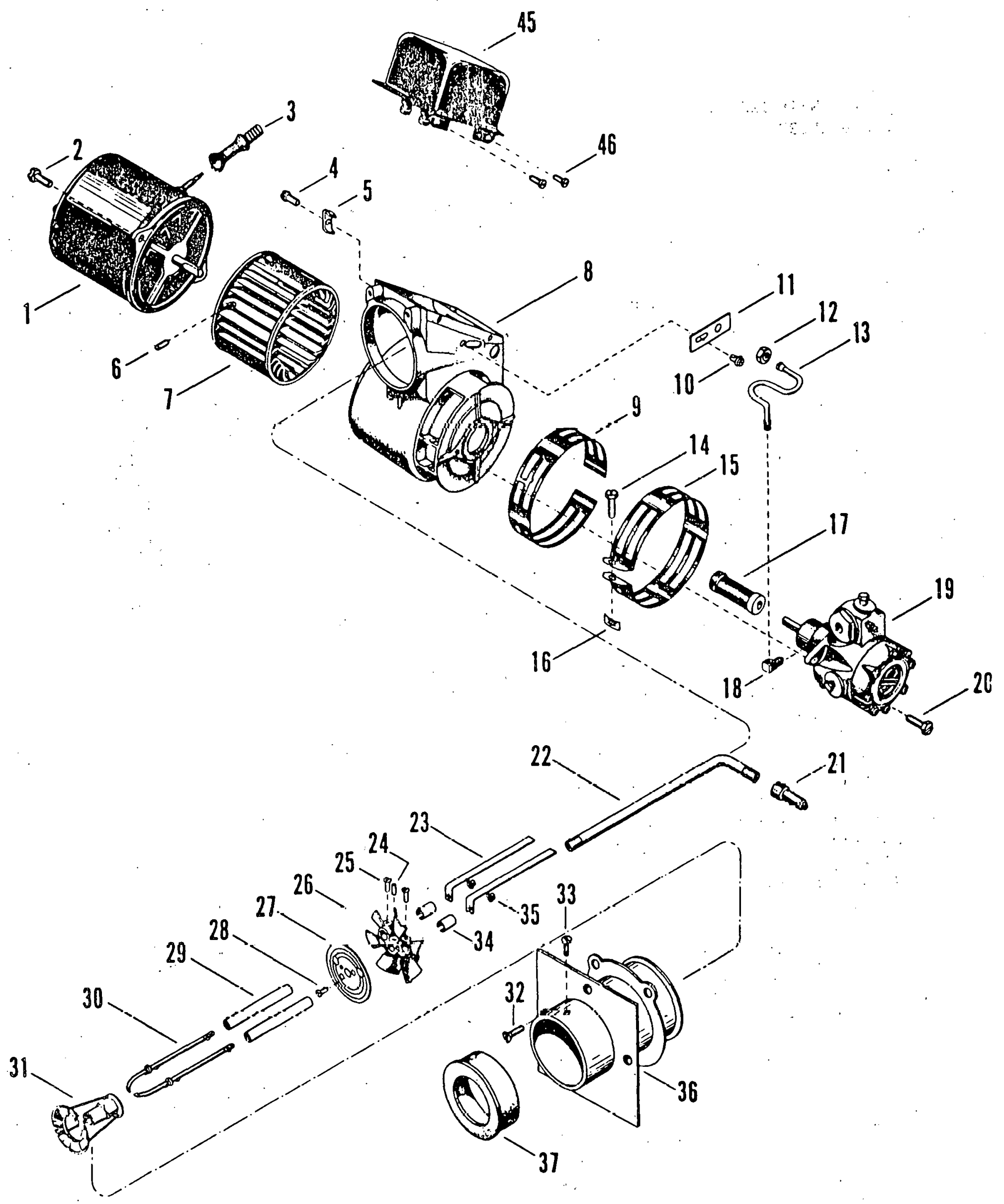


The fucl oil supplier slon:ld be consulted regarding the fuel oil storige lank capacity for the burner firing rate employed. The cules of the National Board of Fire Underwriters' and any state or local codes which may apply should be followed in locating and installing the fuel oil tank. Underwriters' Laboratories requirements stipulate a hottom outlet on all 275 gallon and larger fuel oil tanks so the lank can be drained. This is to prevent the accumulationkof condensate which causses the liank 10 iust. It is recornmended that a willer trap be installed at the tank outlet to prevent any water from entering the bunes There are a number of additives on the market that can be put into the fuel oil tank with the fucl oil; these additives hold the water in suspension and allow it to pass into the burner. Consult the local fuel oil supplier for information concerning the use of these additives.

\section{FUEL OIL SUPPLY PIPING}

A single stage fuel oil pump is furnished as standard equipment with the boiler. Refer to the fuel oil pump in. struction sheet packaged in the envelope with these instructions for additional instructions on sizing and installing the fuel oil supply piping. The rules of the National Board of Fire Underwriters' and any state or local code requirements which may apply should be followed in locating, and installing the fuel oil piping.

For all installations, it is recommended lhat an oil filter of the proper capacity be installeg in the fuel oil supply piping. Where practical, the oil filter should be located as close as possible to the oil storage tank, but not outside the building.

Copper tubing sliould he used in preference to iron pipe as it has less nossibility for leaks and does not scale off on the inside. Flare type fillings are recommended as the soldered type may melt in case of fire.

The fuel oil piping from the tank to the burner should be sized no smaller than $3 / 8$ inch O.D. copper tubing. Where the fuel oil tank is located a considerable distance from the burner, the fuel oil piping should be sized larger so that less friction loss will be encountered. Refer to fuel oil pump instruction sheet.

Connections to the Inried fuel oil storage tanks must be made with swiny joints to prevent the fuel oil piping from breaking in case the tank settles. If the job requicements stipulate that iron pipe be used, swing joints made ap with ellows and nipples seviral inches long should be employed and located as close as possible to the tank. The swing joints should be installed so they will tighten as the tank settles.

Particular calle shou!ll he exercised mut to create an air trap in the fuel oil supply niping. There is always a slight amount of air in suspension in fuel oil and if eraps are present. they will glaclually fill with air and the fuel oil pump will lose its prime. It is good piping practice to install a tee and plug.at the highest point in the fuel oil supply piping to aid in priming the fuel unit and in expelling air.

Manual shut-off oil valves should be provided in the fuel oil supply piping near the burner and at the tank or near where the fuel oil supply piping enters the building from an outside tank.

One-Pipe System: Where the funl oil storage tank is located above the burner and gravity oil flow to the burner is permissible, a one-pipe fuel oil piping system may be employed. Refer to the fuel oil pump instruction sheet for preparing the pump for a one pipe system. The fuel oil piping should be connected at the bottom of the storage tank and should slope downward loward the burner at least $1 / 2$ inch per lineal foot. The gradual slope in the fuel oil piping will help to prevent the fornation of air pockets and the collection of air bubbles in the piping which could interfere with the operation of the busuce. Where rigid iron piping is employed for the fuet oil supply piping. the supply piping should be run to a noint ditcutly bolow the burner and copper tubing should he used for connecting trom the iron pipe to the inlet port on the fucl oil pump; where copper tubing is emploved for the fuel oil supply piping. the tubing can be run directly to the inlet port on the fuel oil pump. One complete leop should be made in the copper tubing directly below the connection to the fuel oil pump; this loop will help to reduce transmission noise and prevent strain on the burner. A one-pipe fuel oil piping system is not recommended where it is necessarv to lift the oil.

Two.Pipe System: A two pipe fuel oil piping system is recornmended where it is necessary to lift the oil or where gravity flow of the oil to the burmer is not permissible. Refer to the fuel oil pump instruction sheet for preparing the pump for a two pipe system. Slip fittings should be installed at the top of the tank for both the supnly and returning pipsing. The supply and retuin piping should be run to within foul (4) to six $(6)$ inches from the hottom of the taink The reflurn niping fhruld be of the same size as the supply piping and run as directly as possible from the return opening in the fuet oil pump to the tank. The supply piping should be pilched slightly back toward the tank whenever possible: and particular care should be exercised not to create air traps in the supply piping. If any manuat shut-off oil valves are installed in the return piping, a bypass relief to the tank with an oil pressure relief valve must be provided.

\footnotetext{
Anitis $O N$

If any part of the fur! oil tank is above the level of the burnes, the sillply piping should be run to a point above the burner where an anti-siphon device must be installed to prevent the llow of oil in case of a break in the oil line.

If the lop of the fuct oil tank is bilow the level of the hurner, use a check valve in the supply piping on the burner side of the manual shul ofl oil valve nearest the tank; the check valve will prevent the flow of oil back to the tank during the bunner off period.
} 


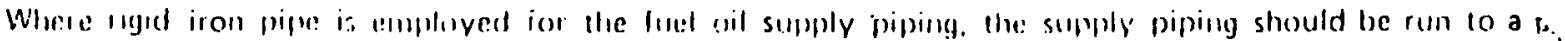

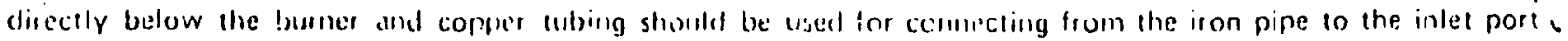

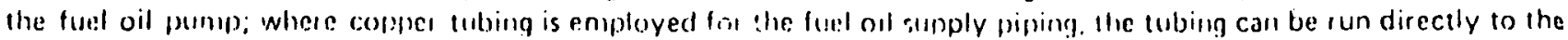

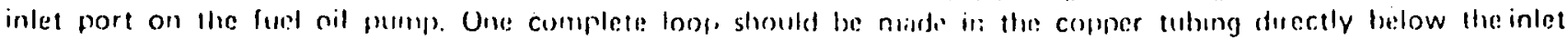
connection to the fuel oil pumb; this loop will help to reduce transmussion abise and prevent strain on the burner.

\section{FUEL OIL. PIPING TEST}

The fuel nil pipin: Jubl all fittings should he thoinughly tested for kiaks. Be sure to tighten the cover on the oil filter because the wil filter g.lsket sommtimes sluibles. Check for div loose conneclums, kinks, and possible air pockets in the fuel oul phoing. Tighton the parking nuts on any valves in.tulled in the supply piping to minimize the possibility of drawing air into the piping whule the pump is oper ating.

\section{OPERATING AND SAFETY CHECK OUT SEOUENCE}

1. Be sure the hoiler and the heat distibuting units ae filled with water to the poper pressure and that the system

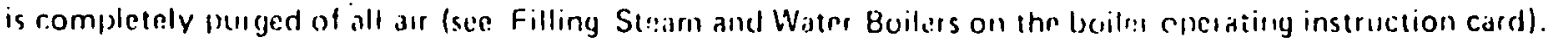

2. Be sule the main relectrice switch in the oil burnct electrical curcuit is turned th the off position.

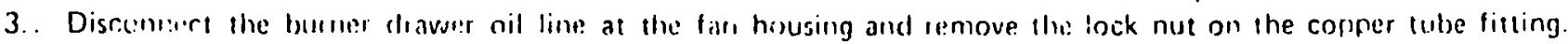

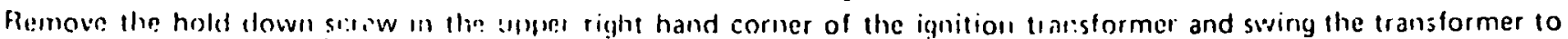

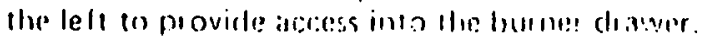

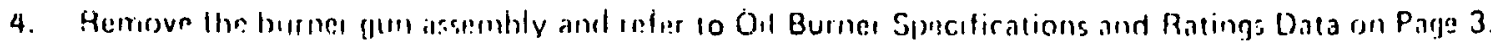

a. Chect the air handling pats and ignition electrocles to be certain they are moperly adjusted for ihe size boiler lecing installew

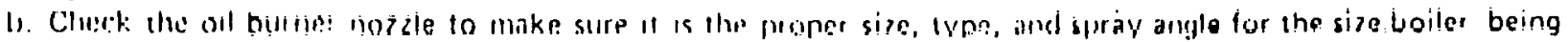
instialtecl.

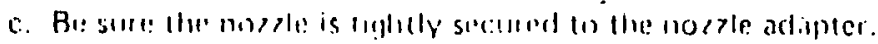

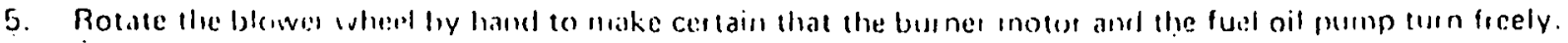

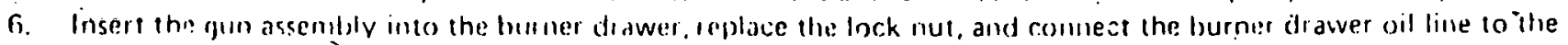
gur oil pipe. Swing the ig̣litin:s li ansformer to the right and teplace the hold down screw in the upper right hand corner.

7. Dil the bumer motor ivith ont: or two drops of good detelgent free automobile oil.

?. Busue there is sullicient fuel oil in the: storage tank to suphly the buner.

9. Make stule all nlamual oil valvens in the fue: oil piping are onen.

10. Muve the indicator on the tonin thermostat aboye the actual room iemperature so there is a call for heat.

11. Push the sutely reset lever on the burner prinaty contiol and release.

12. Tun the main electric swilch in the burner electrical circuit to the on position and the burner motor should stiart.

13. Prime the fuel oil mump by pungung all the air out of the furel line piping. If the burner primary control gocs out on safety before: all the air is conpletely purged from the fuel oil piping, it will be necessary to push the safety reset lever on the mimary sontmol in again start the burner nutor.

a. For one pipe fuel oil piping systems, mune the air out of the fuel line piping by loosening the hleed port litting on the fuel orl purmp alid wait for the oll to llow.

1). For two nipe fuel oi! pipine systems, air is automaticilly purged and diverted back to the tank hy means of the rewurn line. Loosmn the bleed port fitting on the fuel oil pump and ascertain that all the air has been' nuged fion the sur,tion tint piping.

14. When all the arr has hen elimmated from the suction line piping. furn the main electric switch in the boiler electrical circuit to the off position to ston the burner.

15. lisstall in oil pressure gaule! in the gauge put on the fuel oil punp

16. Adjust the air inlet band on the betrne to dibnut a half open position.

17. Turn the minin electries switch in the boiler electrical circuit to the on position. The burner motor should start and a humer flans: shomld be restahlishod.

18. While the burner is nperaling, check and if necessay, atjust the oil pressure. The noimal oil operating pressure sliould be 100 P.S.I.G. Tn id/lust the operating oil prassure, thin the adjusting screw clockwise to increase the operating vil pressure or cuunter reirik wise in decrease the pressure.

19. Acljust the ail inlet batul so that a cledll. yellow burner flame wilh slightlly snoky tips is established. Refrer to

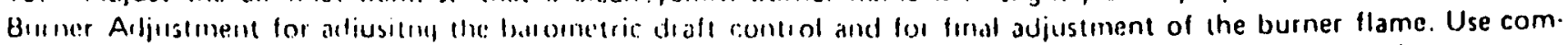

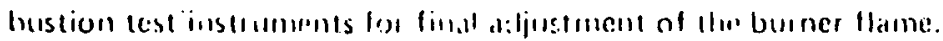

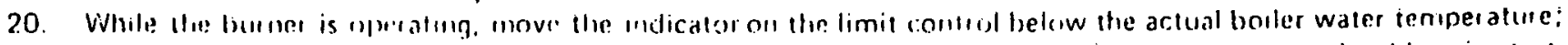
the bunee should stop. Muve the indirator on the limit zontrol to the nomal setting and the burner should aguin start.

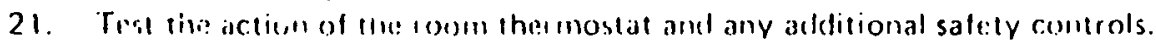

22. Stop llw: hurner and temove the nil pressure gauge from the gauge poit on the fuel oil pump and replace the ginge nort nluil.

23. Before leaving the jub, Ur sure the toom thermostat, limit control, and ally additional safety controls are properly set. 


\section{BURNER ADJUSTMENT}

After makung the necessary preliminaty burner aljustments, Iste combustion test instruments for final adjustment of the burner flame after the boiler waten temperature has be:en raisent to approxinately the design conditions. A smoke -eading of a trace to a No. 1 on the Shell Bucharach scale is recommended. Set the air intet band on the burner for the highest $\mathrm{CO}_{2}$ consistent with, a low sinoke reading.

For the initial measurements, measure the ner cent of $\mathrm{CO}_{2}$ in the rombustion gases over-fire and in the breeching. If the per cent of $\mathrm{CO}_{2}$ measured in the breeching and overfire are approximately equal, then any succeeding $\mathrm{CO}_{2}$ measurements can be made in the stack. If the per cent of $\mathrm{CO}_{2}$ measured over-fire is greater than the per cent of $\mathrm{CO}_{2}$ measure in the breeching, locate and seal any areas of the boiler that are not gas-tight.

Insert a small flame mirror into the obseivation opening in the tase front panel and view the flame front making sure the fire burns off the spinner (flamelock assenibly) approximately $1 / 8$ inch. If the fire burns on the spinner, pull the drawer assembly back; if the fire burris ahead of the spiniter by more than $1 / 8$ inch, move the gun assembly forward.

After a high $\mathrm{CO}_{2}$ and a low smoke reading have been obtained, adjust the barometric draft control to provide at least a 0.02 inches water column over-fire draft. The draft in the breeching should also be measured to determine whether there is an abnormally high dialt loss through the boiler. To obtiin the draft loss through the boiler, subtract the measured over-fire draft from the meusuted brecching draft. Compare the calculated draft with the published draft loss under Ratings and Data on Pagje 3 for the size hoiler employed. A high dlaft loss may be caused by overfiring or too much excess air and the result could be a low $\mathrm{CO}_{2}$, high smoke reading. and back piessure. lt may be necessary to again measure the per cent of $\mathrm{CO}_{2}$ and take a smoke reading after the barometric draft control has been adjusted.

After the proper combustion test results ate achieved, tighten the adjustment screw on the air inlet band to assure permanent positioning. Record the measurements obtained in the space provided on Page 2 of this manual. 


\section{DIRECTIONS FOR THE OPERATION AND CARE OF OIL BURNER}

\section{Read Instructions Carefully and Hang This Card Near Burner for Future Reference}

\section{(A) TO START BURNER:}

1. Check for oil in the storage tank.

2. Fuses in the main switch must be good.

3. Have oil butner switch open.

4. Set room thermostat about 10 degrees higher than room temperature to make sure the thermostat contacts are made. Limit control must be set high enough to make contact also.

5. Oil valve at the tank should be open and the check valve in return line properly installed so oil can return to tank.

6. Be sure nozzle of proper size for heater is in the adapter and tightly screwed down, and that the electrodes are properly spaced (See Manual). With heating plant door open, close the burner switch; and if wiring is properly done and all controls properly installed and adjusted, the burner should start. If not, check primary relay first to be sure it is properly set; and if burner does not start, recheck witing und all controls thotoughly.

7. If burner is installed with a single oil line, the fuel unit will have in be purged of the entrapped air in the oil lines and fuel unit before the oil will flow to the nozzle (See fuel unit instruction sheet for this operation). If a return line is used, purging will not be necessary, although this will speed the starting of the burner if done. If this is done, the pump should pick up its oil in less than a minute (which is the setring for the lockout switch in the primary concrol). If ignition does not take place during this time, check the nozzle and electrodes.

STARTING BURNER AFTER IGNITION FAIL.URE.

1. Do not attempt to restart butnet when excess oil has accu. mulated, when heating unit is full of vapors, or when the combustion chamber is very hot.

2. Press reset butten on primary control and burner should start. Do not attempt this more than twice. If burner fails to operate call serviceman.

(ib) FulEL OIL SPLCITICATIONG.

1. This burner is approved for oil not heavies than No. 2 The commercial standards for this oil are: Flash $110^{\circ}$ minimum or legal; Maximum $230^{\circ} \mathrm{F}$; Pour point $20^{\circ} \mathrm{F}$; Water and sediment not more than $0.1 \%$; Distillation temperature $600^{\circ} \mathrm{F}$ minimum and $675^{\circ} \mathrm{F}$ maximum at $90 \%$ of recovery. Viscosity at $100^{\circ} \mathrm{F}$ Saybolt Universal of 40 seconds maximum.

DO NOT USE GASOLINE, CRANKCASE OIL, OR ANY OIL CONTAINING GASOLINE.

(C) LUBRICATION:

1. The two oil cups on the oil burner motor should be lubricated every three months with a few drops of good grade light motor oil, No. 10 or 20 S.A.E.

(D) AT THE END OF THE HEATING SEASON:

1. Shut off electric current to burner at oil burner switch.

2. If ofl strainer has not been cleaned recently, it should be removed and cleaned (consult instructions card furnished with fuel unit).

3. Oil storage tank should be kept filled to prevent watet vapur trom collecring. It is suggested the valve in the sucrion line be closed and oil burnet switch opened. Oil scorage tank should be cleaned every 2 or 3 years to remove any sediunent or water that has collected in the tank. Your Fuel Oil Dealer has the equipment to do this.

(E) AT THE START OF THE HEATING SEASON:

1. It is advisable to have the Dealer inspect and service your burner for the coming heating season.

2. Heating plant, smoke pipe and chimney should be cleaned and checked for repairs.

3. Lubricate burner as directed under " $C$ " above.

4. It is advisahle ro have the entire electrical system inspected before pucting the burner into operation after is has been standing idle for the summer months. This sis..! include primary relay, limit control, thermostat (clean dust from contact points), and check the electrodes for carixon and cracks in insulators, and corrosion on all terminals of the eiectrodes and transformet.

(F) FMERGENCY STORS:

1. CUT OFF AILI. CURRENT TO THE BURNER BY MOV. ING 1.FVER ON THIE OIL. BURNER ELECTRIC SWITCH TO THE "OFF" POSITION.

\section{CAUTION}

1. Check the gauge in oil storage tank periodically. Keep tank filled.

2. Don's arcempr in hurn garthage or sefuse in your heacing unit.

3. Don't fill storage tank while burner is operating.

A. Don't stars burner if there is oil or vaport in the heating unit.

5. Dun't attempt to buin cranke sse drainings or crude oil.

6. DON'T TAMPER WITH BURNER OR CONTROLS CALL YOUR SERVICEMAN.

DEALER

Burner Serial No
Day Phone

Night Phone

Dace installed 


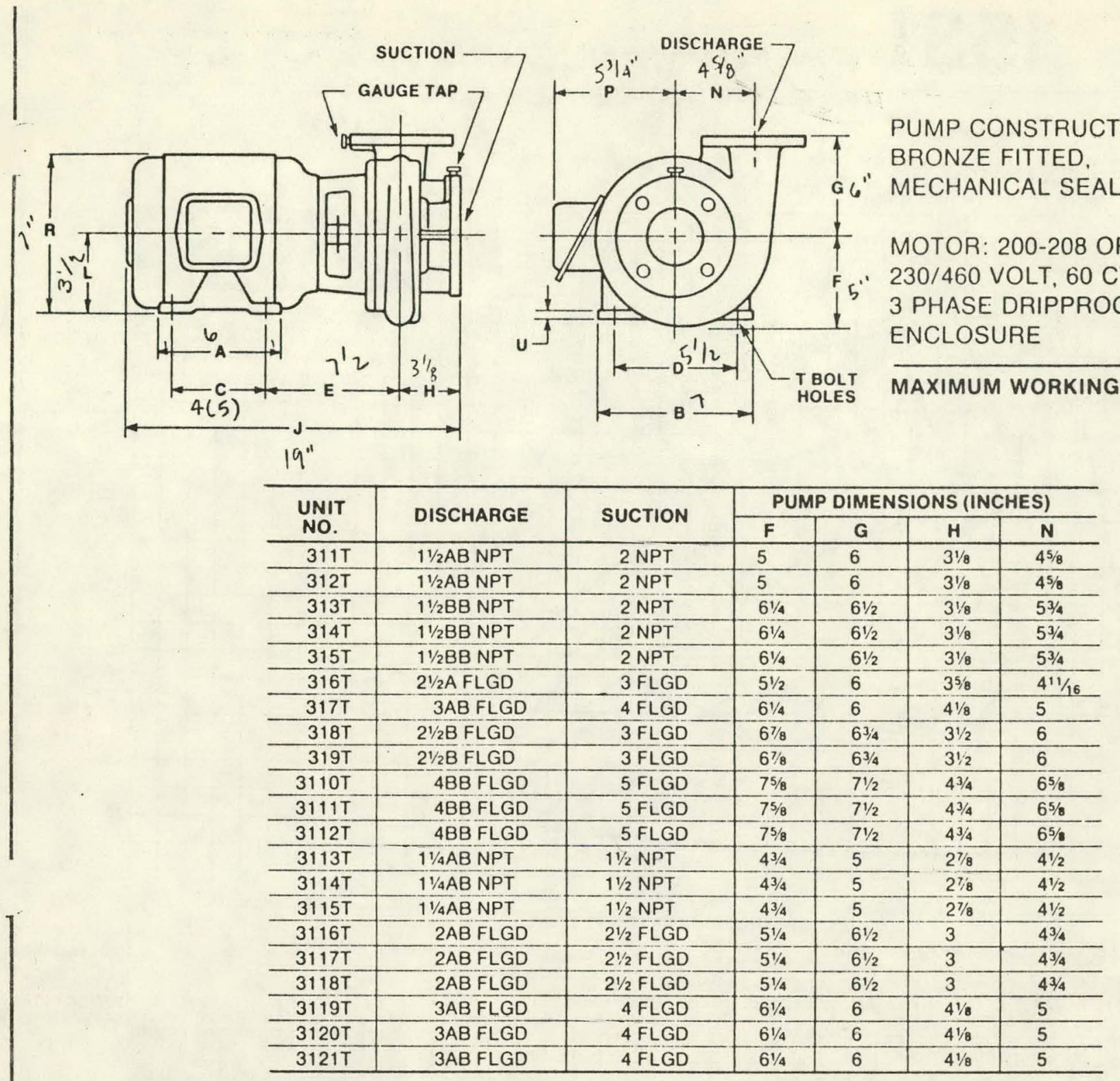

\begin{tabular}{|c|c|c|c|c|c|c|c|c|c|c|c|c|c|c|}
\hline \multirow[b]{2}{*}{$\begin{array}{l}\text { UNIT } \\
\text { NO. }\end{array}$} & \multicolumn{3}{|c|}{ MOTOR } & \multicolumn{11}{|c|}{ MOTOR DIMENSIONS (INCHES) } \\
\hline & FRAME & H.P. & R.P.M. & $\begin{array}{c}\text { A } \\
\text { (Max.) }\end{array}$ & $\begin{array}{c}\text { B } \\
\text { (Max.) }\end{array}$ & C & D & $E$ & $\begin{array}{c}J \\
\text { (Max.) }\end{array}$ & $L$ & $P$ & R & $T$ & U \\
\hline $311 \mathrm{~T}$ & 143JM & 1 & 1750 & 6 & 7 & 4 & $51 / 2$ & $71 / 2$ & $187 / 8$ & $31 / 2$ & $5^{3 / 4}$ & 7 & $11 / 32$ & $1 / 4$ \\
\hline $312 \mathrm{~T}$ & 145JM & 1.5 & 1750 & 6 & 7 & 5 & $51 / 2$ & $71 / 2$ & $18^{7 / 8}$ & $3^{1 / 2}$ & $5^{3 / 4}$ & 7 & $11 / 32$ & $1 / 4$ \\
\hline $313 \mathrm{~T}$ & $145 \mathrm{JM}$ & 2 & 1750 & 6 & 7 & 5 & $5 \frac{1 / 2}{2}$ & $71 / 2$ & $18 \%$ & $31 / 2$ & $53 / 4$ & 7 & $11 / 32$ & $1 / 4$ \\
\hline $314 \mathrm{~T}$ & $182 \mathrm{JM}$ & 3 & 1750 & $61 / 2$ & 9 & $41 / 2$ & $71 / 2$ & $81 / 4$ & $213 / 4$ & $41 / 2$ & $81 / 8$ & $91 / 4$ & $13 / 32$ & $7 / 16$ \\
\hline $315 \mathrm{~T}$ & $184 \mathrm{JM}$ & 5 & 1750 & $71 / 2$ & 9 & $51 / 2$ & $7 \frac{1 / 2}{1}$ & $81 / 4$ & $21^{3 / 4}$ & $41 / 2$ & $81 / 8$ & $91 / 4$ & $13 / 32$ & $7 / 16$ \\
\hline $316 \mathrm{~T}$ & $145 \mathrm{JM}$ & 2 & 1750 & 6 & 7 & 5 & $51 / 2$ & $79 / 16$ & $193 / 8$ & $3^{1 / 2}$ & $53 / 4$ & 7 & $11 / 32$ & $1 / 4$ \\
\hline $317 \mathrm{~T}$ & $182 \mathrm{JM}$ & 3 & 1750 & $61 / 2$ & 9 & $4 \frac{1}{2}$ & $71 / 2$ & $81 / 2$ & 23 & $41 / 2$ & $81 / 8$ & $31 / 4$ & $13 / 32$ & $7 / 16$ \\
\hline $318 \mathrm{~T}$ & 184JM & 5 & 1750 & $71 / 2$ & 9 & $51 / 2$ & $7 \frac{1}{2}$ & $83 / 8$ & $22^{1 / 4}$ & $41 / 2$ & $8^{1 / 8}$ & $9^{1 / 4}$ & $13 / 32$ & $7 / 16$ \\
\hline $319 \mathrm{~T}$ & $213 \mathrm{JM}$ & 7.5 & 1750 & $7 \frac{1}{2}$ & $101 / 2$ & $51 / 2$ & $8 \frac{1}{1 / 2}$ & $9^{1 / 4}$ & $243 / 8$ & $5^{1 / 4}$ & $83 / 4$ & $10^{3 / 4}$ & $13 / 32$ & $1 / 2$ \\
\hline $3110 \mathrm{~T}$ & $184 \mathrm{JM}$ & 5 & 1750 & $71 / 2$ & 9 & $5 \frac{1 / 2}{2}$ & $71 / 2$ & $8^{3 / 4}$ & $23^{3 / 4}$ & $4^{1 / 2}$ & $81 / 8$ & $9^{1 / 4}$ & $13 / 32$ & $7 / 16$ \\
\hline $3111 \mathrm{~T}$ & $213 \mathrm{NM}$ & $7 . \overline{5}$ & 1750 & $7 \frac{1}{2}$ & $10^{1 / 2}$ & $51 / 2$ & $8^{1 / 2}$ & $95 / 8$ & 26 & $51 / 4$ & $8^{3 / 4}$ & $10^{3 / 4}$ & $13 / 32$ & $1 / 2$ \\
\hline $3112 \mathrm{~T}$ & $215 \mathrm{JM}$ & 10 & 1750 & 9 & $101 / 2$ & 7 & $81 / 2$ & $9 \%$ & $271 / 2$ & $5 \frac{1}{1 / 4}$ & $8^{3 / 4}$ & $10^{3 / 4}$ & $13 / 32$ & $1 / 2$ \\
\hline $3113 T$ & 145JM & 3 & 3500 & 6 & 7 & 5 & $51 / 2$ & $7 \frac{1}{2}$ & $18 \frac{1}{2}$ & $3^{1 / 2}$ & $53 / 4$ & 7 & $11 / 32$ & $1 / 4$ \\
\hline $3114 \mathrm{~T}$ & $182 \mathrm{JM}$ & 5 & 3500 & $61 / 2$ & 9 & $41 / 2$ & $71 / 2$ & $81 / 4$ & $213 / 8$ & $41 / 2$ & $81 / 8$ & $9^{1 / 4}$ & $13 / 32$ & $7 / 16$ \\
\hline $3115 \mathrm{~T}$ & $184 \mathrm{JM}$ & 7.5 & 3500 & $71 / 2$ & 9 & $5^{1 / 2}$ & $71 / 2$ & $81 / 4$ & $213 / 8$ & $41 / 2$ & $81 / 8$ & $9^{1 / 4}$ & $13 / 32$ & $7 / 16$ \\
\hline $3116 \mathrm{~T}$ & $182 \mathrm{JM}$ & 5 & 3500 & $6^{1 / 2}$ & 9 & $41 / 2$ & $71 / 2$ & $81 / 4$ & $215 / 8$ & $4 \frac{1}{2}$ & $81 / 8$ & $91 / 4$ & $13 / 32$ & $7 / 18$ \\
\hline $3117 T$ & $184 \mathrm{JM}$ & 7.5 & 3500 & $71 / 2$ & 9 & $5 \frac{1}{2}$ & $71 / 2$ & $81 / 4$ & $215 \%$ & $4 \frac{1}{2}$ & $81 / 6$ & $9^{1 / 4}$ & $13 / 32$ & $7 / 18$ \\
\hline $3118 \mathrm{~T}$ & 213 JM & 10 & 3500 & $71 / 2$ & $10^{1 / 2}$ & $51 / 2$ & $8^{1 / 2}$ & $91 / 8$ & $23^{3 / 4}$ & $5^{1 / 4}$ & $83 / 4$ & $10^{3 / 4}$ & $13 / 32$ & $1 / 2$ \\
\hline $3119 \mathrm{~T}$ & $213 \mathrm{JM}$ & 10 & 3500 & $71 / 2$ & $101 / 2$ & $51 / 2$ & $81 / 2$ & $97 / 16$ & $251 / 4$ & $51 / 4$ & $8^{3 / 4}$ & $10^{3 / 4}$ & $13 / 32$ & $1 / 2$ \\
\hline $3120 \mathrm{~T}$ & $215 \mathrm{JM}$ & 15 & $3500^{-1}$ & 9 & $101 / 2$ & 7 & $81 / 2$ & $97 / 16$ & $263 / 4$ & $51 / 4$ & $83 / 4$ & $10^{3 / 4}$ & $13 / 32$ & $1 / 2$ \\
\hline $3121 \mathrm{~T}$ & 254JP & 20 & 3500 & $10^{3 / 4}$ & $12^{1 / 2}$ & $81 / 4$ & 10 & $133 / 4$ & $33^{1 / 2}$ & $6^{1 / 4}$ & $97 / 8$ & 13 & $17 / 32$ & $3 / 4$ \\
\hline
\end{tabular}




\section{PERFORMANCE CURVES}

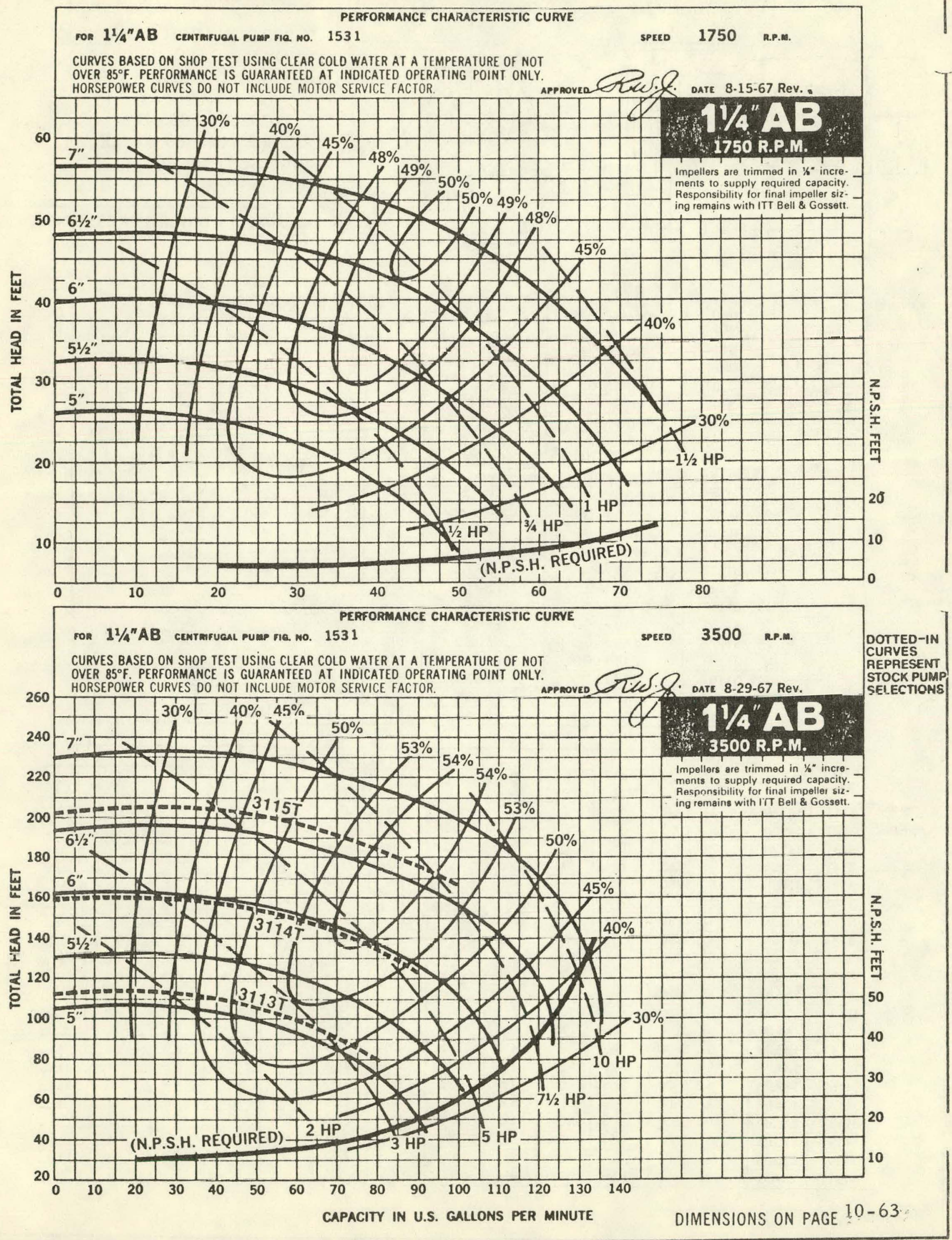


This page has been deleted because of copyright information. For information on the Series 1531 Base-mounted Centrifugal Pumps, contact International Telephone and Telegraph Corporation, Bell \& Gossett Division, Morton Grove, nlinois 60053. 